\title{
The effect of intra- and extracellular challenges on cellular responses in atherosclerosis
}

Citation for published version (APA):

van Kuijk, K. (2021). The effect of intra- and extracellular challenges on cellular responses in atherosclerosis. [Doctoral Thesis, Maastricht University]. Maastricht University. https://doi.org/10.26481/dis.20210604kk

Document status and date:

Published: 01/01/2021

DOI:

10.26481/dis.20210604kk

Document Version:

Publisher's PDF, also known as Version of record

\section{Please check the document version of this publication:}

- A submitted manuscript is the version of the article upon submission and before peer-review. There can be important differences between the submitted version and the official published version of record. People interested in the research are advised to contact the author for the final version of the publication, or visit the DOI to the publisher's website.

- The final author version and the galley proof are versions of the publication after peer review.

- The final published version features the final layout of the paper including the volume, issue and page numbers.

Link to publication

\footnotetext{
General rights rights.

- You may freely distribute the URL identifying the publication in the public portal. please follow below link for the End User Agreement:

www.umlib.nl/taverne-license

Take down policy

If you believe that this document breaches copyright please contact us at:

repository@maastrichtuniversity.nl

providing details and we will investigate your claim.
}

Copyright and moral rights for the publications made accessible in the public portal are retained by the authors and/or other copyright owners and it is a condition of accessing publications that users recognise and abide by the legal requirements associated with these

- Users may download and print one copy of any publication from the public portal for the purpose of private study or research.

- You may not further distribute the material or use it for any profit-making activity or commercial gain

If the publication is distributed under the terms of Article $25 \mathrm{fa}$ of the Dutch Copyright Act, indicated by the "Taverne" license above, 
The Effect of Intra- and Extracellular Challenges on Cellular Responses in Atherosclerosis 
(C) Kim van Kuijk, Maastricht 2021.

All rights reserved. No part of this book may be reproduced, stored in a retrieval system of any nature, or transmitted in any form or by any means (electronic, mechanical, photocopying, recording or otherwise) without prior written permission of the author, or when appropriate, by the publishers of the publications.

ISBN:

Author:

Layout \& figure design:

Cover art \& design:

Printed by:

Gesubsidieerd door
978-94-6416-477-0

Kim van Kuijk

Kim van Kuijk

Lauren Dupuis

Ridderprint | www.ridderprint.n|

Financial support by the Dutch Heart Foundation for the publication of this thesis is gratefully acknowledged.

The research described in this thesis was supported by a grant of the Dutch Heart Foundation (2016T060) 


\section{The Effect of Intra- and Extracellular Challenges on Cellular Responses in Atherosclerosis}

\section{DISSERTATION}

To obtain the degree of Doctor at Maastricht University, on the authority of Rector Magnificus, Prof. dr. Rianne M. Letschert in accordance with the decision oft he Board of Deans, tob e defended in public

On Friday, June 4th 2021, 10.00 hours

By

Kim van Kuijk 


\section{Promotors}

Prof. dr. Judith C. Sluimer

Prof. dr. Erik A.L. Biessen

\section{Co-promotor}

Prof. dr. Andrew H. Baker

\section{Assessment committee}

Prof. dr. Eline Kooi (chair)

Prof. dr. Mark Post

Prof. dr. Alma Zernecke

Dr. Helle Jørgensen

Dr. Vanessa LaPointe 


\section{Contents}

Chapter 1 General introduction and outline of the thesis

Chapter 2 Protective role of chaperone-mediated autophagy 29 against atherosclerosis

Chapter 3 Hypoxia-sensor carbonic anhydrase ix affects macrophage metabolism, but is not a suitable biomarker for human cardiovascular disease

Chapter 4 Heterogeneity and plasticity in healthy and atherosclerotic vasculature explored by single cell sequencing

Chapter 5 Deficiency of myeloid PHD proteins aggravates atherogenesis via macrophage apoptosis and paracrine fibrotic signaling

Chapter 6 Fibroblasts in atherosclerosis: heterogeneous and plastic participants

Chapter 7 Fibroblast signature genes heterogeneity revealed by single cell sequencing in murine healthy and atherosclerotic arteries

Chapter 8 General discussion

Chapter 9 Summary / Samenvatting

Chapter $10 \quad$ Impact

Appendix List of abbreviations 

Chapter 1

General introduction 



\section{Cardiovascular disease}

Cardiovascular disease (CVD) and its clinical manifestations are still the world's leading cause of death. ${ }^{1}$ In 2017, 17.9 million deaths worldwide could be attributed to cardiovascular diseases, which was an $21.1 \%$ increase compared to statistics from $2007 .^{2}$ Ischemic heart disease and stroke together accounted for roughly $85 \%$ of all cardiovascular deaths in 2017, with atherosclerosis as its main underlying cause. ${ }^{3,4}$ Risk factors for the development of CVD include smoking, blood pressure, cholesterol levels and lifestyle (diet and physical activity). ${ }^{5}$ In 2006, it was estimated that CVD would cost the European Union roughly $€ 170$ billion per year. ${ }^{6}$ This number was adjusted in 2017 to an astonishing $€ 210$ billion per year. ${ }^{7}$ Therefore targeting of CVD and its risk factors is of great interest.

\section{Healthy vasculature}

To understand how CVD is caused and how it may lead to clinical manifestations, it is important to know cell structure, plasticity and function in the healthy, steady-state vasculature. Blood vessels consist of three basic layers: tunica intima, tunica media and tunica adventitia. ${ }^{8}$ The inner tunica intima consists of a single layer of endothelial cells (ECs), which are in direct contact with circulating blood. The middle layer, the tunica media, consists mostly of smooth muscle cells (SMCs). By contracting, it regulates vascular tone and thus blood flow in the vessel. ${ }^{9}$ The current dogma is that the media is habited principally by SMCs, however, recent research using single cell sequencing (SCS) suggests that the media also harbors fibroblasts. ${ }^{10}$ However, this has neither been validated in intact tissue, nor at protein level. Adjacent to the media is the adventitial layer. This layer is comprised of extracellular matrix embedding nerves, micro-vessels, lymphatics and cells, such as macrophages, fibroblasts, and mesenchymal progenitor/stem cells. ${ }^{9}, 11$ Cells in the adventitia and medial layer closely communicate and can facilitate medial remodeling. ${ }^{12}$ This close involvement of both layers can be attributed to the progenitor/stem cells present in the adventitia. Multiple groups have shown that cells expressing stem cell markers, such as stem cell antigen-1 (Sca-1), stem cell factor receptor (c-Kit), GLI family zinc finger $r 1$ (Gli1) and CD34, reside in the adventitia and can come into action upon vascular injury. ${ }^{11,13-16}$ These findings were true both in mouse models and humans. When looking more closely at progenitor/stem cells, more overlapping features between these cells and fibroblasts can be observed. It is often shown that fibroblasts have the ability to express progenitor/stem cell markers and vice versa. Fibroblasts can express stem cell markers such as Sca-1 and cKit, encouraging the thought of fibroblasts as a progenitor cell. By using inducible tracking models, labeling c-Kit and Sca-1 positive cells, an overlap with the fibroblast marker platelet derived growth factor $\alpha$ (Pdgfr $\alpha$ ) of $20 \%$ and $40 \%$, respectively, could be observed. ${ }^{17,18}$ Moreover, gene expression analysis of healthy murine adventitia using SCS could not identify a clear progenitor/stem cell population. However, a marked increase in Sca-1 expression of a subpopulation of mesenchymal cells was shown. ${ }^{19}$ These findings indicate 


\section{1 | General Introduction}

that there might be more overlap between fibroblasts and progenitor/stem cells or that these two cell types might even be subpopulations of the same cell. In atherosclerosis, the composition of the arterial wall changes drastically and progressively and vascular cell dynamics are constantly challenged. The effect of atherosclerosis on the vasculature and its involved cell types will be discussed below.

\section{Atherosclerosis}

The development of atherosclerosis is a life-long process, characterized by EC dysfunction, lipid accumulation and inflammation, involving numerous inflammatory cells such as macrophages, T-cells and neutrophils, in the growing plaque. However, as the plaque continues to grow, SMC apoptosis takes place, leading to diminished protection from the cap and ultimately plaque rupture. ${ }^{20}$ The interplay between the involved cell types influences plaque dynamics and changes over time. The involved cell types, i.e. ECs, macrophages, SMCs and fibroblasts, will be discussed shortly below, followed by the effect of intra- and extracellular challenges on their function, plasticity and heterogeneity.

\section{Endothelial cells}

The earliest stage of atherosclerosis is characterized by endothelial dysfunction in areas prone to plaque development. Atherogenic stimuli, e.g. shear stress, blood cholesterol levels or inflammatory cytokines, can cause activation of the endothelium. ${ }^{21}$ Accumulation of the most abundant blood lipid particle, low-density lipoprotein (LDL), in the subendothelial space will stimulate endothelial dysfunction even further. These LDL particles can become oxidized (oxLDL), leading to upregulation of adhesion molecules on the endothelial cells. ${ }^{22}$ This in turn leads to attraction of circulating leukocytes and infiltration into the sub-endothelial space. The formation of a fatty streak or intimal thickening has now officially started. ${ }^{23}$ Later in plaque development, ECs contribute to newly formed intraplaque vessels. These new vessels are often malfunctioning, leading to exacerbated cytokine release and thus ingress of inflammatory cells in the surrounding plaque tissue. ${ }^{24}$ Eventually, these leaky vessels lead to intra-plaque hemorrhage, which is closely related to plaque instability and rupture. ${ }^{25}$

\section{Leukocytes}

Circulating monocytes are among the most prominent leukocyte subsets to be attracted to atherosclerotic plaque in response to stressed endothelium and inflammatory signals from the growing plaque. Upregulation of adhesion molecules, such as vascular cell adhesion molecule 1 (VCAM1) and intercellular adhesion molecule 1 (ICAM1), on the endothelial cells is triggered by the increasing plaque lipid pool and can induce firm binding of the monocytes. ${ }^{26}$ Extravasation of the monocytes into the intimal space leads to transformation into macrophages that complement the resident macrophage pool. The lipid particles 
present trigger the engulfing of oxLDL, which leads to the formation of foam cells. ${ }^{27}$ This stimulates cytokine production and thus more inflammation, leading to the beginning of a vicious circle. This stage is also known as pathological intimal thickening. ${ }^{23}$ Macrophages can be polarized into different subsets by the diversity of inflammatory cytokines present in the plaque. The classically activated (M1) and alternatively activated (M2) macrophages were always thought to be the main two subsets. The pro-inflammatory M1 macrophage can be stimulated via cytokines such as tumor necrosis factor $\alpha$ (TNF $\alpha$ ) or interferon gamma (IFN ). The anti-inflammatory M2 macrophage can be polarized via interleukin (IL)-4 and IL6. ${ }^{28,29}$ However, this dogma was challenged when transcriptomic analysis showed that polarization was not as black and white as researchers thought. ${ }^{30,31} \mathrm{~A}$ much broader range of macrophage polarization was observed, depending on the environmental stimuli present. Next to monocytes and macrophages, $T$ and $B$ cells also play prominent roles in plaque inflammation. T cells make up for roughly $30 \%$ of all leukocytes found in murine and human atherosclerosis. ${ }^{32}$ Different $T$ cell subsets have been shown to have diverse effects on atherosclerosis development.

CD4+ T helper 1 cells and natural killer T cells are thought to be pro-atherogenic, while regulatory $\mathrm{T}$ cells seem anti-atherogenic. However, CD8+ cytotoxic $\mathrm{T}$ cells, other $\mathrm{T}$ helper subset and $\gamma \delta$ T cells show controversial results in remark to atherosclerosis development. ${ }^{33}$ $B$ cells also display controversial results on atherosclerosis development, depending on subset. Unswitched, IgM producing, B cells have been shown to have a negative correlation to CVD, while activated $B$ cells correlate positively to CVD. ${ }^{34} B$ cells have the ability to produce antibodies against oxLDL, blocking the uptake of these particles and thus tempering the following immune response. ${ }^{35}$ Next to antibody production, they can also activate $T$ cells and macrophages. This can again lead to increased inflammation and associates with increased CVD risk. ${ }^{34}$ Together, abovementioned immune cells play an enormous role in the development of atherosclerosis. However, their exact role is dependent on cell type and plaque stage.

\section{Smooth muscle cells}

Smooth muscle cells (SMCs) normally reside in the medial layer beneath the newly forming plaque, as discussed above. However, during plaque progression SMCs migrate to the outer borders of the plaque forming the fibrous cap. ${ }^{36-38}$ Here, they produce extracellular matrix (ECM) to prevent plaque rupture. This stage is known as fibroatheroma. ${ }^{23}$ Switching from a quiescent, contractile state to a synthetic state enables the SMCs to produce large quantities of ECM. ${ }^{38}$ This goes hand in hand with a downregulation of SMC markers, such as $\alpha$ smooth muscle actin ( $\alpha \mathrm{SMA}$ ), smoothelin and calponin. ${ }^{39}$ The fibrous cap increases in size, protecting the plaque's content from the blood stream, forming the thick fibrous cap atheroma. ${ }^{23}$ The growing lipid pool can also trigger SMCs to undergo a transition towards macrophage-like cells, accounting for roughly $18 \%$ of CD68+ cells in human lesions. ${ }^{40}$ 


\section{1 | General Introduction}

Moreover, these macrophage-like cells are able to exert macrophage functions such as phagocytosis. ${ }^{41}$ As the plaque continues to grow with an increasing amount of foam cells, lipid core and necrotic areas, SMCs undergo apoptosis. ECM is then progressively broken down by matrix metalloproteinases (MMPs), produced by macrophages. ${ }^{37}$ These events lead to thinning of the fibrous cap, increasing plaque vulnerability. This stage is known as thin-cap atheroma. ${ }^{23}$ Ultimately the plaque can rupture, exposing its thrombogenic content to the blood stream, leading to the formation of a thrombus. Thrombi can clog vital arteries, leading to clinical manifestations such as myocardial infarction or stroke. In case of a nonocclusive thrombus, plaque development is accelerated.

\section{Fibroblasts}

Growing interest in fibroblasts in the context of atherosclerosis has been observed recently. As already discussed above, fibroblasts reside mostly in the adventitial layer of the blood vessel and contribute to processes such as ECM production and immune regulation. ${ }^{42}$ In case of vascular injury, they have been shown to migrate from the adventitia towards the media, and contribute to neointima formation and calcification. ${ }^{16,43}$ Another study showed marked proliferation of adventitial cells in case of vascular injury. ${ }^{44}$ However, these studies relied on non-specific markers as aSMA and were performed without the knowledge that we have now about fibroblasts. Fibroblasts are known to be very plastic and heterogenous, which makes current markers insufficient in identifying all fibroblast subsets. ${ }^{45}$ Moreover, fibroblasts can have various origins, e.g. SMCs, mesenchymal stem cells, ECs and myofibroblasts. The latter cells can also be a result of fibroblast (de)differentiation. Transforming growth factor $\beta$ (TGF- $\beta$ ) signaling is most known for driving myo-fibroblast differentiation. ${ }^{46}$ As myo-fibroblasts are known to produce enormous quantities of ECM, they could be of great importance in the prevention of plaque rupture. Nowadays, with the use of single cell sequencing techniques, annotation of fibroblast species in tissue has improved. ${ }^{19,47,48}$ However, multiple studies still show different numbers in terms of fibroblast presence, clusters and markers. Adventitia from atherosclerotic mice showed four clusters of fibroblasts with seemingly different functions, e.g. ECM production, immune regulation and bone formation. ${ }^{19} \mathrm{~A}$ more recent study showed two fibroblast clusters in both murine and human atherosclerosis, but also revealed a modified SMC cluster which shared the genetic landscape of both SMCs and fibroblasts. ${ }^{48}$ Together, these data imply that our current knowledge about fibroblasts shows only the tip of the iceberg and researchers need far more insight in fibroblast origin and function in atherosclerosis, before concluding about their either beneficial or detrimental effect on atherosclerosis development. 


\section{Adaptation to intra- and extracellular challenges}

As atherosclerosis is characterized by continuous environmental changes, e.g. inflammation, cellular apoptosis and lipid accumulation, cells are also continuously exposed to various environmental challenges, such as lipid overload, radical stress, and lack of oxygen. Here, different adaptations to these environmental challenges, will be discussed in relationship to cell survival, apoptosis, cell differentiation and heterogeneity among cell types involved in atherosclerosis.

\section{Autophagy and apoptosis}

Cholesterol accumulation is known to cause many cellular consequences. As the plaque grows and cholesterol from the blood accumulates in the plaque, macrophages are unable to clear these lipids. This leads to the formation of a growing lipid core. Foam cells keep taking up oxLDL particles and these oxLDL particles can trigger endoplasmic reticulum (ER) stress. Long periods of ER stress can lead to an increase in inflammation and activation of an unfolded protein response (UPR). ${ }^{49}$ UPR is the cell's homeostatic mechanism and switch between cell survival and apoptosis. When UPR is prolonged, the cell cannot maintain homeostasis and will go into apoptosis. ${ }^{50}$ Apoptosis can then be initiated via multiple pathways, of which inhibition of B-cell lymphoma-2 (Bcl-2) is one of them. ${ }^{51}$ In normal conditions, $\mathrm{Bcl}-2$ inhibits pro-apoptotic proteins such as $\mathrm{Bcl}-2$ associated $\mathrm{x}(\mathrm{Bax})$ or $\mathrm{Bcl}-2$ nineteen kilodalton interacting protein (BNIP3). In case of the latter, $\mathrm{Bcl}-2$ directly binds to BNIP3, preventing it from inducing cell death via mitochondrial reactive oxygen species (ROS) release. ${ }^{52}$ In murine atherosclerosis, it has been shown that when components of the $\mathrm{Bcl}-2$ pathway are manipulated, atherosclerosis development is altered. On the one hand, a knock-out (KO) of anti-apoptotic proteins $\mathrm{Bcl}-\mathrm{x}$ and $\mathrm{Bcl}-2$ leads to aggravated atherosclerosis through an increase of macrophage apoptosis and thus worsened plaque phenotype. ${ }^{53,54}$ However, it has also been shown that KO of pro-apoptotic proteins like p53 can lead to an increase in plaque size. ${ }^{55,56}$ This effect could be linked to the fact that macrophage apoptosis in early stages of plaque progression seems beneficial, since this suppresses plaque cellularity and therefore diminishes inflammation by plaque macrophages. ${ }^{57}$ Moreover, SMC apoptosis has been linked to decreased plaque stability because of the diminishing fibrous cap and reduced ECM production. ${ }^{58}$ Similar to macrophages, SMCs can take up oxLDL particles leading to similar apoptotic pathways as described above..$^{58,59}$

Cells can also omit the initiation of apoptosis, by undergoing autophagy. Autophagy can be seen as the recycling process of internal cellular components and therefore is, like apoptosis, also seen as a damage control process. ${ }^{60}$ Autophagy is known to exist in three forms: macroautophagy, microautophagy and chaperone-mediated autophagy (CMA). The latter will be the main form discussed in this thesis. In contrast to macroautophagy, CMA is 


\section{1 | General Introduction}

a selective procedure, where proteins with a unique targeting motif in their amino acid sequence (KFERQ sequence) are selected for lysosomal degradation. ${ }^{61}$ Once recognized by a chaperone in the cytosol, heat shock cognate 70 (HSC70), the protein is transferred to the lysosome, where it enters the lysosome via lysosome-associated membrane protein type 2A (LAMP2A) ${ }^{61}$ As this lysosomal receptor is only involved in CMA, it can be used as a marker for the activity of this type of autophagy. In the liver, it has been shown that defective CMA dysregulates liver metabolism and causes hepatosteatosis. ${ }^{62}$ Interestingly, protein homeostasis was unchanged in young mice. Compensation by other forms of autophagy can take place, assuring normal protein homeostasis. However, during aging this compensation becomes defective and hepatic homeostasis is disrupted. This in turn leads to higher susceptibility to stressors, e.g. oxidative stress. ${ }^{63}$ Moreover, CMA can be altered in response to different atherogenic stimuli linked to atherosclerosis, e.g. oxidative stress or lipid levels. ${ }^{61,64}$ In case of the latter, it has been shown that prolonged exposure to high lipid levels can lead to decreased CMA activity as lipid composition of the lysosomal membrane is altered. This leads to increased LAMP2A degradation and thus altered CMA activity. ${ }^{64}$ This mechanism has also been observed to play a role in declined CMA activity with aging. Defective plaque CMA could possibly influence atherosclerosis development, as all abovementioned factors play a role in plaque progression. However, very little is known about CMA in atherosclerosis and whether it is beneficial or detrimental could possibly depend on the cell type that is performing CMA and plaque stage. ${ }^{65}$

\section{Hypoxic signaling}

As discussed above, progressive hypoxia represents another challenge to vascular cells in the atherosclerotic plaque, Nutrient and oxygen flow to the growing core is limited, leading to persistent oxygen deficit in the plaque. Low oxygen levels, also known as hypoxia, have been shown to be present in both human and murine atherosclerosis. ${ }^{66,67}$ In human atherosclerosis, hypoxic plaque areas could be visualized by pimonidazole, which was injected before carotid endarterectomy was performed. ${ }^{66}$ This agent forms adducts in viable, but hypoxic cells $\left(\mathrm{pO}_{2} \leq 10 \mathrm{~mm} \mathrm{Hg}\right)$ and can later be detected ex vivo using immunohistochemical techniques. ${ }^{68}$

Hypoxia is regulated via hypoxia inducible factors (HIF), which are comprised of an oxygendependent $\alpha$ subunit, and a continuously expressed $\beta$ subunit. Moreover, there are three HIF $\alpha$ forms (HIF1 $\alpha$-HIF $2 \alpha-H I F 3 \alpha$ ), of which HIF1 $\alpha$ and HIF $2 \alpha$ are most studied. ${ }^{69}$ HIF1 $\alpha$ and HIF $2 \alpha$ share their domain structure and are widely expressed, while HIF3 $\alpha$ seems structurally different and its precise function remains to be elucidated. ${ }^{70,71}$ Under normal oxygen conditions, the HIF $\alpha$ subunit is hydroxylated by prolyl hydroxylase domain proteins (PHDs). The hydroxylated HIFs are recognized by an E3 ubiquitin ligase, von Hippel-Lindau protein $(\mathrm{pVHL})$, and proteasomal degradation takes place accordingly. ${ }^{72}$ There are three PHD isoforms, PHD1, -2 , and -3 (also named EgIn2, -1 , and -3 respectively), which all have 
the need for oxygen for proper functioning in common. The PHD enzymes are members of the Fe(II) and 2-oxoglutaratedependent dioxygenase family. ${ }^{73}$ When hypoxia occurs, the PHD enzymes are no longer functional, leading to stabilization of the HIF $\alpha$ subunit in the cytoplasm. The HIF $\alpha$ subunit then translocates to the nucleus, where it forms a dimer with the HIF $\beta$ subunit. ${ }^{74}$ The dimer then transcriptionally regulates expression of hypoxia regulated genes via hypoxia response elements (HRE), amongst others vascular endothelial growth factor (VEGF), Glucose transporter 1 (GLUT1), erythropoietin (EPO) and BNIP3. These HREs relate to numerous genes involved in cellular processes, such as metabolism, apoptosis and inflammation. ${ }^{74}$ In atherosclerosis, PHD1 deficiency showed a protective phenotype, linked to decreased cholesterol levels through enhanced cholesterol excretion via the feces. ${ }^{75}$ For PHD3, it has been shown that overexpression of this isoform in atherosclerosis accelerated plaque progression. ${ }^{76}$ Of the three isoforms, PHD2 is most studied. Its role in atherogenesis has been studied via genetically and pharmaceutically models. A complete KO model is however unfeasible as this is embryonically lethal. ${ }^{77}$ Pharmaceutical inhibition by using a general PHD inhibitor protected against atherosclerosis in low density lipoprotein receptor (LDLR) KO mice. Moreover, a decrease in hepatic cholesterol metabolism was observed, accompanied by an increase in oxLDL autoantibodies in the blood stream. ${ }^{78}$ To investigate whether this pharmaceutical inhibition could be attributed to PHD2 inhibition specifically, an hypomorphic mouse with reduced PHD2 mRNA was crossed to the atheroprone mouse model LDLR ${ }^{\mathrm{HLB301}} .{ }^{78} \mathrm{~A}$ similar reduction in plaque size could be observed in the hypomorphic mouse model, however, the marked decrease in serum cholesterol could not be replicated. As the inhibitor in question inhibits all PHD isoforms, this effect could also be caused by PHD1 inhibition as this isoform is linked to increased cholesterol clearance. ${ }^{75}$ As the model did show increased auto-antibodies against oxLDL, the observed athero-protective effect of the model is attributed to this phenomenon. Other disease models, e.g. hind limb ischemia and tumor development, have shown that haplodeficiency of PHD2 led to increased angiogenesis and thus increased blood flow. ${ }^{79,80}$ This impacts ischemic reperfusion and the ability of chemotherapy agents to reach the growing tumor.

In relation to atherosclerosis, systemic effects have already been described to a certain extent as stated above, while cell type specific effects are not yet clear. Specifically, the impact of PHD isoform KO in myeloid cells has not been investigated yet. This is of great importance, as myeloid cells, or more specifically macrophages, are most susceptible to hypoxia in the atherosclerotic plaques because of their high turnover of metabolic substrates. Diminished oxygen levels alter their ability to clear cholesterol and apoptotic cells, causing an increase in necrotic core and thus decreased plaque stability. ${ }^{81}$

As all three isoforms impact HIF signaling and thus regulation HRE responsive genes, PHD inhibitors have been designed to stimulate expression of such genes. Anemia, a common 


\section{1 | General Introduction}

consequence of chronic kidney disease (CKD), is one of the diseases of which the PHD inhibitors could be of great use. Anemia is characterized by decreased red blood cell number in the blood and decreased EPO production. ${ }^{82}$ PHD inhibition could lead to increased EPO production, therefore alleviating disease burden in CKD patients. Multiple PHD inhibitors, selective for PHD2, have already enrolled in clinical trials, of which Roxadustat is already approved for treatment in China. ${ }^{83}$ As CKD patients often show increased risk scores for CVD, these inhibitors could also affect cardiovascular outcome. ${ }^{84}, 85$ Hence it is of importance to have full knowledge of the function of PHDs in CVD.

Where PHD enzymes control HIF signaling in normoxic conditions, there are multiple downstream effectors that reflect HIF $\alpha$ stabilization and thus activity. One of these indicators is carbonic anhydrase IX (CAIX), a pH regulator which is mostly known from the cancer field. ${ }^{86,87}$ Under normal conditions, CAIX is only expressed in stomach and gall bladder. However, it is upregulated in numerous cancers where its upregulation is linked to poor prognosis. ${ }^{88-90}$ The CAIX gene has an HRE in its promotor, with HIF1 as its main transcriptional driver. ${ }^{87}$ By upregulating CAIX via HIF1, cancer cells promote their survival by neutralizing the acidic tumor environment. CAIX is not only a transmembrane protein, but can, after shedding by metalloproteases, also be secreted (sCAIX). ${ }^{91}$ sCAIX plasma levels have been useful in predicting prognosis in cancer patients. ${ }^{92-94}$ Moreover, in cancer cells it has been shown that CAIX expression protected against apoptosis. ${ }^{95}$ Interestingly, CAIX expression and function have not been studied in atherosclerosis. In atherosclerosis, hypoxia and an acidic environment have been linked to a more proatherogenic and proinflammatory plaque phenotype. ${ }^{96}$ To be more precise, low $\mathrm{pH}$ in macrophages has been shown to lead to $\mathrm{M} 1$ polarization and disrupted lipid handling. ${ }^{96,97}$ As hypoxia and low $\mathrm{pH}$ are prominent aspects of atherosclerosis, CAIX and its soluble form sCAIX could be interesting in predicting plaque status and clinical event prognosis, without using costly, or invasive measures.

\section{Cell plasticity and heterogeneity}

All abovementioned processes can have an influence on cell presentation, shape and function. Environmental factors can cause a cell to undergo plastic, reversible changes into a cell subtype needed at that moment in time. Growing evidence concerning mesenchymal heterogeneity and plasticity will shortly be discussed here:

Mesenchymal cell is a very broad cell annotation covering, among others, SMCs, pericytes and fibroblasts. Especially the latter cell type has been shown to be very plastic in numerous organs. ${ }^{98,99}$ This plasticity may seem to derive from the fact that fibroblasts have a plethora of possible origins and stem-cell properties, which will be discussed below.

Endothelial-to-mesenchymal transition (EndMT): This process was already described early in embryonic development, where epithelial cells transformed into a mesoderm to from the 
primitive cardiac tube (EMT). Later, endothelial cells undergo EndMT to form the atrioventricular septum. ${ }^{100,101}$ In the adult cardiovascular system, cardiac fibrosis can be a result of EndMT. ${ }^{102}$ Moreover, EndMT is also observed in several other pathologies, including murine and human atherosclerotic plaque development. Here, a fairly large proportion of fibroblast-like cells (4-18\%) was proposed to be from endothelial origin. ${ }^{103}$ In EndMT, endothelial cells loose endothelial markers on both transcriptional, and translational level, while gaining mesenchymal markers, such as fibroblast activation protein (FAP), alpha smooth muscle actin ( $\alpha \mathrm{SMA}$ ) and Transgelin (Tgln). ${ }^{103}$ EndMT can be triggered by multiple stimuli, including hypoxia, TGF- $\beta$ signaling, shear stress, cellular metabolism, epigenetic regulation or non-coding RNAs. ${ }^{100,103}$ EndMT has been linked to decreased plaque stability, as there is an upregulation of MMPs and thus increased ECM breakdown.

Modified SMCs: This recently discovered subset of SMCs can, genetically, be viewed as a hybrid between classical SMCs and fibroblasts, and is also known as fibromyocytes. ${ }^{48}$ It can be found both in murine and human atherosclerosis and is characterized by high $\mathrm{T}$ Cell factor (Tcf)-21 expression. Tcf21 is involved in cardiac development and downregulation of Tcf21 leads to an increase of classical SMC genes. Important to note is that fibromyocytes are only present in atherosclerosis and expand upon prolonged exposure to a cholesterolrich diet. Moreover, high Tcf21 expression was inversely correlated to the risk of developing coronary artery disease, thus implying that fibromyocytes may have an athero-protective role. $^{48}$

Stem cell differentiation: Research into multiple vascular injury models, e.g. calcification and atherosclerosis, has shown that progenitor cells have a substantial influence on disease development. Stem/progenitor cells have already been described briefly in the section concerning healthy vasculature, but will be more extensively described in diseased vasculature here. Stem cells are characterized by markers, such as Sca-1, Gli-1, c-Kit and CD34, of which Sca-1 has been mostly studied in context of atherosclerosis. Single cell sequencing of Sca-1+ cells of both normal healthy mice and atherosclerosis-prone apolipoprotein E (ApoE) -/- mice revealed that Sca-1+ cells in the latter mice group show a more migratory phenotype, which could be linked to lipid-loading of these cells. ${ }^{104}$ Moreover, upon atherosclerosis development, these Sca-1+ cells can migrate towards the newly formed intima and contribute to the SMC population. ${ }^{13}$ This process is, however, not a one-way street and differentiated SMCs can also contribute to the stem/progenitor pool via induction of Krüppel like factor 4 (KLF4), also involved in SMC-to-macrophage differentiation. ${ }^{105}$ Another population of Gli-1+ cells, originating from the adventitia, also migrated towards the intima in ApoE-/- mice and contributed heavily to calcification in this area. ${ }^{16}$ It can be discussed if these populations are one and the same. Sca-1+ cells are not only limited to differentiation into vascular cells, but can also differentiate into immune 


\section{1 | General Introduction}

cells. ${ }^{106}$ In atherosclerosis, this Sca-1+CD45+ cell subset increased in population size and mostly resided in the adventitia. Moreover, Sca-1+CD45+ cells did not only lead to formation of macrophages, but also contributed to the CD31+ endothelial cell pool. Transfer of Sca-1+CD45+ cells into ApoE-/- mice led to an increase in CD31+ cells and facilitated the growth of new vasa vasorum and thus blood flow. ${ }^{107}$

A key technique in further annotating these plastic cells is single cell sequencing (SCS). SCS could give more insight in fundamental points including the transcriptional differences between stem cells (Sca-1+/Gli-1+/c-Kit+) and fibroblasts, as characteristic lines are blurry between these two cell types. SCS was already used in lung, where it was able to annotate six transcriptionally different fibroblast clusters, which increased to seven upon lung fibrosis. ${ }^{47}$ These were linked to various functional profiles, e.g. matrix production, myofibroblasts and a new subset involved in lipid handling, the lipofibroblast. Moreover, in murine heart SCS was able to annotate 11 different fibroblast clusters. Upon myocardial infarction, the dynamics and presence of these clusters was altered, depending on the period after the infarction. ${ }^{108}$ In vasculature, where fibroblasts are mostly present in the tunica adventitia, there is only one study conducted using SCS. ${ }^{19} \mathrm{~A}$ limitation of this study is the amount of sequenced mesenchymal cells, therefore only giving superficial insight. To put the numbers in perspective, beforementioned study only used 840 cells for analysis. Including more cells would increase transcriptional depth and give more insight in vascular fibroblast heterogeneity. As specific fibroblast markers are scarce, better and more accurate markers are needed for correct annotation. Again, SCS could aid in this correct annotation in the vasculature. It must be warranted that markers from other organs, e.g. lung or kidney, may not suffice in annotating fibroblasts in the vasculature.

In summary, atherosclerosis is a life-long process with numerous cell types influencing its development. Heterogeneity and plasticity are two key concepts in understanding cell-tocell variation and pathways in atherosclerosis development. Intra- and extracellular challenges can ultimately trigger cellular adaptation, and intervention in these processes could influence plaque development and thus clinical outcome. 


\section{Aim and outline thesis}

In this thesis, I aimed to investigate intra- and extracellular challenges in the developing atherosclerotic plaque and its relationship to macrophage and fibroblast functioning, both involved in plaque progression. I will discuss the influence of plaque hypoxia, cell survival mechanisms and the extent of mesenchymal cell plasticity \& heterogeneity in murine and human atherosclerosis. Therefore, I hypothesized that:

1. Chaperone mediated autophagy (CMA) is protective in atherosclerosis. (Chapter 2)

2. Carbonic anhydrase IX (CAIX) and its soluble form sCAIX are biomarkers for cardiovascular disease. (Chapter 3)

3. Myeloid hypoxia handling via prolyl hydroxylase domain proteins (PHDs) influences atherosclerosis in an isoform-dependent manner. (Chapter 5)

4. Healthy murine adventitia harbors a heterogenous population of mesenchymal cells (Chapter 7)

\section{Outline thesis}

Atherosclerosis is characterized by lipid accumulation, foam cell formation, apoptosis, ultimately forming a necrotic core. In case of extracellular stress, cells can choose to degrade intracellular compartments, a process known as autophagy. A subtype of autophagy, chaperone-mediated autophagy (CMA), is a characterized by targeted degradation of proteins by a specific motif through the lysosomal membrane protein LAMP2A. ${ }^{61}$ In chapter 2, we made use of murine LAMP2A KO and knock-in (KI) models on LDLR KO background to assess atherosclerosis development.

CAIX is a marker for hypoxia and is, together with its soluble form SCAIX, widely used in cancer research and estimation of patient prognosis. ${ }^{87-90}$ In chapter 3, we investigate whether (s)CAIX could function as a biomarker in cardiovascular disease. Moreover, we examined the role of CAIX in macrophages, as these cells are known to be very susceptible to hypoxia in atherosclerosis.

In chapter 4, we will go more into detail about how SCS attributed to cell annotation, including immune, SMC, EC and fibroblast subsets, in the vasculature. Heterogeneity and plasticity are two key concepts in understanding cell biology and cellular adaption in healthy and atherosclerotic vasculature. SCS has been an helpful tool in exploring these two concepts.

Continuing with macrophages, we analyzed the effect of myeloid deletion of the three PHD isoform in LDLR KO mice in chapter 5. The PHD enzymes are the main regulators of hypoxia by HIF $\alpha$ ubiquitination and thus degradation. ${ }^{72}$ Effects of PHD deletion was examined in terms of in vitro cellular responses, in vivo plaque development and plaque composition. 


\section{1 | General Introduction}

Chapter 6 discusses in depth the role of fibroblasts in healthy and atherosclerotic vasculature and how their role in the vasculature became more prominently valued over the last years.

The adventitia has been shown to be a progenitor niche for the vasculature, including multiple immune cells, fibroblasts and resident vascular progenitor cells. ${ }^{11}$ In chapter 7 , we explore the genetic landscape of cells positive for mesenchymal marker platelet derived growth factor $\beta$ (PDGFR $\beta$ ) in healthy murine adventitia, by using single cell sequencing (SCS).

In chapter 8, we will discuss the main findings of this thesis in a general discussion. 


\section{References}

1. Roth GA, Johnson C, Abajobir A, et al. Global, Regional, and National Burden of Cardiovascular Diseases for 10 Causes, 1990 to 2015. J Am Coll Cardiol. 2017;70:1-25.

2. Collaborators GBDCoD. Global, regional, and national age-sex-specific mortality for 282 causes of death in 195 countries and territories, 1980-2017: a systematic analysis for the Global Burden of Disease Study 2017. Lancet. 2018;392:1736-1788.

3. Joseph $\mathrm{P}$, Leong $\mathrm{D}, \mathrm{McKee} \mathrm{M}$, et al. Reducing the Global Burden of Cardiovascular Disease, Part 1: The Epidemiology and Risk Factors. Circ Res. 2017;121:677-694.

4. Herrington W, Lacey B, Sherliker P, et al. Epidemiology of Atherosclerosis and the Potential to Reduce the Global Burden of Atherothrombotic Disease. Circ Res. 2016;118:535-46.

5. Timmis A, Townsend N, Gale C, et al. European Society of Cardiology: Cardiovascular Disease Statistics 2017. Eur Heart J. 2018;39:508-579.

6. Leal J, Luengo-Fernandez R, Gray A, et al. Economic burden of cardiovascular diseases in the enlarged European Union. Eur Heart J. 2006;27:1610-9.

7. Wilkins $E$ WL, Wickramasinghe $K$, Bhatnagar $P$, Leal J, Luengo-Fernandez R, Burns R, Rayner M, Townsend N. European Cardiovascular Disease Statistics 2017. European Heart Network, Brussels. 2017.

8. Tucker WD and Mahajan K. Anatomy, Blood Vessels StatPearls Treasure Island (FL); 2020.

9. Mazurek R, Dave JM, Chandran RR, et al. Vascular Cells in Blood Vessel Wall Development and Disease. Adv Pharmacol. 2017;78:323-350.

10. Kalluri AS, Vellarikkal SK, Edelman ER, et al. Single-Cell Analysis of the Normal Mouse Aorta Reveals Functionally Distinct Endothelial Cell Populations. Circulation. 2019;140:147-163.

11. Majesky MW, Dong XR, Hoglund V, et al. The adventitia: a progenitor cell niche for the vessel wall. Cells Tissues Organs. 2012;195:73-81.

12. Di Wang H, Ratsep MT, Chapman A, et al. Adventitial fibroblasts in vascular structure and function: the role of oxidative stress and beyond. Can J Physiol Pharmacol. 2010;88:177-86.

13. Hu Y, Zhang Z, Torsney E, et al. Abundant progenitor cells in the adventitia contribute to atherosclerosis of vein grafts in ApoE-deficient mice. J Clin Invest. 2004;113:1258-65.

14. Pasquinelli G, Tazzari PL, Vaselli C, et al. Thoracic aortas from multiorgan donors are suitable for obtaining resident angiogenic mesenchymal stromal cells. Stem Cells. 2007; 25:1627-34.

15. Passman JN, Dong XR, Wu SP, et al. A sonic hedgehog signaling domain in the arterial adventitia supports resident Sca1+ smooth muscle progenitor cells. Proc Natl Acad Sci U S A. 2008;105:9349-54. 


\section{1 | General Introduction}

16. Kramann R, Goettsch C, Wongboonsin J, et al. Adventitial MSC-like Cells Are Progenitors of Vascular Smooth Muscle Cells and Drive Vascular Calcification in Chronic Kidney Disease. Cell Stem Cell. 2016;19:628-642.

17. Ni Z, Deng J, Potter CMF, et al. Recipient c-Kit Lineage Cells Repopulate Smooth Muscle Cells of Transplant Arteriosclerosis in Mouse Models. Circ Res. 2019;125:223-241.

18. Tang J, Wang H, Huang X, et al. Arterial Sca1(+) Vascular Stem Cells Generate De Novo Smooth Muscle for Artery Repair and Regeneration. Cell Stem Cell. 2020;26:81-96 e4. 19. Gu W, Ni Z, Tan YQ, et al. Adventitial Cell Atlas of wt (Wild Type) and ApoE (Apolipoprotein E)-Deficient Mice Defined by Single-Cell RNA Sequencing. Arterioscler Thromb Vasc Biol. 2019;39:1055-1071.

20. Falk E. Pathogenesis of atherosclerosis. J Am Coll Cardiol. 2006;47:C7-12.

21. Gimbrone MA, Jr. and Garcia-Cardena G. Endothelial Cell Dysfunction and the Pathobiology of Atherosclerosis. Circ Res. 2016;118:620-36.

22. Kita T, Kume N, Minami M, et al. Role of oxidized LDL in atherosclerosis. Ann N Y Acad Sci. 2001;947:199-205; discussion 205-6.

23. Sakakura K, Nakano M, Otsuka F, et al. Pathophysiology of atherosclerosis plaque progression. Heart Lung Circ. 2013;22:399-411.

24. Sedding DG, Boyle EC, Demandt JAF, et al. Vasa Vasorum Angiogenesis: Key Player in the Initiation and Progression of Atherosclerosis and Potential Target for the Treatment of Cardiovascular Disease. Front Immunol. 2018;9:706.

25. Michel JB, Virmani R, Arbustini E, et al. Intraplaque haemorrhages as the trigger of plaque vulnerability. Eur Heart J. 2011;32:1977-85, 1985a, 1985b, 1985c.

26. Moore KJ, Sheedy FJ and Fisher EA. Macrophages in atherosclerosis: a dynamic balance. Nat Rev Immunol. 2013;13:709-21.

27. Webb NR and Moore KJ. Macrophage-derived foam cells in atherosclerosis: lessons from murine models and implications for therapy. Curr Drug Targets. 2007;8:1249-63.

28. Mantovani A, Garlanda C and Locati M. Macrophage diversity and polarization in atherosclerosis: a question of balance. Arterioscler Thromb Vasc Biol. 2009;29:1419-23.

29. Wilson HM. Macrophages heterogeneity in atherosclerosis - implications for therapy. J Cell Mol Med. 2010;14:2055-65.

30. Xue J, Schmidt SV, Sander J, et al. Transcriptome-based network analysis reveals a spectrum model of human macrophage activation. Immunity. 2014;40:274-88.

31. Stremmel C, Stark K and Schulz C. Heterogeneity of Macrophages in Atherosclerosis. Thromb Haemost. 2019;119:1237-1246.

32. Winkels $\mathrm{H}$, Ehinger $\mathrm{E}$, Vassallo $\mathrm{M}$, et al. Atlas of the Immune Cell Repertoire in Mouse Atherosclerosis Defined by Single-Cell RNA-Sequencing and Mass Cytometry. Circ Res. 2018;122:1675-1688.

33. Saigusa R, Winkels $\mathrm{H}$ and Ley K. T cell subsets and functions in atherosclerosis. Nat Rev Cardiol. 2020. 
34. Sage AP, Tsiantoulas D, Binder CJ, et al. The role of B cells in atherosclerosis. Nat Rev Cardiol. 2019;16:180-196.

35. Kearney JF. Immune recognition of OxLDL in atherosclerosis. J Clin Invest. 2000;105:1683-5.

36. Basatemur GL, Jorgensen $\mathrm{HF}$, Clarke $\mathrm{MCH}$, et al. Vascular smooth muscle cells in atherosclerosis. Nat Rev Cardiol. 2019;16:727-744.

37. Bennett MR, Sinha S and Owens GK. Vascular Smooth Muscle Cells in Atherosclerosis. Circ Res. 2016;118:692-702.

38. Doran AC, Meller N and McNamara CA. Role of smooth muscle cells in the initiation and early progression of atherosclerosis. Arterioscler Thromb Vasc Biol. 2008;28:812-9.

39. Beamish JA, He P, Kottke-Marchant K, et al. Molecular regulation of contractile smooth muscle cell phenotype: implications for vascular tissue engineering. Tissue Eng Part B Rev. 2010;16:467-91.

40. Shankman LS, Gomez D, Cherepanova OA, et al. KLF4-dependent phenotypic modulation of smooth muscle cells has a key role in atherosclerotic plaque pathogenesis. Nat Med. 2015;21:628-37.

41. Rong JX, Shapiro M, Trogan E, et al. Transdifferentiation of mouse aortic smooth muscle cells to a macrophage-like state after cholesterol loading. Proc Natl Acad Sci U SA. 2003;100:13531-6.

42. Kuwabara JT and Tallquist MD. Tracking Adventitial Fibroblast Contribution to Disease: A Review of Current Methods to Identify Resident Fibroblasts. Arterioscler Thromb Vasc Biol. 2017;37:1598-1607.

43. Li G, Chen SJ, Oparil S, et al. Direct in vivo evidence demonstrating neointimal migration of adventitial fibroblasts after balloon injury of rat carotid arteries. Circulation. 2000;101:1362-5.

44. Shi Y, O'Brien JE, Fard A, et al. Adventitial myofibroblasts contribute to neointimal formation in injured porcine coronary arteries. Circulation. 1996;94:1655-64.

45. Tillie RJH VKK, Sluimer JC. Fibroblasts in atherosclerosis. Curr opin Lipidol (submitted). 2020.

46. Singh S and Torzewski M. Fibroblasts and Their Pathological Functions in the Fibrosis of Aortic Valve Sclerosis and Atherosclerosis. Biomolecules. 2019;9.

47. Xie T, Wang Y, Deng N, et al. Single-Cell Deconvolution of Fibroblast Heterogeneity in Mouse Pulmonary Fibrosis. Cell Rep. 2018;22:3625-3640.

48. Wirka RC, Wagh D, Paik DT, et al. Atheroprotective roles of smooth muscle cell phenotypic modulation and the TCF21 disease gene as revealed by single-cell analysis. Nat Med. 2019;25:1280-1289.

49. Andres V, Pello OM and Silvestre-Roig C. Macrophage proliferation and apoptosis in atherosclerosis. Curr Opin Lipidol. 2012;23:429-38. 


\section{1 | General Introduction}

50. Tabas I. Macrophage apoptosis in atherosclerosis: consequences on plaque progression and the role of endoplasmic reticulum stress. Antioxid Redox Signal. 2009;11:2333-9.

51. Linton MF, Babaev VR, Huang J, et al. Macrophage Apoptosis and Efferocytosis in the Pathogenesis of Atherosclerosis. Circ J. 2016;80:2259-2268.

52. Burton TR and Gibson SB. The role of Bcl-2 family member BNIP3 in cell death and disease: NIPping at the heels of cell death. Cell Death Differ. 2009;16:515-23.

53. Shearn Al, Deswaerte V, Gautier EL, et al. Bcl-x inactivation in macrophages accelerates progression of advanced atherosclerotic lesions in Apoe(-/-) mice. Arterioscler Thromb Vasc Biol. 2012;32:1142-9.

54. Thorp E, Li Y, Bao L, et al. Brief report: increased apoptosis in advanced atherosclerotic lesions of Apoe-/- mice lacking macrophage $\mathrm{Bcl}-2$. Arterioscler Thromb Vasc Biol. 2009;29:169-72.

55. Liu J, Thewke DP, Su YR, et al. Reduced macrophage apoptosis is associated with accelerated atherosclerosis in low-density lipoprotein receptor-null mice. Arterioscler Thromb Vasc Biol. 2005;25:174-9.

56. van Vlijmen BJ, Gerritsen G, Franken AL, et al. Macrophage p53 deficiency leads to enhanced atherosclerosis in APOE*3-Leiden transgenic mice. Circ Res. 2001;88:780-6.

57. Seimon T and Tabas I. Mechanisms and consequences of macrophage apoptosis in atherosclerosis. J Lipid Res. 2009;50 Suppl:S382-7.

58. Clarke MC, Figg N, Maguire JJ, et al. Apoptosis of vascular smooth muscle cells induces features of plaque vulnerability in atherosclerosis. Nat Med. 2006;12:1075-80.

59. Grootaert MOJ, Moulis M, Roth L, et al. Vascular smooth muscle cell death, autophagy and senescence in atherosclerosis. Cardiovasc Res. 2018;114:622-634.

60. Martinet W, Coornaert I, Puylaert P, et al. Macrophage Death as a Pharmacological Target in Atherosclerosis. Front Pharmacol. 2019;10:306.

61. Kaushik $\mathrm{S}$ and Cuervo AM. The coming of age of chaperone-mediated autophagy. Nat Rev Mol Cell Biol. 2018;19:365-381.

62. Schneider JL, Suh Y and Cuervo AM. Deficient chaperone-mediated autophagy in liver leads to metabolic dysregulation. Cell Metab. 2014;20:417-32.

63. Schneider JL, Villarroya J, Diaz-Carretero A, et al. Loss of hepatic chaperonemediated autophagy accelerates proteostasis failure in aging. Aging Cell. 2015;14:249-64.

64. Rodriguez-Navarro JA, Kaushik S, Koga H, et al. Inhibitory effect of dietary lipids on chaperone-mediated autophagy. Proc Natl Acad Sci U S A. 2012;109:E705-14.

65. Hassanpour M, Rahbarghazi R, Nouri M, et al. Role of autophagy in atherosclerosis: foe or friend? J Inflamm (Lond). 2019;16:8.

66. Sluimer JC, Gasc JM, van Wanroij JL, et al. Hypoxia, hypoxia-inducible transcription factor, and macrophages in human atherosclerotic plaques are correlated with intraplaque angiogenesis. J Am Coll Cardiol. 2008;51:1258-65. 
67. Parathath S, Mick SL, Feig JE, et al. Hypoxia is present in murine atherosclerotic plaques and has multiple adverse effects on macrophage lipid metabolism. Circ Res. 2011;109:1141-52.

68. Krohn KA, Link JM and Mason RP. Molecular imaging of hypoxia. J Nucl Med. 2008;49 Suppl 2:129S-48S.

69. Schofield CJ and Ratcliffe PJ. Oxygen sensing by HIF hydroxylases. Nat Rev Mol Cell Biol. 2004;5:343-54.

70. Webb JD, Coleman ML and Pugh CW. Hypoxia, hypoxia-inducible factors (HIF), HIF hydroxylases and oxygen sensing. Cell Mol Life Sci. 2009;66:3539-54.

71. Yang SL, Wu C, Xiong ZF, et al. Progress on hypoxia-inducible factor-3: Its structure, gene regulation and biological function (Review). Mol Med Rep. 2015;12:2411-6.

72. Lee JW, Ko J, Ju C, et al. Hypoxia signaling in human diseases and therapeutic targets. Exp Mol Med. 2019;51:1-13.

73. Jaakkola P, Mole DR, Tian YM, et al. Targeting of HIF-alpha to the von Hippel-Lindau ubiquitylation complex by O2-regulated prolyl hydroxylation. Science. 2001;292:468-72.

74. Majmundar AJ, Wong WJ and Simon MC. Hypoxia-inducible factors and the response to hypoxic stress. Mol Cell. 2010;40:294-309.

75. Marsch E, Demandt JA, Theelen TL, et al. Deficiency of the oxygen sensor prolyl hydroxylase 1 attenuates hypercholesterolaemia, atherosclerosis, and hyperglycaemia. Eur Heart J. 2016;37:2993-2997.

76. Liu H, Xia Y, Li B, et al. Prolyl hydroxylase 3 overexpression accelerates the progression of atherosclerosis in ApoE-/- mice. Biochem Biophys Res Commun. 2016;473:99-106.

77. Takeda K, Ho VC, Takeda H, et al. Placental but not heart defects are associated with elevated hypoxia-inducible factor alpha levels in mice lacking prolyl hydroxylase domain protein 2. Mol Cell Biol. 2006;26:8336-46.

78. Rahtu-Korpela L, Maatta J, Dimova EY, et al. Hypoxia-Inducible Factor Prolyl 4Hydroxylase-2 Inhibition Protects Against Development of Atherosclerosis. Arterioscler Thromb Vasc Biol. 2016;36:608-17.

79. Mazzone $M$, Dettori $D$, de Oliveira RL, et al. Heterozygous deficiency of PHD2 restores tumor oxygenation and inhibits metastasis via endothelial normalization. Cell. 2009;136:839-851.

80. Takeda Y, Costa S, Delamarre E, et al. Macrophage skewing by Phd2 haplodeficiency prevents ischaemia by inducing arteriogenesis. Nature. 2011;479:122-6.

81. Marsch E, Theelen TL, Demandt JA, et al. Reversal of hypoxia in murine atherosclerosis prevents necrotic core expansion by enhancing efferocytosis. Arterioscler Thromb Vasc Biol. 2014;34:2545-53.

82. Joharapurkar AA, Pandya VB, Patel VJ, et al. Prolyl Hydroxylase Inhibitors: A Breakthrough in the Therapy of Anemia Associated with Chronic Diseases. J Med Chem. 2018;61:6964-6982. 


\section{1 | General Introduction}

83. Dhillon S. Roxadustat: First Global Approval. Drugs. 2019;79:563-572.

84. Chen N, Hao C, Peng X, et al. Roxadustat for Anemia in Patients with Kidney Disease Not Receiving Dialysis. N Engl J Med. 2019;381:1001-1010.

85. Chen N, Hao C, Liu BC, et al. Roxadustat Treatment for Anemia in Patients Undergoing Long-Term Dialysis. N Engl J Med. 2019;381:1011-1022.

86. Ames S, Andring JT, McKenna R, et al. CAIX forms a transport metabolon with monocarboxylate transporters in human breast cancer cells. Oncogene. 2020;39:17101723.

87. Pastorek J and Pastorekova S. Hypoxia-induced carbonic anhydrase IX as a target for cancer therapy: from biology to clinical use. Semin Cancer Biol. 2015;31:52-64.

88. Kaya AO, Gunel N, Benekli M, et al. Hypoxia inducible factor-1 alpha and carbonic anhydrase IX overexpression are associated with poor survival in breast cancer patients. J BUON. 2012;17:663-8.

89. Sowa T, Menju T, Chen-Yoshikawa TF, et al. Hypoxia-inducible factor 1 promotes chemoresistance of lung cancer by inducing carbonic anhydrase IX expression. Cancer Med. 2017;6:288-297.

90. Ambrosio MR, Di Serio C, Danza G, et al. Carbonic anhydrase IX is a marker of hypoxia and correlates with higher Gleason scores and ISUP grading in prostate cancer. Diagn Pathol. 2016;11:45.

91. Zatovicova M, Sedlakova O, Svastova E, et al. Ectodomain shedding of the hypoxiainduced carbonic anhydrase IX is a metalloprotease-dependent process regulated by TACE/ADAM17. Br J Cancer. 2005;93:1267-76.

92. HyrsI L, Zavada J, Zavadova Z, et al. Soluble form of carbonic anhydrase IX (CAIX) in transitional cell carcinoma of urinary tract. Neoplasma. 2009;56:298-302.

93. Zavada J, Zavadova Z, Zat'ovicova $M$, et al. Soluble form of carbonic anhydrase IX (CA IX) in the serum and urine of renal carcinoma patients. Br J Cancer. 2003;89:1067-71.

94. Ilie M, Mazure NM, Hofman V, et al. High levels of carbonic anhydrase IX in tumour tissue and plasma are biomarkers of poor prognostic in patients with non-small cell lung cancer. Br J Cancer. 2010;102:1627-35.

95. Vidlickova I, Dequiedt F, Jelenska L, et al. Apoptosis-induced ectodomain shedding of hypoxia-regulated carbonic anhydrase IX from tumor cells: a double-edged response to chemotherapy. BMC Cancer. 2016;16:239.

96. Oorni K, Rajamaki K, Nguyen SD, et al. Acidification of the intimal fluid: the perfect storm for atherogenesis. J Lipid Res. 2015;56:203-14.

97. Park SY and Kim IS. Identification of macrophage genes responsive to extracellular acidification. Inflamm Res. 2013;62:399-406.

98. Sato $\mathrm{Y}$ and Yanagita $\mathrm{M}$. Functional heterogeneity of resident fibroblasts in the kidney. Proc Jpn Acad Ser B Phys Biol Sci. 2019;95:468-478.

99. Lighthouse JK and Small EM. Transcriptional control of cardiac fibroblast plasticity. J Mol Cell Cardiol. 2016;91:52-60. 
100. Kovacic JC, Dimmeler S, Harvey RP, et al. Endothelial to Mesenchymal Transition in Cardiovascular Disease: JACC State-of-the-Art Review. J Am Coll Cardiol. 2019;73:190-209. 101. Kovacic JC, Mercader N, Torres M, et al. Epithelial-to-mesenchymal and endothelial-to-mesenchymal transition: from cardiovascular development to disease. Circulation. 2012;125:1795-808.

102. Zeisberg EM, Tarnavski O, Zeisberg M, et al. Endothelial-to-mesenchymal transition contributes to cardiac fibrosis. Nat Med. 2007;13:952-61.

103. Evrard SM, Lecce L, Michelis KC, et al. Endothelial to mesenchymal transition is common in atherosclerotic lesions and is associated with plaque instability. Nat Commun. 2016;7:11853.

104. Kokkinopoulos I, Wong MM, Potter CMF, et al. Adventitial SCA-1(+) Progenitor Cell Gene Sequencing Reveals the Mechanisms of Cell Migration in Response to Hyperlipidemia. Stem Cell Reports. 2017;9:681-696.

105. Majesky MW, Horita H, Ostriker A, et al. Differentiated Smooth Muscle Cells Generate a Subpopulation of Resident Vascular Progenitor Cells in the Adventitia Regulated by Klf4. Circ Res. 2017;120:296-311.

106. Psaltis PJ, Puranik AS, Spoon DB, et al. Characterization of a resident population of adventitial macrophage progenitor cells in postnatal vasculature. Circ Res. 2014;115:36475.

107. Toledo-Flores D, Williamson A, Schwarz N, et al. Vasculogenic properties of adventitial Sca-1(+)CD45(+) progenitor cells in mice: a potential source of vasa vasorum in atherosclerosis. Sci Rep. 2019;9:7286.

108. Farbehi N, Patrick R, Dorison A, et al. Single-cell expression profiling reveals dynamic flux of cardiac stromal, vascular and immune cells in health and injury. Elife. $2019 ; 8$. 



\section{Chapter 2}

\section{Protective role of chaperone-mediated autophagy against atherosclerosis}

Madrigal-Matute J, de Bruijn J, van Kuijk K, Tiano S, Diaz A, Gijbels M, Sander B, Tasset I, Biessen EAL, Martín-Segura A, Bourdenx M, Jin H, Rensen P, Berbée J, Pasterkamp G, Sluimer JC*, Cuervo AM*

*Shared authorship

In preparation 



\section{Abstract}

Chaperone-mediated autophagy (CMA) contributes to regulation of energy homeostasis by timely degradation of enzymes involved in glucose and lipid metabolism. Here, we investigated the role of CMA in murine and human atherosclerosis. A murine atherosclerosis model, lacking CMA rate-limiting protein lysosome associated membrane protein 2A (LAMP2A), showed aggravated plaque development accompanied by altered blood lipid profiles. The latter was linked to a change in overall metabolism, characterized by increased body weight, glucose intolerance and insulin resistance. LAMP2A ko vascular smooth muscle cells engulfed more lipids, leading to an increase in genes linked to dedifferentiation of smooth muscle cells and cell death. In immune cells, LAMP2A ko led to skewing towards a more pro-inflammatory state, as shown by nitric oxide signaling. Human atherosclerotic plaques showed an increase in LAMP2A protein expression along plaque progression, presumably as a protective mechanism. In human plaque lysates, we surprisingly found that decreased LAMP2A protein expression was linked to development of a secondary cardiac event. As CMA is altered by dietary lipids, this could be an important factor in maintaining a balance in CMA activity. Lastly, we used a mouse model with induced CMA via incorporation of the human LAMP2A gene in their genome. Here, we were able to show that upregulation of CMA was atheroprotective and decreased plaque vulnerability. Moreover, blood lipid levels showed a complete opposite phenotype compared to LAMP2A KO mice. We propose that CMA could be an attractive therapeutic target against cardiovascular diseases. 


\section{2 | CMA in atherosclerosis}

\section{Introduction}

Cardiovascular disease (CVD) is the leading underlying cause of death worldwide accounting for more than $31.5 \%$ of total deaths ${ }^{1}$. The main risk factors for the development of atherosclerosis - the most common cause of CV clinical events - such as obesity, hypertension, diabetes and aging are rising in epidemic proportions due to changes in lifestyle and the growing elderly population ${ }^{1}$. In atherosclerosis, hypercholesterolemia leads to vascular endothelial dysfunction and extravasation of atherogenic lipoproteins, resulting in increased adhesion and extravasation of monocytes from the circulation to the intima ${ }^{2,3}$. Once there, monocytes engulf modified low-density lipoproteins (LDL), differentiate into macrophages and foam cells and proliferate forming a neointima with a lipid-laden macrophage core ${ }^{4}, 5$. High cytokine secretion and production of nitric oxide (NO) and reactive oxygen species (ROS) at the lipid-, necrotic-, and macrophage-rich regions creates a pro-inflammatory and oxidative environment that drives dedifferentiation of vascular smooth muscle cells (SMC) from a contractile to an activated secretory and migratory phenotype ${ }^{6,7,89}$. Activated SMC migrate from the media into the intima, further increasing inflammation, oxidative stress and collagen and elastin deposition at the fibrous cap ${ }^{2}$. The pro-inflammatory, oxidative and hypoxic environment in the plaque exacerbates cellular toxicity and cell death and promotes vascular calcification and matrix degradation ${ }^{10,11}$, which together make the plaque prone to rupture and often result in the subsequent clinical event $^{12}$.

Autophagy mediates the degradation of cellular components in lysosomes, thus assuring intracellular quality control and cellular energetics through recycling of essential catabolites $^{13}$. Chaperone-mediated autophagy (CMA) is a selective type of autophagy for proteins bearing a pentapeptide motif (KFERQ-like motif) ${ }^{14,15}$. The heat shock cognate protein (HSC70) recognizes this motif and delivers substrate proteins to lysosomeassociated membrane protein type 2A (LAMP-2A), that serves as receptor and translocation complex upon multimerization ${ }^{16,17}$. Substrate proteins reach the lysosomal lumen through this complex assisted by the lysosomal-resident $\mathrm{HSC}^{18-20}$. Besides removal of oxidized and damaged proteins by $\mathrm{CMA}$, selective and timely degradation of fully functional proteins by this type of autophagy terminates their function. This regulated remodeling of the proteome by CMA is behind its participation in the regulation of multiple intracellular processes, such as glucose and lipid metabolism, cell cycle, transcriptional programs or T-cell activation, among others ${ }^{21,22,23}$. In fact, in vivo blockage of CMA in liver results in exacerbated glycolysis and lipogenesis ${ }^{21}$ and blocks lipolysis ${ }^{22}$. Although basal levels of CMA are detectable in most mammalian cells, CMA is upregulated in response to proteotoxicity ${ }^{24}$, lipotoxicity ${ }^{25}$, oxidative stress $^{26}$ and hypoxia ${ }^{27}$, all conditions that contribute to the etiology of atherosclerosis ${ }^{2}$. Reduced CMA activity - due to lower stability and altered LAMP-2A lysosomal dynamics - has been 
described upon sustained dietary lipid challenges (high fat or cholesterol diets), diabetes and in aging, all major risk factors for CVD $25,28-30$.

The protective role of CMA against mechanisms related to the etiology of CVD motivated us to investigate the possible contribution of CMA failure to the development of atherosclerosis ${ }^{31}$. Here, we show that blockage of CMA in mice increases their vulnerability to pro-atherosclerotic challenges, through both systemic and cell-autonomous changes in SMC and macrophages, the two main cell types involved in atherogenesis. Loss of CMA in SMC promotes their dedifferentiation and higher susceptibility to lipid challenges, while defective CMA in macrophages leads to a more pro-inflammatory phenotype. We propose that CMA is a defense mechanism activated in the vasculature in response to proatherosclerotic challenges and that reduced CMA activity leaves the vasculature vulnerable to these challenges. Using mice with genetically enhanced CMA, we demonstrate that, when exposed to pro-atherosclerotic challenges, they display reduced disease severity and slower progression. Our findings support that CMA could be a therapeutic target in atherosclerosis. 


\section{2 | CMA in atherosclerosis}

\section{Methods}

\section{Animal models and treatments}

KFERQ-PS-Dendra2 mice $^{32}$ were generated by donor egg injection in wild type FVB mice using the pRP.ExSi plasmid backbone with the insert coding for 11 amino acids including the KFERQ sequence of RNase $A$ in frame with the sequence of Dendra2 under the hybrid promoter CAGG and crossed back more than 9 generations to C57BL/6J. Male C57BL/6J LAMP-2A knock-out (L2AKO) were generated as described before ${ }^{21}$. C57BL/6J mice conditionally expressing hLAMP-2A ( $\mathrm{hL2AOE})^{33}$ were generated by inserting the hLAMP-2A CDNA sequence with a STOP cassette (a neo cassette flanked by two Loxp sites) into mouse ROSA26 locus in PTL1 (129B6 hybrid) ES cells that were used to generate heterozygous mice carrying the ROSA26-STOP-hLAMP-2A allele. Crossing these mice with ${ }^{\text {Tmx }}$ ER-Cre mice generated a mouse line in which expression of hLAMP-2A could be induced by injection of tamoxifen (TMX) (4 intraperitoneal (i.p.) injections of $20 \mathrm{mg} / \mathrm{kg}$ b.w. on alternate days) (Supplemental (S) Figure S1D) ${ }^{33}$. Male mice (KFERQ-PS-Dendra2, WT, L2AKO, CTRL and $\mathrm{hL2AOE}$ ) were intraorbitally injected at 12 weeks of age with a single dose of AAV8-PCSK9 $\left(1.0 \times 10^{11} \mathrm{VC}\right)$ to promote the degradation of low density lipoprotein receptor (LDLR) and increase circulating cholesterol levels ${ }^{34}$. Atherosclerosis was further induced by feeding the mice a Western-type diet (WD; D12108; ResearchDiets; saturated fats (35 kcal\%), cholesterol $(1.25 \% \mathrm{w} / \mathrm{w})$ and cholic acid $(0.22 \% \mathrm{w} / \mathrm{w}))$ for 12 weeks. KFERQ-PS-Dendra2, WT and L2AKO mice were fed a WD for 12 weeks and sacrificed for further analysis. After 6 weeks on diet, when we observed the drop in CMA activity, CTRL and hL2AOEwere injected with TMX to activate expression of hLAMP-2A and all mice were monitored for 10 additional weeks (Figure S7A). This longer protocol was required to compensate for the reduced intestinal absorption of cholesterol observed in the first 2 weeks upon TMX injection $^{35}$. For leupeptin treatment, mice were i.p. injected with leupeptin $(30 \mathrm{mg} / \mathrm{kg} \mathrm{b.w.;}$ Sigma, L5793) or PBS single injection $12 \mathrm{~h}$ and $2 \mathrm{~h}$ before euthanizing. Genotyping, breeding and treatments in this study were done accordingly to protocol and all animal studies were under an animal study protocol approved by the Institutional Animal Care and Use Committee of Albert Einstein College of Medicine.

\section{Antibodies}

Primary antibodies were from the following sources (dilutions, commercial source and catalog number indicated in brackets): rat anti-LAMP2 (1/500, Hybridoma Bank, GL2A7), rabbit anti-LAMP-2A (1/5000, Thermo Scientific, 512200), rabbit anti-LAMP-2B (in-house), rabbit anti-LDLR (1/1000, Abcam, ab52818), rabbit anti-human LAMP-2A (1/1000, Abcam, ab18528), rat anti-LAMP1 (1/500, Hybridoma Bank, 1D4B), mouse anti-human LAMP2 (1/500, Hybridoma Bank, H4B4), rabbit anti-LC3 (1/1000, Cell Signaling, 2775), rabbit antiP62 (Enzo Life Sciences BMLPW98600100), mouse anti- $\beta$-actin (1/10000 Sigma, A4700), 
anti-CD68 ( rat, 1/200 Bio-Rad, mca1957; rabbit, ab125212, Abcam), anti- $\alpha$-SMA (rabbit, 1/500 Abcam, ab202510; mouse, F3777, Sigma; mouse, M0851, Dako), rabbit anti-GBA (1/1000, Sigma, G4171), goat anti-Cathepsin D (1/500, Santa Cruz, sc-6486), mouse IgM anti-HSC70 (1/5000, Novus Biologicals, nb120-2788), mouse anti-GAPDH (1/3000, Abcam, ab8245), rabbit anti-iNOS (1/1000, Cell Signaling 2977), rabbit anti-COX-2 (1/1000, Cell Signaling, 12282), mouse anti-p53 (1/1000, Cell Signaling, 2524), rabbit anti-P27 (1/1000, Cell Signaling, 2552), rabbit, anti-P21 (1/1000, Abcam, ab109199) and anti-HMGB1 (1/1000, Abcam, ab18256). All the secondary antibodies were from Thermo Scientific. All antibodies used in this study were from commercial sources and were validated following the multiple dilution method and, where available, using cell lines or tissues from animals knock-out for the antigen. Sources of chemicals were as described before ${ }^{21,22}$.

\section{Cell culture and treatments}

SMC were isolated from 8 weeks old mice aortas (pool of 5 mice per genotype) by collagenase digestion and maintained in DMEM (Gibco) supplemented with $20 \%$ fetal bovine serum (FBS, Gibco), $2 \mathrm{mM}$ L-glutamine, $100 \mathrm{U} / \mathrm{ml}$ penicillin and $100 \mu \mathrm{g} / \mathrm{ml}$ streptomycin (Invitrogen). LPDS was prepared from FBS delipidated with $4 \%$ fumed silica ${ }^{36}$. SMC were incubated for 24h in DMEM media plus $5 \%$ LPDS with or without with or without LDL $\left(150 \mu \mathrm{g} / \mathrm{ml}\right.$ LDL-cholesterol) plus $0.1 \mathrm{U} / \mathrm{ml}$ of bovine lipoprotein lipase ${ }^{37}$. For Dil-LDL production, LDL was labeled with the fluorescent probe Dil (Invitrogen) as previously described ${ }^{38}$.

Bone marrow derived macrophages (BMDM) were isolated from 8 weeks old mice and differentiated in non-treated tissue culture plates by using Iscove's Modified Dulbecco's medium (IMDM) containing $20 \%$ of FBS and supplemented with $20 \%$ of L-929 cells conditioned media. After 5 days in culture, nonadherent cells were eliminated and adherent cells were trypsinized and seeded into the final plates for treatment ${ }^{36}$. Macrophage stimulation was attained by removing the culture medium and culturing cells for an additional $18 \mathrm{~h}$ in DMEM supplemented with $5 \%$ FBS (for CTRL) or $5 \% \mathrm{FBS}, 20 \mathrm{ng} / \mathrm{ml} \mathrm{IFN-}-$, and $100 \mathrm{ng} / \mathrm{ml}$ LPS (for IFN- $\mathrm{\gamma} /$ LPS) ${ }^{39}$.

\section{Tissue dissection and Histological Procedures}

All mice were euthanized with a pentobarbital overdose $(100 \mathrm{mg} / \mathrm{kg}$ i.p.) and blood was withdrawn via the right ventricle for flow cytometry and biochemical analysis. Mice were perfused via the left cardiac ventricle with PBS containing sodium nitroprusside $(0.1 \mathrm{mg} / \mathrm{ml}$; Millipore). Aortic arch and organs of interest were dissected and fixed in 1\% PFA overnight and paraffin-embedded. Aortic roots were serially sectioned and stained with hematoxylin and eosin (H\&E) for plaque area and necrotic core content. Five consecutive H\&E sections at $20 \mu \mathrm{m}$ intervals were analyzed blindly using computerized morphometry (Leica QWin V3) and averaged per mouse. A $100 \mu \mathrm{m}$ interval where a fully developed media within the aortic 


\section{2 | CMA in atherosclerosis}

valves was present was determined for each mouse. Sections within this $100 \mu \mathrm{m}$ interval were used for immunohistochemical staining. Two sections per mouse were stained with Sirius Red for collagen quantification and averaged per mouse. Immunostaining for LAMP$2 \mathrm{~A}$ in mouse aorta was performed using a rabbit anti-LAMP-2A (Thermo scientific; 512200) following standard procedures. Macrophages and SMC were immunostained using rabbit anti-CD68 (ab125212, Abcam) antibody followed by secondary anti-rabbit HRP-labeled antibody (DPVR-55-HRP, Immunologic) and anti-aSMA (F3777, Sigma) followed by secondary-HRP-labelled antibody (11.426.346.910, Roche), respectively. Epitope-antibody binding was visualized as a brown precipitate using diaminobenzidine. Alizarin red (A553325G, Sigma) was used to detect calcification. For colocalization staining, primary antibodies, followed by secondary anti-rabbit AP-labelled antibody (DPVR-55-AP, Immunologic) were used in CD68 and LAMP-2A staining, and for SMC staining mouse anti- $\alpha$ SMA (M0851, Dako) primary antibody, followed by secondary anti-mouse Biotin-labelled antibody (RPN1001v1, Amersham) and ABC-AP amplification (AK-5000, Vector) were used. Epitope-antibody binding was visualized as either blue or red precipitate using Vector substrate kits (SK-5300 or SK-51000, respectively).

\section{Lipid analysis}

Plasma was obtained by centrifugation at $6,000 \mathrm{~g}$ for $10 \mathrm{~min}$ at $4^{\circ} \mathrm{C}$, snap-frozen and stored at $-80^{\circ} \mathrm{C}$ until further use. Plasma total cholesterol (TC) (Cholesterol E, 999-02601, Wako) and triglycerides (TG) (L-Type Triglyceride M, 992-02892 and 998-02992, Wako) were assessed using standard enzymatic assays automated on the Infinite ${ }^{\circledR} 200$-Pro (Tecan).

Pooled plasma samples from mice according to genotype and treatment were used for lipoprotein fractioning on a Superose 6 PC 3.2/30 column (ÄAkta System, Amersham Pharmacia Biotech). The samples were eluted at a constant flow rate of $50 \mu \mathrm{l} / \mathrm{min}$ in PBS ( $\mathrm{pH}$ 7.4). Fractions of $50 \mu$ l were collected and assayed for TC and TG using the kits described above. Plasma levels of insulin and PAI-1 were measured using ELISA (90080, Crystal Chemicals and ab197752, Abcam). Plasma levels of CCL3, CCL4 and G-CSF were measured using MILLIPLEX MAP Mouse Cytokine/Chemokine kit (Millipore) in a Luminex Magpix (Luminex).

\section{Metabolic analysis}

Body weight was measured weekly during the study period. Body composition was determined on 21-week-old mice by magnetic resonance spectroscopy (MRS) using an echo MRS instrument (Echo Medical System) and metabolic measurements (oxygen consumption, carbon dioxide production, food intake, and locomotor activity) were obtained continuously every 8 minutes using a CLAMS (Columbus Instruments) open-circuit indirect calorimetry system for 8 days. Insulin resistance was measured with an intraperitoneal insulin tolerance test (ITT) performed on 21-week-old mice after $4 \mathrm{~h}$ fasting. 
Blood glucose was measured before i.p. of insulin (1.5 U/kg body weight) and then 15,30 , 45, 60, 90, 120 and 150 minutes after injection.

\section{Flow cytometry and serum parameters}

Whole blood cell composition was analyzed using flow cytometry after erythrocyte lysis and incubation with the following specific antibodies to detect leucocyte subsets: leucocytes (CD45+; 103129, Biolegend), T cells (CD3ع+, NK1-1-; 48-0032-80, ThermoFisher), T helper cells (CD4+; 15-0041-81, ThermoFisher), cytotoxic $T$ cells (CD8a+; 11-0081-82, ThermoFisher), B cells (CD45R/B220+; 561227, BD), NK cells (NK1-1+; 561046, BD), granulocytes (CD11bhigh Ly6Ghigh; 11-0112-41 ThermoFisher, 560600, BD, respectively) and monocytes (CD11bhigh Ly6Glow Ly6Chigh/intermediate/low) using the Absolute Counting Tubes (340334, BD Trucount ${ }^{\mathrm{TM}}$ ). Data were acquired using a Becton Dickinson LSRII-U and analyzed with FACSdiva software (BD) and gating strategy is depicted in Supplemental Figure S1.

\section{Human carotid plaque analysis}

Human carotid autopsy samples ( $n=38$, mean age 72 years, $64 \%$ men) representing the following stages of atherosclerosis: intimal thickening, pathological intimal thickening, thick fibrous cap (stable) atheroma, and plaque with intraplaque hemorrhage, were obtained and processed for immunohistochemistry as follows. After antigen retrieval (target retrieval DAKO), slides were incubated overnight with primary antibody (Human LAMP-2A (ab18528, Abcam), CD68 (macrophage marker, ab125212, Abcam) or aSMA (SMC marker, F3777, Sigma)), followed by secondary-biotin-labelled antibodies, and ABC-HP amplification (Vector). Epitope-antibody binding was visualized as a brown precipitate using diaminobenzidine. Colocalization of LAMP-2A and CD68 and $\alpha$ SMA was done on directly adjacent sections using rabbit anti-LAMP-2A (18528, Abcam), mouse anti-CD68 (M0814, Dako) and mouse anti- $\alpha$ SMA (M0851, Dako) primary antibodies respectively, followed by either anti-mouse or anti-rabbit AP-labelled secondary antibody (DPVM-55-AP or DPVR-55$A P$, Immunologic). Epitope-antibody binding was visualized as either a blue (LAMP-2A) or red (CD68/aSMA) precipitation using Vector substrate kits (SK-5300 or SK-51000 respectively).

LAMP-2A mRNA expression in thick fibrous cap atheromas and intraplaque hemorrhagerich plaques was analyzed by microarray derived from paired segments of the same patient undergoing carotid endarterectomy ${ }^{40}$. In short, flanking sites of tissue used for transcriptomics analysis were formalin-fixed for subsequent plaque stage analysis. LAMP2A mRNA expression intensities from microarrays were correlated with morphometrically analyzed histological plaque characteristics: plaque size, necrotic core (\% of plaque) and macrophages (\% CD68 of plaque). Classification of all used plaques was performed on H\&E stained slides according to Virmani et $a l^{12}$, by experienced cardiovascular pathologists (JCS, 


\section{2 | CMA in atherosclerosis}

$M G)$. All experiments were conducted in agreement with the code for proper secondary use of human tissue in the Netherlands (http://www.fmwv.nl). This study complies with the Declaration of Helsinki, and the local Medical Ethical Committee in accordance with national regulations approved use of this tissue (protocol number 16-4-181 and 03-1140).

LAMP-2 protein expression in human atherosclerotic plaque was analyzed using the AtheroExpress Study biobank, in which plaques from patients undergoing carotid endarterectomy were obtained. Patients were included if they suffered a symptomatic ischemic event within 6 months prior to the surgery. After surgery, patients were followed up for a minimum of three years to assess if they underwent a secondary major adverse cardiovascular event or not. Lysates of these plaques were used for western blot analysis.

\section{CMA activity}

CMA activity in vivo was determined in aorta arches from KFERQ-Dendra2 mice using CT embedding method as following: Aortas were fixed for $12 \mathrm{~h}$ at $4^{\circ} \mathrm{C}$ in fixation buffer $(2 \%$ formaldehyde, $0.2 \%$ picric acid in PBS, pH7.0) and then washed with $70 \%$ ethanol, followed by two washes in PBS. Tissues were immersed in 30\% sucrose and then embedded in OCT for sectioning in a cryostat (Leica CM3050 S). After air-drying for $30 \mathrm{~min}$, sections were stored at $-20^{\circ} \mathrm{C}$ until use. Colocalization of LAMP1, CD68 and $\alpha$ SMA was done on sequential serial sections using rat anti-LAMP-1 (Hybridoma Bank, 1D4B), rat anti-CD68 (Bio-Rad, mca1957) and rabbit anti- $\alpha$ SMA (Abcam, ab202510, alexa 594-labelled). Slices were mounted in DAPI-Fluoromount-G to highlight the cell nucleus. Direct fluorescence images were obtained with a confocal microscope (TCS SP5; Leica) using an HCX Plan Apo CS 63.0x 1.40 NA oil objective in the Leica Application Suite X (LAS X) or an Olympus FV1000 multiphoton microscope with a $25 \times 1.05 \mathrm{NA}$ water immersion objective as previously described ${ }^{41}$. Collagen was visualized by second harmonic generation.

CMA activity in cultured SMC was measured using lentivirus-mediated expression of fluorescent photoswitchable KFERQ-PSDendra2 reporter $^{42}$. Cells were photoswitched with a 405nm light emitting diode (LED: Norlux) for $3 \mathrm{~min}$ with $3.5 \mathrm{~mA}$ (constant current) and 16 $\mathrm{h}$ later fixed with $1 \%$ paraformaldehyde. Images were acquired with an Axiovert 200 fluorescence microscope (Carl Zeiss), with 1.4 numerical aperture. The average number of fluorescent puncta per cell was quantified using Image $\mathrm{J}(\mathrm{NIH})$ in individual single planar images after thresholding. Values are presented as number of puncta per cell section that in our acquisition conditions represents $10-20 \%$ of the total puncta per cell ${ }^{43}$.

\section{Lysosomal isolation}

Lysosomes were isolated from BMDM after disruption of the plasma membrane by nitrogen cavitation and sequential centrifugation in Percoll/metrizamide discontinuous density gradients $^{44}$. Preparations with more than $10 \%$ broken lysosomes, measured by $\beta$ - 
hexosaminidase latency, were discarded ${ }^{44}$.

\section{Microarray}

Total RNA from a pool of 3 independent experiments with SMC in culture was extracted using TRIzol ${ }^{\circledR}$ (Invitrogen) and purified with RNeasy chromatography (Qiagen) in LPDS media and LPDS plus LDL $(150 \mu \mathrm{g} / \mathrm{ml})$. Cy3-labeled RNA $(0.6 \mu \mathrm{g})$ from each condition were hybridized to Agilent Mouse $8 \times 60 \mathrm{~K}$. Data were processed using the oligo package and normalized using Robust Multiarray Average (RMA) method. Gene set was filtered to remove genes without Entrez or GO annotation (21912 genes out of 55682) and genes with an IQR $>0.5$. The full microarray raw data has been deposited in GEO accession number GSE49553. Pathway analysis was performed using the IPA software (Ingenuity Systems) and STRING database (https://string-db.org/). Validation of the microarray was performed in a subset of genes (Figure S4C).

\section{Quantitative Proteomics and Protein Pathway Analysis}

BMDM lysosomes active for CMA were isolated from WT and L2AKO macrophages treated in CTRL or stimulated with IFN $/$ /LPS and treated or not (untreated) with $2 \mathrm{mM} \mathrm{NH}_{4} \mathrm{Cl}$ and $100 \mu \mathrm{M}$ leupeptin 12 hours before isolation. Lysosomes from three different sets were pooled and analyzed for purity, integrity, ponceau red electrophoretic patterning and enrichment in markers of CMA lysosomes by immunoblot (Figure S5C). Quantitative proteomics analysis was performed in the two different genotypes under the four different conditions using isobaric tags for relative and absolute quantitation (iTRAQ) by Applied Biomics, Inc.. For each sample (10 $\mathrm{g}$ g of protein), the buffer was replaced with $0.5 \mathrm{M}$ triethylammonium bicarbonate, $\mathrm{pH} 8.5$, followed by reduction, alkylation, trypsin digestion, iTRAQ labeling, and sample clean-up according to the manufacturer's instructions ( $A B$ SCIEX). NanoLC was carried out using a Dionex Ultimate 3000 (Milford, MA). Tryptic peptides were loaded into a $\mu$-Precolumn Cartridge and separated on an acetonitrile gradient (ranging from $5 \%$ to $60 \%$ ) on a C18 Nano LC column. Fractions were collected at 20-second intervals followed by Mass Spectrometry analysis on AB SCIEX TOF/TOF ${ }^{\mathrm{TM}} 5800$ System (AB SCIEX). Mass spectra were acquired in reflectron positive ion mode. TOF/TOF tandem MS fragmentation spectra were acquired for each ion, averaging 4,000 laser shots per fragmentation spectrum on (excluding trypsin autolytic peptides and other known background ions). The resulting fragmentation spectra were submitted to MASCOT search engine (version 2.3, Matrix Science) to search the database of National Center for Biotechnology Information non-redundant (NCBInr). Searches were performed without constraining protein molecular weight or isoelectric point, with variable methyl-thiolation of cysteine and oxidation of methionine residues, fixed $\mathrm{N}$-terminal- and lysine-modifications with ITRAQ labels, and one missed cleavage. Quantitation was performed on peptides displaying an ion score confidence interval of 95 percent or higher. 


\section{2 | CMA in atherosclerosis}

For each protein hit the average ratio(s) for the protein, the number of peptide ratios that contributed, and the geometric standard deviation were determined. Values in the L2AKO untreated experimental groups were compared to their respective $\mathrm{NH}_{4} \mathrm{Cl} /$ leupeptin treated samples and then with their respective WT groups and are represented as the average of folds (lysosomes isolated from untreated WT BMDM are given a value of 1). CMA substrate proteins were defined as those for which leupeptin treatment resulted in increase in lysosomal levels $>10 \%$ and with a reduction in $\mathrm{NH}_{4} \mathrm{Cl}$ /leupeptin response of $>10 \%$ in the L2AKO. Validation of the subset of proteins of interest was performed by immunoblotting in lysosomal fractions isolated from independent BMDM (not shown). The protein sets catalogued as CMA substrates from the ITRAQ experiments were analyzed using the IPA software (Ingenuity Systems) and STRING database (https://string-db.org/).

\section{Real-Time Quantitative-Polymerase Chain Reaction}

Total RNA was isolated from cells using TRIzol ${ }^{\circledR}$ Reagent (Invitrogen). RNA (1 $\mu \mathrm{g}$ ) was used to perform the reverse transcription with High Capacity cDNA Archive Kit (Applied Biosystems). Real-time PCR reactions were performed on an ABI Prism 7500 sequence detection PCR system (Applied Biosystems) according to manufacturer's protocol, using the $\Delta \Delta C$ t method as described ${ }^{45}$. Quantification of mRNA levels was done by amplification of cDNA using Power SYBR Green PCR Master Mix (4368702, ThermoFisher). The primer sequences are listed in Supplemental Table S1. Expression levels are given as ratio to housekeeping gene HPRT1 and data is expressed as fold vs basal values.

\section{Other methods}

Rates of cellular proliferation were determined as incorporation of BrdU 24h hours after plating (11444611001, Sigma) and cytotoxicity was determined by ApoTox-Glo Triplex Assay (G6320, Promega). Electrophoresis and immunoblot were performed using nitrocellulose membranes after cell lysis in $0.25 \mathrm{M}$ sucrose buffer $(\mathrm{pH} 7.2)$ containing protease and phosphatase inhibitors. Cell lysates were centrifuged at increasing speeds to discard intact cells $(300 \mathrm{~g}, 10 \mathrm{~min})$ and dead cells $(2,000 \mathrm{~g}, 10 \mathrm{~min})$. After electrophoresis and transfer to nitrocellulose membrane, the proteins of interest were visualized by incubation of the membranes with the primary and corresponding secondary antibody by chemiluminescence using peroxidase-conjugated secondary antibodies in G-BOX Chemi XX6 (Imgen). $\beta$-actin and red ponceau were used as loading control. Macroautophagy was measured upon transduction of cells with a lentiviral vector expressing mCherry-GPF-LC3 ${ }^{46}$, as the conversion of dual fluorescence puncta (autophagosomes) into only red fluorescent puncta (autolysosomes). Immunoblot for LC3 and P62/SQSTM1 in cells incubated for $12 \mathrm{~h}$ with $\mathrm{NH}_{4} \mathrm{Cl} /$ leupeptin was used to analyze macroautophagy flux. 


\section{Statistics}

All data are presented as individual values (symbols) and/or mean+SEM, with *p $<0.05$, $* * p<0.01, * * * p<0.001$ and $* * * * p<0.0001$. For in vitro assays we determined the number of experimental repetitions to account for technical variability and changes in culture conditions. In all instances, " $n$ " refers to individual experiments or animals. Data comes from a minimum of three independent experiments and instances with higher number of repetitions are indicated in the figure legends. The number of animals used per experiment was calculated through power analysis based in previous results. Animals were randomly attributed to control or treatment groups. No mouse was excluded from the analysis unless there were technical reasons or the mouse was determined to be in very poor health by the veterinarian. Outliers were determined by the ROUT method $(Q=1 \%)$. Investigators were blinded to the treatment during data collection and analysis and unblinding was done when the analysis was completed for plotting. If not indicated otherwise, all parameters were analyzed using independent sample tests and were tested for normal distribution using Shapiro-Wilk normality test. Parameters with two groups were compared with student's ttest or Mann-Whitney rank-sum test, depending on outcome of the D'Agostino \& Pearson normality test. In case of more than two groups, parameters were analyzed using two-way ANOVA followed by Bonferroni's Multiple Comparison Test or Kruskal-Wallis rank-sum test, followed by Dunn's post-hoc testing, in the absence of a normal distribution. CMA activity measured by KFERQ-Dendra2 puncta in SMC was subjected to Analysis of Variance (Oneway ANOVA) followed by Tukey's post hoc tests. Time course experiments were analyzed using repeated measured (mixed model) ANOVA, followed by Bonferroni post-tests upon confirmation of homogeneity of variances using Levene's (GraphPad 7.0). Correlation analysis was performed using Spearman bivariate correlation analysis (IBM SPSS statistics 22). 


\section{2 | CMA in atherosclerosis}

\section{Results}

\section{CMA blockage exacerbates atherosclerosis in a murine experimental model}

Since no information is available on the status of CMA in the vasculature and its possible changes during atherosclerotic plaque development, we used the recently developed transgenic mice expressing a fluorescence reporter for CMA (KFERQ-PS-Dendra2 mice) that allows measuring CMA activity in vivo ${ }^{32}$. When this artificial CMA substrate is delivered to lysosomes, CMA activity is detected as fluorescent puncta against the diffuse fluorescent cytosolic pattern ${ }^{32}$. Using aortas from these mice, we found that a fraction of lysosomes (highlighted with the endolysosomal marker LAMP1) were positive for the reporter both in cells in the media (SMC) and, to a less extent, in the intima (endothelial cells), in support of active CMA in the vasculature under basal conditions (Figure 1A and Supplemental (S) Figure S1A). When we promoted atherosclerosis development in KFERQ-PS-Dendra2 mice through hypercholesterolemia (using injection of adeno-associated virus 8-mediated overexpression of proprotein convertase subtilisin/kexin type 9 (AAV8-PCSK9) and a high cholesterol-containing diet (Western type diet, WD) for 12 weeks), aortas from these mice revealed a marked reduction in the number of fluorescent puncta that was almost absent in the plaque (Figure 1A and Figure S1A). Co-staining of these atherosclerotic aortas with the SMC marker $\alpha$-smooth muscle actin ( $\alpha$-sma) and the macrophage marker cluster of differentiation 68 (CD68), revealed almost no CMA activity in either cell type (Figure 1B and S1B). Immunohistochemistry of the plaque demonstrated that levels of LAMP-2A, limiting component for CMA, can be detected both in SMC and macrophages by 6 weeks of plaque formation, but LAMP-2A levels significantly decreased in more advanced stages (12 weeks) of murine atherosclerotic disease, in agreement with the observed reduction in CMA activity (Figure $\mathbf{1 C}$ and S1C).

To determine if the initial upregulation of LAMP-2A in response to the dietary challenge was protective and whether reduced CMA contributes to disease progression, we used a mouse model with systemic blockage of CMA (constitutive knock-out for LAMP-2A, L2AKO $^{47}$ ) (Figure S1D). At 3 months of age, L2AKO mice on chow diet display slightly lower body weight and higher circulating total cholesterol levels than wild-type (WT) littermates (Figure S1E-F). When L2AKO mice were fed WD for 12 weeks, we observed a marked increase in total circulating cholesterol and triglyceride (TG) levels (Figure 1DE), mainly in the very-low-density lipoprotein (VLDL) and LDL fractions (Figure 1F-G). Atherosclerotic plaques in the aortic root of L2AKO mice were larger than in WT mice (approx. 39\%) (Figure $\mathbf{1 H}-\mathbf{I}$ ), with a noticeable trend toward bigger necrotic cores, and significantly more advanced plaques (Figure $\mathbf{1 J - K}$ ). Plaques in the CMA-incompetent mice had more collagen content, thicker fibrous cap and higher abundance of calcifications (Figure $\mathbf{1 L - O}$ and Figure $\mathbf{S 1 G}$ ). In addition, the relative content of both 
ACTA2 for contractile SMC and CD68+ for macrophages at the plaque was significantly lower in L2AKO mice (Figure 1P-Q).

Overall, reduced CMA activity associates with more severe atherosclerotic pathology supporting an anti-atherosclerotic protective function for CMA. 

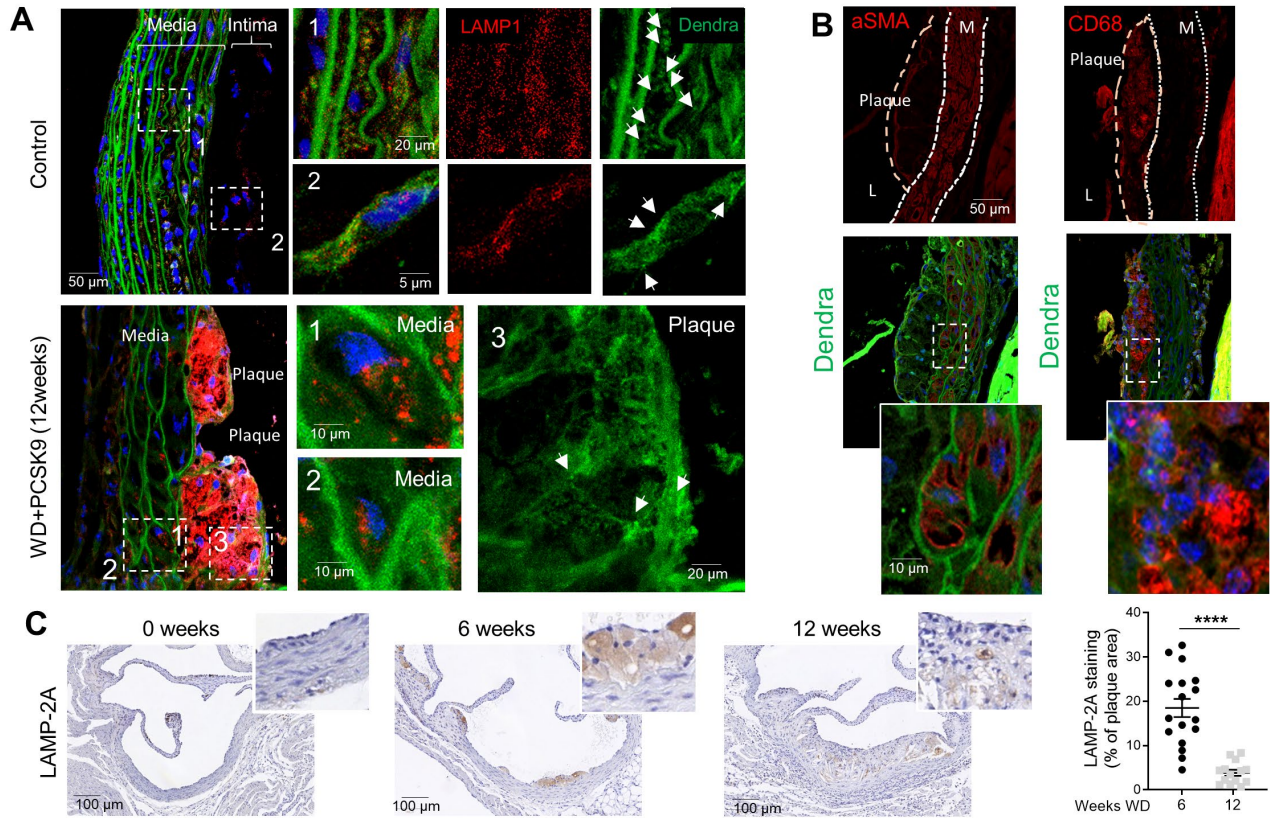
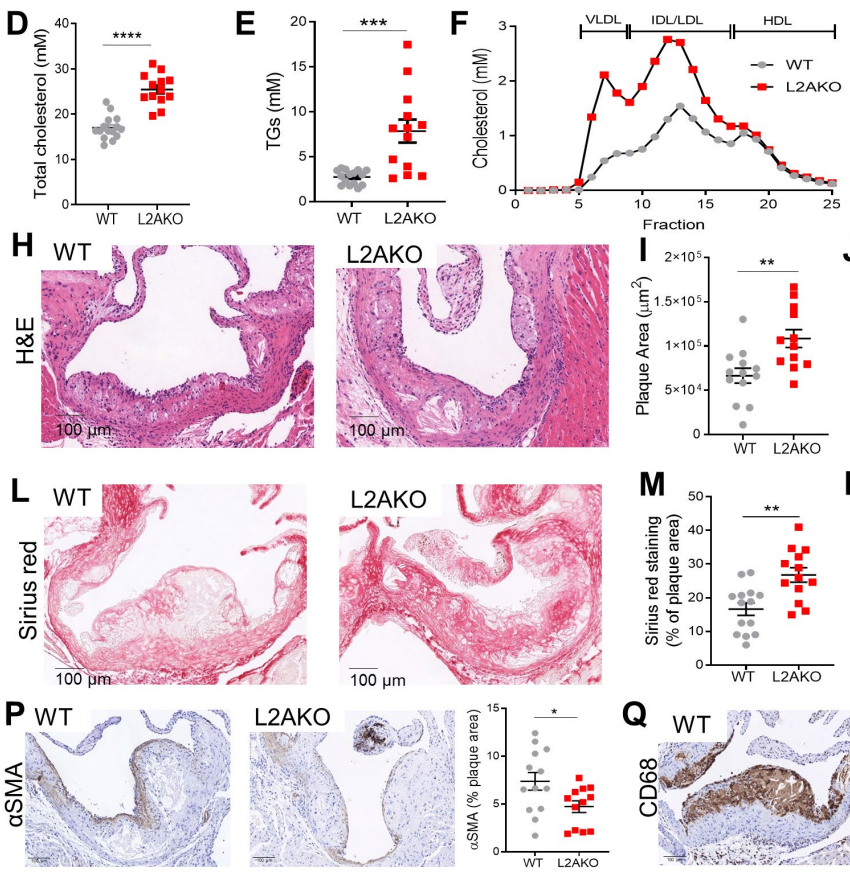
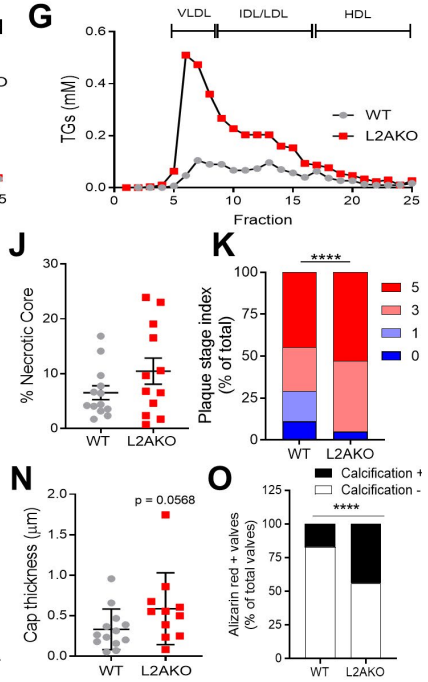

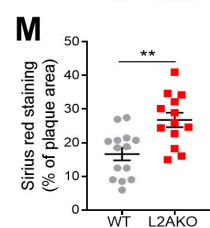

Q WT

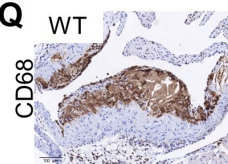

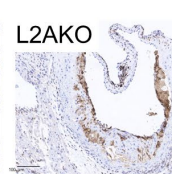

Figure 1. CMA deficiency aggravates atherosclerosis in a murine experimental model. A. CMA activity in aorta from KFERQ-Dendra2 mice untreated (Control) or subjected to a pro-atherosclerotic treatment (injected with AAV8 PCSK9 and maintained for 12 weeks on Western-type diet, WD). Representative images of aorta sections co-stained with LAMP1 to highlight endolysosomal compartments. Insets: boxed areas at higher magnification. Arrows: fluorescent puncta. B. Co-staining 
of aortas from the same treated mice with $\alpha$-SMA or CD68. Individual and merge channels are shown. C. Levels of LAMP-2A at the indicated times of the pro-atherosclerotic intervention. Representative images of aorta sections (left) and quantification in the neointima (right). $n=18$. D-G. Circulating lipids in wild type (WT) and LAMP-2A null mice (L2AKO) subjected to the pro-atherosclerotic challenge for 12 weeks. Circulating total cholesterol (D), triglycerides (TG) (E), cholesterol profile (F) and TG profile (G). Individual values (D, E) and average curves $(\mathbf{F}, \mathbf{G})$ shown. $\mathrm{n}=13$. $\mathbf{H}-\mathbf{O}$. Plaque properties in the same mouse groups. Representative images of aortas stained for H\&E $(\mathbf{H})$ or sirius red $(\mathbf{L})$ and quantification of plaque area (I), size of the necrotic core $(\mathbf{J})$, plaque stage index $(\mathbf{K})$, sirius red positive area $(\mathbf{M})$ and cap thickness $(\mathbf{N})$. Calcification analysis in aortas stained for alizarin red $(\mathbf{O})$ as shown in Supplemental Figure 1g. $n=16$. $P, Q$. Representative images of aortas immunostained for $\alpha \mathrm{SMA}+(\mathrm{SMC})(\mathbf{P})$ and CD68 macrophages $(\mathbf{Q})$ and quantification of stained area (right) $n=16$. Individual values (symbols) and mean \pm SEM are shown. Experiments in panels $\mathbf{A}$ and $\mathbf{B}$, were repeated 3 times with similar results. All data were tested for normal distribution using D'Agostino and Pearson normality test. Variables that did not pass normality test were subsequently analyzed using Mann-Whitney rank-sum test. All other variables were tested with the Student's t-test. ${ }^{*} p<0.05,{ }^{* *} p<0.01,{ }^{* * *} p<0.005$ and ${ }^{* * * *} p<0.0001$.

\section{Metabolic dysfunction in CMA-deficient mice}

To determine the basis for the protective effect of CMA against atherosclerosis, and because of the previously described regulation of hepatic glucose and lipid metabolism by $\mathrm{CMA}^{21}$, we evaluated metabolic parameters shown to be major risks factors in CVD. We found that L2AKO mice gained 50\% more body weight than the WT group during the 12 weeks of WD (Figure 2A), mostly due to a higher fat mass content (Figure 2B and Figure S2A). Indirect calorimetry revealed that the increased adiposity of L2AKO mice did not originate from higher food consumption (Figure 2C and Figure S2B-C), but it could be explained by reduced energy expenditure (Figure 2D and Figure S2D-E) and less physical activity (Figure $\mathbf{2 E}$ ). The decrease in respiratory exchange ratio (RER) - indicative of lipid use as energy - observed in WT mice on WD was significantly more pronounced in L2AKO mice (Figure S2F), suggesting impaired carbohydrate utilization in these mice. Indeed, L2AKO mice showed marked hyperinsulinemia (Figure 2F) and increased insulin resistance (Figure 2G-H), typical characteristics in $\mathrm{CVD}^{48}$. Circulating levels of the prothrombotic and pro-fibrotic cytokine plasminogen activator inhibitor type 1 (PAI-1) were also significantly higher in L2AKO mice (Figure 21). These findings support that loss of CMA accentuates the systemic derangements in metabolism and coagulation imposed by the WD, thus rendering organisms more prone to atherosclerosis. 
A

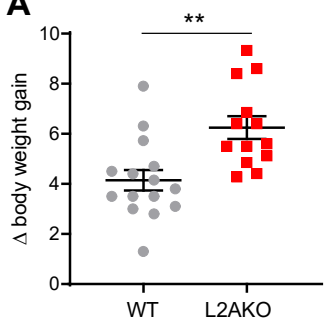

D
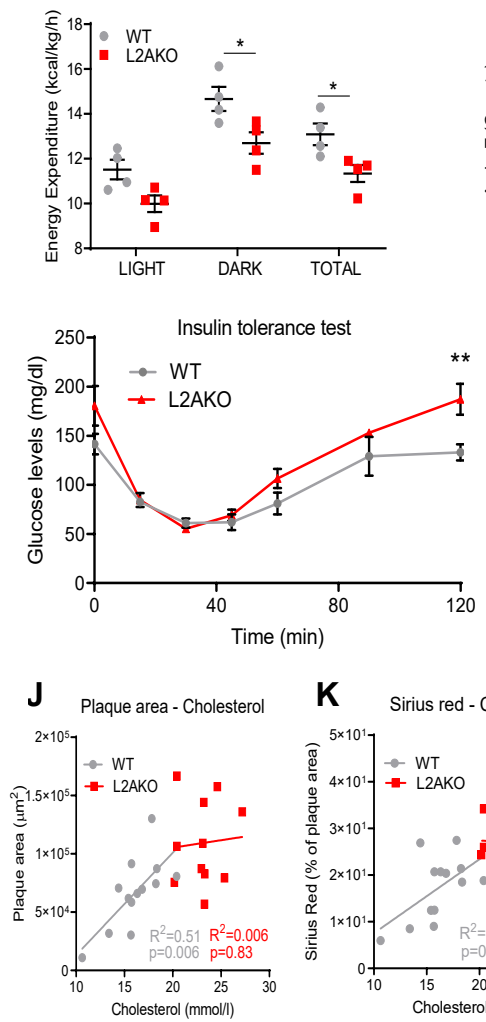

B

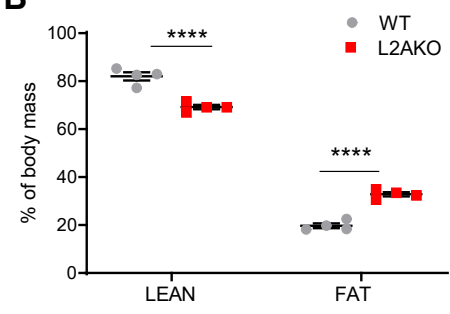

$E$

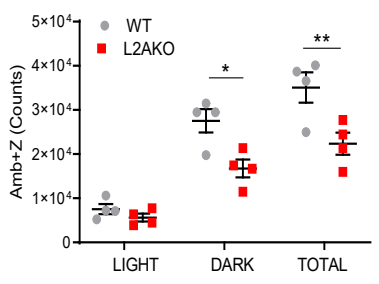

H

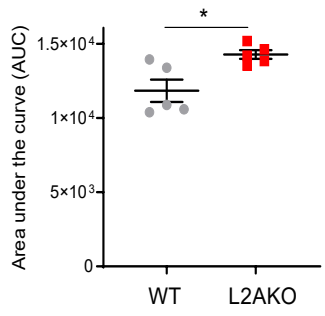

L CD68-Cholesterol

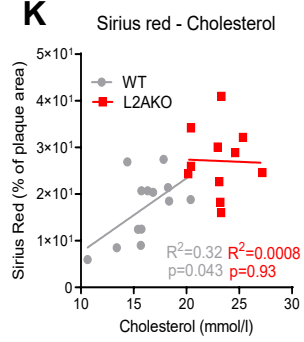

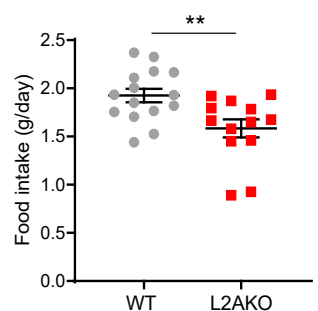

$\mathbf{F}$

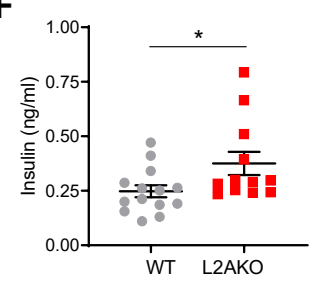

I

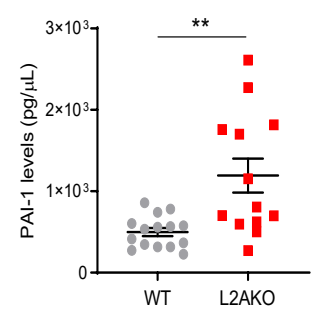

Figure 2. A pro-atherogenic challenge elicits metabolic dysfunction in CMA deficient mice. A-E. Wild type (WT) and LAMP-2A null mice (L2AKO) subjected to a pro-atherosclerotic treatment (injected with AAV8 PCSK9 and maintained for 12 weeks on the Western-type diet, WD) were compared for body weight gain ( $n=14$ WT, 16 L2AKO) (A), body composition ( $n=4)$ (Body composition: two-way ANOVA, $F=120.1 ; P<0.0001$ for interaction, $F=1736 ; P<0.0001$ for LEAN/FAT, $F=0.01544 ; P=0.9032$ for genotype, $n=4)(B)$, food intake $(n=14 W T, 16$ L2AKO) $(C)$, energy expenditure (two-way ANOVA, $F=0.1185 ; P=0.8889$ for interaction, $F=21.05 ; P<0.0001$ for light $/$ dark/total, $F=22.41 ; P=0.0002$ for genotype, $n=4)(D)$ and ambulatory parameters $(x+z$ axis) (Ambulatory parameters: two-way ANOVA, $F=3.194 ; P=0.0650$ for interaction, $F=49.75 ; P<0.0001$ for light $/$ dark $/$ total, $F=20.75 ; P=$ 0.0002 for genotype, $n=4)(E)$. F-I. Circulating levels in the same mouse groups of insulin ( $n=14$ WT, 16 L2AKO) (F), glucose during an insulin tolerance test (repeated measures two-way ANOVA after 
Bonferroni's post hoc test, $F=1.851 ; P=0.1090$ for interaction, $F=31.96 ; P<0.0001$ for time, $F=15.99 ; P=0.0040$ for genotype, $n=4)(G)$, area under the curve from the insulin tolerance test $(H)$ and circulating PAI-1 levels ( $n=14$ WT, 16 L2AKO) (I). J-M. Correlation between plasma cholesterol and different plaque parameters: plaque area (J), collagen (K), macrophages $(\mathbf{L})$ and SMC \% of plaque area (M) in the same mouse groups ( $n=14$ WT, 16 L2AKO). All data, when applicable, were tested for normal distribution using D'Agostino and Pearson normality test. Variables that did not pass normality test were subsequently analyzed using Mann-Whitney rank-sum test. All other variables were tested with the Student's t-test. Individual values (symbols) and mean \pm SEM are shown. ${ }^{*} p<0.05,{ }^{* *} p<0.01$ and $* * * \mathrm{p}<0.001$.

\section{CMA blockage promotes SMC dedifferentiation}

Whereas circulating cholesterol levels in WT mice show the previously described correlation with different plaque properties, such correlations are lost in L2AKO mice (Figure 2J-M). This suggests that factors other than systemic metabolic changes also contribute to the higher vulnerability of L2AKO mice to atherosclerosis. This motivated us to investigate whether local changes of CMA in the vasculature could contribute to disease progression.

We first examined CMA in primary cultured SMC exposed to a physiological lipid challenge (LDL loading) and found a dose-dependent upregulation of CMA followed by a gradual decrease, once toxic concentrations of LDL are reached (Figure $3 \mathbf{A}$ and Figure S3A). Exposure of L2AKO SMC to fluorescent LDL (diLDL) resulted in higher intracellular lipid accumulation (Figure 3 B) and reduced cellular viability as LDL concentrations increased (Figure $\mathbf{3 C}$ ). This higher susceptibility to lipotoxicity can be primarily attributed to the loss of CMA, since other types of autophagy (i.e. macroautophagy shown in S3B,C) were fully functional in these cells.

Comparative analysis of the transcriptional profile of WT and L2AKO SMC revealed marked differences under basal conditions and an inadequate transcriptional response after exposure to LDL in L2AKO cells (Figure 3D and Figure S4A-C). Under basal conditions L2AKO SMC exhibited loss of ACTA2, an activated-macrophage like gene profile and acquisition of recently identified markers of modified, dedifferentiated $\mathrm{SMC}^{9}$ (Figure 3D and S4D). These findings are in line with loss of ACTA2+ contractile SMCs in the plaques in vivo (Figure 1P). Gene set enrichment analysis (with STRING database) further showed upregulation of nodes related to cell migration, proliferation, differentiation and response to lipids (Figure S4E).

Loading with LDL, induced changes in genes related to lipid metabolism in both genotypes (Figure S4F), but we identified quantitative differences in this response. Thus, using Ingenuity Pathway Analysis (IPA), we found that L2AKO cells have a defective response to the lipid challenge with reduced upregulation of genes involved in the cholesterol pathway and display cholesterol as one of the top molecules upregulated in these cells (Figure S4G). The immune component of the response of SMC to lipids is also different in L2AKO cells. While WT cells orchestrate the well-characterized inflammatory response, the immune 


\section{2 | CMA in atherosclerosis}

response of L2AKO cells is mainly composed of genes related to leucocyte activation and cell migration (Figure S4F). Differential gene expression analysis and gene set enrichment upon lipid loading also identified gene nodes unique for L2AKO cells related with cell death and cellular response to stress, including the response to DNA damage (Figure 3E), which we experimentally confirmed to be significantly increased in these cells (Figure 3F-G). These findings support that failure to activate CMA in SMC makes them unable to adapt to the environmental lipid challenge, as previously described also in CMA-deficient hepatocytes ${ }^{21}$.

Analysis of upstream regulators of the group of genes differentially expressed in L2AKO SMC revealed as the top change a significant $\left(p<3.13 \times 10^{-46}\right)$ downregulation of the tumor protein 53 (p53) signaling pathway (Figure 3E). Immunoblot against different components of the p53 signaling pathways confirmed markedly reduced levels of p53 protein and of the cyclindependent kinase inhibitor $1 \mathrm{~A}$ (p21) in L2AKO SMC, whereas cyclin-dependent kinase inhibitor 1B (p27) content was higher in these cells compared with WT (Figure 3H). In light of the well-characterized role of p53 as anti-apoptotic molecule in response to lipid challenges, the identified defect in p53 signaling in L2AKO SMC provides an explanation for their higher death count (Figure 3C), increased DNA damage (Figure 3F-G) and higher proliferation rates (Figure 3I) upon LDL loading. Furthermore, relevant to this study is the fact that $\mathrm{p} 53$ has previously been shown to protect against SMC dedifferentiation ${ }^{49}$, already noticeable in L2AKO SMC under basal conditions (Figure 3D).

We also detected that L2AKO SMC show constitutively higher intracellular content of the pro-inflammatory and damage-danger-associated molecule pattern (DAMP) chaperone high mobility group box protein-1 (HMGB1) (Figure S4H), known to complex with p53 ${ }^{50,51}$ and to stimulate PAI-152. Even more striking was the augmented release of HMGB1 into the extracellular media in the form of large molecular weight complexes (Figure 3J). This continuous release of HMGB1 from L2AKO SMC in the arterial wall may be one of the major drivers of the local inflammation and calcium deposition observed in the aortas of L2AKO mice (Figure $\mathbf{1 0}$ and Figure S1G) and may also contribute to perpetuate dedifferentiation of CMA-defective SMC. Also, as part of the possible impact of L2AKO SMC in arterial wall, and in agreement with our in vivo observations (Figure 1K), we found a marked increase in most of collagen genes previously associated with plaque fibrosis (Figure 3K), which further supports SMC transition into a synthetic phenotype.

Our findings in L2AKO SMC confirm that fully functional CMA is required in their defense against lipotoxicity and to maintain the identity of SMC by preventing their dedifferentiation into secretory-migratory cells. 
A

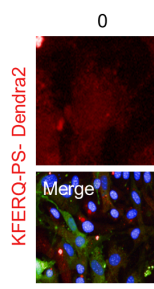

C

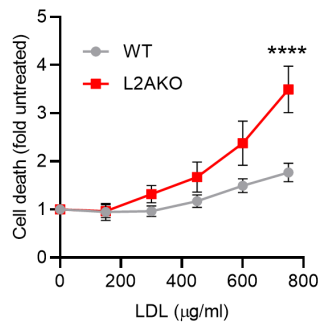

E

Different in L2AKO in response to LDL

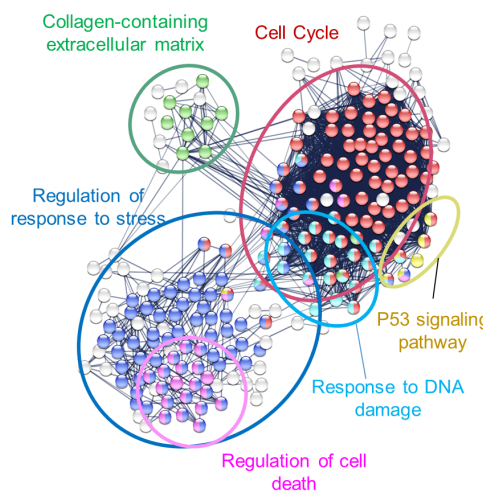

death
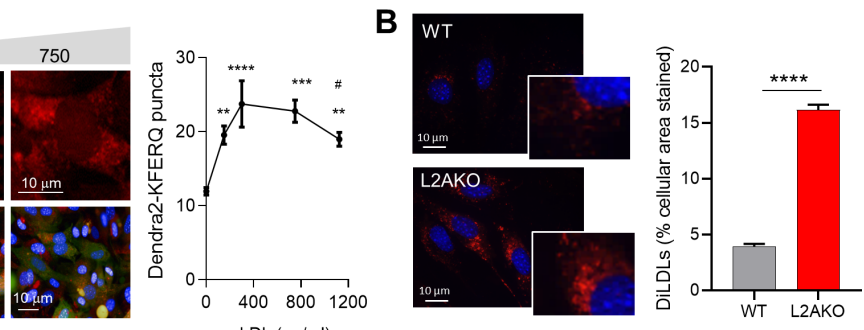

$\operatorname{LDL}(\mu \mathrm{g} / \mathrm{ml})$

D $\widehat{E}^{6} 7$ VSMC phenotypic switching

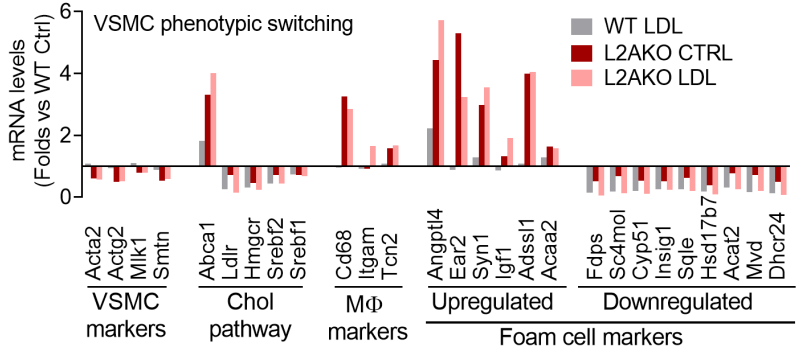

$\mathbf{F}$
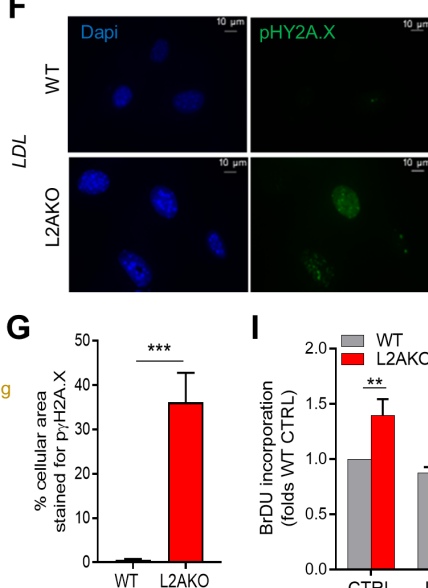

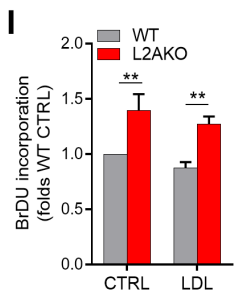

H

$$
\frac{C T R L}{W T L 2 A} \frac{L D L}{W T L 2 A}
$$

LAMP-2A

P53

P27/KIP1

P21cip1/ Waf1

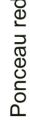

K
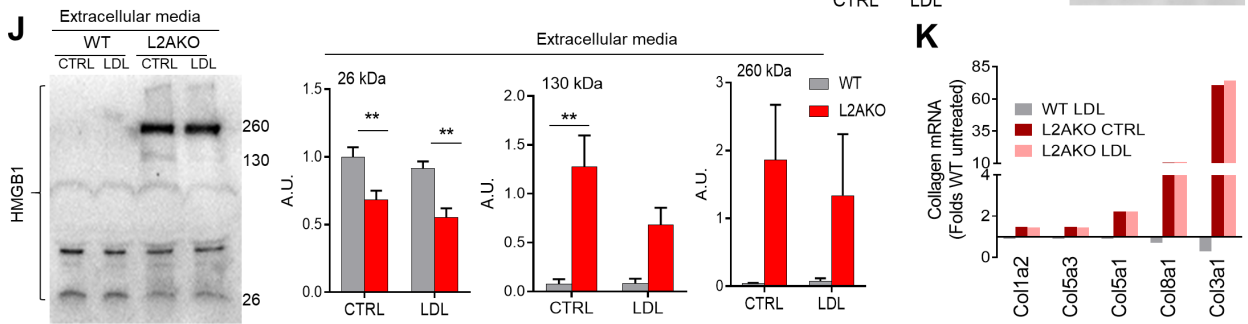

Figure 3. CMA blockage makes SMC vulnerable to lipotoxicity and promotes their dedifferentiation. A. CMA activity in SMC cells stably expressing the KFERQ-PS-Dendra2 CMA reporter and exposed to increasing concentrations of LDL. Representative images (left) of red channel (top) or merge channels (bottom). Nuclei were highlighted with DAPI. Quantification of CMA activity as the average number of fluorescent puncta per cell using high content microscopy $(n>2,500$ cells per condition in 6 different 


\section{2 | CMA in atherosclerosis}

wells and 3 independent experiments). Statistically significant differences compared with basal $\left({ }^{*}\right)$, or between groups with LDL (\#), were analyzed by one-way ANOVA with Tukey's post-hoc test ( ${ }^{* *} \mathrm{P}<$ $0.005,{ }^{* *} \mathrm{P}<0.005,{ }^{* * *} \mathrm{P}=0.001,{ }^{* * * * P}<0.001$ and $\left.\# \mathrm{P}<0.05\right)$. B. Intracellular levels of diLDL-derived fluorescence in SMC from wild type (WT) and LAMP-2A null mice (L2AKO). Representative images (left) and quantification (right) ( $n=3,>45$ cells per experiment in 3 different experiments). Inset: higher magnification. $C$. Cytotoxicity in the same cells in response to increasing concentrations of LDL (two-way ANOVA after Bonferroni's post hoc test, $F=2.862 ; P=0.9872$ for interaction; $F=2.205 ; P=$ 0.1570 for LDL concentration, $F=21.93 ; P=0.0002$ for genotype, $n=5$ )). $D$. Changes in mRNA levels of different markers of cell identity, macrophage-related and cholesterol pathway in the same SMC stimulated with LDL or maintained in a LPDS (CTRL) (pool of 3 individual experiments). E. STRING analysis for pathways differentially regulated in L2AKO cells in response to LDL compared to control (pool of 3 individual experiments). F,G. Representative images (F) and quantification (G) of immunofluorescence for pyH2A.X in WT and L2AKO primary SMC after LDL loading ( $n=3,>5$ cells per experiment). H. Immunoblot for components of the p53 signaling pathway in WT and L2AKO (L2A-/-) $\mathrm{SMC}$ in basal conditions and upon LDL loading. Ponceau red staining is shown as loading control. The experiment was repeated 4 times with similar results. I. BrDU incorporation in WT and L2AKO primary SMC in basal conditions and upon LDL loading (two-way ANOVA after Bonferroni's post hoc test, $F=0.0002639 ; P=0.0183$ for interaction; $F=19.35 ; P<0.0001$ for cells with/without $L D L, F=19.61$; $\mathrm{P}<0.0001$ for genotype, $\mathrm{n}=4-5$ ). J. Immunoblot (left) for HMGB1 in the culture media of WT and L2AKO primary SMC in basal conditions and upon LDL loading. Quantification (right) (in arbitrary densitometric units (A.D.U.) of the indicated molecular weight variants of HMGB1 (26 kDa: two-way ANOVA after Bonferroni's post hoc test, $F=0.1301 ; P=0.7246$ for interaction; $F=2.801 ; P=0.1201$ for cells with/without $L D L, F=27.50 ; P=0.0002$ for genotype; $130 \mathrm{kDa}$ : two-way ANOVA after Bonferroni's post hoc test, $F=2.578 ; P=0.1343$ for interaction; $F=2.516 ; P=0.1387$ for cells with/without LDL, $F=23.19 ; \mathrm{P}=0.0004$ for genotype; $260 \mathrm{kDa}$ : two-way ANOVA after Bonferroni's post hoc test, $F=0.2109 ; P=0.6543$ for interaction; $F=0.1635 ; P=0.6930$ for cells with/without $L D L$, $F=6.462 ; P=0.0258$ for genotype; $n=4-5)$. $K$. Changes in mRNA levels of main collagen genes in primary WT and L2AKO SMC stimulated with LDL or maintained in a LPDS (CTRL) (pool of 3 individual experiments). All data, when applicable, were tested for normal distribution using D'Agostino and Pearson normality test. Variables that did not pass normality test were subsequently analyzed using Mann-Whitney rank-sum test. All other variables were tested with the Student's t-test. Values are mean \pm SEM. $* p<0.05, * * p<0.01$ and $* * * p<0.005$.

\section{Pro-inflammatory phenotype of CMA-defective macrophages}

The presence of macrophages in the plaque and their associated inflammatory phenotype influence plaque fate. Therefore, we next set to investigate the consequences of CMA blockage in macrophage function using in vitro protocols for polarization of bone marrowderived macrophages (BMDM) to mimic the plaque pro-inflammatory phenotype of these cells (IFN $\gamma+\mathrm{LPS}$ ). We found that CMA-defective BMDM, when stimulated with IFN $\gamma+\mathrm{LPS}$, show a higher pro-inflammatory profile (higher inducible nitric oxide synthase, iNOS, and cytochrome c oxidase 2 (COX2) levels (Figure 4A-C and Figure S5A-B) suggesting that CMA may modulate pro-inflammatory polarization of macrophages. Interestingly, although the 
changes in COX2 levels were in large part due to its transcriptional upregulation, in the case of iNOS the increase was only at the level of protein, in support of changes in protein degradation contributing to the observed elevated cellular iNOS levels (Figure 4A-C).

To identify the subset of the proteome that, by not undergoing degradation through CMA, could be behind this exacerbated inflammatory phenotype seen in the L2AKO BMDM, we isolated the pool of lysosomes usually active for CMA (those that contain high levels of luminal hsc70) from WT and L2AKO BMDM, untreated (CTRL) or stimulated with IFN $\gamma+\mathrm{LPS}$. In half of the cultures, we inhibited lysosomal proteolysis to discriminate proteins undergoing degradation inside lysosomes from lysosomal resident proteins (Figure 4D and Figure $\mathbf{S 5 C}$ ), and subjected the samples to comparative quantitative proteomics ${ }^{21}$. About $45 \%$ of the proteins were constitutive lysosomal components (no degradation) both in resting and stimulated BMDM (Figure $4 \mathrm{E}$ ). CMA substrates are defined as those proteins undergoing degradation in lysosomes in a LAMP-2A-dependent manner (Figure 4D and Figure S5D-E). Stimulation with IFN $\gamma+$ LPS resulted in an increase of lysosomal protein degradation, mostly of CMA substrates (46\% increase in CMA substrates vs. only $15 \%$ increase in non-CMA lysosomal substrates; Figure 4F). In addition, the repertoire of CMA substrates degraded by untreated and stimulated BMDM was largely different, with only 7\% coinciding proteins (Figure $\mathbf{4 G}$ ). Data mining using STRING and IPA identified that the top cellular pathways associated with unstimulated macrophages were related with regulation of immune response, cell adhesion molecules and leucocyte activation, besides the expected upregulation of the pro-inflammatory LPS signaling pathway (Figure $\mathbf{4 H}$ and Figure S5F). The IFN $\gamma+$ LPS treatment induced CMA degradation of nitic oxide synthase along with five other stimulators of NO synthesis (Figure 4I), which can explain the higher levels of iNOS in CMA-incompetent macrophages upon stimulation (Figure 4A-B). CMA substrates in this condition also included proteins involved in immune response, neutrophil degradation and transendothelial migration (including cell adhesion, cellular localization and interaction with the vascular wall) (Figure $4 \mathbf{J}$ and Figure S5G). The in vivo data confirmed these findings since we found that L2AKO mice showed marked monocytosis, mainly derived from a higher number of proinflammatory monocytes (Figure $\mathbf{4 K}-\mathbf{L}$ ), and elevated number of T-cells, especially CD4+ T-cells (Figure $\mathbf{4 M - N}$ and full blood leucocyte pattern in the same mice shown in Supplemental (S) Table S2).

Overall, our findings support that CMA contributes to the remodeling of the proteome induced by macrophage stimulation, and that defective CMA in these cells promotes a more pro-inflammatory phenotype. 

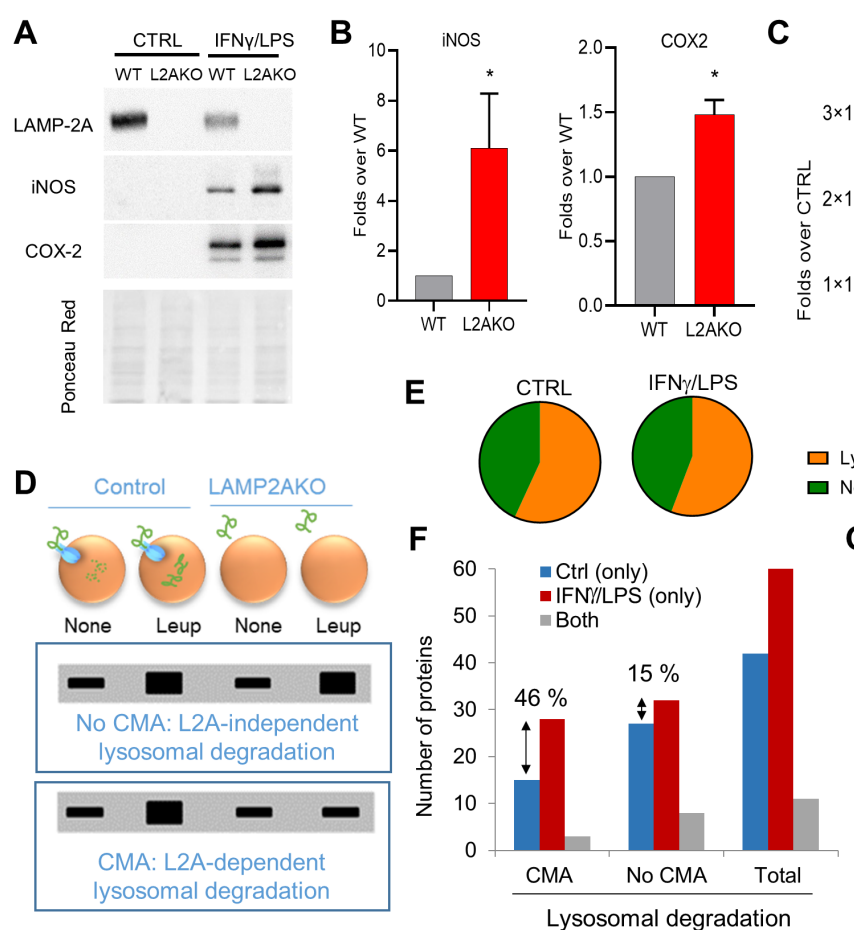

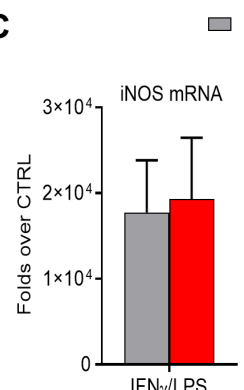

IFN $/$ LPS

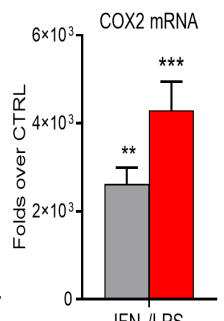

$$
\begin{aligned}
& \text { Lysosomal } \\
& \text { Non lysosomal }
\end{aligned}
$$

G

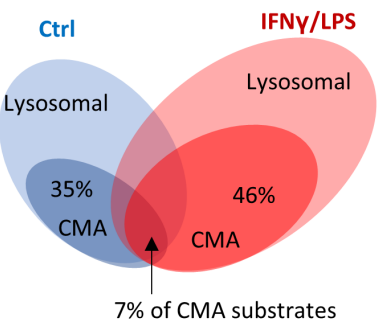

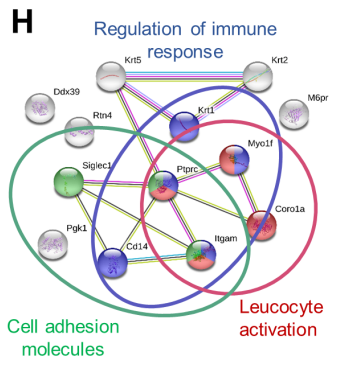

K

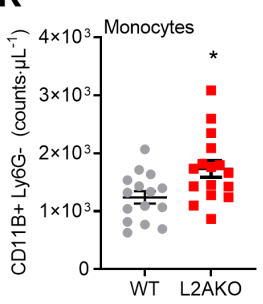

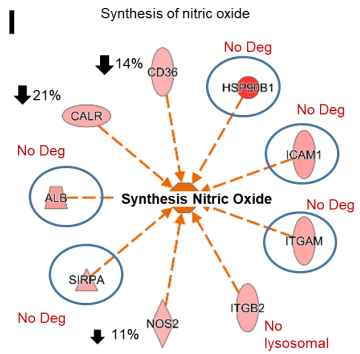

J Neutrophil degradation Immune response

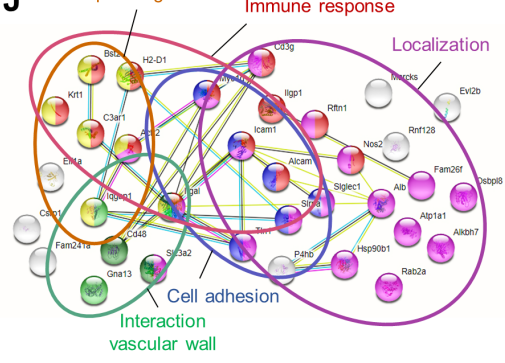

$\mathbf{L}$

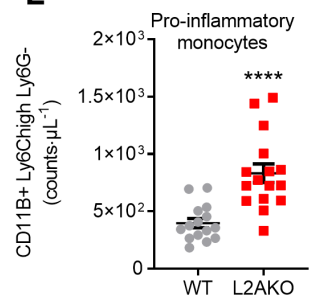

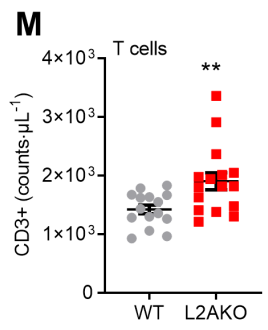

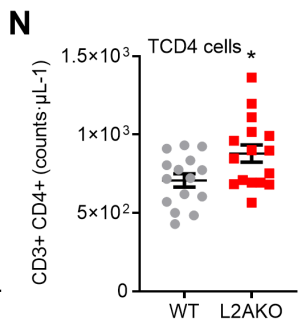

Figure 4. CMA blockage leads to exacerbated pro-inflammatory phenotype in macrophages. $A, B$. Levels of iNOS and COX-2 proteins in BMDM from wild type (WT) and LAMP-2A null mice (L2AKO) cultured without additions (control, CTRL) or stimulated with IFNY/LPS. Representative immunoblot (A) and densitometric quantification (B) expressed as folds over WT levels ( $n=5$ for iNOS and 3 for COX2). Ponceau red is shown as loading control. C. mRNA levels of iNOS and Cox 2 in the same cells 
expressed as folds over untreated (CTR). ( $n=4)$. D-J. Comparative proteomic analysis of lysosomes isolated from untreated (none) or leupeptin treated WT and L2AKO BMDM untreated (CTRL) or exposed to IFN $\gamma /$ LPS from a pool of 3 individual experiments. Schematic of the experimental design and anticipate results for hypothetical proteins undergoing CMA-dependent or -independent lysosomal degradation (D). Percentage of lysosomal (constituents) and non-lysosomal proteins (substrates) in the fractions from CTRL and IFN $\gamma /$ LPS macrophages (E). Number (F) and percentage (G) of proteins undergoing lysosomal degradation (Total) in LAMP-2A dependent (CMA) or independent (no CMA) manner. STRING analysis for top intracellular networks of CMA substrates in CTRL $(\mathbf{H})$ and IFNY/LPS (J) BMDM. Detail of changes in degradation of proteins involved in synthesis of nitric oxide (I, blue circle indicates proteins no longer degraded in lysosomes in the L2AKO group and down arrows the reduction in lysosomal degradation of those proteins in the same group). K-N. Number of total monocytes (K), pro-inflammatory subtype of monocytes (L), total T cells (M) and TCD4 cells (N) in WT and L2AKO mice ( $n=14$ WT and $n=16$ L2AKO). All data, when applicable, were tested for normal distribution using D'Agostino and Pearson normality test. Variables that did not pass normality test were subsequently analyzed using Mann-Whitney rank-sum test. All other variables were tested with the Student's t-test. All values are mean \pm SEM. Individual values are shown also in $\mathrm{i}-\mathrm{I}$. ${ }^{*} \mathrm{p}<0.05, * * \mathrm{p}$ $<0.01$ and $* * * * p<0.001$.

\section{Human carotid CMA response to pro-atherosclerotic conditions}

Our in vitro and in vivo findings support that CMA upregulation may be part of the vasculature response to pro-atherosclerotic challenges. To test whether that was also the case in human atherosclerosis, we first confirmed the presence of the CMA receptor in plaque SMC and macrophages using co-staining for LAMP-2A and $\alpha$ SMA/CD68 (Figure 5AB). Analysis of levels of LAMP-2A in human atherosclerotic plaques at different stages revealed that LAMP-2A levels at the plaque increase gradually with disease progression (graded as plaques with moderate intimal thickening (IT), pathological intimal thickening (PIT), thick fibrous cap atheroma (TkFCA) and plaques with intraplaque hemorrhage (IPH)) (Figure 5C-D). The increase in LAMP-2A protein levels originates mainly from LAMP-2A mRNA upregulation (Figure 5E). In fact, LAMP-2A mRNA levels directly correlated with the size of the plaque but not the necrotic core (Figure 5E-F). To determine the cell type (resident or/and infiltrating cells) mainly contributing to the elevated levels of LAMP-2A at the plaque, we analyzed the correlation between LAMP-2A levels and different cell types and found a direct correlation between LAMP-2A and CD68, a marker of macrophages and foam cells, in human atherosclerotic plaques (Figure 5G-H). We interpreted these changes in LAMP-2A levels as an attempt of the plaque cells, mostly macrophages, to upregulate this autophagic pathway in response to the proatherosclerotic changes prior to clinical events, as we observed in the experimental mouse model (Figure 1C).

To evaluate possible changes of CMA after the clinical event, we performed immunoblot for LAMP2 in carotid segments from patients who suffered one or two clinical vascular 


\section{2 | CMA in atherosclerosis}

events. Average of all patients revealed a significant decrease in LAMP2 levels in carotid segments from patients who develop a second event (Figure 5I). This decrease in LAMP2, seems to be driven mostly by the female patients in this group, that were the ones displaying the most pronounced changes in overall LAMP2 content (Figure $5 \mathbf{J}$ and Figure S6A-B) changes cannot be attributed to differences in patient's ages that were $66.1 \pm 3.4$ and $70.4 \pm 3.8$ for first and second event, respectively in female patients and $65.6 \pm 1.3$ and $74.3 \pm 1.2$ for the same groups in male patients). The observed changes, seem selective for LAMP2, rather than an overall reduction in the lysosomal content, since levels of cathepsin D, another lysosomal marker, remained unchanged (Figure 5I-J and Figure S6A-C).

Our findings suggest that, although CMA may increase in initial stages of human atherosclerotic disease prior to a clinical event, reduced levels of LAMP-2A, and lower CMA activity could be a predictor of the risk of suffering a second clinical event. 


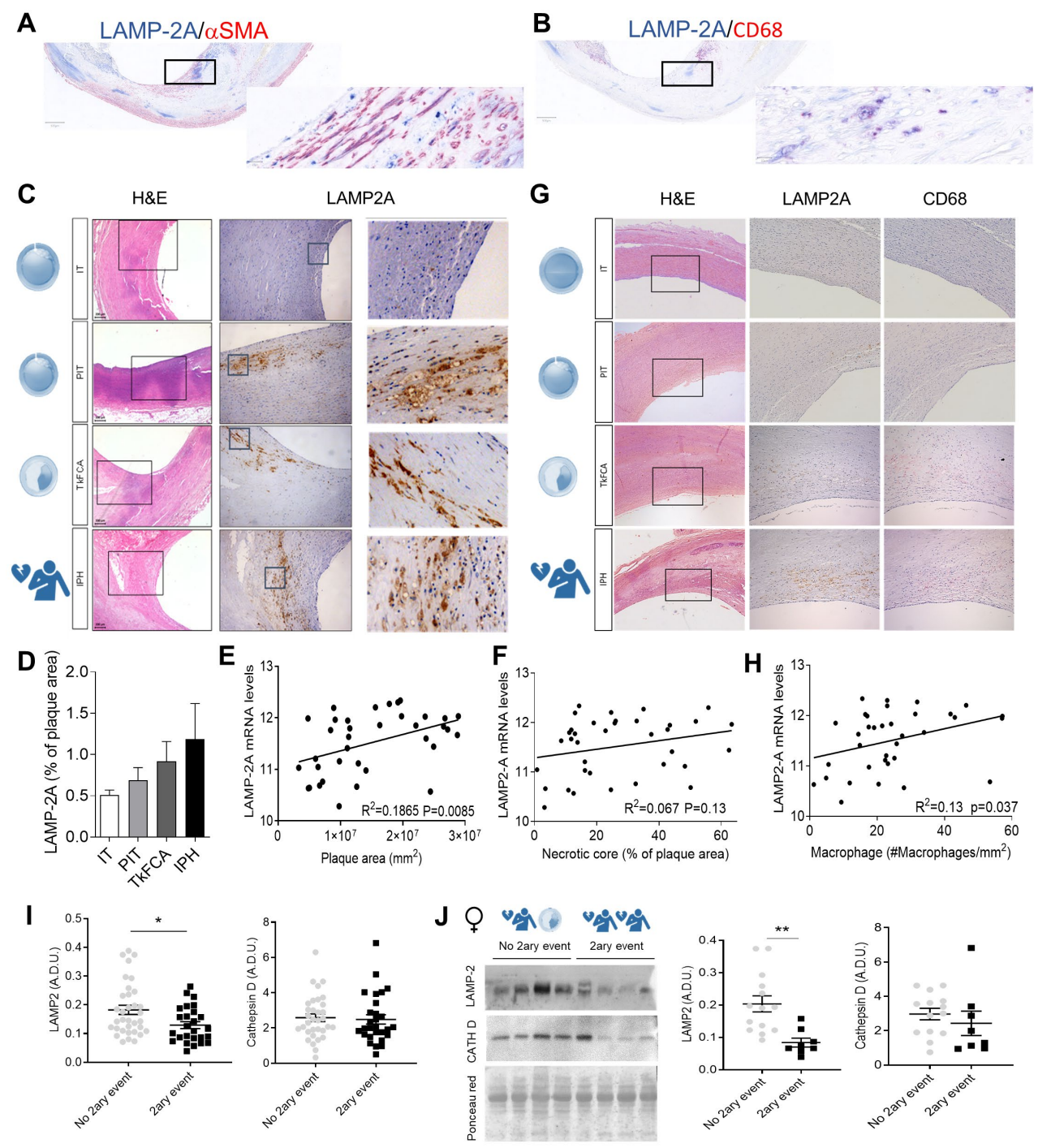

Figure 5. The CMA limiting component changes in aorta of atherosclerotic patients with disease. AG. LAMP-2A levels in different stages of atherosclerotic plaque development. Representative images of the colocalization of LAMP-2A with aSMA (A) and CD68 (B) positive cells in human atherosclerotic plaques. Representative images of H\&E staining (left) and LAMP-2A immunostaining (middle, and higher magnification on right) (C), quantification of LAMP-2A staining intensity relative to plaque area (D) and correlation between mRNA levels of LAMP-2A and plaque area (E) and extent of the necrotic core (F). Comparison of immunostaining for LAMP-2A (middle) and the macrophage marker CD68 (right) in adjacent sections from the same patient (G) and correlation between the mRNA levels of LAMP-2A and macrophage content $(\mathbf{H})$. All stains were performed in 35 human samples with an average age of 65.8 years for the patients with one event and 72.8 years for the patients with a secondary event. I-J. Protein levels for LAMP2 and Cathepsin D in plaque lysates from patients who 


\section{2 | CMA in atherosclerosis}

underwent a secondary coronary event (2ary event) or not (no 2ary event) subjected to immunoblot and electrophoresis. Average and individual values in all samples independent of gender (I) and representative immunoblot (left) and values in females only (J). Ponceau red is shown as loading control. All data, when applicable, were tested for normal distribution using D'Agostino and Pearson normality test. Variables that did not pass normality test were subsequently analyzed using MannWhitney rank-sum test. All other variables were tested with the Student's t-test. Individual patient values and mean \pm SEM are shown. ${ }^{*} \mathrm{p}<0.05,{ }^{* *} \mathrm{p}<0.01$ and ${ }^{* * *} \mathrm{p}<0.005 . \mathrm{n}=36$ for the first study. $\mathrm{n}=15-20$ for the second study.

\section{CMA upregulation protects against atherosclerosis in mice}

To experimentally test the proposed protective effect of CMA activation - observed early in the disease in mice and in human plaques - and to evaluate the possible therapeutic value of CMA modulation in atherosclerosis, we directly upregulated CMA activity in mice exposed to a pro-atherosclerotic challenge. To that end, we used an inducible transgenic mouse model ( $\mathrm{L} L 2 A O E$ ), expressing the human form of $L A M P-2 A^{33}$, which we induced after the observed drop in LAMP-2A levels (Figure 1C and Figure S7A). Although circulating cholesterol and triglyceride levels were only discretely reduced, increasing CMA activity markedly decreased the fraction of both lipids in the VLDL and LDL fractions (Figure 6A-D). As anticipated, the better lipid profile of the hL2AOE group associated with an increase in insulin sensitivity (Figure 6E). hL2AOE mice also showed a trend towards better profile of multiple pro-inflammatory cytokines (i.e. PAI1, CCL3, 4 and GCSF shown in Figure S7B,D,F) that we found modified in the opposite direction in CMAdefective mice upon the same challenge (Figure $2 \mathbf{I}$ and Figure S7C,E). Analysis of the atherosclerotic plaque revealed that hL2AOE mice exhibited smaller lesions with reduced necrotic cores and less calcification (Figure $6 \mathrm{~F}-\mathrm{I}, \mathrm{K}$ ) although collagen content, plaque stage and number of SMC and macrophages were comparable in both groups of mice (Figure 6J,L and Figure S7G-J). Principal component analysis with 12 variable data shows that $\mathrm{Ctrl}$ and hL2AOE mice groups are distributed in different regions (when using the correlation diagram with the first and second principal component) in support of these groups evolving differently upon the lipid challenge (Figure $6 \mathbf{M}-\mathbf{N}$ and Figure S7K).

Overall these findings support both systemic and vascular beneficial effects of CMA upregulation and highlight the therapeutic potential of activating CMA to prevent atherosclerotic disease progression. 


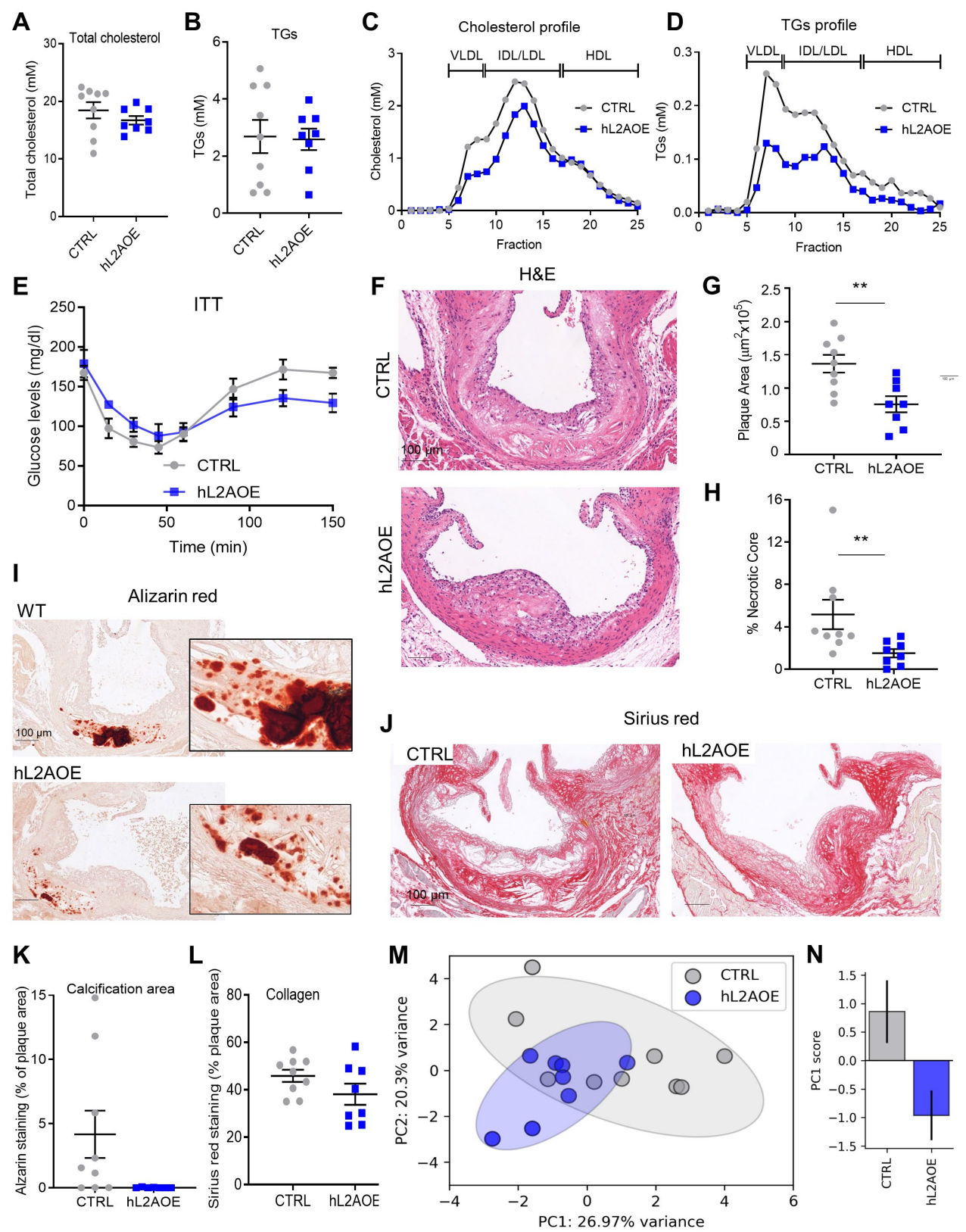

Figure 6. Genetic upregulation of CMA ameliorates disease in an atherosclerosis murine experimental model. A-D. Circulating lipids in control mice (CTRL) and in mice systemically expressing a copy of human LAMP-2A (hL2AOE) subjected to a pro-atherosclerotic intervention (injected with AAV8 PCSK9 and maintained for 12 weeks on the Western-type diet). Circulating total cholesterol (A), triglycerides (TG) (B), cholesterol profile (C) and TG profile (D). $n=9$ CTRL, $n=8$ hL2AOE. E. Insulin tolerance test in the same mice (repeated measures two-way ANOVA after Bonferroni's post hoc test, $F=3.159 ; P=0.0047$ for interaction, $F=21.02 ; P<0.0001$ for time, $F=1.578 ; P=0.098$ for genotype) 


\section{2 | CMA in atherosclerosis}

in the same mice. F-L. Properties of the plaques from aortas of the same mouse groups. Representative images of aortas stained for H\&E (F), Alizarin red (I) or sirius red (J) and quantification of plaque area $(\mathbf{G})$, size of the necrotic core $(\mathbf{H})$, calcification presence $(\mathbf{K})$ and collagen deposition $(\mathbf{L})$. $\mathrm{n}=9 \mathrm{CTRL}, \mathrm{n}=8 \mathrm{hL2AOE}$. M-N. Principal component analysis of 12 variables measured in CTRL and $\mathrm{hL2AOE}$ mice. Each dot represents a single animal (M). Ellipses are the $95 \%$ confidence interval around the center of mass of a given experimental group. Bar plot represents mean +/- s.e.m. of PC1 score for each experimental group ( $N$ ). $n=9$ CTRL, $n=8$ hL2AOE. *: Student t-test between CTRL and hL2AOE $t_{15}=2.152, p=0.048$. Individual values and mean \pm SEM are presented in all quantifications. All data were tested for normal distribution using D'Agostino and Pearson normality test. Variables that did not pass normality test were subsequently analyzed using Mann-Whitney rank-sum test. All other variables were tested with the Student's t-test. ${ }^{*} p<0.05,{ }^{*} p<0.01$ and $* * * * p<0.0001$. 


\section{Discussion}

In this work, we have identified a protective role for CMA in the defense against atherosclerotic disease through a combination of systemic and local effects (Figure 7). Fully functional CMA protects against systemic changes that promote disease progression, such as levels of circulating lipids, glucose metabolism and immuneinflammatory response. In addition, CMA in cells in the vasculature and the plaque reduces lesion severity by preserving SMC identity and modulating the macrophage proinflammatory phenotype. The aggravated atherosclerotic pathology observed upon CMA blockage in vivo underscores the contribution to disease progression of reduced CMA activity observed in aging ${ }^{28}$, diabetes ${ }^{30}$ or upon dietary lipid challenges ${ }^{25}$. We propose that upregulation of CMA is part of the organism's response to pro-atherogenic challenges, but that factors such as aging or sustained dietary pressure, known to inhibit CMA, reduce the efficacy of this protective mechanism and even predisposes to secondary clinical events in humans. We show that genetic activation of CMA slows down disease progression in mice supporting a potential therapeutic value of CMA upregulation in atherosclerosis.

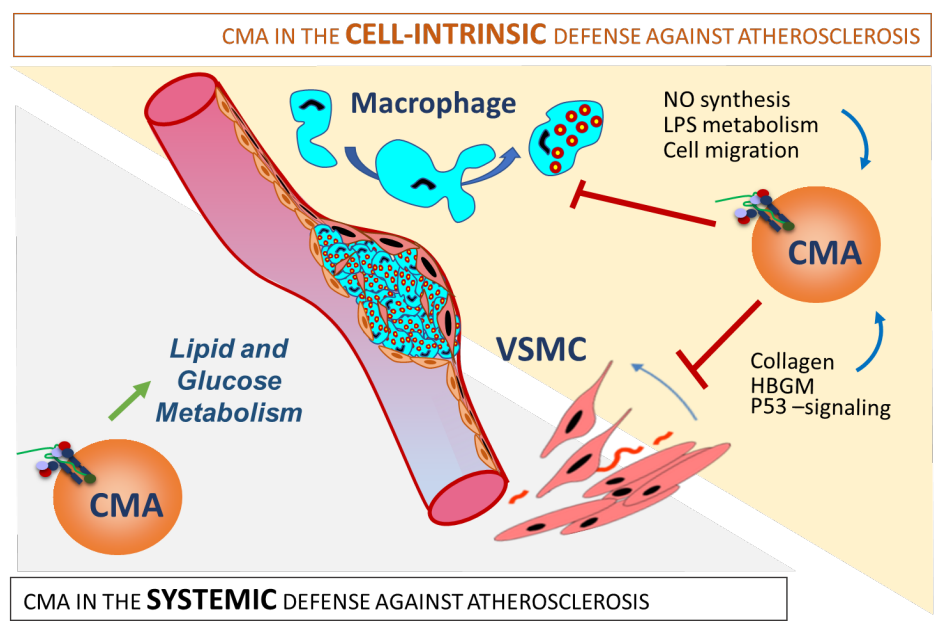

Figure 7. Schematic overview of systemic and local effects of CMA in atherosclerosis

The protective effect of CMA against atherosclerosis results from the combination of systemic and vasculature-specific functions of CMA. Left: systemic CMA failure leads to defective lipid and glucose metabolism that increases systemic vulnerability to the metabolic syndrome. Right: Defective CMA in vascular smooth cells makes them prone to dedifferentiation because of failure to degrade proteins involved in cellular proliferation, collagen secretion and cell death. Macrophages unable to upregulate CMA in response to a lipotoxic stimuli acquire a more proinflammatory phenotype with higher NO levels, increased migratory capability and defective LPS metabolism

As expected of a protein degradation pathway with the potential to degrade more than 


\section{2 | CMA in atherosclerosis}

$45 \%$ of cytosolic proteins (those with KFERQ-like motifs ${ }^{15}$ ), the mechanisms behind protective effect of CMA against atherosclerosis are multiple. Our findings support a systemic role for CMA in regulation of organismal metabolism. Blockage of CMA in liver leads to profound metabolic derangements due to failure to timely degrade proteins involved in glucose and lipid metabolism ${ }^{22}$. Defective liver metabolism in the mice with systemic CMA blockage used in this study to better mimic aging of this autophagic pathway, may be responsible for the abnormal cholesterol and lipoproteins profiles observed in these mice upon lipid challenge ${ }^{53}$. Furthermore, these mice phenocopy part of the aging metabolic phenotype characterized by diminished aerobic capacity ${ }^{54}$ and insulin resistance due to abdominal adiposity ${ }^{48}$. Several systemic changes observed in the L2AKO mice (hypercholesterolemia, hypertriglyceridemia, insulin resistance and elevated acute phase proteins such as $\mathrm{PAI}-1^{55}$ ) are major risk factors in atherosclerosis in humans and may contribute to the aggravating effect of CMA failure on the disease. We also found that part of the protective effect of CMA occurs directly at the vascular wall. CMA activity protects SMC against lipotoxicity through regulation of the cholesterol biosynthetic pathway, through combined degradation of cholesterol biogenic enzymes and of transcription factors that mediate activation of these pathways. A similar regulatory role for CMA in lipid metabolism has been described in hepatocytes through selective lysosomal degradation of lipogenic enzymes ${ }^{21}$. We also found that failure to activate CMA in response to lipid challenges is sufficient to promote a dedifferentiatedactivated like phenotype in SMC, an essential step in atherosclerosis etiology and development ${ }^{8}$. In fact, the large, fibrous and calcified plaques with extensive necrosis observed in L2AKO mice are representative of a mature stage of atherosclerosis progression that develops with aging ${ }^{56}$ ( $A H A$ classification types $V_{c}$ and $V b$, or fibrocalcific plaque according to Virmani's ${ }^{12}$ ). The enrichment of KFERQ-like motif containing proteins among the intracellular signaling factors that we found upregulated in L2AKO SMC, supports the idea that impaired timely degradation of these intracellular signaling proteins is behind the reduced viability and failure to preserve cellular identity in CMA-defective SMC. The p53 signaling pathway, the top dysfunctional pathway in L2AKO SMC, has been previously described to malfunction in cancer cells upon CMA blockage ${ }^{57}$. In SMC, reduced p53 signaling can explain the higher cellular proliferation, DNA damage and HMGB1 release and higher cell death rates observed in CMA-deficient SMC upon LDL loading.

The other cellular component of the atherosclerotic plaque directly affected by changes in CMA activity are macrophages. We found quantitative and qualitative changes in the subproteome degraded by CMA during the macrophage phenotypic switch and that defective CMA under these conditions leads to an aberrant pro-inflammatory phenotype in these cells. Among the novel CMA substrates in stimulated macrophages identified in this work, proteins involved in NO synthesis, leucocyte activation and migration, cell adhesion, neutrophil degranulation and LPS signaling have been all tightly connected 
with development of atherosclerosis ${ }^{58,59}$.

CMA is activated in response to lipotoxic stimuli, oxidative stress and hypoxia ${ }^{25-27}{ }_{L}$ all coexisting conditions at the atherosclerotic plaque ${ }^{3,60,61}$. We propose that the high levels of LAMP-2A identified with human plaque progression in asymptomatic subjects may be indicative of reactive CMA upregulation (as the one observed in mice in the early stages of the pro-atherosclerotic challenge) to protect against later events in the human disease. In fact, we found a significant (albeit gender-dependent) decrease in LAMP2 abundance compatible with reduced CMA activity in patients with a second event, when compared with those who only had one event. Although it is not possible to determine the cause of this reduced LAMP2 content, comparative lipidomic analysis in mice chronically exposed to lipid challenges demonstrated that sustained changes in the lipid composition of the lysosomal membrane promote rapid degradation of LAMP-2A in this compartment ${ }^{25}$.

Our genetic intervention to upregulate CMA activity by overexpressing hLAMP-2A in vivo, proved to be successful to slow down atherosclerotic disease progression in mice. Although future studies on the status of CMA at the different disease stages in patients are needed to determine the possible timeframe of the efficacy of this intervention in humans, our work provides proof of concept for the therapeutic value of CMA upregulation in atherosclerosis. Interestingly, CMA upregulation has a more profound effect on metabolic function compared to its direct effect on the local plaque environment. Thus, hL2AOE mice exposed to the pro-atherosclerotic challenge have better overall metabolic profile, whereas locally occurring events, such as collagen content or number of SMC and macrophages at the plaque were comparable to control mice. Nevertheless, the fact that plaque size and necrotic core were significantly smaller when CMA was upregulated, make CMA a very attractive therapeutic target for atherosclerosis.

\section{Acknowledgements}

We thank Dr. Jose Javier Bravo-Cordero (Mt. Sinai) for his assistance with the acquisition of images from aorta of KFERQ-PS-Dendra2 mice, Trea Streefland (LUMC) for her assistance with generating the lipid profiles and Dr. Susmita Kaushik (Einstein) for critical reading of the manuscript and useful feedback. This work was supported by National Institutes of Health grants AG021904, AG031782 and DK098408 (to AMC), the A15CVD04 Leducq Foundation Network (to AMC and JCS), and a Dr. Dekker senior postdoc fellowship from the Dutch Heart Foundation (2016T060 to JCS). JMM was supported by a postdoctoral fellowship from the American Heart Association 17POST33650088.

\section{Authors Contributions}

JMM designed and performed biochemical and image-based experiments, analyzed and 


\section{2 | CMA in atherosclerosis}

interpreted the data, wrote the first draft and edited later versions of the manuscript; JdB, KVK, BS and MJJG performed histological staining and analysis of human and mouse arteries, analyzed and interpreted the data and contributed to editing of the manuscript; ST assisted with sample collection and biochemical analysis; $A D$ assisted with animal genotyping, diets and maintenance; IT generated and systemically characterized the LAMP-2A OE mice; EALB and JCS coordinated the Paired Segment of Carotid Endarterectomy samples "MaasHPS" Biobank; AMS assisted with sample collection; MB performed the computational analysis; $\mathrm{HJ}$ analyzed human plaque microarray data; PR andJB performed the cholesterol and TG profile; GP collected the prospective AtheroExpress Study Biobank with human carotid plaques; AMC and JCS coordinated the study, coordinated the autopsy-derived human plaque Biobank, contributed to designing and interpretation of the experiments and to editing the manuscript. All authors read and edited the final draft of the manuscript.

\section{Competing Financial Interest}

AMC consults for Neuropore (La Joya, CA, USA) and is cofounder of Selphagy Therapeutics (Boston, MA, USA).

\section{Data availability}

There are not restrictions on data availability in this manuscript. No database was generated but microarray data was deposited in GEO accession number GSE49553. Source Data for main and Supplemental Figure figures and for Table 1 are provided as individual Excel worksheet for each figure (Source Data file). 


\section{References}

1. Benjamin EJ, Blaha MJ, Chiuve SE, et al. Heart Disease and Stroke Statistics-2017 Update: A Report From the American Heart Association. Circulation. 2017;135:e146-e603. 2. Libby P. Vascular biology of atherosclerosis: overview and state of the art. Am J Cardiol. 2003;91:3A-6A.

3. Tabas I, Williams KJ and Boren J. Subendothelial lipoprotein retention as the initiating process in atherosclerosis: update and therapeutic implications. Circulation. 2007;116:1832-44.

4. Moore KJ and Tabas I. Macrophages in the pathogenesis of atherosclerosis. Cell. 2011;145:341-55.

5. Robbins CS, Hilgendorf I, Weber GF, et al. Local proliferation dominates lesional macrophage accumulation in atherosclerosis. Nat Med. 2013;19:1166-72.

6. Jonasson L, Holm J, Skalli O, et al. Regional accumulations of T cells, macrophages, and smooth muscle cells in the human atherosclerotic plaque. Arteriosclerosis. 1986;6:1318.

7. Vengrenyuk $\mathrm{Y}$, Nishi $\mathrm{H}$, Long $\mathrm{X}$, et al. Cholesterol loading reprograms the microRNA143/145-myocardin axis to convert aortic smooth muscle cells to a dysfunctional macrophage-like phenotype. Arterioscler Thromb Vasc Biol. 2015;35:535-46.

8. Bennett MR, Sinha $S$ and Owens GK. Vascular Smooth Muscle Cells in Atherosclerosis. Circ Res. 2016;118:692-702.

9. Wirka RC, Wagh D, Paik DT, et al. Atheroprotective roles of smooth muscle cell phenotypic modulation and the TCF21 disease gene as revealed by single-cell analysis. Nature Medicine. 2019;25:1280-+.

10. Mitchinson MJ, Hardwick SJ and Bennett MR. Cell death in atherosclerotic plaques. Curr Opin Lipidol. 1996;7:324-9.

11. Proudfoot D, Skepper JN, Hegyi L, et al. Apoptosis regulates human vascular calcification in vitro: evidence for initiation of vascular calcification by apoptotic bodies. Circ Res. 2000;87:1055-62.

12. Virmani R, Kolodgie FD, Burke AP, et al. Lessons from sudden coronary death: a comprehensive morphological classification scheme for atherosclerotic lesions. Arterioscler Thromb Vasc Biol. 2000;20:1262-75.

13. Mizushima N and Komatsu M. Autophagy: renovation of cells and tissues. Cell. 2011;147:728-41.

14. Dice JF. Peptide sequences that target cytosolic proteins for lysosomal proteolysis. Trends Biochem Sci. 1990;15:305-309.

15. Kirchner P, Bourdenx M, Madrigal-Matute J, et al. Proteome-wide analysis of chaperone-mediated autophagy targeting motifs. PLoS Biol. 2019;17:e3000301.

16. Chiang $\mathrm{H}$, Terlecky S, Plant $\mathrm{C}$, et al. A role for a 70-kilodalton heat shock protein in lysosomal degradation of intracellular proteins. Science. 1989;246:382-385. 


\section{2 | CMA in atherosclerosis}

17. Cuervo AM and Dice JF. A receptor for the selective uptake and degradation of proteins by lysosomes. Science. 1996;273:501-503.

18. Bandyopadhyay U, Kaushik S, Varticovski L, et al. The chaperone-mediated autophagy receptor organizes in dynamic protein complexes at the lysosomal membrane. Mol Cell Biol. 2008;28:5747-63.

19. Agarraberes FA, Terlecky SR and Dice JF. An intralysosomal hsp70 is required for a selective pathway of lysosomal protein degradation. J Cell Biol. 1997;137:825-34.

20. Salvador N, Aguado C, Horst M, et al. Import of a cytosolic protein into lysosomes by chaperone-mediated autophagy depends on its folding state. J Biol Chem. 2000;275:27447-56.

21. Schneider JL, Suh Y and Cuervo AM. Deficient chaperone-mediated autophagy in liver leads to metabolic dysregulation. Cell Metab. 2014;20:417-32.

22. Kaushik S and Cuervo AM. Degradation of lipid droplet-associated proteins by chaperone-mediated autophagy facilitates lipolysis. Nature cell biology. 2015;17:759-70.

23. Valdor R, Mocholi E, Botbol $\mathrm{Y}$, et al. Chaperone-mediated autophagy regulates $\mathrm{T}$ cell responses through targeted degradation of negative regulators of $\mathrm{T}$ cell activation. Nature immunology. 2014;15:1046-54.

24. Cuervo AM, Hildebrand H, Bomhard EM, et al. Direct lysosomal uptake of alpha 2microglobulin contributes to chemically induced nephropathy. Kidney Int. 1999;55:529-45.

25. Rodriguez-Navarro JA, Kaushik S, Koga H, et al. Inhibitory effect of dietary lipids on chaperone-mediated autophagy. Proceedings of the National Academy of Sciences of the United States of America. 2012;109:E705-14.

26. Kiffin R, Christian C, Knecht E, et al. Activation of chaperone-mediated autophagy during oxidative stress. Molecular biology of the cell. 2004;15:4829-40.

27. Dohi E, Tanaka S, Seki T, et al. Hypoxic stress activates chaperone-mediated autophagy and modulates neuronal cell survival. Neurochem Int. 2012;60:431-42.

28. Cuervo AM and Dice JF. Age-related decline in chaperone-mediated autophagy. J Biol Chem. 2000;275:31505-13.

29. Kiffin $R$, Kaushik S, Zeng $M$, et al. Altered dynamics of the lysosomal receptor for chaperone-mediated autophagy with age. J Cell Sci. 2007;120:782-91.

30. Sooparb S, Price SR, Shaoguang J, et al. Suppression of chaperone-mediated autophagy in the renal cortex during acute diabetes mellitus. Kidney Int. 2004;65:2135-44.

31. Madrigal-Matute J, Scorrano L and Sadoshima J. Leducq Network: Modulating Autophagy to Treat Cardiovascular Disease. Circ Res. 2018;123:323-325.

32. Dong S, Aguirre-Hernandez C, Scrivo A, et al. Monitoring spatiotemporal changes in chaperone-mediated autophagy in vivo. Nat Commun. 2020;11:645.

33. Tasset I, Diaz A, Dong S, et al. Anti-aging effects of systemic restoration of chaperone-mediated autophagy. (in preparation).

34. Bjorklund MM, Hollensen AK, Hagensen MK, et al. Induction of atherosclerosis in mice and hamsters without germline genetic engineering. Circ Res. 2014;114:1684-9. 
35. Huh WJ, Khurana SS, Geahlen JH, et al. Tamoxifen induces rapid, reversible atrophy, and metaplasia in mouse stomach. Gastroenterology. 2012;142:21-24 e7.

36. Araldi E, Fernandez-Fuertes M, Canfran-Duque A, et al. Lanosterol Modulates TLR4Mediated Innate Immune Responses in Macrophages. Cell Rep. 2017;19:2743-2755.

37. Argmann CA, Sawyez CG, Li S, et al. Human smooth muscle cell subpopulations differentially accumulate cholesteryl ester when exposed to native and oxidized lipoproteins. Arterioscler Thromb Vasc Biol. 2004;24:1290-6.

38. Canfran-Duque A, Rotllan N, Zhang $X$, et al. Macrophage deficiency of miR-21 promotes apoptosis, plaque necrosis, and vascular inflammation during atherogenesis. EMBO Mol Med. 2017;9:1244-1262.

39. Martinez FO, Gordon S, Locati M, et al. Transcriptional profiling of the human monocyte-to-macrophage differentiation and polarization: new molecules and patterns of gene expression. J Immunol. 2006;177:7303-11.

40. Sluimer JC, Kisters N, Cleutjens KB, et al. Dead or alive: gene expression profiles of advanced atherosclerotic plaques from autopsy and surgery. Physiol Genomics. 2007;30:335-41.

41. Olmeda D, Cerezo-Wallis D, Riveiro-Falkenbach E, et al. Whole-body imaging of lymphovascular niches identifies pre-metastatic roles of midkine. Nature. 2017;546:676680.

42. Koga $H$, Martinez-Vicente $M$, Macian F, et al. A photoconvertible fluorescent reporter to track chaperone-mediated autophagy. Nat Commun. 2011;2:386.

43. Arias E, Koga H, Diaz A, et al. Lysosomal mTORC2/PHLPP1/Akt Regulate ChaperoneMediated Autophagy. Mol Cell. 2015;59:270-84.

44. Storrie B and Madden EA. Isolation of subcellular organelles. Methods Enzymol. 1990;182:203-25.

45. Madrigal-Matute J, Fernandez-Garcia CE, Gomez-Guerrero C, et al. HSP90 inhibition by 17-DMAG attenuates oxidative stress in experimental atherosclerosis. Cardiovasc Res. 2012;95:116-23.

46. Kimura S, Noda T and Yoshimori T. Dissection of the autophagosome maturation process by a novel reporter protein, tandem fluorescent-tagged LC3. Autophagy. 2007;3:452-60.

47. Schneider JL, Villarroya J, Diaz-Carretero A, et al. Loss of hepatic chaperonemediated autophagy accelerates proteostasis failure in aging. Aging Cell. 2015;14:249-64.

48. Kohrt WM, Kirwan JP, Staten MA, et al. Insulin resistance in aging is related to abdominal obesity. Diabetes. 1993;42:273-81.

49. Zheng JP, Ju D, Jiang $\mathrm{H}$, et al. Resveratrol induces $\mathrm{p} 53$ and suppresses myocardinmediated vascular smooth muscle cell differentiation. Toxicol Lett. 2010;199:115-22.

50. Davalos AR, Kawahara M, Malhotra GK, et al. p53-dependent release of Alarmin HMGB1 is a central mediator of senescent phenotypes. J Cell Biol. 2013;201:613-29. 


\section{2 | CMA in atherosclerosis}

51. Stros M, Muselikova-Polanska E, Pospisilova S, et al. High-affinity binding of tumorsuppressor protein p53 and HMGB1 to hemicatenated DNA loops. Biochemistry. 2004;43:7215-25.

52. Parra $M$, Jardi $M$, Koziczak $M$, et al. p53 Phosphorylation at serine 15 is required for transcriptional induction of the plasminogen activator inhibitor-1 (PAl-1) gene by the alkylating agent N-methyl-N'-nitro-N-nitrosoguanidine. J Biol Chem. 2001;276:36303-10.

53. Goldstein JL and Brown MS. A century of cholesterol and coronaries: from plaques to genes to statins. Cell. 2015;161:161-172.

54. Fleg JL, Morrell CH, Bos AG, et al. Accelerated longitudinal decline of aerobic capacity in healthy older adults. Circulation. 2005;112:674-82.

55. Festa A, D'Agostino R, Jr., Mykkanen L, et al. Relative contribution of insulin and its precursors to fibrinogen and PAI-1 in a large population with different states of glucose tolerance. The Insulin Resistance Atherosclerosis Study (IRAS). Arterioscler Thromb Vasc Biol. 1999;19:562-8.

56. van Lammeren GW, Reichmann BL, Moll FL, et al. Atherosclerotic plaque vulnerability as an explanation for the increased risk of stroke in elderly undergoing carotid artery stenting. Stroke. 2011;42:2550-5.

57. Kon $\mathrm{M}$, Kiffin $\mathrm{R}$, Koga $\mathrm{H}$, et al. Chaperone-mediated autophagy is required for tumor growth. Science translational medicine. 2011;3:109ra117.

58. Burleigh ME, Babaev VR, Oates JA, et al. Cyclooxygenase-2 promotes early atherosclerotic lesion formation in LDL receptor-deficient mice. Circulation. 2002;105:181623.

59. Detmers PA, Hernandez M, Mudgett J, et al. Deficiency in inducible nitric oxide synthase results in reduced atherosclerosis in apolipoprotein E-deficient mice. J Immunol. 2000;165:3430-5.

60. Martin-Ventura JL, Madrigal-Matute J, Martinez-Pinna R, et al. Erythrocytes, leukocytes and platelets as a source of oxidative stress in chronic vascular diseases: detoxifying mechanisms and potential therapeutic options. Thromb Haemost. 2012;108:435-42.

61. Sluimer JC, Gasc JM, van Wanroij JL, et al. Hypoxia, hypoxia-inducible transcription factor, and macrophages in human atherosclerotic plaques are correlated with intraplaque angiogenesis. J Am Coll Cardiol. 2008;51:1258-65. 


\section{Supplemental data}

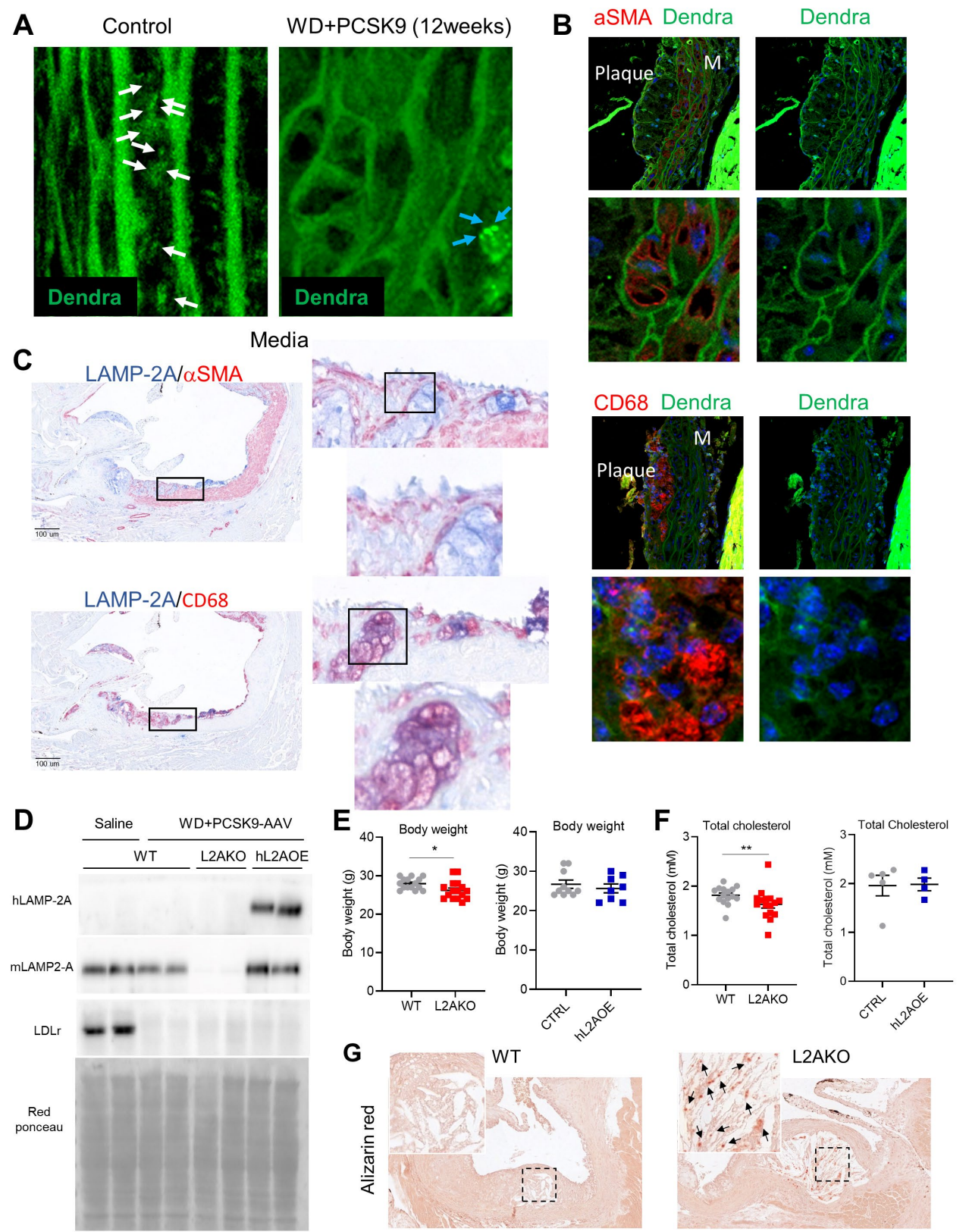

Supplemental Figure S1. Characterization of the mouse models used in this study. A-B. Representative images of aortas from KFERQ-Dendra2 mice untreated (Control) or subjected to a proatherosclerotic treatment (injected with AAV8 PCSK9 and maintained for 12 weeks on the Westerntype diet. Higher magnification images (A) of media and plaque shown in Figure 1a to better appreciate the puncta (white arrows). Blue arrows show fluorescent puncta in a cell outside the 


\section{2 | CMA in atherosclerosis}

plaque as control of the CMA read out. Images of individual and merged channels (B) of the insets shown in Figure $1 b$ of aorta sections co-stained with $\alpha$-SMA or CD68 to better appreciate absence of green fluorescent puncta in cells at the plaque. Experiments in panels $a$ and $b$, were repeated 3 times with similar results. C. Representative images of the colocalization of LAMP-2A with $\alpha$ SMA (top) and CD68 positive cells (bottom) in a murine atherosclerotic plaque. D. Immunoblot for human LAMP-2A (hLAMP-2A), murine LAMP-2A (mLAMP-2A) and LDLR in livers from wild type (WT), LAMP-2A null mice (L2AKO) and mice systemically expressing a copy of human LAMP-2A (hL2AOE) injected retro-orbitally with saline or AAV8-PCSK9 as indicated. Ponceau red is shown as loading control. E-F. Basal metabolic parameters in WT, L2AKO and hL2AOE mice at 3 months of age. Body weight (E) and total cholesterol levels (F). ( $n=15$ CTR, $n=16$ L2AKO, $n=9$ hL2AOE). G. Representative images of Alizarin red staining of aorta from WT and L2AKO mice subjected to the pro-atherosclerotic intervention. Insets: boxed area at higher magnification. Arrows: calcium deposits. Quantification is shown in Figure 10. All data, when applicable, were tested for normal distribution using D'Agostino and Pearson normality test. Variables that did not pass normality test were subsequently analyzed using Mann-Whitney rank-sum test. All other variables were tested with the Student's t-test. Individual values (symbols) and mean \pm SEM are shown. ${ }^{*} p<0.05$ and ${ }^{* *} p<0.01$. 
A

\begin{tabular}{|c|c|c|c|}
\cline { 2 - 4 } \multicolumn{1}{c|}{} & WT & L2AKO & p-value \\
\hline Liver $(\mathrm{mg} / \mathrm{mm})$ & $6.62 \pm 0.19$ & $9.69 \pm 0.47$ & **** \\
\hline eWAT $(\mathrm{mg} / \mathrm{mm})$ & $3.66 \pm 0.31$ & $6.79 \pm 0.75$ & $* * *$ \\
\hline Kidneys $(\mathrm{mg} / \mathrm{mm})$ & $1.81 \pm 0.05$ & $1.78 \pm 0.1$ & ns \\
\hline Feces (g/day) & $0.23 \pm 0.016$ & $0.19 \pm 0.011$ & $*$ \\
\hline
\end{tabular}

C

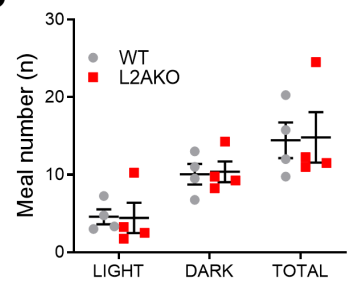

E

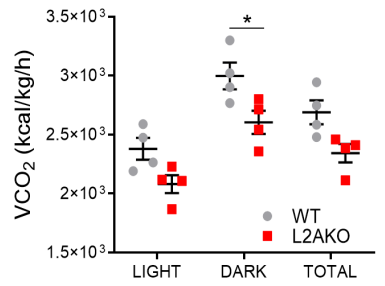

B

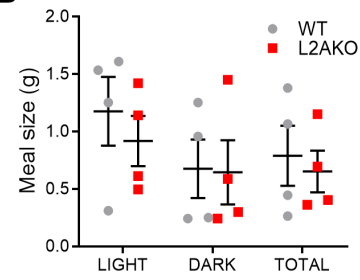

D

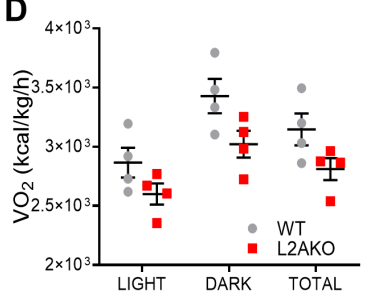

$\mathbf{F}$

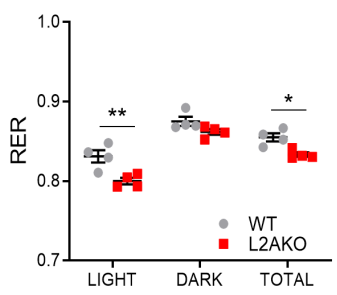

Supplemental Figure S2. Metabolic profile of CMA deficient mice subjected to an experimental model of murine atherosclerosis. A. Tissue weights after 12 weeks of Western-type diet $(n=15)$ in wild-type (WT) and LAMP-2A null mice (L2AKO). Tissue weights are normalized by tibial length and expressed as mean \pm SEM. B-F. Metabolic parameters measured by indirect calorimetry in WT and L2AKO mice fed a Western-type diet for 6 weeks: Meal size (Meal size: two-way ANOVA, $F=0.1027 ; P=0.9029 \quad$ for interaction, $F=1.359 ; P=0.2820$ for light/dark/total, $F=0.4767 ; P=0.4987$ for genotype, $n=4)(B)$, meal number (Meal number: two-way ANOVA, $F=0.01 ; P=0.99$ for interaction, $F=12.83 ; P=0.0003$ for light $/$ dark/total, $F=0.0122 ; P=$ 0.9133 for genotype, $n=4)(C)$, volume of $\mathrm{O}_{2}$ consumption ambulatory parameters (VO2: two-way ANOVA, $F=0.1759 ; P=0.8401$ for interaction, $F=8.587 ; \mathrm{P}=0.0024$ for light/dark/total, $F=12.04 ; \mathrm{P}=$ 0.0027 for genotype, $\mathrm{n}=4$ ) (D), volume of $\mathrm{CO}_{2}$ production (VCO2: two-way ANOVA, $F=0.1254 ; P=0.8829$ for interaction, $F=18.38 ; P<0.0001$ for light/dark/total, $F=20.33 ; P=$ 0.0003 for genotype, $n=4$ ) $(E)$ and RER (RER: two-way ANOVA, $F=1.537 ; P=0.2419$ for interaction, $F=54.04 ; P<0.0001$ for light/dark/ total, $F=27.8 ; P<0.0001$ for genotype, $n=4$ ) (F). All data were tested for normal distribution using D'Agostino and Pearson normality test. Variables that did not pass normality test were subsequently analyzed using Mann-Whitney rank-sum test. All other variables were tested with the Student's t-test. ns indicates $p$-value $=$ non-significant. Graphs represent individual values, mean \pm SEM. ${ }^{*} p<0.05,{ }^{* *} p<0.01$ and ${ }^{* * *} p<0.005$. 
A

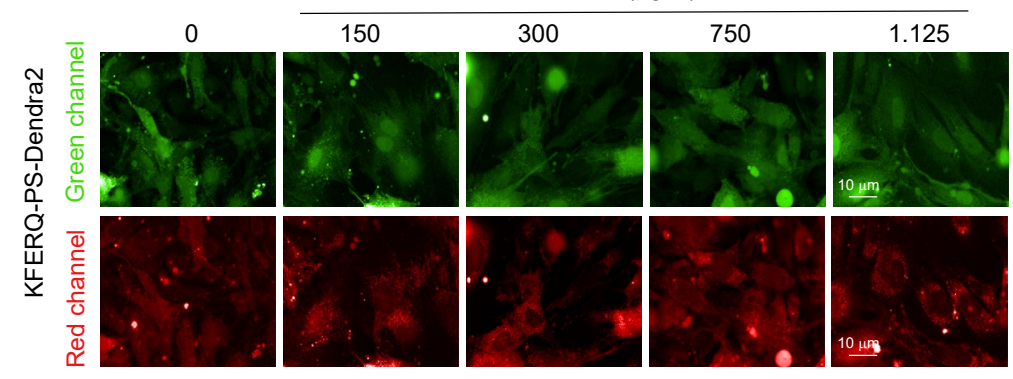

B
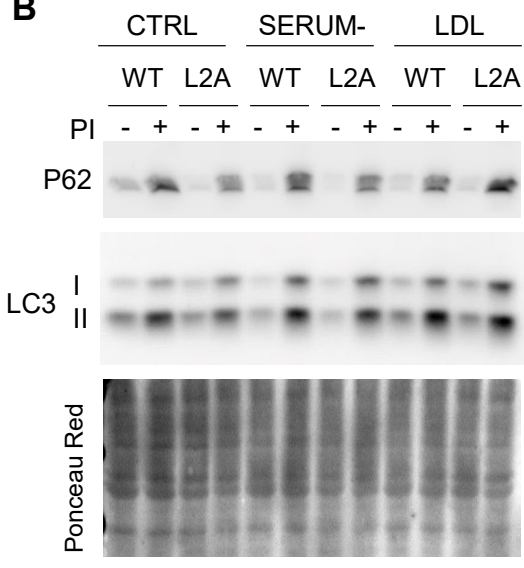

c mCherry-GFP-LC3
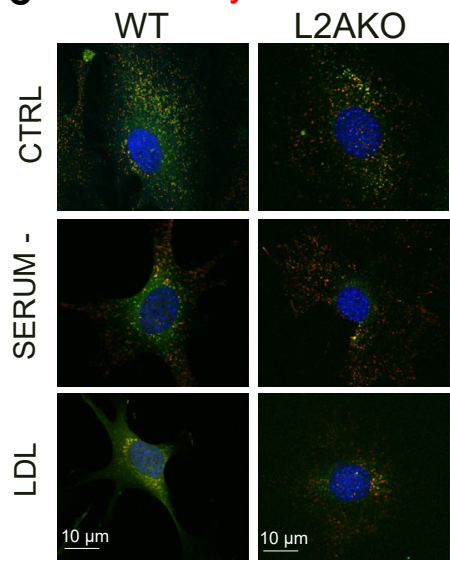

Supplemental Figure S3. Autophagic changes in SMC upon lipid challenges. A. CMA activity in murine SMC stably expressing the KFERQ-PS-Dendra2 CMA reporter and challenged with the indicated concentrations of LDL. Representative images of separate channels. Quantification is shown in Figure 3a. B. Macroautophagy flux detected by immunoblot for LC3-II and P62/SQSTM1 in WT and L2AKO SMC under the indicated conditions, detected as an increase in LC3-II and p62 intensity upon inhibition of lysosomal hydrolysis with protease inhibitors (PI). Ponceau red was used as loading control. The experiment was repeated 4 times with similar results. C. Macroautophagy flux detected by direct fluorescence in WT and L2AKO SMC transfected with the tandem reporter mCherry-GFP-LC3. Representative images of merged channels are shown. Nuclei are highlighted with DAPI. The experiment was repeated 4 times with similar results. 


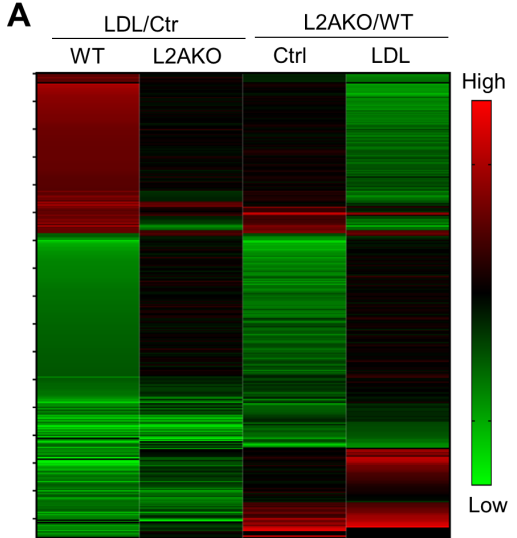

B

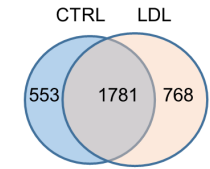

Genes up- or down-regulated in L2AKO

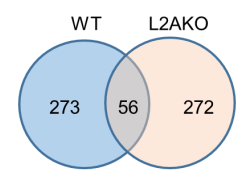

Genes up- or down-regulated in response to lipid loading
C

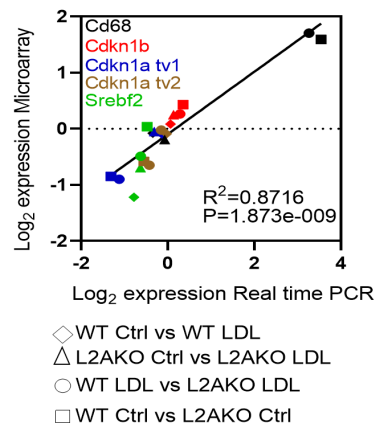

F WT in response to LDL
D

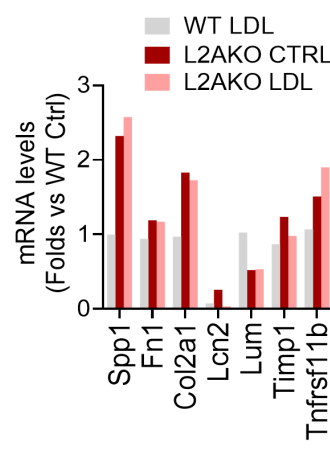

G

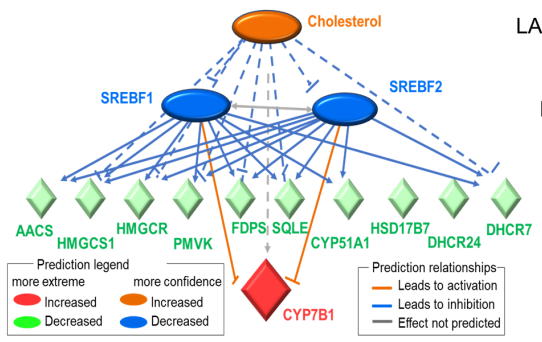

$\mathbf{E}$

Up in L2AKO CTRL vs WT CTRL

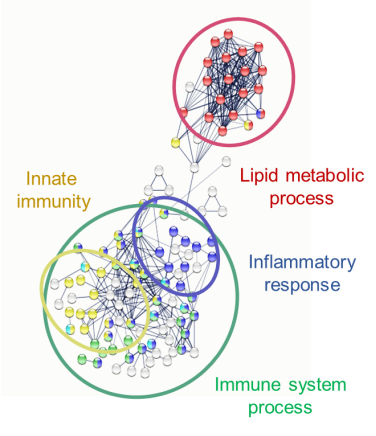

L2AKO in response to LDL

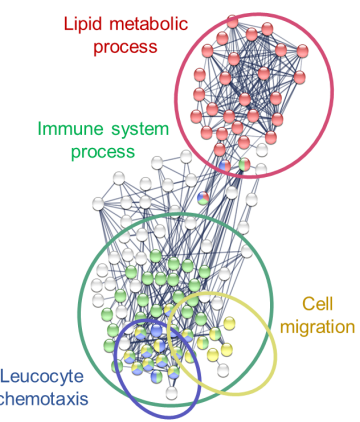

Supplemental Figure S4. Differential transcriptional and protein profile of CMA-deficient SMC. A. Hierarchical heatmap of transcriptional changes (Log2 fold) in SMC from wild type (WT) and LAMP-2A null mice (L2AKO) cultured under basal conditions (control, CTRL) or upon LDL loading. B. Venn diagrams showing the number of genes upregulated and downregulated (cutoff $> \pm 1 \log _{2}$ fold change) WT and L2AKO SMC in basal conditions (top) or in response to LDL treatment (bottom) compared to the same conditions in the WT cells. C. Validation by RT-PCR of selected genes identified in the microarray in three independent experiments and to Hprt1 expression. Scatter plot showing the significant positive relationship between selected mRNA gene expression obtained by microarray and 


\section{2 | CMA in atherosclerosis}

RT-PCR. Level of significance was determined by Pearson correlation. $R^{2}=0.8716 ; P=1.873 e-09$. D. Changes in mRNA levels of genes recently identified as markers of modified dedifferentiated SMC ${ }^{9}$ in primary WT and L2AKO SMC stimulated with LDL or maintained in a LPDS (CTRL) (pool of 3 individual experiments). E-F. STRING analysis for pathways upregulated in L2AKO cells under basal conditions (E) or changing in response to LDL loading (F) in WT (top) or L2AKO SMC (bottom). G. Cholesterol biosynthetic network identified as one of the top networks of genes modulated differentially in WT and L2AKO primary SMC. Red: increase, green: decrease. $\mathbf{H}$. Representative immunoblot for HMGB1 in cellular lysates of WT and L2AKO primary SMC maintained in basal conditions or upon serum removal (-) or LDL loading. The experiment was repeated 3 times. 
A

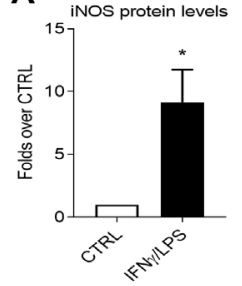

B

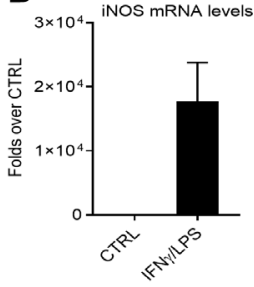

C

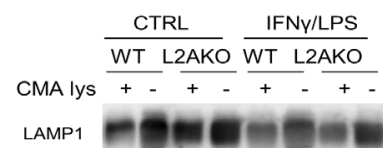

LAMP-2A $=-\mathrm{E}=$
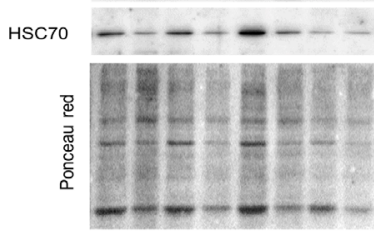

$\mathbf{F}$

G
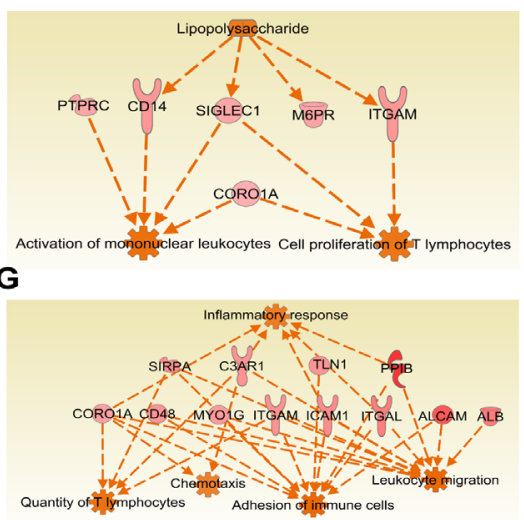

D
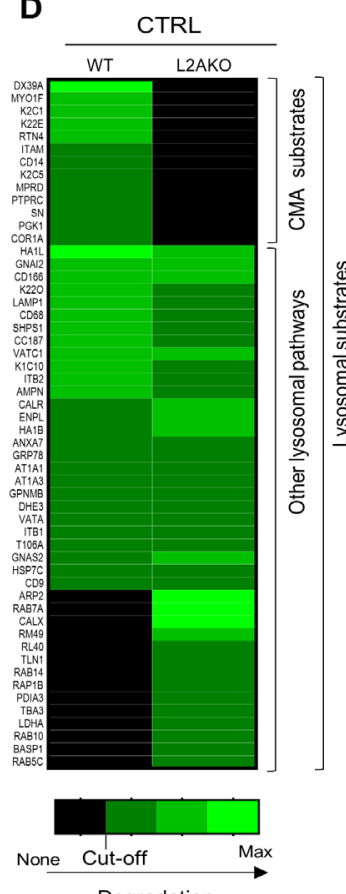

Degradation

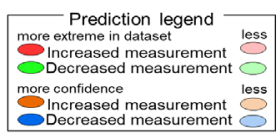

Prediction relationships - Leads to activation - Leads to inhibition - Effect not predicted

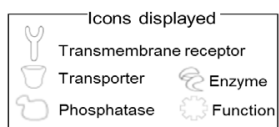

$\mathbf{E}$
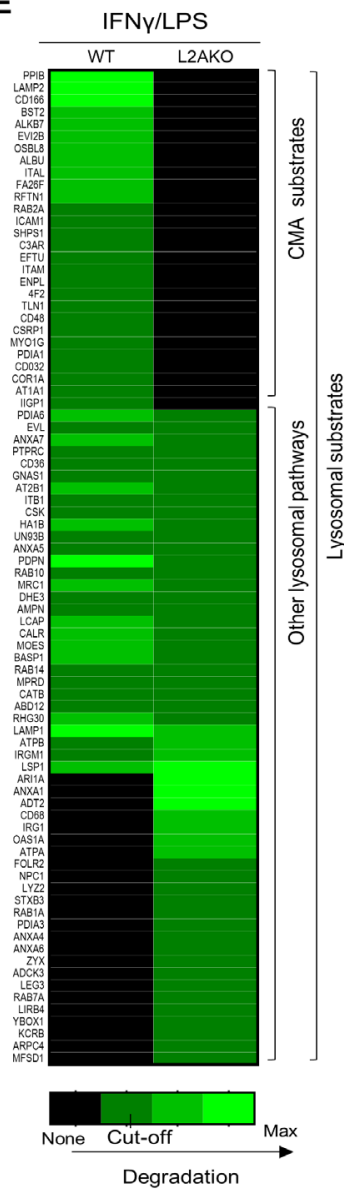

Supplemental Figure S5. Characterization of CMA-deficient macrophages. A-B. Levels of iNOS protein (A) and mRNA (B) in BMDM from wild type (WT) and LAMP-2A null mice (L2AKO) cultured without additions (control, CTRL) or stimulated with IFN $\gamma / L P S . ~ n=4$. C. Representative immunoblot for the indicated lysosomal components (LAMP1, LAMP-2A and HSC70) in lysosomes with high (+) or low $(-)$ activity for CMA isolated from WT or L2AKO mouse BMDM. Ponceau red is shown as loading control. The experiment was repeated 3 times with similar results. D-E. Heat map of changes in levels of the proteome of lysosomes from WT or L2AKO mouse BMDM CTRL (D) or exposed to IFNY/LPS (E) upon inhibition of lysosomal proteolysis. F-G. Predicted activation in BMDM L2AKO cells of the LPS pathway due to accumulation of CMA substrates (F) and the inflammatory response in IFN $/$ /LPS treated cells (G) using the IPA software. All data, when applicable, were tested for normal distribution using D'Agostino and Pearson normality test. Variables that did not pass normality test were subsequently analyzed using Mann-Whitney rank-sum test. All other variables were tested with the Student's t-test. Graphs represent mean \pm SEM. ${ }^{*} p<0.05,{ }^{* *} p<0.01$ and ${ }^{* * * *} p<0.0001$. 

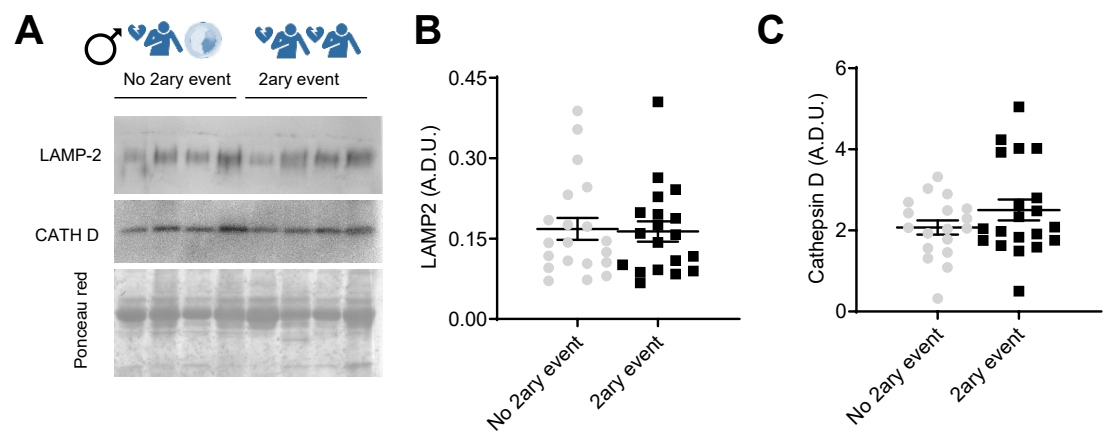

Supplemental Figure S6. Changes in CMA components in carotid from atherosclerosis patients. Protein levels for LAMP2 and Cathepsin D in plaque lysates from male patients who underwent a secondary coronary event (2ary event) or not (no 2ary event) subjected to immunoblot. Representative immunoblot (A) and individual and mean values of the densitometric quantifications for LAMP2 (B) and cathepsin D (C) are shown as arbitrary densitometric units (A.D.U.). Ponceau red is shown as loading control. All data, when applicable, were tested for normal distribution using D'Agostino and Pearson normality test. Variables that did not pass normality test were subsequently analyzed using Mann-Whitney rank-sum test. All other variables were tested with the Student's ttest. Individual patient values and mean \pm SEM are shown. $n=36$. 

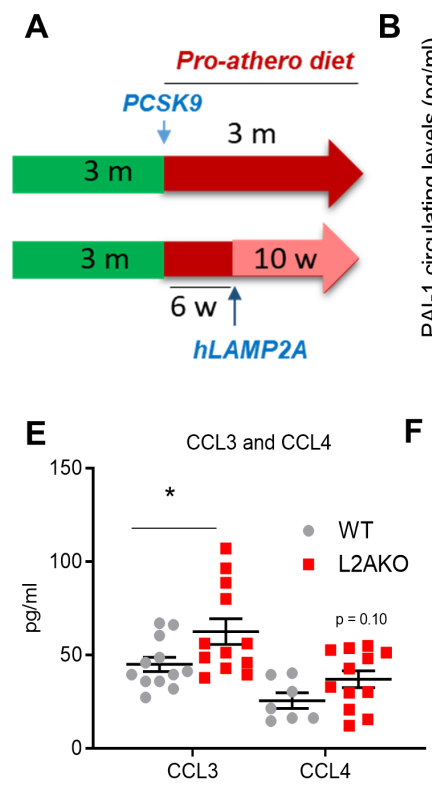

H
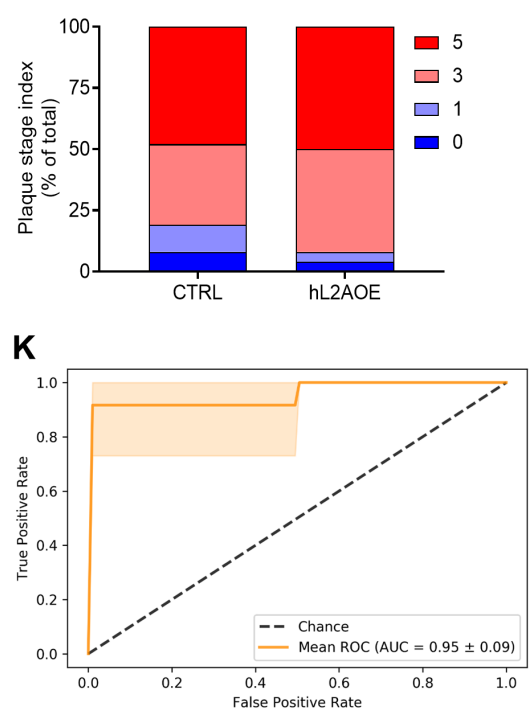
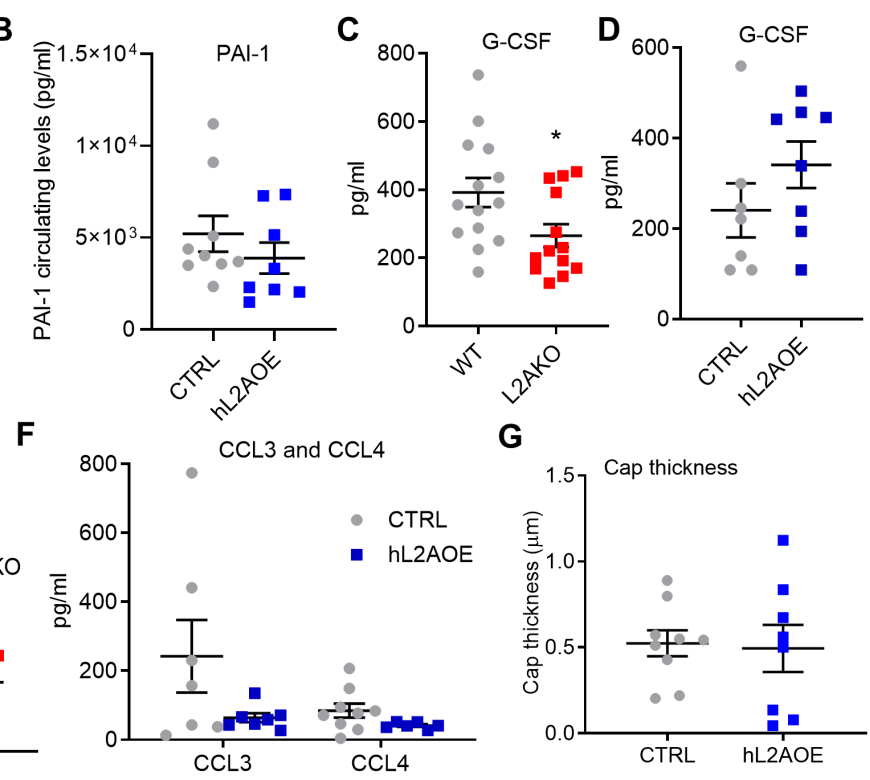

G

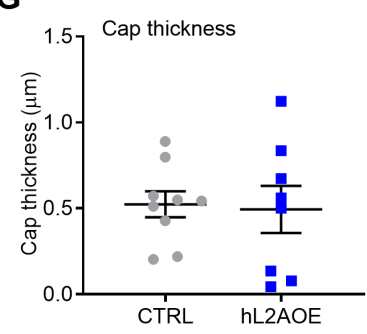

I
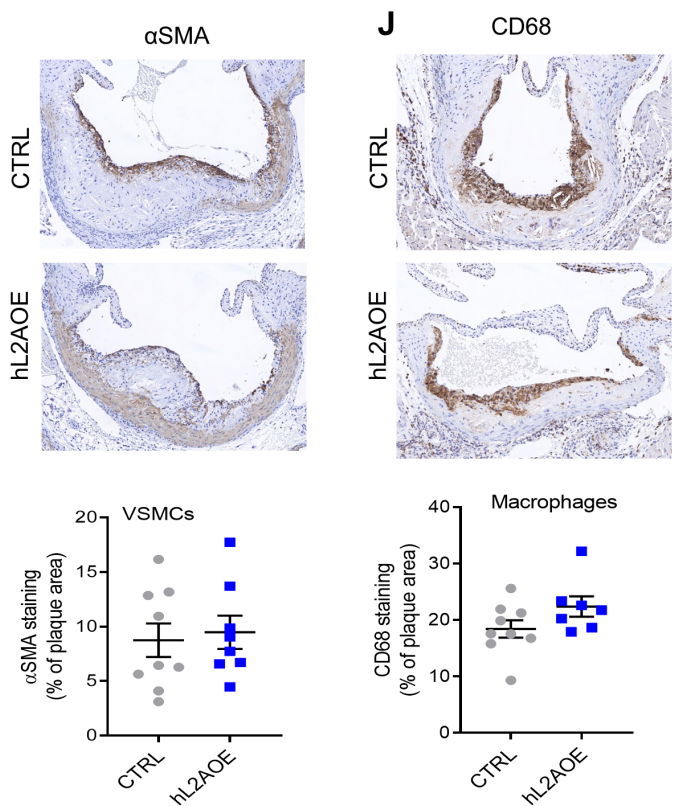

Supplemental Figure S7. Characterization of mice with genetic upregulation of CMA subjected to an experimental model of murine atherosclerosis. a. Scheme of the intervention to induce atherosclerosis (pro-athero) in mice systemically expressing a copy of human LAMP-2A (hL2AOE). b-f. Levels of circulating PAI-1(b), G-CSF (c,d) and CCL3 and CCL4 $(e, f)$ in control $(n=9)$ and hL2AOE $(n=8)$ mice (b,d,f) and in wild-type mice (WT, $n=15$ ) and mice systemically null for LAMP-2A (L2AKO, n=16) (c,e). g-j. Properties of the plaques from aortas of CTR and hL2AOE mice subjected to the pro- 


\section{2 | CMA in atherosclerosis}

atherosclerotic intervention. Quantification of cap thickness $(\mathbf{g})$, plaque stage $(\mathbf{h})$ and representative images (top) and quantification (bottom) of aortas immunostained for $\alpha S M A+S M C$ (i) and CD68 macrophages (j) Individual values (symbols) and mean \pm SEM are shown. $n=9$ CTRL, $n=8$ hL2AOE. k. Receiver operating characteristic curve showing the performance of a support vector machine with linear kernel trained to classify animals between CTRL and $\mathrm{hL2AOE}$ groups. Orange line represents the mean performance over 5 folds cross validation. AUC: area under the curve, ROC: receiver operating characteristics. All data, when applicable, were tested for normal distribution using D'Agostino and Pearson normality test. Variables that did not pass normality test were subsequently analyzed using Mann-Whitney rank-sum test. All other variables were tested with the Student's t-test. Graphs represent mean \pm SEM. ${ }^{*} p<0.05,{ }^{* *} p<0.01$ and ${ }^{* * * *} p<0.0001$. 
Supplemental Table 1. List of primers for murine samples used in this study.

\begin{tabular}{|c|c|c|}
\hline Gene & Forward primer & Reverse primer \\
\hline $\begin{array}{l}\text { Cd68 } \\
\text { (tv 1) }\end{array}$ & GGACCGCTTATAGCCCAAGG & GGATGGCAGGAGAGTAACGG \\
\hline $\begin{array}{l}\text { Cdkn1a } \\
\text { (tv 1) }\end{array}$ & CGGTGTCAGAGTCTAGGGGA & GCCTGTGGCTCTGAATGTCT \\
\hline $\begin{array}{l}\text { Cdkn1a } \\
\text { (tv 2) }\end{array}$ & TGGAGACAGAGACCCCAGAT & CAGGATTGGACATGGTGCCT \\
\hline Cdkn1b & CAGACGTAAACAGCTCCGAATTA & ACACAGGTAGTACAACAAAGCAA \\
\hline $\begin{array}{l}\text { Ptgs2 } \\
\text { (Cox2) }\end{array}$ & CTTCGGGAGCACAACAGAGT & AAGTGGTAACCGCTCAGGTG \\
\hline $\begin{array}{l}\text { Lamp-2 } \\
\text { (Lamp- } \\
\text { 2a,tv 1) }\end{array}$ & CTTAGCTTCTGGGATGCCCC & GCACTGCAGTCTTGAGCTGT \\
\hline $\begin{array}{l}\text { Nos2 } \\
\text { (iNOS) }\end{array}$ & TCCTGGACATTACGACCCCT & AGGCCTCCAATCTCTGCCTA \\
\hline Srebf2 & GTCTCCCTGAGCTGGACCT & TAGCATCTCGTCGATGTCCC \\
\hline
\end{tabular}

Tv: transcript variant. 
2 | CMA in atherosclerosis

Supplemental Table 2. Blood leukocyte count

\begin{tabular}{|c|c|c|c|}
\hline & WT & L2AKO & p-value \\
\hline CD45+ leucocytes & $9013 \pm 378$ & $9825 \pm 620$ & ns \\
\hline B220+ B cells & $3825 \pm 196$ & $3273 \pm 2931$ & ns \\
\hline NK.1.1+ NK cells & $193 \pm 24$ & $160 \pm 10$ & ns \\
\hline NK.1.1 low CD3low NK T cells & $25.7 \pm 3.9$ & $21.5 \pm 2.1$ & ns \\
\hline $\begin{array}{l}\text { NK.1.1 low CD3low CD4+ NKT } \\
\text { CD4 cells }\end{array}$ & $3.43 \pm 0.68$ & $2.91 \pm 0.31$ & ns \\
\hline $\begin{array}{c}\text { NK.1.1 low CD3low CD8+ NKT } \\
\text { CD8 cells }\end{array}$ & $5.28 \pm 0.78$ & $5.84 \pm 0.48$ & ns \\
\hline NK.1.1- CD3- Non NK T cells & $7250 \pm 327$ & $7596 \pm 474$ & ns \\
\hline CD11B+ Ly6G+ Granulocytes & $1238 \pm 84$ & $1705 \pm 113$ & $* * *$ \\
\hline CD11B+ Ly6C- Monocytes & $1237 \pm 107$ & $1729 \pm 144$ & * \\
\hline $\begin{array}{c}\text { CD11B+ Ly6C- Low } \\
\text { inflammatory monocytes }\end{array}$ & $680 \pm 70$ & $714 \pm 61$ & ns \\
\hline $\begin{array}{c}\text { CD11B+ Ly6C Med Monocytes } \\
\text { mid-inflammatory }\end{array}$ & $152 \pm 11$ & $174 \pm 15$ & ns \\
\hline $\begin{array}{l}\text { CD11B+ Ly6C High } \\
\text { inflammatory monocytes }\end{array}$ & $396 \pm 40$ & $832 \pm 81$ & $* * * *$ \\
\hline CD3+ Total T cells & $1421 \pm 75$ & $1902 \pm 145$ & $* *$ \\
\hline CD3+ CD4+ TCD4 cells & $707 \pm 43$ & $879 \pm 55$ & $*$ \\
\hline CD3+ CD8+ TCD8 cells & $617 \pm 35$ & $760 \pm 46$ & * \\
\hline $\begin{array}{c}\text { CD3+ CD8+ Ly6C high Tmem } \\
\text { cells }\end{array}$ & $302 \pm 22$ & $336 \pm 21$ & ns \\
\hline
\end{tabular}

Blood leukocyte count in wild type (WT) mice and mice systemically knocked out for LAMP-2A (L2AKO) maintained on a Western-type diet ( $n=15$ per genotype). All data are expressed as counts/ $\mu \mathrm{l}$ and were tested for normal distribution using D'Agostino and Pearson normality test. Variables that did not pass normality test were subsequently analyzed using Mann-Whitney rank-sum test. All other variables were tested with the Student's t-test. Graphs represent mean \pm SEM. ${ }^{*} p<0.05,{ }^{* *} p<0.01, * * * p$ $<0.005$ and $* * * * \mathrm{p}<0.0001$. ns $=$ nonsignificant. 
CMA in atherosclerosis | $\mathbf{2}$ 



\section{Chapter 3}

\section{The hypoxia-sensor carbonic anhydrase IX affects macrophage metabolism, but is not a suitable biomarker for human cardiovascular disease}

Demandt JAF, Dubois LJ, van Kuijk K, Zatovičová M, Jin H, Parkkila S, van der Laan SW, Jelenska L, Mees BME, Reutelingsperger CPM, Cleutjens KBJM, van der Kallen CJH, Schalkwijk CG, van Greevenbroek MMJ, Biessen EAL, Pasterkamp G, Pastoreková S, Stehouwer CDA, Sluimer JC

Sci Rep. 2021 Jan 11;11(1):425 



\section{Abstract}

Hypoxia is prevalent in atherosclerotic plaques, promoting plaque aggravation and subsequent cardiovascular disease (CVD). Transmembrane protein carbonic anhydrase IX (CAIX) is hypoxia-induced and can be shed into circulation as soluble CAIX (sCAIX). As plaque macrophages are hypoxic, we hypothesized a role for CAIX in macrophage function, and as biomarker of hypoxic plaque burden and CVD. As tumor patients with probable CVD are treated with CAIX inhibitors, this study will shed light on their safety profile. CAIX colocalized with macrophages (CD68) and hypoxia (pimonidazole), and correlated with lipid core size and pro-inflammatory iNOS+ macrophages in unstable human carotid artery plaques. Although elevated $\mathrm{pH}$ and reduced lactate levels in culture medium of CAIX knockout (CAIXKO) macrophages confirmed its role as $\mathrm{pH}$-regulator, only spare respiratory capacity of CAIXKO macrophages was reduced. Proliferation, apoptosis, lipid uptake and expression of pro- and anti-inflammatory genes were not altered. Plasma sCAIX levels and plaque-resident CAIX were below the detection threshold in 50 and $90 \%$ of asymptomatic and symptomatic cases, respectively, while detectable levels did not associate with primary or secondary events, or intraplaque hemorrhage. Initial findings show that CAIX deficiency interferes with macrophage metabolism. Despite a correlation with inflammatory macrophages, plaque-resident and SCAIX expression levels are too low to serve as biomarkers of future CVD. 


\section{3 | CAIX in atherosclerosis}

\section{Introduction}

Atherosclerotic plaque development and subsequent rupture are pivotal in the development of clinical cardiovascular disease (CVD), and orchestrated by close interplay between inflammation and cholesterol. Abundant plaque macrophages require vast amounts of oxygen, which is scantily present in the plaque core $\mathrm{e}^{1-3}$. Macrophage hypoxia is thought to make plaques prone to intraplaque hemorrhage (IPH) and rupture, subsequently leading to clinical thrombotic events. Plaque hypoxia imaging with $\left[{ }^{18} \mathrm{~F}\right]-\mathrm{HX} 4$ could identify human plaques with traits associated to rupture, ${ }^{2}$ hence plasma biomarkers of plaque hypoxia may offer a cost-effective alternative to identify patients with rupture-prone atherosclerotic plaques.

To this end, carbonic anhydrase IX (CAIX) could be a suitable biomarker, as it is a hypoxiainduced transmembrane protein of which the extracellular $4 \mathrm{kDa}$ component can be shed into body fluids by a disintegrin and metalloprotease (ADAM) $17 .{ }^{4}$ Under physiological conditions, it is mainly expressed in stomach and proximal intestinal epithelial cells. ${ }^{5,6} \mathrm{CAIX}$ function and the biomarker potential of soluble CAIX (SCAIX) to asses tumor hypoxia have been widely studied in the context of cancer. ${ }^{7-10}$ In short, cancer cells adapt to glycolysis as their main source of ATP, regardless of oxygen availability, known as the Warburg effect. ${ }^{11}$ This results in pericellular acidification, promoting cancer cell proliferation, migration and invasion. ${ }^{12}, 13$ Moreover, the metabolically adapted cancer cells become resistant to radiation and chemotherapy induced cell death. ${ }^{14}$ CAIX plays an important role in this transition to and maintenance of the Warburg effect and subsequent extracellular acidification, serving as $\mathrm{pH}$ sensor and regulator of intra- and extracellular $\mathrm{pH}^{15}$ In fact, inhibiting CAIX function was shown to potentiate radiation and chemotherapy in multiple types of cancer, underlining the importance of CAIX in tumor cell survival. ${ }^{9}$

How do these findings of the cancer field translate to macrophage function in the atherosclerotic plaque? First of all, development of plaque hypoxia might induce expression of CAIX and subsequently increase circulating levels of SCAIX. As plaque hypoxia is associated with plaque hemorrhage and instability, ${ }^{1-3}$ plaque-resident CAIX or SCAIX could thus be associated with plaque instability. Secondly, the Warburg effect has also been described in activated macrophages, ${ }^{16}$ and hypoxic macrophages will thus rely on glycolysis for fuel supply. ${ }^{17}$ Furthermore, in advanced human carotid plaques, $\mathrm{pH}$ was shown to be as low as $6.8,{ }^{18}$ possibly due to CAIX activity, while physiologic interstitial $\mathrm{pH}$ is $\sim 7.4$. Low $\mathrm{pH}$ is known to have detrimental effects on atherogenesis. In RAW267.7 macrophages, low pH was sufficient to induce a pro-inflammatory macrophage phenotype. ${ }^{19}$ In addition, low $\mathrm{pH}$ will also increase pericellular matrix degradation, alter lipid homeostasis and hamper macrophage lipid handling. ${ }^{20}$ Furthermore, several pro-atherogenic lipid modifications are induced by intraplaque acidification, potentially promoting plaque progression. Indeed, deficiency of another $\mathrm{pH}$ regulator, $\mathrm{Na}+\mathrm{H}+$ exchanger 1 , led to reduced plaque formation. ${ }^{21}$ Altogether this corroborates the hypothesis that plaque acidification, possibly via 
macrophage CAIX, could promote plaque development and excessive expression of this protein might identify advanced plaques with a hypoxic, and/or acidic milieu. As therapeutics directed against CAIX are pursued in cancer patients, ${ }^{9}$ an elderly group with a likely burden of CVD, the role of CAIX in atherosclerosis should be investigated to assess the CVD safety profile. In addition, CAIX and SCAIX could be biomarkers of plaque hypoxia and/or acidity to predict future cardiovascular events. Hence, in this study, we aimed to explore the expression of CAIX in human atherosclerosis, a role for CAIX in atherogenic macrophage functions and its potential as a biomarker for atherosclerotic disease. 


\section{3 | CAIX in atherosclerosis}

\section{Methods}

\section{Animal tissues}

Femur and tibia were obtained from adult CAIXKO and WT mice on a C57/BI6 background from breeding colonies in Oulu University, Finland to culture bone-marrow-derived macrophages. Use of adult CAIXko and WT mice for bone marrow isolation was in concordance with FELASA recommendations and was approved by the Animal Experimentation Committee of the University of Oulu. The CA IX deficient mice have been described and characterized earlier. ${ }^{22,23}$ No live animals were used in this study.

\section{Cell culture}

Bone marrow was isolated and cells were cultured for seven days in RPMI-1640 (Gibco with Glutamax, 2g/L glucose) supplemented with $10 \%$ FCS, 100U/ml Penicillin-Streptomycin, and 15\% L929-conditioned medium to generate BMDMs. BMDMs were always allowed to attach for 24 hours, prior to any additional stimuli. BMDMs were polarized for 24 hours to proinflammatory M1 macrophages (LPS $(10 \mathrm{ng} / \mathrm{ml})$ and IFN $\gamma(100 \mathrm{u} / \mathrm{ml})$ ), to anti-inflammatory M2A (IL-4, 20ng/ml) or M2C (IL-10, 10ng/ml) macrophages or to lipid laden Mox macrophages (oxLDL, $25 \mu \mathrm{g} / \mathrm{ml}$ ).

\section{Apoptosis}

BMDMs were stimulated with 50 $\mathrm{M}$ 7-ketocholesterol (Sigma, C2394) for 24 hours to induce apoptosis. After stimulation, nuclei were stained with Hoechst (15 $\mu \mathrm{g} / \mathrm{ml}$, Sigma) and apoptotic cells with fluorescently labeled AnxA5-FP488 (produced by Biochemistry department, Maastricht University) for 15 minutes. Samples were analyzed using a highthroughput, fluorescent reporter system, coupled to automated microscopy (BD Pathway 855 High Content Bioimager). Data was processed with Attovision and BD Diva software.

\section{Lipid uptake}

Isolation of LDL and subsequent oxidation into oxLDL is described elsewhere. ${ }^{24}$ Post-dialysis oxLDL concentration was determined using the bicinchoninic acid (BCA) protein assay kit (Pierce, 23227). Cells were either put in hypoxic $\left(1 \% \mathrm{O}_{2}\right)$ or normoxic culture conditions for 24 hours. BMDMs were incubated for 3 hours with a mix oxLDL (8ug/ml) and Topfluor cholesterol (Avanti Polar Lipids, 810255). After wash, nuclei were stained using Hoechst $(15 \mu \mathrm{g} / \mathrm{ml}$, Sigma Aldrich). Samples were analyzed using a high-throughput, fluorescent reporter system, coupled to automated microscopy (BD Pathway 855 High Content Bioimager). Data was processed with Attovision and BD Diva software. 


\section{EdU incorporation}

Cells were exposed to hypoxia $\left(1 \% \mathrm{O}_{2}\right)$ or normoxia for 24 hours. Afterwards, EdU (5ethynyl-2'-deoxyuridine) (Thermo Fisher Scientific, A10044) was added (10 $\mu \mathrm{M})$ for 2 hours. Cells were fixed ( $3.7 \%$ formaldehyde in PBS) and permeabilized ( $0.1 \%$ Triton ${ }^{\circledR} \mathrm{X}-100$ in PBS). Subsequently, Click-iT reaction mix was added (1x Click-iT cell reaction buffer, cell buffer additive, CuSO4 (Thermo Fisher Scientific, C10269), and Alexa-fluor 594 azide (2.5 $\mu \mathrm{M})$ (Thermo Fisher Scientific, A10270)). Nuclei were stained with hoechst $(15 \mu \mathrm{g} / \mathrm{ml}$, Sigma Aldrich). Samples were analyzed using a high-throughput, fluorescent reporter system, coupled to automated microscopy (BD Pathway 855 High Content Bioimager). Data was processed with Attovision and BD Diva software.

\section{Proliferation}

Proliferation of BMDMs was measured on an ACEA xCELLigence (Roche). Unstimulated BMDMs ( $8 \times 10^{4}$ cells) were seeded on a gold electrode implemented in a 96 wells plate and allowed to grow for 72 hours. Impedance was measured hourly and used to quantify proliferation (slope of impedance increment over time) using RCTA software (version 1.2, Roche).

\section{Seahorse mitochondrial test}

Cells were plated in optimal seeding density ( 40.000 cells/well) in an XFe96 cell culture microplate (Agilent, 102416-100) and allowed to attach for 24 hours under standard culture conditions. The mitochondrial stress test was done as described previously. ${ }^{25}$ Oxygen consumption rate was measured with a XF-96 Flux. Analysis according to manufacturer's instruction. Oxidative phosphorylation characteristics (basal respiration, maximal respiration, ATP production, non-mitochondrial oxygen consumption) were calculated from the oxygen consumption rate differences in response to oligomycin $(1 \mu \mathrm{M}), \operatorname{FCCP}(2,5 \mu \mathrm{M})$, and antimycin $\mathrm{A}+$ Rotenone (1 $\mathrm{\mu M}$ each). After measurements, protein concentration of each well was measured using BCA kit (Pierce, Cat. No. 23227) to correct for different cell density and protein content.

\section{Lactate and pH measurement}

Lactate and $\mathrm{pH}$ were determined in supernatant directly derived from BMDMs cultured under normoxic or hypoxic conditions $\left(1 \% \mathrm{O}_{2}\right)$ for 72 hours in standard RPMI culture medium. Measurements were performed on the GEM 4000 (Werfen, Barcelona, Spain).

\section{ELISA}

Detection of SCAIX in plasma samples was done by commercially available Human Carbonic Anhydrase IX DuoSet ELISA (R\&D Systems, MN, USA) suited to detect $31-2000 \mathrm{pg} / \mathrm{ml}$ CAIX. Briefly, detection of SCAIX in plasma was performed by sandwich ELISA using capture 


\section{3 | CAIX in atherosclerosis}

monoclonal antibody (200ng/ml; $100 \mu \mathrm{l}$ per well) coated on 96-plate and incubated overnight at room temperature. After washing and blocking (1\% BSA in PBS) $100 \mu$ l of plasma sample (diluted 1:1 in reagent diluent) or standards were added and incubated for $2 \mathrm{~h}$ at RT. After washing, $100 \mu$ l of detection antibody $(200 \mathrm{ng} / \mathrm{ml})$ were added and incubated for $2 \mathrm{~h}$ at RT; streptavidin-HRP (100 $\mu$ l) was used as a detector. Reaction was stopped by adding $50 \mu \mathrm{l}$ of the stop solution and the optical density of each well was determined using a microplate reader set to $492 \mathrm{~nm}^{26}$

\section{Genotyping}

CAIX WT and KO stomach samples were used for DNA isolation and genomic DNA was amplified using REDTaq (Sigma, D8312). Primers used for CAIX PCR were the following: CAIX WT1: 5'-CCA GTC AGC TGC ATG GCC-3', WT2: 5'- AGG AGC CTC GGG AGT CGA-3', KO: 5'AGG AGC AAA GCT GCT ATT GG-3'. After PCR, samples were run on a temperature gradient gel $\left(56-65^{\circ} \mathrm{C}\right)$ for further analysis. CAIX WT product is visualized at $\sim 318 \mathrm{bp}$, while CAIX KO product is visualized $\sim 400 \mathrm{bp}$.

\section{Real time quantitative PCR}

Cells were cultured accordingly, and RNA was isolated and produced as described. ${ }^{27} \mathrm{qPCR}$ analyses were performed from $10 \mathrm{ng}$ CDNA using SYBR green (Biorad) and gene specific primers can be found in Supplemental (S) Table 1. Two housekeeping genes (18S and cyclophilin) were used to correct for different mRNA quantities between samples.

\section{Human tissue collection}

Multiple human plaque or plasma collections were used: Maastricht Pathology Tissue Collection (MPTC) including the Maastricht human plaque study (MAASHPS), ${ }^{28}$ and Maastricht human plasma cohort, ${ }^{29}$ the Athero-Express biobank study (www.atheroexpress.nl) ${ }^{30}$, and plasma samples of Cohort On Diabetes and Atherosclerosis Maastricht (CODAM), ${ }^{31-33}$ for analysis of plaque protein and mRNA levels using immunohistochemistry, microarrays, and western blot analysis and plasma CAIX levels.

All patient material collection, storage, and use of tissue and patient data were performed in agreement with the Dutch Code for Proper Secondary Use of Human Tissue (http://www.fmwv.nl). The studies all comply with the Declaration of Helsinki, and the local Medical Ethics Committee in accordance with national regulations approved use of this tissue. To be more precise, MPTC, Maastricht human plaque study, Maastricht human plasma cohort and CODAM were approved by the Medical Ethics Committee of the Maastricht University Medical Centre. The Athero-Express study was approved by Medical Ethics Committee of University Medical Center Utrecht. All included patients gave their written informed consent. 
Maastricht human plaque study (MAASHPS) cohort: Human atherosclerotic plaque samples or serum were obtained from carotid artery lesions from patients undergoing endarterectomy (Department of Vascular Surgery, Maastricht University Medical Center and Zuyderland Medical Center, Sittard-Geleen, the Netherlands). Seven patients were injected with pimonidazole prior to surgery to detect tissue hypoxia. ${ }^{1}$ The MaasHPS (Maastricht Human Plaque Study) consists of 22 patients, who underwent carotid endarterectomy, and comprised the intra-patient, paired comparison of stable segments with thick fibrous cap atheroma's to unstable segments containing intraplaque hemorrhage in the same plaques. Plaques were sequentially divided in alternating samples used for formalin fixation, or snapfrozen for protein or transcriptomic analysis by microarray. Microarray analysis was used to study whole plaque mRNA expression as described elsewhere. ${ }^{34}$ Classification of plaque stage was done according to Virmani et al. ${ }^{35}$ by two investigators independently. After RNA quality check, and re-classification after quantitative morphometry, 16 stable, and 27 unstable segments were included in the analysis. Pearson correlations were calculated between CAIX mRNA expression with several plaque traits in unstable plaques, as determined by plaque histology and immunohistochemistry. For all immunohistochemical purposes, two slides per patient for each stable and unstable segment were used, hence four slides in total. Adjacent tissue sections were phenotyped extensively for plaque size, necrosis, inflammation (CD68, CD3, arginase, iNOS), SMCs and fibroblasts ( $\alpha \mathrm{SMA})$, collagen (Sirius red), macro- and micro-calcification (Alizarin red), and angiogenesis (CD31+ microvessel density, newly formed $\mathrm{CD} 105+\mathrm{Cd} 31+$ double- positive

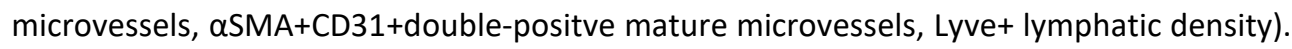
Analysis of each individual staining was performed using Leica Qwin software, and values averaged per segment, resulting in two values per patient, one for the stable and one for the unstable segment.

Maastricht human plasma cohort: A description of the subjects and eligibility criteria of enrolled patients in the original study were described elsewhere. ${ }^{29}$ In short, we selected plasma of a total of 63 patients with either stable or unstable carotid artery plaques, as determined by histology. ${ }^{35}$

Athero-Express Biobank Study: In the Athero-Express Biobank Study, carotid plaques were collected from symptomatic patients for histology and protein lysis. ${ }^{30}$ Tris lysates of 64 human plaques were used for CAIX western blot analysis of which $48(75 \%)$ men, $69 \pm 8$ years old, 27 (42\%) subjects with a secondary event, 42 (66\%) plaques with IPH, 26 (40.6\%) atheroma's, 33 (51.6\%) fibrous plaques and 5 (7.8\%) fibroatheromas. Patient and plaque characteristics in subjects with and without plaque-resident CAIX are described in Supplemental table S2.

CODAM: The CODAM cohort was designed to study associations between diabetes, atherosclerosis and other cardiovascular diseases. An extensive description of the cohort 


\section{3 | CAIX in atherosclerosis}

and execution and description of used measurements can be found elsewhere. ${ }^{31-33}$ In short, 574 subjects with, or at risk for, diabetes mellitus type 2 were enrolled in this study, and followed for 7 years. Any cardiovascular outcomes were documented, and blood was withdrawn at enrollment and after 7 years follow-up. In CODAM, CVD was defined as the occurrence of at least one of the following: previous myocardial infarction (MI); coronary bypass; percutaneous coronary intervention (PCI); stroke or transient ischemic attack reported by questionnaires; signs on a 12-lead electrocardiogram of $\mathrm{MI}$ or ischemia, traumatic limb amputation; and/or an ankle brachial index <0.9. Cardiovascular events (CVE), comprised of $\mathrm{MI}$, stroke, coronary bypass and/or $\mathrm{PCl}$, as reported. ${ }^{36}$ Patient demographics are described in Supplemental table S3.

\section{Human carotid protein isolation and Western Blot}

Human carotid plaques were snap-frozen immediately after collection. Sample preparation procedures were carried out on dry ice. Carotid plaques were divided in smaller pieces $( \pm 0.5 \mathrm{~cm})$ and manually grinded under constant addition of liquid nitrogen. The resulting tissue dust was incubated with $500 \mu$ TRIS lysis buffer and EDTA-free protease inhibitor cocktail (Roche, 04693159001). Subsequently, protein liberation was further enhanced by crushing grinded tissue in a Beadbeater, and sonicating the sample for 2 minutes. Upon centrifugation (maximal speed, 5 minutes) supernatant was collected and protein concentration was determined using BCA kit (Pierce, Cat. No. 23227). Pre-cast gels (ExpressPlus PAGE gel 8-16\%, genscript, M81612) were used for protein seperation, and transferred to a nitrocellulose membrane. CAIX was detected using primary antibody M75 (1:3000) followed by HRP-labeled secondary antibody incubation (Jackson, 715-035-150). Signal was developed using SuperSignal West Femto Maximum Sensitivity Substrate (Thermo Fisher scientific, 34095) and visualized using a digital scanner. Signal intensity was quantified using ImageJ Gel Analyzer software and normalized for total protein content using intensity of Ponceau S. Each gel contained the same plaque sample, allowing normalization between gels.

\section{Immunohistochemical staining}

Human carotid sections $(4 \mu \mathrm{m})$ were deparaffinized, stained with CD68 mouse - anti-human (Dako, GA60961-2) or iNOS rabbit - anti-human (Abcam, ab3523) antibody and stained with vector red alkaline phosphatase kit (Vector, SK-5100). Subsequently, antigen retrieval was performed using citrate buffer pH6.0 (Dako REAL target retrieval, Dako). CAIX was stained with M75 primary antibody and visualized using vector blue alkaline phosphatase kit (Vector, SK-5300). Hypoxia was detected, in patients who received a pimonidazole injection prior to endarterectomy, using HRP labeled rabbit - anti-pimonidazole antibody (PAb2627, NPI). For iNOS detection, rabbit-anti human iNOS antibody (ab3523, Abcam) was used. Hypoxia and iNOS staining were visualized using AEC+ Substrate-Chromogen (K3461, 
Agilent). Prior to Entallan mount, slides were dehydrated using Imsol (diluted 1:5) on the hot plate $\left(37^{\circ} \mathrm{C}\right)$.

\section{Statistical analysis}

sCAIX plasma values were, even after log transformation, not normally distributed; moreover SCAIX was only detectable in 80 of 572 plasma samples. Therefore, we performed subsequent regression analyses with SCAIX as a dichotomous independent variable (sCAIX detectable yes/no). Cross-sectional analysis was performed to assess prevalent CVD and CVE, as defined in CODAM. Logistic regression (IBM SPSS Statistics, Version 25.0.) was used to test association between SCAIX and presence of CVD or CVE. To this end, the following models were employed. Model 1: Crude, no adjustments. Model 2: model $1+$ adjustments for sex and age. Model 3: model $2+$ adjustments for smoking (status [current, former, never] \& packyears), medication (lipid-modifying $\mathrm{y} / \mathrm{n}$, anti-HT $\mathrm{y} / \mathrm{n}$, glucose-lowering $\mathrm{y} / \mathrm{n}$ ), glucose metabolism status (IGM y/n, DM2 y/n). Linear regression was performed to study the association between sCAIX (detectable yes/no) and Intima-media thickness of the carotid artery (CIMT) and ankle-arm index (AAIX). To assess prognostic value of SCAIX, we performed a prospective analysis, with outcome measures CVD and CVE as determined on the end of follow-up period. Here, similar logistic regression models were employed. Only subjects that did not have CVD $(n=317)$ or CVE $(n=369)$ at time of inclusion were included in this analysis.

Distribution of plaque-resident CAIX was also not normal, and positively skewed. Square root transformation rescued skewness, but CAIX distribution remained not normal as shown by Shapiro-Wilk normality test. Non-parametric Mann-Whitney rank sum tests were done to test if CAIX was statistically different between plaques with a single or secondary event, with and without IPH, and between atheroma's and fibrous plaques. In addition, dichotomous analysis of plaque CAIX using Fisher's exact test was performed.

All in vitro data are presented as mean+SEM, with *p-value $<0.05, * * p$-value $<0.01, * * * p$ value $<0.0001$. All parameters were analyzed using independent sample tests and were tested for normal distribution using Shapiro-Wilk normality test. Parameters with two groups were compared with student's t-test or Mann-Whitney rank-sum test. 


\section{3 | CAIX in atherosclerosis}

\section{Results}

CAIX is present in human atherosclerotic plaques and correlated with pro-atherogenic traits

As the expression pattern of CAIX in atherosclerosis was unknown, and macrophages are the predominant hypoxic cells expected to express CAIX, we performed double immunohistochemical staining for CAIX and CD68 to evaluate their co-localization in human unstable carotid plaques (Figure 1A-C). CAIX immunoreactivity was detected on only $5-10 \%$ of the plaque surface area (Figure 1B, Supplemental Figure S2). If present, CAIX was observed in the macrophage-rich shoulder regions of the plaque, as well as in the thick fibrous cap. CD68-negative, spindle-like cells reflecting smooth muscle cells and/or fibroblasts were also positive for CAIX. As expected, CAIX expression was positive in pimonidazole-positive, hypoxic regions in the human plaque (Figure 1D). Furthermore, CAIX mRNA expression in unstable human plaques correlated with pro-inflammatory macrophages (iNOS/CD68), lipid core size, and CD105+ new angiogenic microvasculature (Figure 1F). The former was validated by a double staining for iNOS and CAIX, showing coexpression by foamy cells in the hypoxic plaque region as indicated by the arrows (Figure 1E). The correlation with new angiogenic sprouts, likely also a response to hypoxia, might reflect the hypoxia-responsive nature of CAIX. These data suggested an association of CAIX with plaque hypoxia and inflammation, and warranted further investigation of a role in macrophage function, and potential as biomarker. 
A
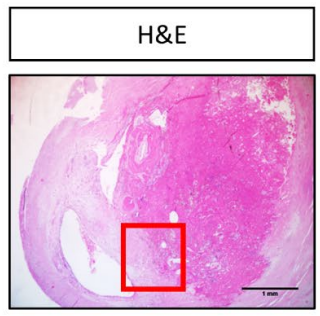

D
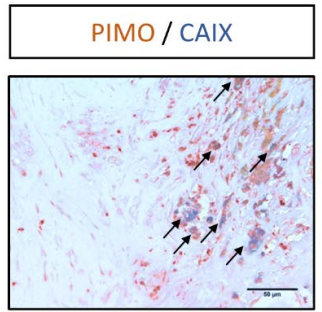

B

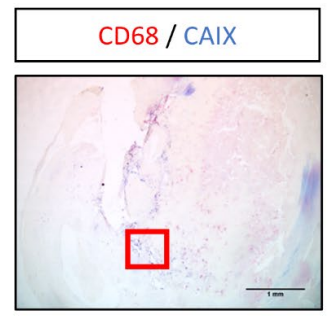

E

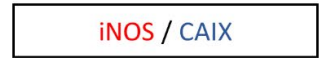

$\mathbf{F}$

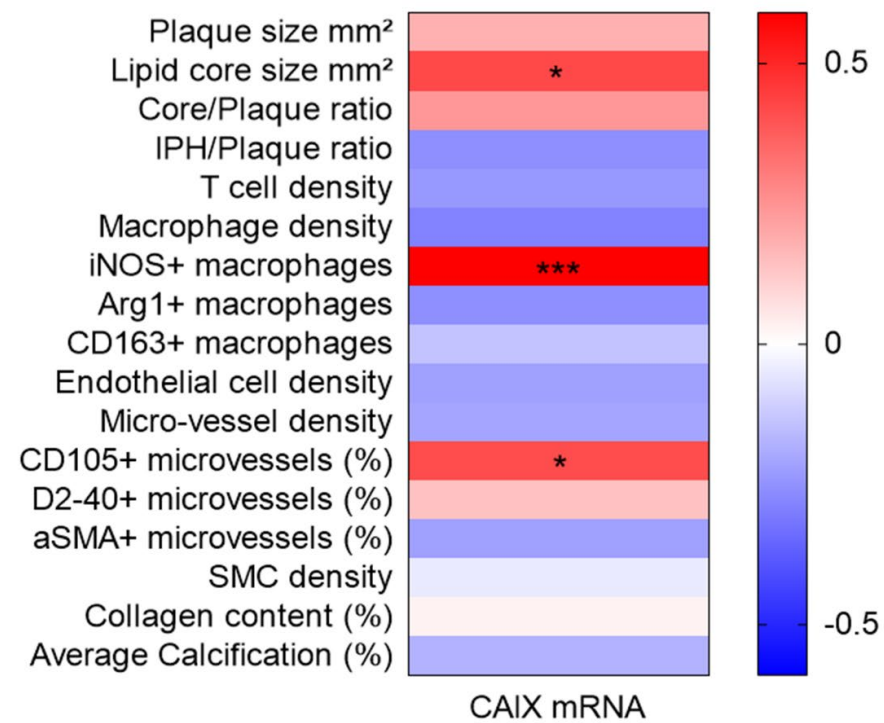

Figure 1. CAIX was present in human atherosclerotic plaque and co-localized with $\mathrm{CD}^{+} 8^{+}$cells (A) Microphotograph of hematoxylin and eosin (H\&E) stained human unstable carotid artery plaque. Red square represents region that was magnified in all stainings. (B) Double staining of CAIX (blue) and CD68 (red) of unstable human atherosclerotic plaque, magnification of red square is depicted in (C). Arrows indicate double positive cells. (D) Unstable human atherosclerotic plaque of patient injected with Pimonadizole to detect hypoxia (brown) or (E) iNOS (red), and CAIX (blue). Arrows indicate double-positive areas accompanied by an additional zoomed in area. (F) Heatmap of Pearson correlations of CAIX mRNA in unstable human plaque segments $(n=23)$ with plaque traits, determined on adjacent histology slides. All plaque sections originate from the MaasHPS cohort. Arg1; arginase 1. aSMA; alpha smooth muscle cell actin. D2-40; podoplanin. iNOS; inducible nitric oxide synthase. IPH; intra-plaque hemorrhage. MVD, microvessel density NA; not applicable. ${ }^{*} p<0.05,{ }^{* *} p<0.01$, $* * * p<0.001$. 


\section{3 | CAIX in atherosclerosis}

\section{CAIX deficiency alters macrophage metabolism}

From the extensive literature available from the cancer field, we know that CAIX is involved in $\mathrm{pH}$ regulation, thereby enabling metabolic changes in cancer cells. As we observed a correlation with iNOS+ macrophages, we reasoned that CAIX deficiency might also interfere with macrophage metabolism, in this way affecting atherosclerosis relevant functions. Therefore we isolated BMDM from CAIXKO mice. ${ }^{22}$ First, we confirmed gene knock-out in stomach tissue from CAIXko mice compared to WT mice (Supplemental (S) Figure S1).

Thereafter, we assessed if CAIX harbors a function in $\mathrm{pH}$ regulation also in BMDM. As expected, the $\mathrm{pH}$ of CAIXKO BMDM cell culture supernatant was enhanced, and lactate content was lowered, compared to control BMDMs, confirming the functional knockout of CAIX in BMDM (Figure 2A-B). Energy metabolism was assessed using the seahorse XF analyzer. CAIXKO BMDMS were not able to produce similar oxygen consumption rates as WT BMDM upon mitochondrial oxidative phosphorylation uncoupling. This so-called respiratory spare capacity was significantly lowered in CAIXKO BMDMs, while baseline ATP production was not altered (Figure 2C-D). Nevertheless, the reduction of respiratory capacity did not affect cell proliferation measured by impedance and EdU incorporation (Figure 2E-F). Macrophages undergo several metabolic changes in order to polarize successfully towards pro- or anti-inflammatory phenotypes ${ }^{37,} 38$ and CAIX mRNA expression also correlated with pro-inflammatory macrophage presence in human plaque (Figure 1F). We thus assessed markers of pro- and anti-inflammatory macrophages on mRNA level in BMDMs treated with LPS + IFN $\gamma$. No significant change in pro- and anti-inflammatory gene expression has been observed between CAIX and WT cells (Figure 2G-L), nor did M1 or M2 cytokines change CAIX expression (Figure $\mathbf{2 M}$ ). Together, these data support a role for CAIX in $\mathrm{pH}$ regulation and a lower ability of their oxidative phosphorylation machinery to respond to a sudden increase in energy demand. 


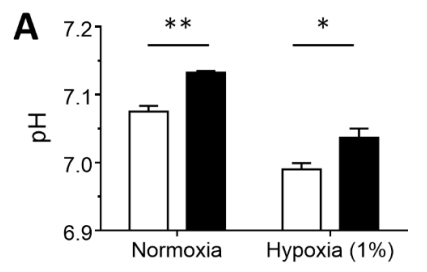

C

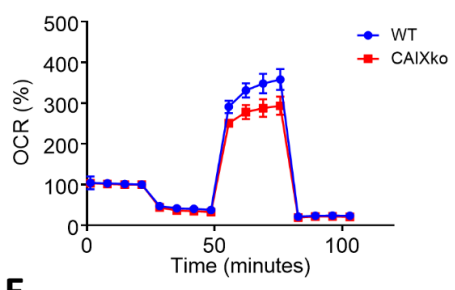

$\mathbf{E}$

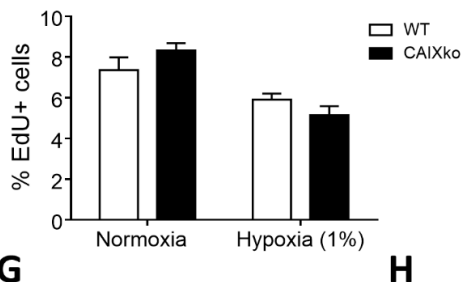

G

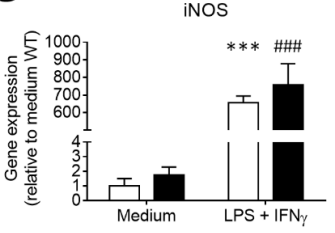

J

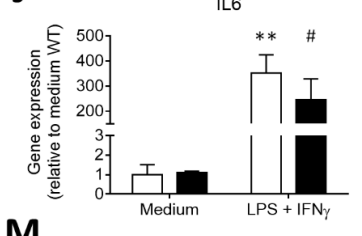

M

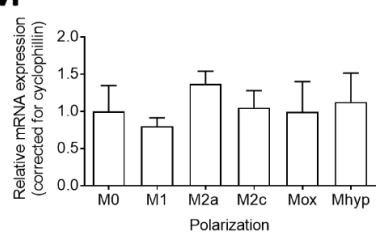

H

$\mathbf{K}$

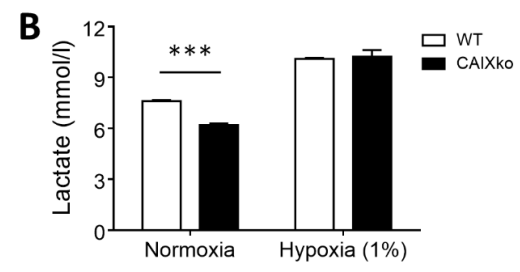

D

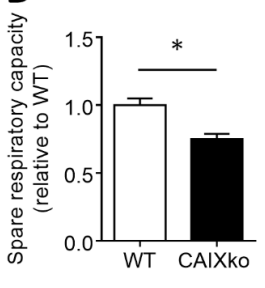

$\mathbf{F}$
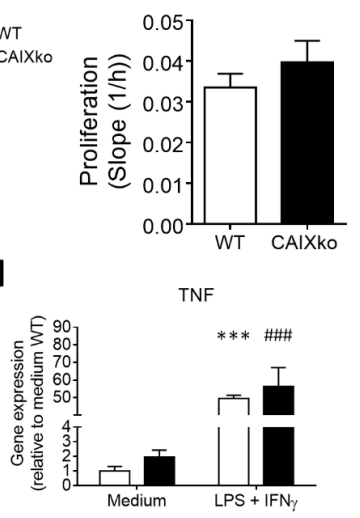

I
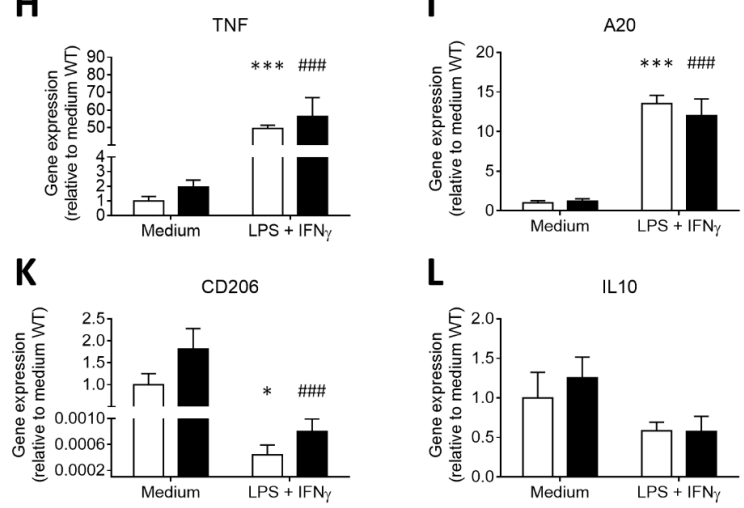

$\mathbf{L}$

口 ${ }_{\text {CAIXko }}$

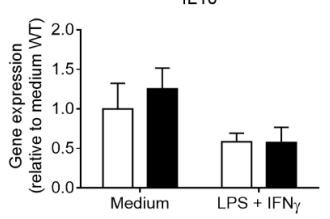

Figure 2. CAIXKO reduced spare respiratory capacity in BMDMs (A) pH and (B) lactate measurement of BMDM culture medium $(n=4)$ after 72 hours of incubation in either normoxia or hypoxia $\left(1 \% \mathrm{O}_{2}\right)$. (C) Representative graph of Seahorse mitochondrial stress assay of BMDMs ( $n=5 /$ group, 1 experiments). Analysis was performed on data corrected for protein content in each well and with baseline measurement 4. (D) BMDM spare respiratory capacity displayed relatively to WT. (E) BMDM 


\section{3 | CAIX in atherosclerosis}

proliferation measured by EdU incorporation $(n=4)$ after 24 hours incubation in normoxia or hypoxia $\left(1 \% \mathrm{O}_{2}\right)$. (F) Mean BMDM proliferation measured by real-time impedance $(n=5)$ over 72 hours. Slope represents the increment of impedance over time. (G) Real time quantitative PCR of pro-inflammatory genes inducible nitric oxide synthase (iNOS), (H) tumor necrosis factor (TNF), (I) Tumor necrosis factorinduced protein 3 (A20) (J) interleukin 6 (IL6) and anti-inflammatory genes (K) mannose receptor (CD206) and (L) interleukin 10 (IL10) in non-stimulated (medium) and LPS + IFNץ stimulated BMDMs. All values are relative to WT unstimulated BMDMs of respective target gene. (M) CAIX mRNA expression in polarized BMDMs. * Indicates a significant difference of the given condition compared to WT medium treated BMDMs. \# Indicates significant difference of the given condition compared to CAIXKO medium treated BMDMs. No difference was observed between WT and CIX-ko BMDM (white and black bars respectively). All results show mean \pm SEM. ${ }^{*} p<0.05,{ }^{* *} p<0.01,{ }^{* * *} p<0.001$.

\section{CAIXKO macrophages do not show a distinct pro-atherogenic phenotype}

Since CAIX mRNA correlated with pro-atherogenic plaque traits, and CAIXKO led to metabolic changes in BMDMs, we further investigated its role in macrophage functions relevant for atherosclerosis. As expression of CAIX mRNA in human unstable plaque segments correlated with lipid-rich necrotic core size, we explored BMDM apoptosis and lipid uptake. BMDM apoptosis in response to 7-ketocholesterol, was unaffected in both normoxia and hypoxia (Figure $\mathbf{3 A}-\mathbf{C}$ ). As confirmation, there was no clear correlation between CAIX mRNA and genes involved in apoptosis, in human unstable plaque segments. Only 10 of 158 genes included in the "hallmarks of apoptosis" gene set derived from gene set enrichment analysis were significantly correlated with CAIX mRNA (Supplemental Table S2).In addition, lipid uptake by CAIXKO BMDMs was not significantly altered both in normoxia and hypoxia (Figure 3D-F). Together, CAIXko in macrophages did not change the atherosclerosis-relevant functions studied here, despite having small changes in metabolism. 
A
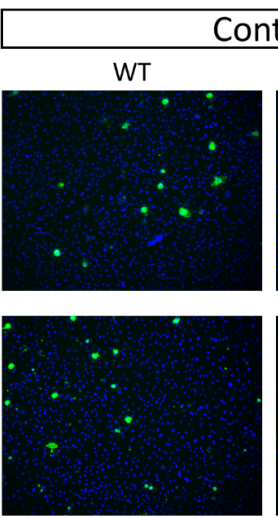

Normoxia

B

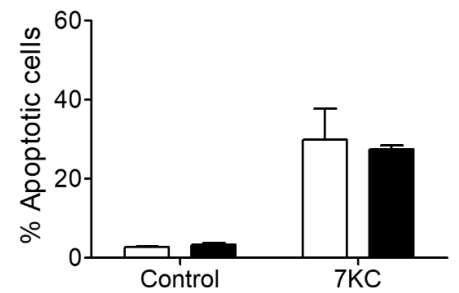

D
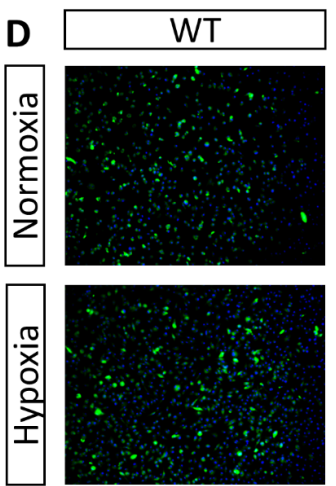
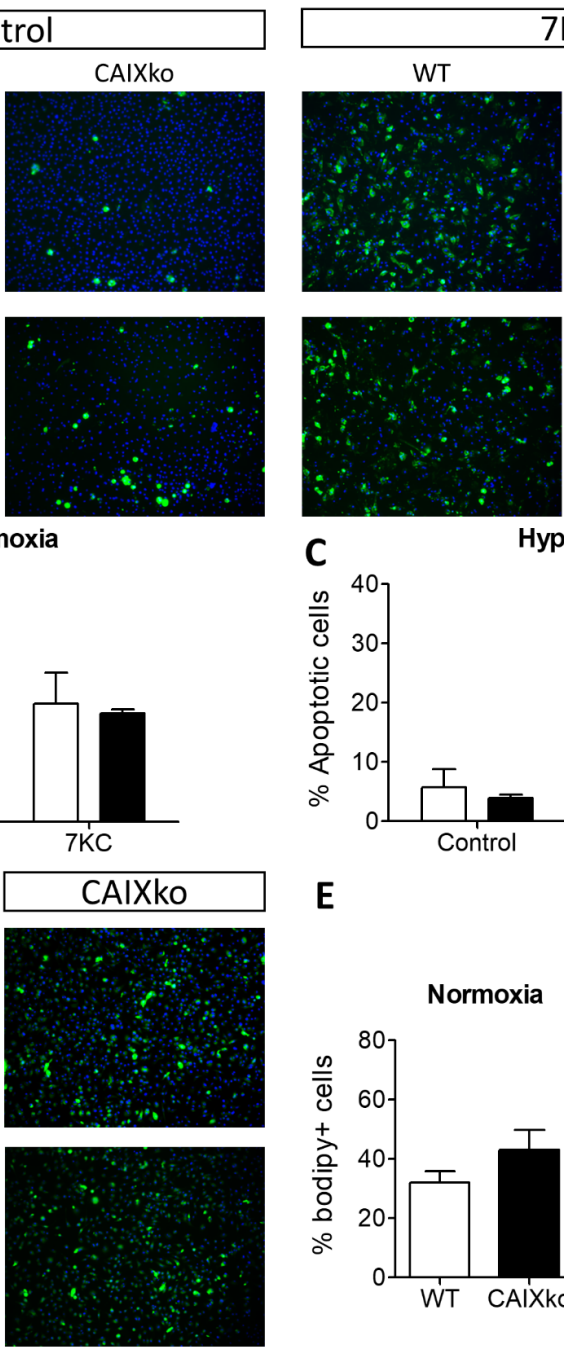

$7 \mathrm{KC}$
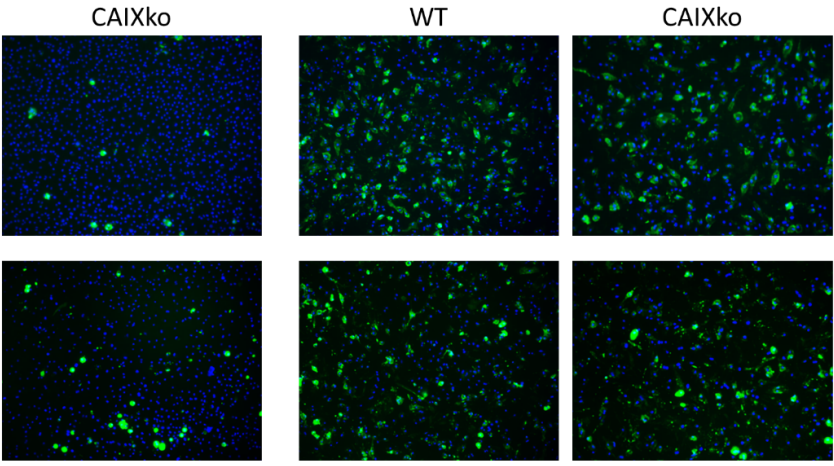

C

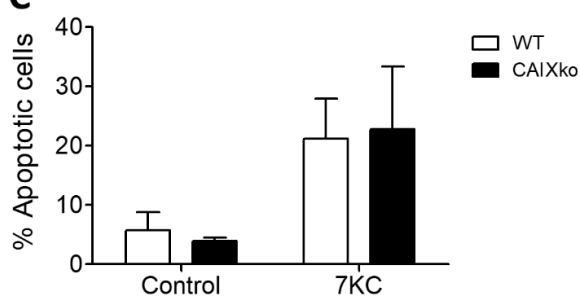

$E$

$\mathbf{F}$
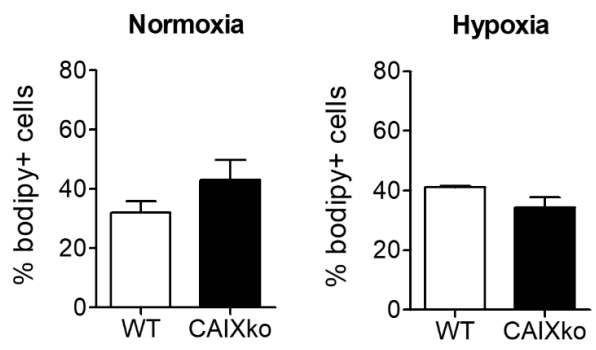

Figure 3. CAIXKO did not alter BMDM apoptosis or lipid uptake. Apoptosis was induced by incubating cells with $50 \mu \mathrm{M}$ 7-ketocholesterol for 24 hours in all experiments. (A) Representative photographs of apoptotic cells (annexin positive) in WT and CAIXKO BMDMs in normoxia and hypoxia $\left(1 \% \mathrm{O}_{2}\right)$. (B) $\operatorname{BMDM}(n=3)$ apoptosis in normoxia or (C) hypoxia ( $1 \% \mathrm{O}_{2}, 24$ hours). (D) Representative photographs of BMDMs after lipid uptake (Topfluor positive) in WT and CAIXKO BMDMs in normoxia and hypoxia $\left(1 \% \mathrm{O}_{2}\right)$. (E) BMDM lipid uptake in normoxia $(n=3)$ or $(\mathbf{F})$ hypoxia. All results show mean $\pm \mathrm{SEM}$.

\section{Baseline SCAIX did not associate with future primary cardiovascular disease outcome measures}

Although a role for CAIX in macrophage function seemed limited, its expression patterns and hypoxia-selective shedding, suggested to explore whether the soluble CAIX (SCAIX) or plaque-resident CAIX were associated with cardiovascular outcome measures. We studied 


\section{3 | CAIX in atherosclerosis}

the association between plasma SCAIX and CVD in 579 asymptomatic patients with future, primary events in the Cohort on Diabetes and Atherosclerosis Maastricht (CODAM). First, plasma of CODAM was collected at inclusion of asymptomatic patients, and plasma CAIX measured at baseline was associated with future, primary events during 7 years follow-up. Plasma sCAIX was detectable in only $14 \%$ of participants from the CODAM cohort, and there was no significant association between the detectability of SCAIX, and cardiovascular outcome measures (Table S4). As can be appreciated from Table 1 and Table S4, CAIX was unable to predict prevalence or incidence of CVD or CVE in the CODAM, regardless of the model.

Table 1. Association of SCAIX with prevalent CVD and CVE

\begin{tabular}{|l|l|l|l|}
\hline CVD & OR & \multicolumn{1}{l}{ 95\%; Cl } & p-value \\
\hline Model 1 & 0.80 & $0.461 ; 1.401$ & 0.441 \\
\hline Model 2 & 0.72 & $0.405 ; 1.277$ & 0.261 \\
\hline Model 3 & 0.97 & $0.510 ; 1.837$ & 0.921 \\
\hline CVE & OR & 95\%; Cl & p-value \\
\hline Model 1 & 0.75 & $0.370 ; 2.525$ & 0.428 \\
\hline Model 2 & 0.66 & $0.317 ; 1.281$ & 0.271 \\
\hline Model 3 & 1.07 & $0.430 ; 2.671$ & 0.881 \\
\hline
\end{tabular}

Logistic regression analysis using 572 subjects for model 1 and 2, 560 subjects for model 3 , s packyears had 12 missing values. sCAIX was treated as dichotomous independent variable (detectable yes/no, yes, $n=80$ ). Model 1: Crude, no adjustments. Model 2: model $1+$ adjustment for sex and age. Model 3: model 2 + adjustments for smoking (status [current, former, never] \& packyears), medication (lipidmodifying $y / n$, anti-HT $y / n$, glucose-lowering $y / n$ ), glucose metabolism status (IGM y/n, DM2 y/n).

We then investigated a potential relationship between CAIX and plaque burden, as it is well conceivable that enhanced total body plaque burden would lead to enhanced circulating levels of SCAIX. We thus used intima-media thickness of the carotid artery (cIMT) and anklearm index (AAIx) as measures for carotid and peripheral artery plaque burden, respectively. However, linear regression analysis did not reveal an association between SCAIX and plaque burden (Table 2).

Table 2. Association of sCAIX with cIMT and AAIX

\begin{tabular}{|l|ll|l|}
\hline clMT & $\boldsymbol{\beta}$ & $\mathbf{9 5 \%} ; \mathbf{C l}$ & $\boldsymbol{p}$-value \\
\hline Model 1 & 0.027 & $-0.011 ; 0.066$ & 0.166 \\
\hline Model 2 & 0.021 & $-0.016 ; 0.058$ & 0.275 \\
\hline Model 3 & 0.026 & $-0.012 ; 0.064$ & 0.185 \\
\hline AAlx & $\boldsymbol{\beta}$ & $\mathbf{9 5 \%} \mathbf{C l}$ & $\boldsymbol{p}$-value \\
\hline Model 1 & -0.006 & $-0.037 ; 0.024$ & 0.689 \\
\hline Model 2 & -0.001 & $-0.031 ; 0.028$ & 0.938 \\
\hline Model 3 & -0.009 & $-0.038 ; 0.020$ & 0.550 \\
\hline
\end{tabular}


Linear regression analysis using 504 and 541 subjects that underwent cIMT and AAlx measurement, respectively. SCAIX was treated as dichotomous independent variable (detectable yes/no). $\beta$ indicates mutation of dependent variable if CAIX is detectable (yes). Model 1: Crude, no adjustments. Model 2: model $1+$ adjustment for sex and age. Model 3: model 2 + adjustments for smoking (status [current, former, never] \& packyears), medication (lipid-modifying $y / n$, anti-HT y/n, glucose-lowering $y / n$ ), glucose metabolism status (IGM y/n, DM2 y/n).

As hypoxic tumors are a known source of SCAIX, ${ }^{26,39,40}$ we assessed cancer prevalence. The presence of cancer could not have influenced our data, since 17 (3.5\%) subjects with undetectable sCAIX reported active cancer, compared to 3 subjects with detectable levels (3.8\%). Together, sCAIX levels were frequently undetectable excluding it as a meaningful biomarker of cardiovascular disease outcome measures in the CODAM cohort, and this was not biased by the presence of hypoxic tumors.

\section{sCAIX levels were similar in symptomatic patients with symptomatic plaques with and without intraplaque hemorrhage}

As SCAIX did not predict future, primary CVD or correlated with atherosclerotic burden in patients with asymptomatic disease at the time of plasma collection in the CODAM cohort, we measured SCAIX in plasma samples of recently symptomatic patients with $(\mathrm{N}=35)$ or without $(\mathrm{N}=28)$ histological evidence of intraplaque hemorrhage (IPH) in their carotid plaques, collected during carotid endarterectomy (MPTC). ${ }^{29}$ Participants from this cohort were known to have severe, symptomatic atherosclerosis, with expected increase in plaque hypoxia, and hence might be a more suitable population to detect sufficient levels of SCAIX. Therefore, we investigated if sCAIX could distinguish between stages of plaque severity. The detectable fraction of SCAIX was indeed higher in these recently symptomatic patients compared to the participants in CODAM. Unfortunately, SCAIX levels remained largely undetectable in plaques with or without IPH from these symptomatic patients $(45.7 \%$ and $46.4 \%$ respectively), and median levels were also similar (Figure $4 \mathrm{~A}$ ). In conclusion, there is no difference in SCAIX levels between symptomatic patients presenting with or without IPH in carotid plaques.

\section{Plaque-resident CAIX protein was similar in participants with and without secondary events}

sCAIX could neither predict cardiovascular outcome measures, nor distinguish between patients with stable versus unstable plaques. Interestingly, sCAIX failed to predict disease progression in multiple types of cancer, whereas tumor bound CAIX did. ${ }^{39}, 40$ Therefore we tested the hypothesis that plaque-resident CAIX does have prognostic value for future CVD, as has been shown for other plaque constituents e.g. osteopontin ${ }^{41}$, and fatty acid-binding protein $4^{42}$ in the Athero-Express cohort. This cohort consists of plaques collected from symptomatic patients at carotid endarterectomy for plaque phenotyping and protein 


\section{3 | CAIX in atherosclerosis}

expression analysis. Participants were followed up to register secondary cardiovascular events, and plaque phenotype and expression of certain proteins were found to predict these secondary events. ${ }^{30,41,42}$ In line with the detectable fraction of sCAIX in symptomatic patients in the Maastricht MPTC plasma cohort, plaque resident CAIX was only detected in $53.1 \%$ of plaques. This is in agreement with the non-abundant expression of $5-10 \%$ positive surface area of CAIX immunoreactivity in the MaasHPS cohort (Figure S2). Moreover, plaque resident CAIX was similar between cases with just a primary event versus cases with a secondary clinical event from any vascular bed, and between unstable plaques with intraplaque hemorrhage (IPH) and stable plaques, or with high or low inflammatory burden (Figure 4B-C, Table S5 and Figure S2). CAIX protein levels were also similar in lipid-rich, atheromatous plaques with a high inflammatory content compared to lipid-poor, fibrous plaques with high collagen content (Figure 4D-E). Moreover, CAIX mRNA was also similar between human carotid plaques with and without IPH, albeit in a different patient group. Together, expression of plaque resident CAIX was not abundant and if detectable, did not associate with future CVD and did not distinguish between plaque types, in line with sCAIX. 
A

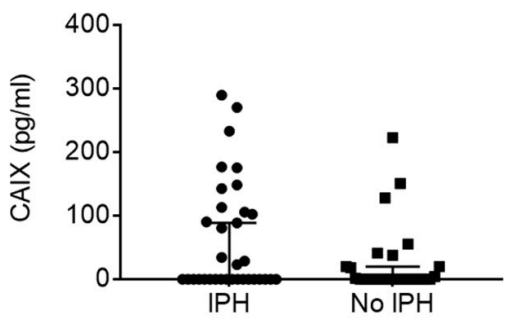

B

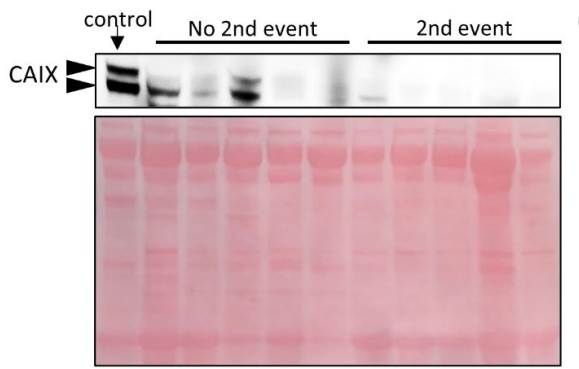

C

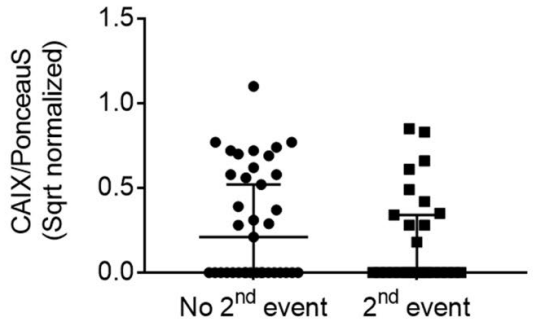

D

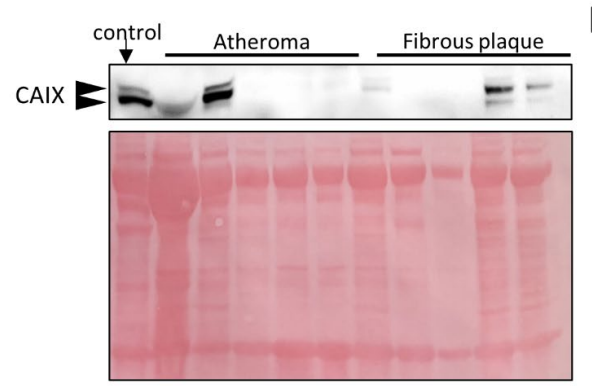

E
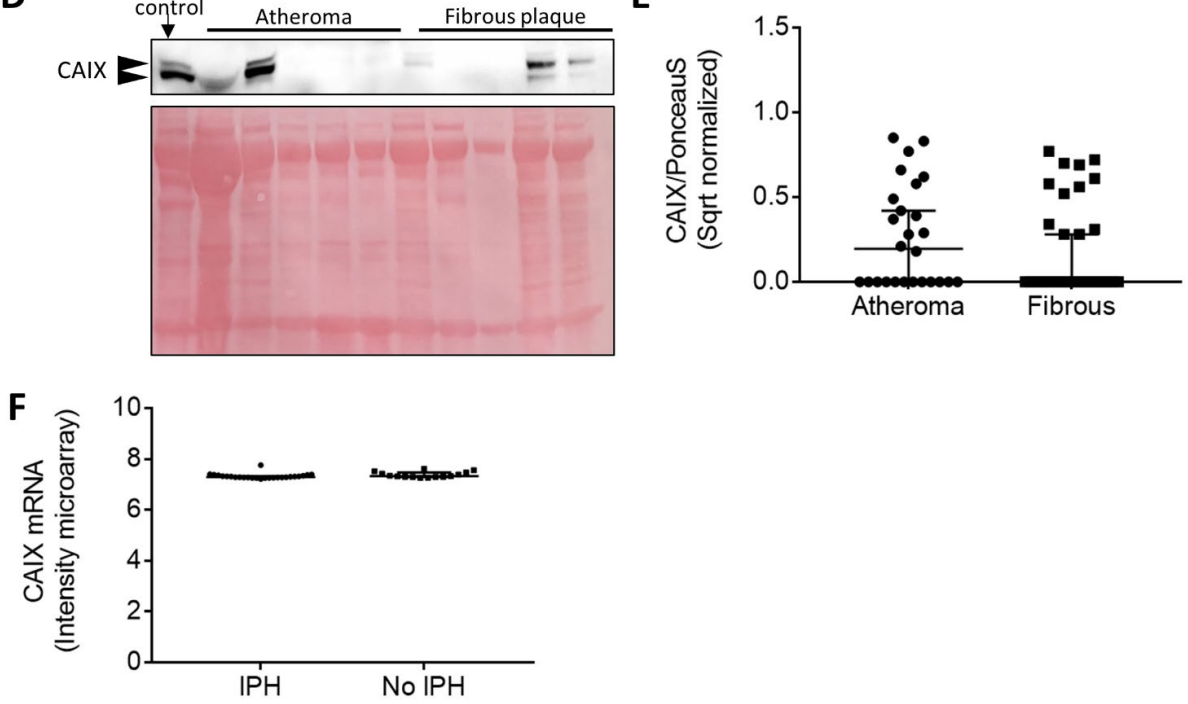

Figure 4. SCAIX and plaque resident CAIX was not changed in patients with events or plaque type (A) SCAIX values in human carotid plaques with or without IPH from the MaasHPS cohort. (B) Representative cropped western blot and (C) quantification of CAIX protein in human carotid plaque lysates with ( $n=27)$ and without a second event $(n=35)$ derived from Athero-Express biobank study. CAIX protein intensity is corrected for protein levels by ponceau $S$, and square root (sqrt) normalized. (D) Representative cropped western blot and (E) quantification of CAIX protein in atheromatous (26) and fibrous $(n=33)$ human carotid plaque lysates derived from Athero-Express biobank study. Arrow heads indicate two bands known to correspond to CAIX, 2nd, second. Full length western blots can be found in Supplemental Figures S3 and S4, (F) CAIX mRNA as derived from microarray analysis in human carotid plaques with or without IPH derived from the MaasHPS biobank. 


\section{3 | CAIX in atherosclerosis}

\section{Discussion}

In this study, we used multiple human cohorts and murine macrophages to study expression, function and biomarker potential of CAIX in CVD. CAIX was expressed in human atherosclerotic plaques with intraplaque hemorrhage and co-localized with CD68+, iNOS+ macrophages and hypoxia. In addition, CAIX deficiency in BMDM led to reduced spare respiratory capacity. However, macrophage polarization, lipid uptake properties, or apoptosis rate in normoxia and hypoxia were not affected by CAIX knockout. Given its colocalization with hypoxia, and plaque hypoxia correlating with total lesion burden and lesion stability, we explored the use of plaque-resident CAIX and circulating SCAIX, as a biomarker for cardiovascular disease outcome. However, both CAIX and SCAIX were undetectable in the majority of cases, excluding them as a reliable biomarker. If detectable, CAIX was similar between plaque types, and between participants who were event-free during follow-up versus those with recurrent CVE.

In our study, CAIX mRNA expression correlated with pro-inflammatory iNOS+ macrophages, but not with CD163+ or Arginase+ M2 macrophages in atherosclerosis. In contrast, in human cervical cancer CAIX correlated with CD163+ tumor-associated macrophages. ${ }^{43} \mathrm{~A}$ possible explanation may be the known lipid-laden, and pro-inflammatory cell and cytokine environment of the plaque, which greatly facilitates the M1 polarization. Moreover, the plaque contains modified lipids, triggering phagocytic reactions to non-self epitopes. In contrast, mutated, yet endogenous, tumor cells, evade immune cell recognition and phagocytosis by production of suppressive cytokines. ${ }^{44} \mathrm{~A}$ direct comparison of plaque macrophages and tumor-associated macrophages, e.g. by single cell RNA sequencing analysis, could shed more light on their comparison, but is yet lacking.

Despite the association of CAIX with iNOS in human plaques, our study surprisingly fails to show any effect of CAIX deficiency on macrophage polarization. Lipid uptake, proliferation and apoptosis both in normoxia and hypoxia were also not affected by CAIX deficiency. This also contrasts to tumor CAIX, which correlates with tumor cell survival. ${ }^{45}$ Unlike tumor cells, macrophage survival under stress seems independent of CAIX function. Of course, this is not an exhaustive analysis of all macrophage functions pertaining to atherogenesis. Even though we did observe small metabolic effects of CAIXKO, this did not interfere with macrophage function in relation to atherogenesis, as evaluated by survival and polarization. It could be the case that discrepancies between in vitro metabolic function and in vivo association data are caused by other cells involved in plaque development, as CAIX expression is not limited to macrophages, and mRNA was derived from total plaque lysates. Cellular communication in the atherosclerotic plaque has been shown to influence cell density and reactivity to stimuli. ${ }^{46}$ Another explanation could be the inconsistency between CAIX mRNA and protein levels, which has already been indicated in numerous other organs, including kidney, colon and muscle. ${ }^{47}$ Although our conclusion on CAIX function is limited to 
in vitro studies, together with our human in vivo associative results, this suggest only a limited function of CAIX in macrophage biology in the setting of atherosclerosis. In fact, the effect of another $\mathrm{pH}$ regulator, $\mathrm{Na}+\mathrm{H}+$ exchanger 1 , was shown in vitro and in vivo, possibly suggesting its dominant role in $\mathrm{pH}$ regulation in plaque macrophages. ${ }^{21}$

We investigated the association of SCAIX and plaque-resident CAIX, in view of its tight association with plaque hypoxia, with cardiovascular disease outcome measures as determined in CODAM and in Athero-Express. We did not find any association between SCAIX and pre-specified outcomes in subjects of the CODAM cohort. In the majority of cases sCAIX was actually undetectable, which is surprising in light of overt plaque hypoxia expected to upregulate CAIX. ${ }^{1}$ As specificity and detection sensitivity of the CAIX ELISA is high $(>30 \mathrm{pg} / \mathrm{ml})$, it is unlikely that the low detection rate is an artifact due to the technology used. In accordance, expression of plaque-resident CAIX was also limited, as demonstrated by immunohistochemistry and western blotting. There seems to be a discrepancy between mRNA and protein levels, which may be partly explained by the high sensitivity of microarray technology to detect mRNA versus lower sensitive protein detection. In addition, CAIX protein stability and/or shedding may be compromised in atherosclerosis. CAXI shedding is regulated by its sheddase ADAM17, whose activity in turn is negatively affected by extracellular acidity and TIMP3 activity. ${ }^{48,49}$ ADAM17 is expressed in human atherosclerosis and even higher in unstable plaques. ${ }^{50}$ However, its activity may be compromised as plaques indeed show low extracellular $\mathrm{pH}$ and TIMP3 expression, ${ }^{51,52}$ possibly explaining the high frequency of undetectable sCAIX.

The biomarker potential of SCAIX to predict cardiovascular disease or event risk in a nonacute setting seems limited. Importantly, two recent studies uncovered that circulating SCAIX does not correlate with tissue CAIX expression in non-small lung cancer and oral squamous cell cancer tissue. ${ }^{39,40}$ Alternatively, we analyzed CAIX in plaque protein lysates of human carotid arteries. The advantage over SCAIX would be that other sources of SCAIX that can 'pollute' the measurement, are excluded. Nevertheless, plaque-resident CAIX in plaques obtained after a first CVE were also undetectable in half of the cases. Detectable CAIX did not predict recurrence of symptoms over time, nor distinguish between plaque types or plaque traits, like extent of inflammation or IPH in the Athero-Express cohort. ${ }^{53}$ CAIX in coronary plaque lysates was not studied, although parameters in the CODAM cohort did reflect overall CVD across vascular beds. Hence, the association of CAIX with hypoxic plaque macrophages is likely a mere consequence of plaque hypoxia to prevent intracellular acidification. However, as "absence of evidence, is not evidence of absence", the CAIX and sCAIX levels below the detection threshold of our protein assays do not fully exclude a correlation with human disease progression and clinical events. We can merely conclude that CAIX and SCAIX are not suitable as biomarkers for CVD with currently available assays. 


\section{3 | CAIX in atherosclerosis}

One limitation of the current study is the discrepancies between the cohorts in SCAIX detection. sCAIX was detected in roughly $45 \%$ of symptomatic patients (MPTC cohort), while sCAUX was only detected in $14 \%$ of asymptomatic patients (CODAM cohort). Discrepancies between the two cohorts reside in the time of plasma collection an $d$ symptomatic versus asymptomatic patients. It could very well be that plaque progression, and hence aggravated hypoxia, could influence the presence of SCAIX, however, it is important to note that IPH did not alter SCAIX levels. Although the number of included patients is reasonably higher in the CODAM cohort, it is more likely that clinical parameters, e.g. symptomatic vs. asymptomatic, are responsible for the discrepancies between the two cohorts. Another possible limitation of the CODAM cohort for CAIX detection includes the presence of adiposity among its participants. At moment of inclusion, there was no difference in the presence of self-reported cancer between both groups (i.e. sCAIX detectable, nondetectable). Thus, active cancer probably did not play a role in the detection of sCAIX. Adipose tissue is considered to be hypoxic ${ }^{54}$ due to impaired vascularization, with enhanced levels of HIF1 $\alpha$ expression, which could hence be a non-specific source of sCAIX. However, there is also evidence that oxygen tension is not reduced in adipose tissue of obese compared to lean human individuals ${ }^{55}$. Moreover, BMI showed no association with sCAIX in the CODAM study, suggesting limited influence of possible adipose tissue hypoxia on circulating SCAIX.

The study is also limited to populations with elderly participants, at high risk of CVE. In addition, the involvement of CAIX in disease initiation was not studied here, while murine plaque macrophages are already hypoxic in early, fatty streaks.3 Future studies of atherosclerotic plaque initiation in CAIXko mice on a hypercholesterolemic background would yield a definitive answer. Also, investigation of upstream oxygen sensors, like the prolyl hydroxylase enzymes, and their macrophage function, and association with CVD, might provide a better hypoxia biomarker. Alternatively, a combination of hypoxia and/or other markers may be predictive. Together, the conclusions from this study are limited to progression of existing human carotid disease in elderly, high-risk participants. Nevertheless, this is currently most relevant for the clinical practice where established disease is treated. It is also relevant for cancer patients with established CV co-morbidities, being treated with anti-CAIX therapy. 9 The possible lack of association of CAIX with CVD presents a window of CVD safety for this treatment, and relieves concerns of future treatment in the elderly population with multi-morbidities.

In conclusion, CAIXKO did not impact BMDM proliferation, apoptosis or lipid uptake, despite small metabolic effects. In addition, circulating SCAIX or plaque-resident CAIX levels were very low and hence not suitable as biomarkers of cardiovascular outcome. CAIX expression is likely a response of hypoxic plaque macrophages without major consequences for future human CVD in elderly, high-risk subjects. 


\section{Acknowledgements and funding}

The authors would like to thank dr. Oosterwijk (Radboud MC, Nijmegen, The Netherlands) for his advice on human CAIX immunohistochemical staining and western blot. We thank Reetta Vuolteenaho (University of Oulu, Finland) and Maarit Patrikainen (Tampere University, Finland) for genotyping, facilitating tissue isolation and transport of mouse material. In addition, the authors would like to thank the students Melissa Brandts and Thais Abud for their technical assistance, and prof. Andrew Baker for discussions. This work has been supported by the Netherlands Scientific Organization (NWO VIDI 91718364 to J.C. Sluimer), Dutch heart Foundation Dr. Dekker fellowhips (2016T060 to JC Sluimer) and CARIM HS BAFTA for 'talented future PhD candidates' (to JAF Demandt, 2018).

\section{Author contributions}

JAFD and JCS conceived and designed the study. JAFD, LD, KvK, and HJ performed experiments and/or analyzed data. Human CODAM cohort design and acquisition was done by CDAS, CGS, CJHvdK, MMJvG, for Athero-express by GP and SWvdL, for MPTC samples by BMEM, JCS, KBJMC and EALB. SPar supplied CAIX KO bone marrow for macrophage culturing. CPMR kindly provided AnXA5-FP488 for apoptosis detection in vitro. Spas, MZ, and LS set-up, performed and analyzed CAIX ELISA. JAFD and JCS wrote the main manuscript text. JAFD, JCS and KvK prepared the figures. All authors reviewed and approved the manuscript.

\section{Competing interests}

The authors declare no competing interests. 


\section{3 | CAIX in atherosclerosis}

\section{References}

1. Sluimer JC, Gasc JM, van Wanroij JL, et al. Hypoxia, hypoxia-inducible transcription factor, and macrophages in human atherosclerotic plaques are correlated with intraplaque angiogenesis. J Am Coll Cardiol. 2008;51:1258-65.

2. van der Valk FM, Sluimer JC, Voo SA, et al. In Vivo Imaging of Hypoxia in Atherosclerotic Plaques in Humans. JACC Cardiovasc Imaging. 2015;8:1340-1.

3. Marsch E, Theelen TL, Demandt JA, et al. Reversal of hypoxia in murine atherosclerosis prevents necrotic core expansion by enhancing efferocytosis. Arterioscler Thromb Vasc Biol. 2014;34:2545-53.

4. Zatovicova M, Sedlakova O, Svastova E, et al. Ectodomain shedding of the hypoxiainduced carbonic anhydrase IX is a metalloprotease-dependent process regulated by TACE/ADAM17. Br J Cancer. 2005;93:1267-76.

5. Pastorekova S, Parkkila S, Parkkila AK, et al. Carbonic anhydrase IX, MN/CA IX: analysis of stomach complementary DNA sequence and expression in human and rat alimentary tracts. Gastroenterology. 1997;112:398-408.

6. Saarnio J, Parkkila S, Parkkila AK, et al. Immunohistochemistry of carbonic anhydrase isozyme IX (MN/CA IX) in human gut reveals polarized expression in the epithelial cells with the highest proliferative capacity. J Histochem Cytochem. 1998;46:497-504.

7. Neri D and Supuran CT. Interfering with $\mathrm{pH}$ regulation in tumours as a therapeutic strategy. Nat Rev Drug Discov. 2011;10:767-77.

8. Supuran CT. Carbonic anhydrases: novel therapeutic applications for inhibitors and activators. Nat Rev Drug Discov. 2008;7:168-81.

9. Ward C, Meehan J, Gray M, et al. Carbonic Anhydrase IX (CAIX), Cancer, and Radiation Responsiveness. Metabolites. 2018;8.

10. Stillebroer AB, Mulders PF, Boerman OC, et al. Carbonic anhydrase IX in renal cell carcinoma: implications for prognosis, diagnosis, and therapy. Eur Urol. 2010;58:75-83.

11. Lunt SY and Vander Heiden MG. Aerobic glycolysis: meeting the metabolic requirements of cell proliferation. Annu Rev Cell Dev Biol. 2011;27:441-64.

12. Gatenby RA and Gillies RJ. A microenvironmental model of carcinogenesis. Nat Rev Cancer. 2008;8:56-61.

13. Webb BA, Chimenti $\mathrm{M}$, Jacobson MP, et al. Dysregulated $\mathrm{pH}$ : a perfect storm for cancer progression. Nat Rev Cancer. 2011;11:671-7.

14. Gillies RJ, Robey I and Gatenby RA. Causes and consequences of increased glucose metabolism of cancers. J Nucl Med. 2008;49 Suppl 2:24S-42S.

15. Lee $\mathrm{SH}$, Mclntyre $\mathrm{D}$, Honess $\mathrm{D}$, et al. Carbonic anhydrase IX is a pH-stat that sets an acidic tumour extracellular pH in vivo. Br J Cancer. 2018;119:622-630.

16. Kelly B and O'Neill LA. Metabolic reprogramming in macrophages and dendritic cells in innate immunity. Cell Res. 2015;25:771-84.

17. Palsson-McDermott EM and O'Neill LA. The Warburg effect then and now: from cancer to inflammatory diseases. Bioessays. 2013;35:965-73.

18. Naghavi $M$, John $R$, Naguib $S$, et al. $\mathrm{pH}$ Heterogeneity of human and rabbit atherosclerotic plaques; a new insight into detection of vulnerable plaque. Atherosclerosis. 2002;164:27-35.

19. Park SY and Kim IS. Identification of macrophage genes responsive to extracellular acidification. Inflamm Res. 2013;62:399-406.

106 
20. Oorni K, Rajamaki K, Nguyen SD, et al. Acidification of the intimal fluid: the perfect storm for atherogenesis. J Lipid Res. 2015;56:203-14.

21. Liu $\mathrm{CL}$, Zhang $\mathrm{X}$, Liu J, et al. $\mathrm{Na}(+)-\mathrm{H}(+)$ exchanger 1 determines atherosclerotic lesion acidification and promotes atherogenesis. Nat Commun. 2019;10:3978.

22. Gut MO, Parkkila S, Vernerova Z, et al. Gastric hyperplasia in mice with targeted disruption of the carbonic anhydrase gene Car9. Gastroenterology. 2002;123:1889-903.

23. Leppilampi M, Karttunen TJ, Kivela J, et al. Gastric pit cell hyperplasia and glandular atrophy in carbonic anhydrase IX knockout mice: studies on two strains C57/BL6 and BALB/C. Transgenic Res. 2005;14:655-63.

24. Fontaine MAC, Westra MM, Bot I, et al. Low human and murine Mcl-1 expression leads to a pro-apoptotic plaque phenotype enriched in giant-cells. Sci Rep. 2019;9:14547.

25. van Gisbergen MW, Voets AM, Biemans $\mathrm{R}$, et al. Distinct radiation responses after in vitro mtDNA depletion are potentially related to oxidative stress. PLoS One. 2017;12:e0182508.

26. Rosenberg V, Pastorekova S, Zatovicova M, et al. High serum carbonic anhydrase IX predicts shorter survival in head and neck cancer. Bratis/ Lek Listy. 2016;117:201-4.

27. Sluimer JC, Kisters N, Cleutjens KB, et al. Dead or alive: gene expression profiles of advanced atherosclerotic plaques from autopsy and surgery. Physiol Genomics. 2007;30:335-41.

28. Marsch E, Demandt JA, Theelen TL, et al. Deficiency of the oxygen sensor prolyl hydroxylase 1 attenuates hypercholesterolaemia, atherosclerosis, and hyperglycaemia. Eur Heart J. 2016;37:2993-2997.

29. Cleutjens KB, Faber BC, Rousch M, et al. Noninvasive diagnosis of ruptured peripheral atherosclerotic lesions and myocardial infarction by antibody profiling. $J$ Clin Invest. 2008;118:2979-85.

30. Hellings WE, Moll FL, De Vries JP, et al. Atherosclerotic plaque composition and occurrence of restenosis after carotid endarterectomy. JAMA. 2008;299:547-54.

31. Kruijshoop $M$, Feskens EJ, Blaak EE, et al. Validation of capillary glucose measurements to detect glucose intolerance or type 2 diabetes mellitus in the general population. Clin Chim Acta. 2004;341:33-40.

32. Hertle E, van Greevenbroek MM, Arts IC, et al. Distinct associations of complement C3a and its precursor C3 with atherosclerosis and cardiovascular disease. The CODAM study. Thromb Haemost. 2014;111:1102-11.

33. Peeters SA, Engelen L, Buijs J, et al. Associations between advanced glycation endproducts and matrix metalloproteinases and its inhibitor in individuals with type 1 diabetes. J Diabetes Complications. 2018;32:325-329.

34. Goossens P, Gijbels MJ, Zernecke A, et al. Myeloid type I interferon signaling promotes atherosclerosis by stimulating macrophage recruitment to lesions. Cell Metab. 2010;12:142-53.

35. Virmani R, Kolodgie FD, Burke AP, et al. Lessons from sudden coronary death: a comprehensive morphological classification scheme for atherosclerotic lesions. Arterioscler Thromb Vasc Biol. 2000;20:1262-75.

36. Hertle E, Arts IC, van der Kallen CJ, et al. Distinct Longitudinal Associations of MBL, MASP-1, MASP-2, MASP-3, and MAp44 With Endothelial Dysfunction and Intima-Media 


\section{3 | CAIX in atherosclerosis}

Thickness: The Cohort on Diabetes and Atherosclerosis Maastricht (CODAM) Study. Arterioscler Thromb Vasc Biol. 2016;36:1278-85.

37. Zhu L, Zhao Q, Yang T, et al. Cellular metabolism and macrophage functional polarization. Int Rev Immunol. 2015;34:82-100.

38. Diskin C and Palsson-McDermott EM. Metabolic Modulation in Macrophage Effector Function. Front Immunol. 2018;9:270.

39. Eckert AW, Horter S, Bethmann D, et al. Investigation of the Prognostic Role of Carbonic Anhydrase 9 (CAIX) of the Cellular mRNA/Protein Level or Soluble CAIX Protein in Patients with Oral Squamous Cell Carcinoma. Int J Mol Sci. 2019;20.

40. Ilie M, Mazure NM, Hofman V, et al. High levels of carbonic anhydrase IX in tumour tissue and plasma are biomarkers of poor prognostic in patients with non-small cell lung cancer. Br J Cancer. 2010;102:1627-35.

41. de Kleijn DP, Moll FL, Hellings WE, et al. Local atherosclerotic plaques are a source of prognostic biomarkers for adverse cardiovascular events. Arterioscler Thromb Vasc Biol. 2010;30:612-9.

42. Peeters W, de Kleijn DP, Vink A, et al. Adipocyte fatty acid binding protein in atherosclerotic plaques is associated with local vulnerability and is predictive for the occurrence of adverse cardiovascular events. Eur Heart J. 2011;32:1758-68.

43. Chen XJ, Wu S, Yan RM, et al. The role of the hypoxia-Nrp-1 axis in the activation of $\mathrm{M} 2$-like tumor-associated macrophages in the tumor microenvironment of cervical cancer. Mol Carcinog. 2019;58:388-397.

44. Muenst $S$, Laubli $H$, Soysal SD, et al. The immune system and cancer evasion strategies: therapeutic concepts. J Intern Med. 2016;279:541-62.

45. Chiche J, Ilc K, Laferriere J, et al. Hypoxia-inducible carbonic anhydrase IX and XII promote tumor cell growth by counteracting acidosis through the regulation of the intracellular pH. Cancer Res. 2009;69:358-68.

46. Noonan J, Grassia G, MacRitchie N, et al. A Novel Triple-Cell Two-Dimensional Model to Study Immune-Vascular Interplay in Atherosclerosis. Front Immunol. 2019;10:849. 47. Hilvo M, Rafajova M, Pastorekova S, et al. Expression of carbonic anhydrase IX in mouse tissues. J Histochem Cytochem. 2004;52:1313-22.

48. Chemaly M, McGilligan V, Gibson M, et al. Role of tumour necrosis factor alpha converting enzyme (TACE/ADAM17) and associated proteins in coronary artery disease and cardiac events. Arch Cardiovasc Dis. 2017;110:700-711.

49. Zhang C, Zheng L, Nurnberg J, et al. Cleavage of pro-tumor necrosis factor alpha by ADAM metallopeptidase domain 17: a fluorescence-based protease assay cleaves its natural protein substrate. Anal Biochem. 2014;445:14-9.

50. Oksala N, Levula M, Airla N, et al. ADAM-9, ADAM-15, and ADAM-17 are upregulated in macrophages in advanced human atherosclerotic plaques in aorta and carotid and femoral arteries--Tampere vascular study. Ann Med. 2009;41:279-90.

51. Leppanen O, Bjornheden T, Evaldsson M, et al. ATP depletion in macrophages in the core of advanced rabbit atherosclerotic plaques in vivo. Atherosclerosis. 2006;188:32330 .

52. Pelisek J, Deutsch L, Ansel A, et al. Expression of a metalloproteinase family of ADAMTS in human vulnerable carotid lesions. J Cardiovasc Med (Hagerstown). 2017;18:1018. 
53. Verhoeven BA, Velema E, Schoneveld AH, et al. Athero-express: differential atherosclerotic plaque expression of mRNA and protein in relation to cardiovascular events and patient characteristics. Rationale and design. Eur J Epidemiol. 2004;19:1127-33.

54. Pasarica M, Rood J, Ravussin E, et al. Reduced oxygenation in human obese adipose tissue is associated with impaired insulin suppression of lipolysis. J Clin Endocrinol Metab. 2010;95:4052-5.

55. Goossens $\mathrm{GH}$, Bizzarri A, Venteclef N, et al. Increased adipose tissue oxygen tension in obese compared with lean men is accompanied by insulin resistance, impaired adipose tissue capillarization, and inflammation. Circulation. 2011;124:67-76. 
3 | CAIX in atherosclerosis

\section{Supplemental data}

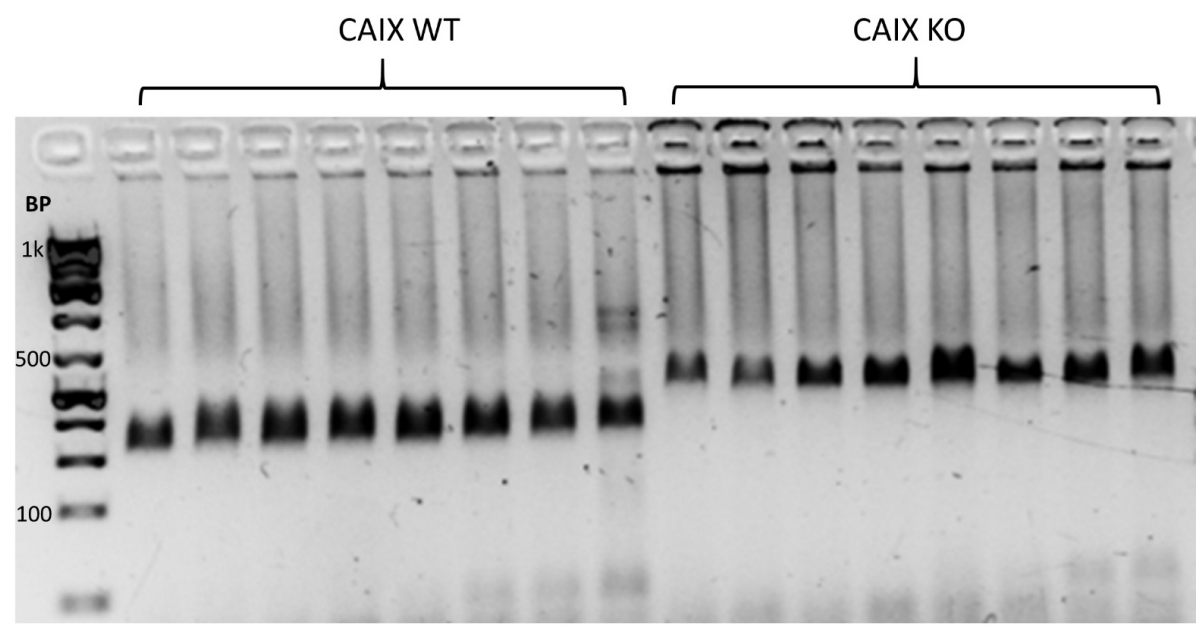

Supplemental figure S1. Genotyping of CAIX DNA in CAIX WT and KO stomach tissue. Expected band WT 318bp, KO 400bp. 

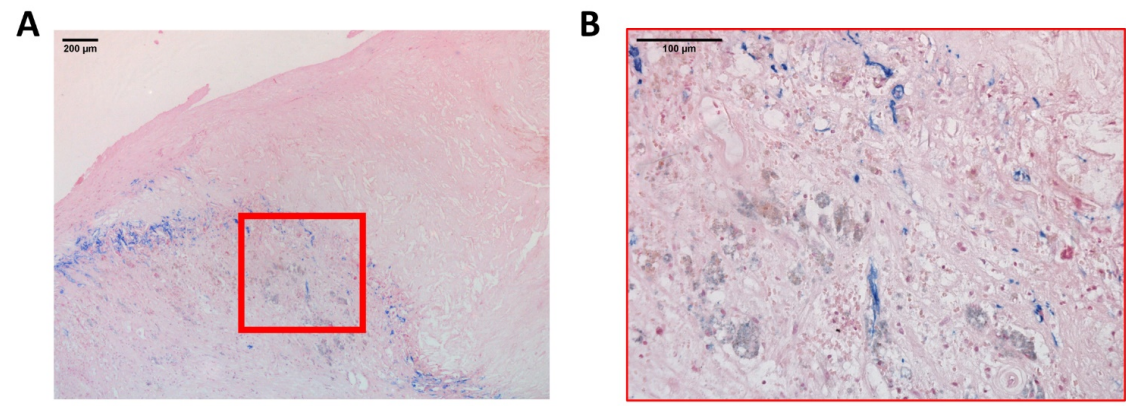

Supplemental Figure S2 Representative immunohistochemical picture of CAIX protein expression in unstable plaque segments of the MaasHPS cohort ( $N=27)$. CAIX in blue, nuclei in red. Depicted as 50x magnification (A) and 200x magnification (B) of the red squared area. 
3 | CAIX in atherosclerosis
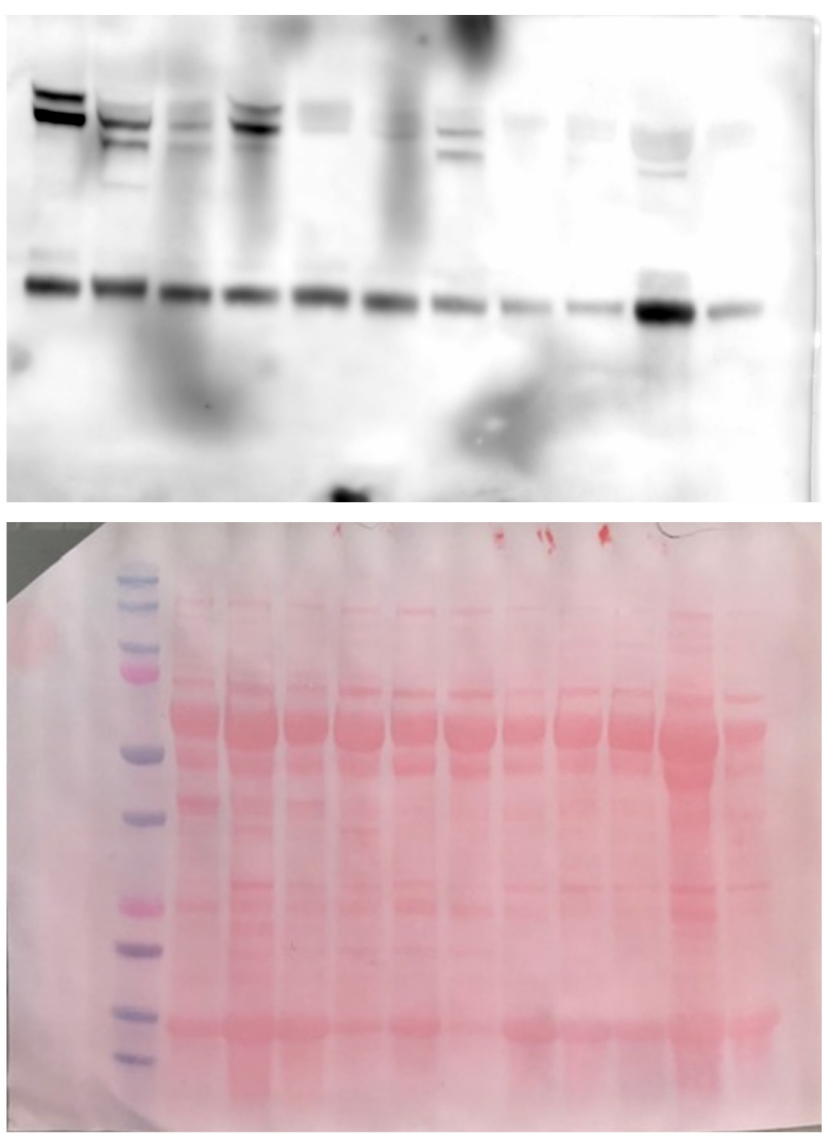

Supplemental figure S3. Full western blots and Ponceau S for Figure 4B 
CAIX in atherosclerosis | $\mathbf{3}$

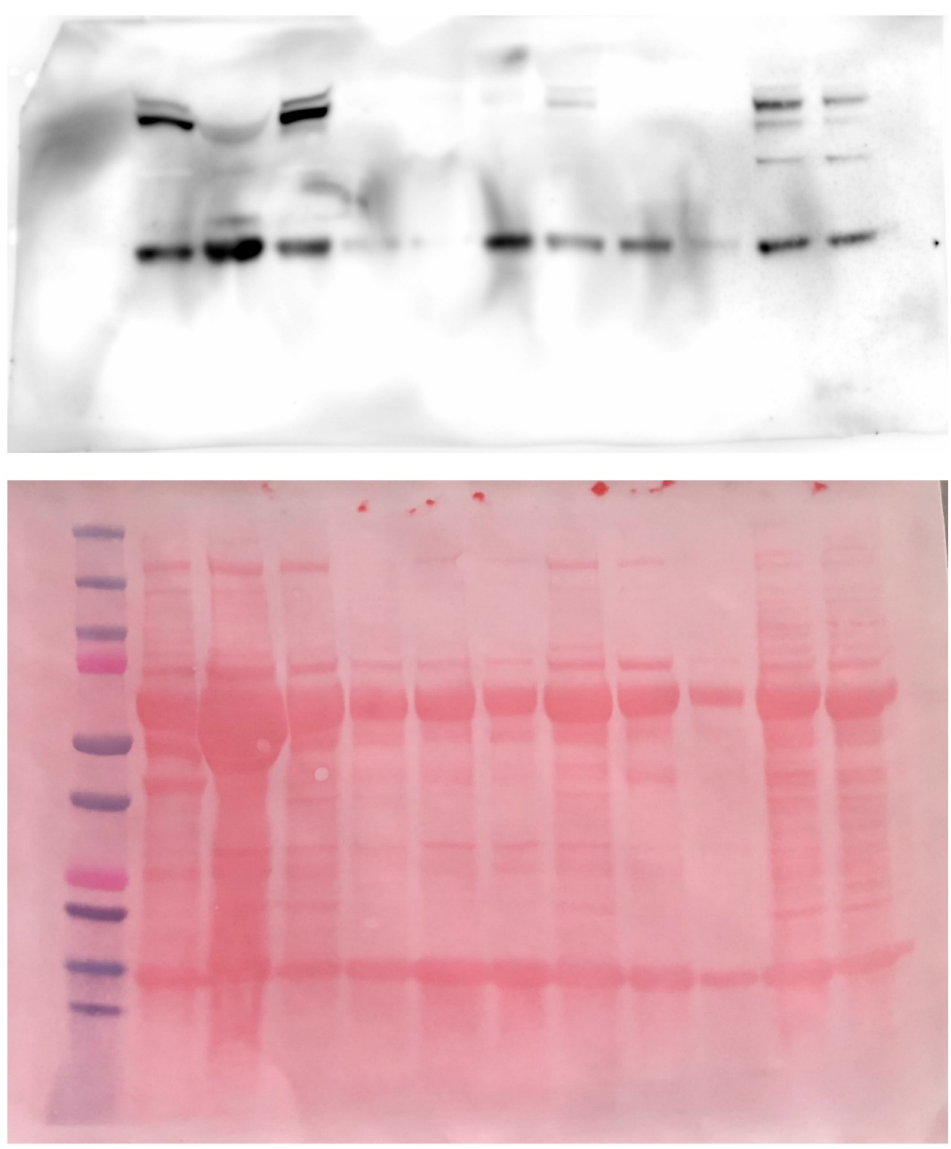

Supplemental Figure S4. Full western blots and Ponceau S for Figure 4D 


\section{3 | CAIX in atherosclerosis}

Supplemental Table 1. Primer sequences for qRT-PCR

\begin{tabular}{|l|l|l|}
\hline Primer & $\mathbf{F W}$ & $\mathbf{R V}$ \\
\hline $\mathbf{1 8 5}$ & GTAACCCGTTGAACCCCATT & CCATCCAATCGGTAGTAGCG \\
\hline Cyclophilin & CAAATGCTGGACCAAACACAA & TTCACCTTCCCAAAGACCACAT \\
\hline iNos & CCTGGTACGGGCATTGCT & GCTCATGCGGCCTCCTTT \\
\hline IL6 & CTGCAAGAGACTTCCATCCAGTT & GAAGTAGGGAAGGCCGTGG \\
\hline TNF & CATCTTCTCAAAATTCGAGTGACAA & TGGGAGTAGACAAGGTACAACCC \\
\hline A20 & CAAGGGCTTTTGCACTCTATGTT & GGCACGGGACATTGTTCTG \\
\hline IL10 & TTTGAATTCCCTGGGTGAGAA & CTCCACTGCCTTGCTCTTATTTTC \\
\hline CD206 & TGCAAAGGACTGAAAGGAAACC & CCAGTCCAGGCATTGAAAGTG \\
\hline
\end{tabular}

Primer sequences used for qRT-PCR. FW forward, RV reverse 
Supplemental Table S2. Correlation CAIX mRNA to apoptosis pathway (MSigDB_HALLMARK_APOPTOSIS) in unstable plaque segments

\begin{tabular}{|c|c|c|}
\hline Gene & Correlation & p.value \\
\hline ENO2 & 0.58 & 0.002 \\
\hline IL1B & 0.57 & 0.002 \\
\hline CDK2 & 0.42 & 0.027 \\
\hline GADD45B & 0.43 & 0.024 \\
\hline GNA15 & 0.43 & 0.025 \\
\hline PEA15 & 0.44 & 0.023 \\
\hline PPP3R1 & 0.45 & 0.017 \\
\hline PTK2 & -0.42 & 0.031 \\
\hline TXNIP & 0.42 & 0.031 \\
\hline CD14 & 0.40 & 0.041 \\
\hline BCL2L1 & 0.28 & 0.159 \\
\hline BTG3 & 0.36 & 0.067 \\
\hline CAV1 & -0.31 & 0.117 \\
\hline CDKN1A & 0.31 & 0.113 \\
\hline CTH & -0.31 & 0.113 \\
\hline DFFA & -0.32 & 0.108 \\
\hline DNAJA1 & -0.36 & 0.063 \\
\hline EREG & -0.30 & 0.122 \\
\hline GSTM1 & -0.29 & 0.149 \\
\hline HMGB2 & 0.31 & 0.112 \\
\hline IL1A & 0.34 & 0.081 \\
\hline NEDD9 & 0.31 & 0.120 \\
\hline NEFH & 0.29 & 0.149 \\
\hline PDGFRB & -0.31 & 0.115 \\
\hline PLCB2 & 0.31 & 0.111 \\
\hline RELA & 0.28 & 0.156 \\
\hline ROCK1 & -0.34 & 0.080 \\
\hline SLC20A1 & 0.30 & 0.134 \\
\hline SOD1 & -0.37 & 0.056 \\
\hline TGFBR3 & -0.31 & 0.112 \\
\hline TIMP1 & -0.28 & 0.159 \\
\hline TIMP2 & -0.29 & 0.135 \\
\hline VDAC2 & -0.30 & 0.135 \\
\hline BNIP3L & 0.27 & 0.172 \\
\hline CD44 & 0.27 & 0.178 \\
\hline PLAT & 0.27 & 0.175 \\
\hline BMP2 & -0.26 & 0.193 \\
\hline IFNGR1 & 0.26 & 0.193 \\
\hline
\end{tabular}


3 | CAIX in atherosclerosis

\begin{tabular}{|c|c|c|}
\hline PAK1 & 0.24 & 0.228 \\
\hline RHOT2 & 0.24 & 0.234 \\
\hline AIFM3 & 0.15 & 0.445 \\
\hline ANKH & -0.20 & 0.327 \\
\hline ANXA1 & -0.21 & 0.291 \\
\hline APP & 0.10 & 0.625 \\
\hline ATF3 & -0.20 & 0.306 \\
\hline AVPR1A & 0.09 & 0.641 \\
\hline BAX & 0.10 & 0.612 \\
\hline BCL10 & -0.12 & 0.546 \\
\hline BCL2L10 & -0.18 & 0.371 \\
\hline BCL2L11 & -0.09 & 0.662 \\
\hline BCL2L2 & 0.09 & 0.656 \\
\hline BGN & 0.06 & 0.753 \\
\hline BIK & 0.06 & 0.768 \\
\hline BIRC3 & 0.14 & 0.486 \\
\hline BTG2 & -0.15 & 0.462 \\
\hline CASP1 & 0.07 & 0.742 \\
\hline CASP3 & 0.09 & 0.671 \\
\hline CASP4 & 0.08 & 0.700 \\
\hline CASP6 & -0.16 & 0.436 \\
\hline CASP7 & -0.08 & 0.679 \\
\hline CASP8 & -0.17 & 0.392 \\
\hline CASP9 & 0.07 & 0.714 \\
\hline CCND2 & 0.22 & 0.269 \\
\hline CD2 & 0.07 & 0.734 \\
\hline CD38 & -0.15 & 0.444 \\
\hline CD69 & 0.11 & 0.571 \\
\hline CDC25B & 0.14 & 0.499 \\
\hline CDKN1B & -0.10 & 0.613 \\
\hline CREBBP & -0.16 & 0.439 \\
\hline DAP & -0.08 & 0.700 \\
\hline DAP3 & -0.06 & 0.750 \\
\hline DCN & 0.08 & 0.692 \\
\hline DDIT3 & -0.06 & 0.763 \\
\hline DNAJC3 & -0.15 & 0.451 \\
\hline DPYD & -0.09 & 0.649 \\
\hline EGR3 & -0.07 & 0.718 \\
\hline ERBB2 & -0.11 & 0.590 \\
\hline ERBB3 & 0.09 & 0.640 \\
\hline $\mathbf{F 2}$ & -0.14 & 0.491 \\
\hline FAS & -0.11 & 0.599 \\
\hline
\end{tabular}




\begin{tabular}{|c|c|c|}
\hline FASLG & 0.09 & 0.671 \\
\hline FDXR & 0.15 & 0.468 \\
\hline FEZ1 & -0.17 & 0.397 \\
\hline GADD45A & -0.08 & 0.686 \\
\hline GCH1 & 0.08 & 0.680 \\
\hline GPX1 & 0.14 & 0.491 \\
\hline GPX3 & 0.19 & 0.340 \\
\hline GPX4 & -0.14 & 0.493 \\
\hline GSN & 0.09 & 0.672 \\
\hline GSR & 0.09 & 0.668 \\
\hline GUCY2D & -0.22 & 0.262 \\
\hline HGF & -0.19 & 0.345 \\
\hline HMOX1 & -0.14 & 0.477 \\
\hline HSPB1 & -0.21 & 0.299 \\
\hline IFNB1 & 0.13 & 0.515 \\
\hline IGF2R & -0.09 & 0.671 \\
\hline IGFBP6 & -0.08 & 0.693 \\
\hline IL6 & 0.15 & 0.440 \\
\hline IRF1 & -0.10 & 0.604 \\
\hline JUN & -0.08 & 0.702 \\
\hline LEF1 & -0.08 & 0.685 \\
\hline LGALS3 & -0.17 & 0.385 \\
\hline LMNA & -0.16 & 0.414 \\
\hline LUM & 0.19 & 0.331 \\
\hline MCL1 & 0.08 & 0.700 \\
\hline PPP2R5B & 0.20 & 0.324 \\
\hline PPT1 & 0.10 & 0.607 \\
\hline PRF1 & 0.13 & 0.534 \\
\hline PSEN2 & -0.21 & 0.285 \\
\hline RARA & 0.07 & 0.741 \\
\hline RETSAT & 0.12 & 0.537 \\
\hline RHOB & 0.08 & 0.684 \\
\hline RNASEL & 0.10 & 0.622 \\
\hline SMAD7 & -0.19 & 0.338 \\
\hline SOD2 & 0.06 & 0.767 \\
\hline SQSTM1 & -0.14 & 0.498 \\
\hline TAP1 & 0.06 & 0.759 \\
\hline TGFB2 & -0.16 & 0.414 \\
\hline TIMP3 & -0.14 & 0.493 \\
\hline TNF & 0.19 & 0.347 \\
\hline TOP2A & 0.09 & 0.650 \\
\hline TSPO & -0.12 & 0.567 \\
\hline & & \\
\hline & & \\
\hline
\end{tabular}


3 | CAIX in atherosclerosis

\begin{tabular}{|c|c|c|}
\hline WEE1 & -0.19 & 0.332 \\
\hline CLU & -0.05 & 0.794 \\
\hline H1F0 & 0.05 & 0.796 \\
\hline PSEN1 & 0.05 & 0.793 \\
\hline ADD1 & 0.01 & 0.972 \\
\hline BCAP31 & -0.04 & 0.851 \\
\hline BID & 0.00 & 0.989 \\
\hline BMF & 0.04 & 0.860 \\
\hline BRCA1 & -8.10 & 1.000 \\
\hline CASP2 & -0.02 & 0.938 \\
\hline CCNA1 & 0.00 & 0.998 \\
\hline CCND1 & -0.03 & 0.891 \\
\hline CFLAR & -0.01 & 0.961 \\
\hline CTNNB1 & -0.01 & 0.941 \\
\hline CYLD & -0.02 & 0.936 \\
\hline DIABLO & -0.01 & 0.968 \\
\hline DNM1L & 0.00 & 0.992 \\
\hline EBP & -0.04 & 0.844 \\
\hline EMP1 & 0.03 & 0.896 \\
\hline ETF1 & -0.02 & 0.915 \\
\hline F2R & 0.03 & 0.888 \\
\hline IER3 & 0.00 & 0.994 \\
\hline IFITM3 & 0.00 & 0.984 \\
\hline IL18 & 0.01 & 0.941 \\
\hline ISG20 & 0.04 & 0.832 \\
\hline KRT18 & 0.05 & 0.818 \\
\hline MADD & -0.05 & 0.818 \\
\hline MGMT & 0.02 & 0.941 \\
\hline MMP2 & -0.01 & 0.956 \\
\hline PDCD4 & 0.00 & 0.996 \\
\hline PMAIP1 & 0.01 & 0.957 \\
\hline SAT1 & 0.01 & 0.966 \\
\hline SATB1 & 0.04 & 0.838 \\
\hline SPTAN1 & 0.04 & 0.856 \\
\hline TNFRSF12A & 0.00 & 0.986 \\
\hline TNFSF10 & 0.03 & 0.874 \\
\hline
\end{tabular}


Supplemental Table S3. Athero-Express patient demographics and plaque traits in subjects with and without detectable plaque-resident CAIX

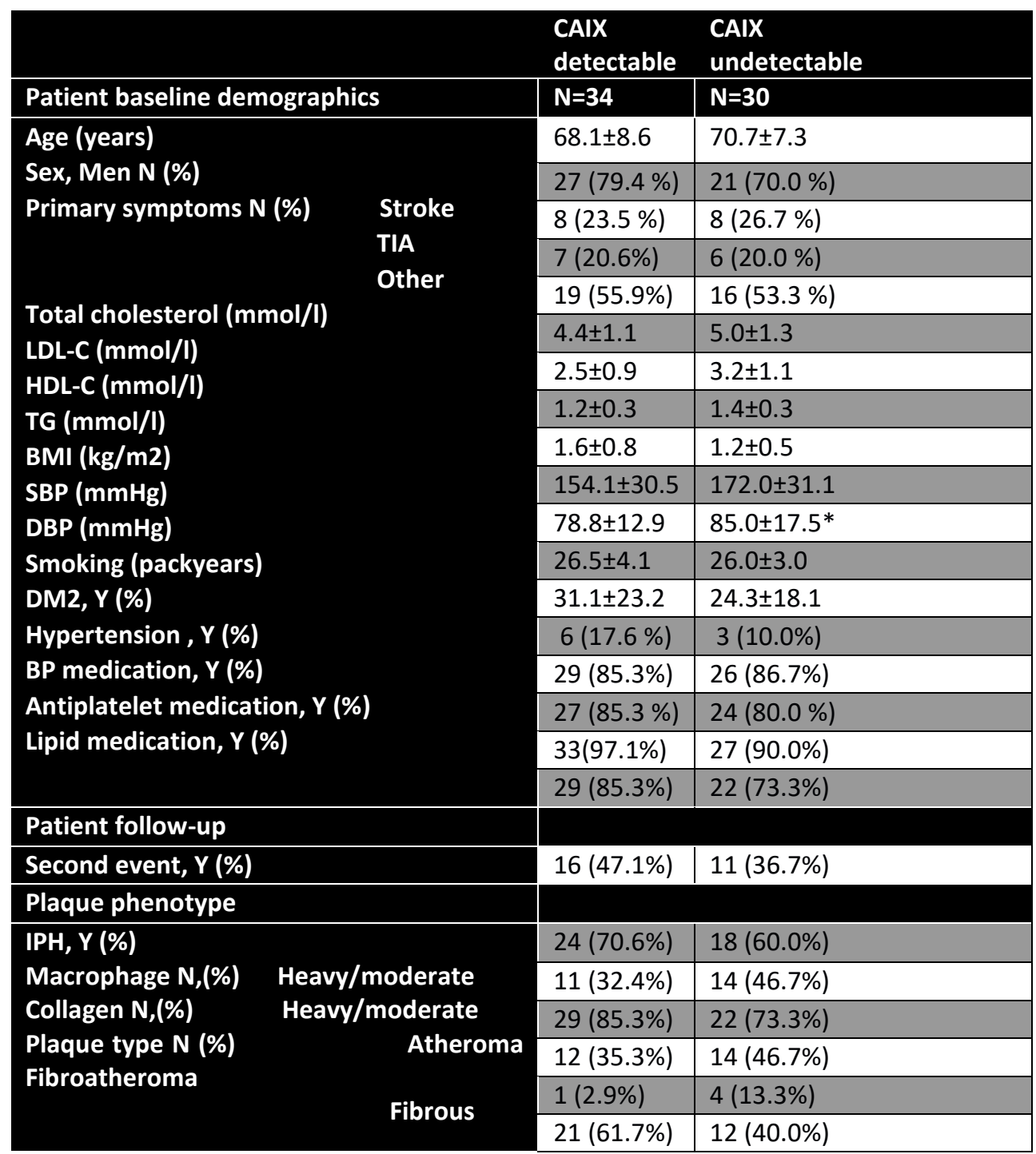

Data are presented as mean \pm SD or proportion (\%, categorical variables). BMI; body mass index. SBP; systolic blood pressure. DBP; diastolic blood pressure. LDL-C; low-density lipoprotein cholesterol, HDLC; high-density lipoprotein cholesterol. TG; triglycerides. DM2; diabetes mellitus type 2. Collagen/macrophage categories Heavy-moderate vs no-minor. Data were analyzed using Mann Whitney rank sum or Fisher's Exact test, and corrected for multiple testing. $Y$, counts for category "yes", N counts. * P-value $<0.05$, all others not significant. 
3 | CAIX in atherosclerosis

Supplemental Table S4. Patient demographics and exploratory data of CODAM cohort

\begin{tabular}{|c|c|c|c|}
\hline & $\begin{array}{l}\text { sCAIX } \\
\text { detectable }\end{array}$ & $\begin{array}{l}\text { sCAIX } \\
\text { undetectable }\end{array}$ & P-value \\
\hline General/metabolic measurements & $\mathrm{N}=\mathbf{8 0}$ & $\mathrm{N}=492$ & \\
\hline Age (years) & $60.9 \pm 6.9$ & $59.4 \pm 6.9$ & 0.085 \\
\hline Sex, N Men (\%) & $47(58.8 \%)$ & $304(61.8 \%)$ & 0.605 \\
\hline BMI (kg/m2) & $28.6 \pm 4.7$ & $28.6 \pm 4.2$ & 0.872 \\
\hline Waist $(\mathrm{cm})$ & $99.2 \pm 12.0$ & $99.3 \pm 11.9$ & 0.948 \\
\hline SBP (mmHg) & $141.4 \pm 19.5$ & $140.2 \pm 19.3$ & 0.618 \\
\hline DBP (mmHg) & $81.8 \pm 9.5$ & $81.9 \pm 9.3$ & 0.942 \\
\hline Fasting Plasma Glucose (mmol/l) & $6.3 \pm 1.8$ & $6.0 \pm 1.4$ & 0.214 \\
\hline Total cholesterol (mmol/l) & $5.1 \pm 1.0$ & $5.2 \pm 1.0$ & 0.407 \\
\hline HDL-C (mmol/l) & $1.21 \pm 0.35$ & $1.18 \pm 0.35$ & 0.650 \\
\hline TG (mmol/l) & $1.4[0.9 ; 1.8]$ & $1.4[1.0 ; 2.0]$ & 0.085 \\
\hline Current smoker, Y (\%) & $13(16.2 \%)$ & $101(21.1 \%)$ & 0.320 \\
\hline Packyears* & $5.3[0 ; 30.6]$ & $15.0[0 ; 31.1]$ & 0.053 \\
\hline \multirow{2}{*}{$\begin{array}{l}\text { Smoking, Current/Former/Never } \\
\text { (\%) }\end{array}$} & $13 / 32 / 35$ & $101 / 251 / 127$ & \multirow[t]{2}{*}{0.007} \\
\hline & $(16.2 / 40.0 / 43.8)$ & $(21.1 / 2.4 / 26.5)$ & \\
\hline \multirow{2}{*}{$\begin{array}{l}\text { NGM/IGM/DM2 } \\
\text { (\%) }\end{array}$} & $47 / 10 / 23$ & $252 / 117 / 123$ & \multirow[t]{2}{*}{0.079} \\
\hline & $(58.8 / 12.5 / 28.7)$ & $(51.2 / 23.8 / 25.0)$ & \\
\hline DM2, Y (\%) & $23(28.7 \%)$ & $123(25.0 \%)$ & 0.476 \\
\hline Glucose medication, Y (\%) & $18(22.5 \%)$ & $58(11.8 \%)$ & 0.009 \\
\hline Lipid medication, Y (\%) & $11(10.2 \%)$ & $97(19.7 \%)$ & 0.206 \\
\hline BP medication, Y (\%) & $23(28.7 \%)$ & $197(40.0 \%)$ & 0.054 \\
\hline Rheumatoid arthritis, Y (\%) & $7(9.1 \%)$ & $43(8.8 \%)$ & 0.944 \\
\hline Cancer, Y (\%) & $3(15.0 \%)$ & $17(3.5 \%)$ & 0.905 \\
\hline \multicolumn{4}{|l|}{ Cardiovascular measurements } \\
\hline CVD, Y (\%) & $20(25.0 \%)$ & $138(28.1 \%)$ & 0.565 \\
\hline CVE, Y (\%) & $11(13.8 \%)$ & $78(15.9 \%)$ & 0.625 \\
\hline IMT** & $0.80 \pm 0.19$ & $0.77 \pm 0.15$ & 0.140 \\
\hline AAlx $* * *$ & $1.09 \pm 0.13$ & $1.10 \pm 0.13$ & 0.548 \\
\hline PAD (=AAix< <.9) , Y (\%) & $5(6.2 \%)$ & $23(4.7 \%)$ & 0.548 \\
\hline Plaque present, Y (\%) & \multirow[t]{2}{*}{$3(6.7 \%)$} & \multirow[t]{2}{*}{$14(4.4 \%)$} & \multirow[t]{2}{*}{0.491} \\
\hline (available for only 366) & & & \\
\hline \multicolumn{4}{|l|}{ Other measurements } \\
\hline MMP2 (ng/ml) & $157[141 ; 177]$ & $146[129 ; 163]$ & $<0.001$ \\
\hline MMP9 (ng/ml) & $14.3[10.7 ; 20.1]$ & $14.5[11.4 ; 20.1]$ & 0.448 \\
\hline IL6 (ng/l) & $1.6[1.1 ; 2.5]$ & $1.6[1.2 ; 2.2]$ & 0.959 \\
\hline eGFR ( $\mathrm{ml} 1.73 \mathrm{~m} 2 / \mathrm{min})$ & $90.7[80.3 ; 100.2]$ & $\begin{array}{l}90.5 \\
{[78.1 ; 101.3]}\end{array}$ & 0.775 \\
\hline
\end{tabular}


* Values for packyears were analyzed in 560 participants. **Values for cIMT were analyzed in 504 participants. **Values for AAlx were analyzed in 541 participants. Data are presented as mean \pm SD (normally distributed variables), median [interquartile range] (skewed variables) or proportion (\%, categorical variables). BMI; body mass index. SBP; systolic blood pressure. DBP; diastolic blood pressure. HDL-C; high-density lipoprotein cholesterol. TG; triglycerides. NGM; normal glucose metabolism. IGM; impaired glucose metabolism. DM2; diabetes mellitus type 2. CVD; cardiovascular disease. CVE; cardiovascular event. IMT; intima-media thickness. AAlx; ankle-arm index. PAD; peripheral artery disease. MMP2/9; matrix metalloproteinase 2/9. IL6; interleukin 6. eGFR; estimated glomerular filtration rate. $\mathrm{Y}$, counts for yes. $\mathrm{p}$-Values were obtained by ANOVA, ANOVA of Intransformed variables or Pearson Chi-square.

\section{Supplemental Table S5. Association of SCAIX with incident CVD and CVE}

\begin{tabular}{|l|l|l|l|}
\hline CVD & \multicolumn{1}{l}{ OR } & \multicolumn{1}{l}{ 95\%; Cl } & p-value \\
\hline Model 1 & 0.90 & $0.410 ; 1.965$ & 0.787 \\
\hline Model 2 & 0.87 & $0.393 ; 1.904$ & 0.719 \\
\hline Model 3 & 0.82 & $0.359 ; 1.878$ & 0.640 \\
\hline CVE & OR & 95\%; Cl & p-value \\
\hline Model 1 & 0.62 & $0.233 ; 1.629$ & 0.329 \\
\hline Model 2 & 0.59 & $0.223 ; 1.580$ & 0.296 \\
\hline Model 3 & 0.63 & $0.229 ; 1.737$ & 0.373 \\
\hline
\end{tabular}

Logistic regression analysis using 317 and 369 subjects that were free of CVD or CVE at time of inclusion, respectively. sCAIX was treated as dichotomous independent variable (detectable yes/no). $\beta$ indicates mutation of dependent variable if CAIX is detectable (yes). Model 1: Crude, no adjustments. Model 2: model $1+$ adjustment for sex and age. Model 3: model $2+$ adjustments for smoking (status [current, former, never] \& packyears), medication (lipid-modifying $y / n$, anti-HT y/n, glucose-lowering $\mathrm{y} / \mathrm{n}$ ), glucose metabolism status (IGM y/n, DM2 y/n). 



\section{Chapter 4}

Heterogeneity and plasticity in healthy and atherosclerotic vasculature explored by single cell sequencing

Van Kuijk K, Kuppe C, Betsholtz C, Vanlandewijck M, Kramann R, Sluimer JC

Cardiovascular Research 2019 Oct 1;115(12):1705-1715 



\begin{abstract}
Cellular characteristics and their adjustment to a state of disease have become more evident due to recent advances in imaging, fluorescent reporter mice, and whole genome RNA sequencing. The uncovered cellular heterogeneity and/or plasticity potentially complicates experimental studies and clinical applications, as markers derived from whole tissue 'bulk' sequencing is unable to yield a subtype transcriptome and specific markers. Here, we propose definitions on heterogeneity and plasticity, discuss current knowledge thereof in the vasculature and how this may be improved by single-cell sequencing (SCS). SCS is emerging as an emerging technique, enabling researchers to investigate different cell populations in more depth than ever before. Cell selection methods, e.g. flow assisted cell sorting, and the quantity of cells can influence the choice of SCS method. Smart-Seq2 offers sequencing of the complete mRNA molecule on a low quantity of cells, while Drop-seq is possible on large numbers of cells on a more superficial level. SCS has given more insight in heterogeneity in healthy vasculature, where it revealed that zonation is crucial in gene expression profiles among the anatomical axis. In diseased vasculature, this heterogeneity seems even more prominent with discovery of new immune subsets in atherosclerosis as proof. Vascular smooth muscle cells and mesenchymal cells also share these plastic characteristics with the ability to up-regulate markers linked to stem cells, such as Sca-1 or CD34. Current SCS studies show some limitations to the number of replicates, quantity of cells used, or the loss of spatial information. Bioinformatical tools could give some more insight in current datasets, making use of pseudo-time analysis or RNA velocity to investigate cell differentiation or polarization. In this review, we discuss the use of SCS in unravelling heterogeneity in the vasculature, its current limitations and promising future applications.
\end{abstract}




\section{4 | Heterogeneity and plasticity in atherosclerosis}

Atherosclerosis is a long process of lipid and inflammatory cell accumulation in the vessel wall, leading to plaque formation and ultimately plaque rupture. Clinical manifestations of cardiovascular diseases are still the leading cause of death worldwide, necessitating better, targeted treatment. ${ }^{1}$ Current therapies to reduce the clinical manifestations, myocardial infarction and stroke, have been aimed at one or multiple risk factors such as dyslipidemia, hypertension or inflammation..$^{2-4}$ However, as many cell types are involved and/or dysfunctional, a fully effective therapy has not been developed. Pinpointing progression of a disease to a certain cell type is challenging because of strong heterogeneity and/or plasticity of cells not only inside the plaque, but also the surrounding tissue. Hence, we see the need to define and address heterogeneity in the healthy and atherosclerotic vasculature, and highlight a new technology to capture this heterogeneity at an unprecedented level: single cell sequencing.

Before discussing cellular heterogeneity and plasticity in detail, one has to consider the classical definition of a cell type. The distinction between classical vascular cell types, such as endothelial cells (ECs), smooth muscle cells (SMCs), macrophages, and fibroblasts, is based on embryonic germ line origin, anatomical or organ location, microscopic morphology and phenotype/function. In the distant past, this was largely based on morphology, while it is currently also based on population averages of the transcriptome, the expression of classical cell type markers, i.e cluster of differentiation (CD) molecules, and lineage reporters models using these classical markers. In this framework, one can distinguish differences within a cell type, and changes between cell types, i.e. heterogeneity and plasticity. These terms are used interchangeably, causing considerable confusion. Much knowledge can be gained from the stem cell field, where cell plasticity and heterogeneity are often discussed subjects. The presence of absence of marker genes linked to stem cells, for instance stem cell antigen-1 (Sca-1) or CD34, does not limit the cell in question as belonging to the stem cell population. ${ }^{5,6}$ It rather shows that a range of genes are linked to stem cells and the expression of these genes is possibly different between cells in this population, creating a very heterogeneous cell population. Therefore, in this review, we will describe heterogeneity as moderate changes in transcriptome and function, enabling adaptation to the micro-environment, organ or anatomical location. Importantly, this adaptation does not lead in loss or acquisition of classical cell identity markers and yields cellular subtypes. Often adaptation of a cell to its environment is also termed phenotypic plasticity, but if cell identity is not lost, we regard this as heterogeneity. Cellular plasticity, on the other hand, is used here to refer to complete changes in cell identity, upon changes in micro-environment. This process is accompanied by loss or acquisition of classical cell identity markers, and includes so called trans-differentiation and reversal of this. Taken together, plasticity and heterogeneity may be regarded as cell types versus subtypes. A schematic overview of vascular cell types and their heterogeneous phenotypes is depicted in Figure 1. Certainly, this definition is not always unambiguous, and we will discuss the potential benefit of SCS to aid in this distinction. 


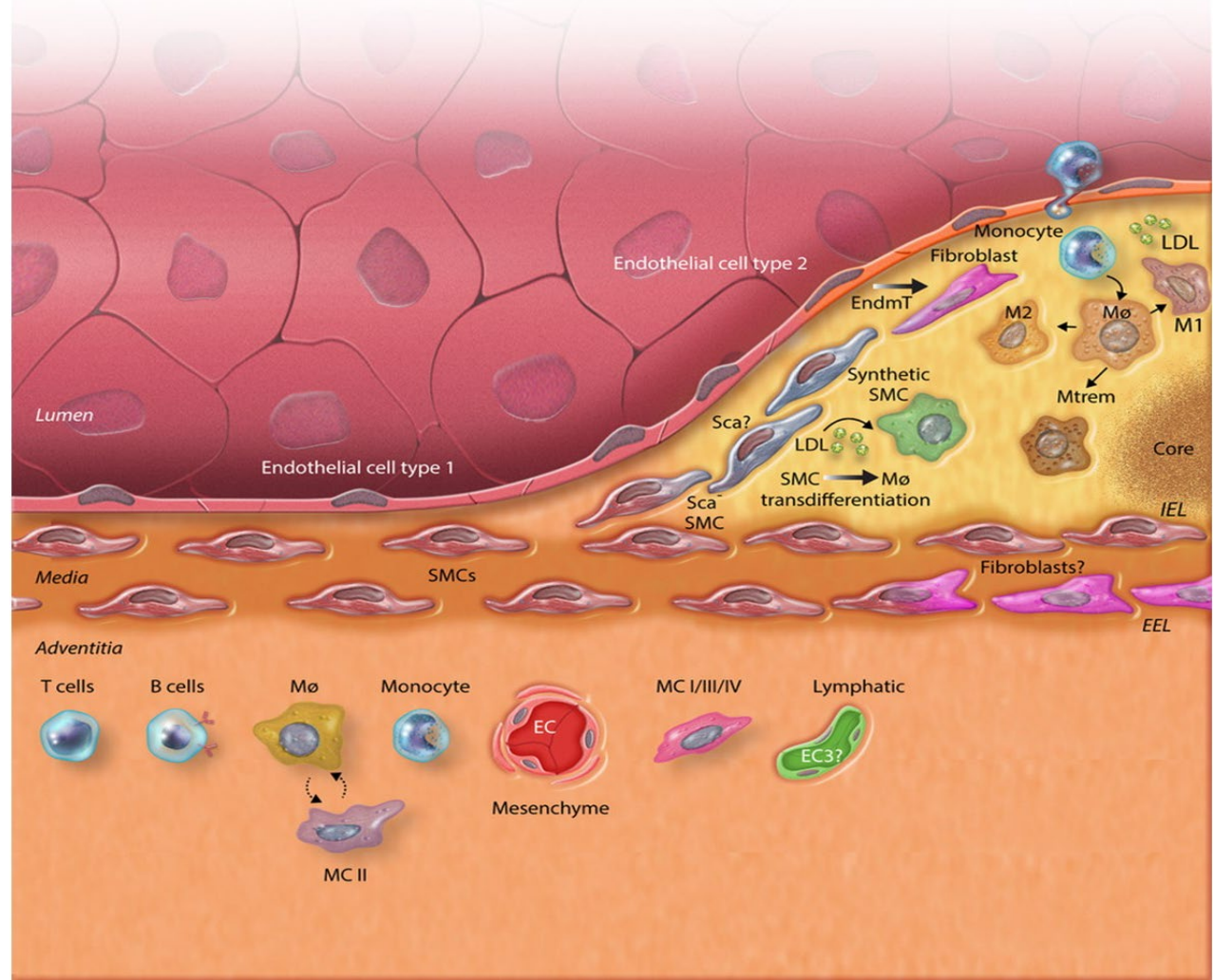

Figure 1. The different layers of the vasculature (adventitia, media, and intima) and the development of atherosclerosis with all involved cell types. The graphical overview shows heterogeneity (indicated here by thick, black/white filled arrows and cell types in distinct colors) and plasticity (indicated here by single line, black arrows, and cell types in shades of the same color) of all these subsets and their capability to adjust their phenotype to the lipid-rich environment. Endothelial cell (EC) types are zonated 46,55 and EC I and II can undergo endothelial-to-mesenchymal transition (EndMT) in hyperlipidemia. Smooth muscle cells (SMCs) can translocate to the cap and become more synthetic. Moreover, they can transdifferentiate into a macrophage-like cell upon lipid engulfing. Macrophages $(\mathrm{Mu})$ are depicted with their different subsets according to certain gene expression profiles (M1, M2, MTrem ${ }^{61}$ ). They are located in the lipid-rich intima, just above the interna elastica lamina (IEL). In the adventitia, located underneath the externa elastica lamina (EEL), several mesenchymal subsets appear, indicated with I-II-III-IV. ${ }^{56}$ The adventitia is mostly inhabited by these subsets of mesenchymal cells (MCs), immune cells, and distinct EC subsets. These different subsets all have different functional profiles. Macrophages and MC II were shown to cross-talk as indicated by dotted arrow. The ? indicates new findings or unclarities that need further study. 


\section{4 | Heterogeneity and plasticity in atherosclerosis}

\section{Heterogeneity and plasticity in the vasculature: current evidence}

The process of atherosclerosis starts with dysfunction of the endothelial cells facing the vascular lumen. This leads to the extravasation of low density lipoprotein (LDL) into the subendothelial space. Here LDL accumulates, is oxidized and will further trigger inflammation. ${ }^{7,8}$ Monocytes are attracted by inflammatory cytokines originating from the forming fatty streak and try to phagocytose the growing amount of LDL, leading to the formation of foamy macrophages. After extensive LDL uptake, these macrophages go into apoptosis and are cleared by other macrophages through efferocytosis. ${ }^{9}$ However, when plaque development progresses, the amount of apoptotic cells increases and clearance by other macrophages becomes ineffective. Post-apoptotic necrosis occurs, leading to the formation of a necrotic core in the plaque consisting of dead cells and cholesterol crystals. Macrophages release tissue factor, matrix proteases and pro-angiogenic factors, which influence plaque stability and ultimately plaque rupture. ${ }^{10,11}$ Alongside the growing amount of macrophages and thus growing necrotic core, the amount of alpha smooth muscle actin (aSMA)+ smooth muscle cells (SMCs) lining the atherosclerotic plaque will diminish. The role of SMCs in atherosclerosis is already marked at the very beginning of the process, when intimal thickening is observed due to hemodynamic shear stress. Matrix proteoglycans, collagen and elastin fibers are secreted and a stable environment is created. ${ }^{7}$ While the plaque grows, SMCs migrate from the medial layer towards the lumen forming the fibrous cap. This fibrous cap becomes thinner over the years due to SMC apoptosis and matrix degradation by macrophages. This can ultimately lead to cap rupture, exposing the plaques' thrombogenic content, triggering thrombus formation and lumen occlusion, and consequently causing myocardial infarction or stroke.

In recent years evidence accumulates that most of the major cell types in atherosclerotic plaques, e.g. endothelial cells, macrophages, T-cells and SMCs are heterogeneous and/or plastic to some extent. William Aird highlighted different concepts of endothelial heterogeneity in atherosclerosis with regards to anatomical location, activation and dysfunction. ${ }^{12}$ He stipulated that endothelial cell heterogeneity and plasticity are dependent on multiple factors. The same is true for SMCs, who undergo phenotypic switching upon lipid and cytokine exposure in the plaque. The SMCs switch from a quiescent state to a proliferative, more migrative state, is also known as contractile-to-synthetic switch, which we classify as heterogeneity of subtypes. ${ }^{13}$ Lipid loading does not solely trigger contractile-to-synthetic switch, but also initiates trans-differentiation to macrophage-like smooth muscle cells and may be classified as plasticity of SMCs . Multiple groups have now shown that expression of different macrophage markers, like galectin-3 (LGALS3) and CD68, increased during lipid-loading of SMCs, while the SMC markers alphaactin-2 (ACTA2) and myosin heavy chain 11 (MYH11) decreased in expression. ${ }^{14-18}$ Immune cell heterogeneity is also widely discussed in atherosclerosis development, emphasizing different polarization states of macrophages. Polarization of macrophages into the pro-inflammatory M1 macrophage, via LPS or TNF $\alpha$, versus the anti-inflammatory M2 128 
macrophage, via IL-4 or IL-10, already shows a distinct phenotypic difference. ${ }^{19}$ However, we now know that the range of phenotypes is much more subtle and diverse than $\mathrm{M} 1$ versus $\mathrm{M} 2$ and that stimuli and microenvironment are decisive for every subset of macrophages. ${ }^{20-}$ ${ }^{22}$ Immune cell heterogeneity is not only restricted to macrophages, but also occurs in other immune subsets. Activated macrophages can recruit T-cells, and therefore further enhance inflammation. These T-cells are not only activated by macrophages and their secreted cytokines, but also by the vast amount of oxLDL in the plaque. ${ }^{23}$ This again yields a broad spectrum of differentially activated T-cells. The diversity of macrophages and T-cells opens up the possibility for drugs to tackle small subsets of immune cells with distinct phenotypes regarding plaque progression. ${ }^{24}$

Although endothelial cells, macrophages, T cells and SMCs are the most discussed cells relating to disease progression, recent research in the field has shown that mesenchymal cells possibly also play a role. They may originate from a mesenchymal stem cell-like cell type, which can give rise to various cell types like (myo)fibroblasts or SMCs. ${ }^{25}$ These cells have been reported to stem from the adventitial layer surrounding the vasculature and are positive for stem cell markers, like stem cell antigen 1 (Sca1) and GLI-Kruppel family member 1 (Gli1). ${ }^{26-28}$ Furthermore, evidence suggests that these cells originate from a process called endothelial-to-mesenchymal transition (EndMT), which can be triggered via various pathways. ${ }^{29,} 30$ Endothelial cells exposed to different plaque traits, like hypoxia, oxidative stress or transforming growth factor $\beta$ (TGF- $\beta$ ), undergo this transformation where they lose gene and protein expression of endothelial markers like CD31, endothelial nitric oxide synthase (eNOS) while simultaneously gaining mesenchymal markers such as fibroblast activation protein (FAP), alpha-actin 2 (ACTA2) and regulatory transcription factors Snail and Slug, SNAI1\&2 respectively. ${ }^{29}$ In human atherosclerotic plaques, EndMT is usually found in larger, unstable plaques and thus linking EndMT to plaque instability.

These examples already clearly illustrate the intricate complexity of atherosclerosis development and all cell types involved, with heterogeneity as a key concept. Heterogeneity of SMCS, endothelial cells, mesenchymal cells and immune cells makes it difficult to study them in the context of healthy and diseased vasculature. However, an emerging technique might be able to give us more insight than ever before. The recent advances in the field of single cell sequencing (SCS) are providing an unprecedented opportunity to unravel complex biological systems on multiple biological levels with single cell resolution. The averaged data scientists have generated using bulk populations of cells or whole tissues can obscure relevant biological insight. Moreover, SCS enables researchers to zoom in on cell populations and investigate them in more depth. This potentially yields new cell phenotypes, uncovering subpopulations with different functions, and providing definitive answers to issues of cellular- trans-differentiation. In this review, we will discuss the use of single cell sequencing to unravel heterogeneity in healthy and disease vasculature. We will 


\section{4 | Heterogeneity and plasticity in atherosclerosis}

first summarize the principles and different methods for SCS, followed by discussion of published data on heterogeneity in healthy and diseased vasculature using SCS.

\section{SCS technologies today}

A decade ago, Tang and colleagues reported that it was possible to gain substantial transcriptomic information out of a single cell using next generation sequencing. ${ }^{31}$ This discovery soon sparked the development of technologies that now allow researchers to study large numbers of cell simultaneously. Although an overview of all types of single cell sequencing technologies goes beyond the scope of this review, there are certain landmarks that must be mentioned, as they also illustrate that the choice of single cell technology is often depending on the experimental question.

In many experimental setups, a primary selection of the cells of interest from an organ or organism is necessary, and one of the most common tools to select cells of interest is Fluorescence Assisted Cell Sorting (FACS). This technology allows for single cells to be identified in a cell suspension and subsequent sorting of the cells in wells of a 96 or 384 well plate that contains lysis buffer and RNAse inhibitors. After this primary selection of cells, the chemistry by which a sequencing library is generated from the mRNA of a single cell can be chosen freely, with the Smart-Seq 2 chemistry commonly used as being the most sensitive and accurate. ${ }^{32}$ Smart-seq 2 allows for the recovery of sequencing information of the entire mRNA molecule, only limited by the efficiency of the reverse transcriptase used to create cDNA. ${ }^{33,34}$ However, Smart-Seq2 is sensitive to PCR-induced biased amplification noise. A solution to this bias is the inclusion of Unique Molecular Identifiers (UMI's), a barcode that is unique to every cDNA molecule in the sequencing library. This allows for accurate counting of mRNA molecules expressed per cell, provided that each mRNA molecule is captured for reverse transcription only once. ${ }^{35}$

The selection of cells using FACS also provides drawbacks. The procedure of cell sorting by itself is already introducing bias, since large and/or delicate cells will not survive the fluidic shear stress induced by the FACS. But most importantly, selection of cells using FACS is limited in its throughput, as most FACS machines are only accurate enough to sort reliably in a 384 well format, thus limiting the liquid handling of the library preparation to a format in which only 384 cells can be analyzed. This makes larger scale single cell analysis using microwell plate based chemistry too inefficient to consider for experimental questions where a large heterogeneity is expected and thousands of cells need to be analyzed. For these experiments, technologies based on droplet encapsulation ${ }^{36}$, capture of cells in microwells ${ }^{37}$ and in situ barcoding ${ }^{38,39}$ are the most prominent ones used today, with the drop-seq implementation commercialized by $10 x$ Genomics being the most popular technology due to its ease of use and simple implementation in research environments. This technology allows the analysis of thousands of cells per sample at a decent gene recovery per cell. Finally, in situ barcoding allows for the analysis of millions of cells simultaneously, however at a comparably low gene recovery per cell. ${ }^{40}$ For very small sample sizes, where 130 
every cell needs to be analyzed in the highest detail, the depth of Smart-Seq2 is preferred, while for samples with enormous complexity (like whole organisms), the width of in situ barcoding or Drop-Seq is needed. This allows researchers, depending on the presence of cell populations in certain organs and pre-enriching techniques like FACS, to decide on which technique is most capable of answering a specific research question. A complete overview of the workflow, from tissue towards bioinformatical analysis, is depicted in Figure 2.

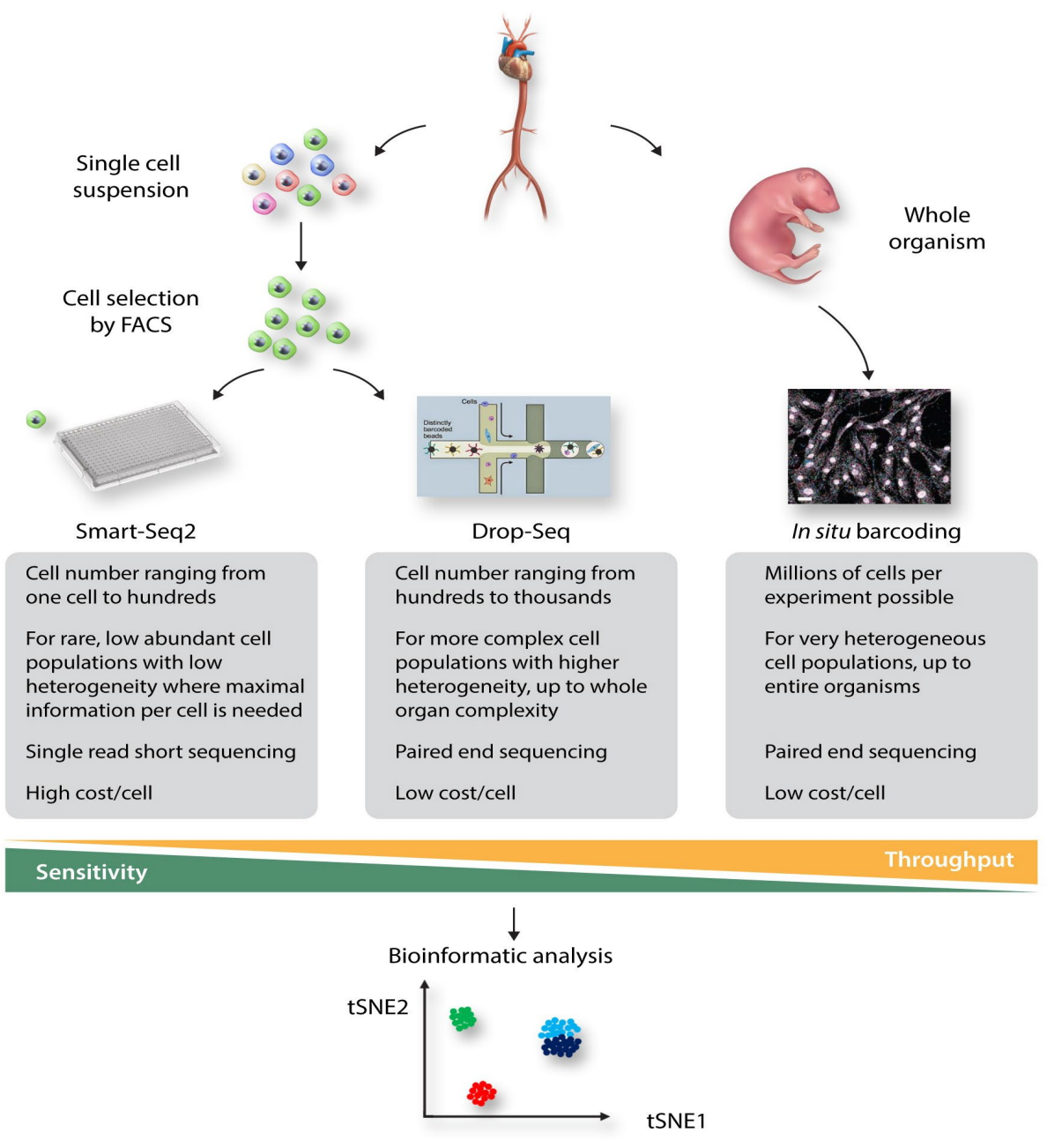

Figure 2. Complete overview from tissue collection, processing, selection, sequencing method and analysis. The advantages and disadvantages from all three sequencing methods are shown in a small diagram within figure 2 . 


\section{4 | Heterogeneity and plasticity in atherosclerosis}

Today, the generation of single cell data is widely accessible to researchers thanks to the plethora of available technologies and their various commercial implementations. However, the proper analysis of single cell data is often not trivial due to the high complexity of the data that it provides. In a dataset, every cell is in essence a separate sample with quantitative information for every single gene, making the data several orders of magnitude richer compared to bulk transcriptomics. Most commonly, the data is visualized using a tstochastic neighbor embedding algorithm (t-SNE). ${ }^{41}$ This algorithm takes the highdimensional data points (i.e. the cells with gene expression information) and reduces this complexity to two dimensions (an X-Y graph). Data points (cells) with high similarity are placed in neighboring positions, with different neighborhoods (often called "clouds" or "data clusters") represented. However, one needs to be aware that t-SNE is a visualization foremost, and that it can easily be tuned to change the look of the data by changing the algorithm's parameters. Also, it is important to remember that the distance between data clusters is not always a measure for difference between cell types, a common misconception. ${ }^{42}$ For this reason, many new algorithms are being developed. Recently, the Uniform Manifold Approximation and Projection (UMAP) algorithm was created, which is similar in its visualization style to t-SNE, but represents the relationship between cell types with higher fidelity. ${ }^{43}$ Another hurdle in single cell data analysis is that the data is often a snapshot in time, while cells in a heterogenous tissue are seldomly static. For example, in a diseased state like atherosclerosis, the SMC are very plastic and to explore the dynamics of the cells, clustering of the cells while preserving the relationship between cell types is paramount. The RNA velocity algorithm allows prediction of future cell states by taking into account the ratio of unspliced versus spliced RNA, which is a measurement of the "age" of the RNA and the activity of the gene that produced it. ${ }^{44}$ Finally, the vasculature is difficult to classify into cell types since the endothelial cells are zonated (i.e. their transcriptome gradually changes according to an anatomical axis. ${ }^{45,46}$ This gradual change in phenotype is well visualized with the Sorting Points Into Neighborhoods (SPIN) algorithm, which sorts all cells on an X-axis according to similarity, while the $\mathrm{Y}$-axis represents the expression level of a chosen zonated gene. ${ }^{47}$ A clustering variant of the SPIN algorithm, BackSPIN, can then be used to split the sorted cells into clusters, if desired. ${ }^{48}$ For a recent overview and discussion on clustering algorithms for single cell data, we would like to refer the reader to an excellent recent review by Kiselev and colleagues. ${ }^{49}$

\section{Healthy vasculature}

All organs have a specific vasculature dedicated to their relative function and this vascular organotypicity has long been recognized. Indeed, the vasculature can present itself as a strong barrier, a highly permeable fenestrated structure, or, as exemplified by the lungs, an interface for facilitated gas exchange. For an excellent overview of organotypic vasculature, we refer the reader to a recent review of Augustin and Koh. ${ }^{50}$ However, the heterogeneity 
of a vascular network within an organ has been studied poorly, until the use of SCS to molecularly define cell types in a vascular network. Recent SCS work has shown that brain and lung endothelial cells are zonated, a term indicating that the transcriptome of cells gradually changes according to an anatomical axis. This thus relates to heterogeneity of cells, as phenotype adaptation does not result in full change or loss of endothelial cell identity. ${ }^{45,46}$ This term was first coined by Jungermann et al, when describing metabolic zonation of the liver hepatocytes, ${ }^{51}$ which later has been extended towards molecular zonation by Halpern et al. using SCS. ${ }^{52}$ The zonation of brain endothelial cells as shown by gradual changes in their transcriptome, related to their position in the vasculature, is schematically shown in Figure 3. 

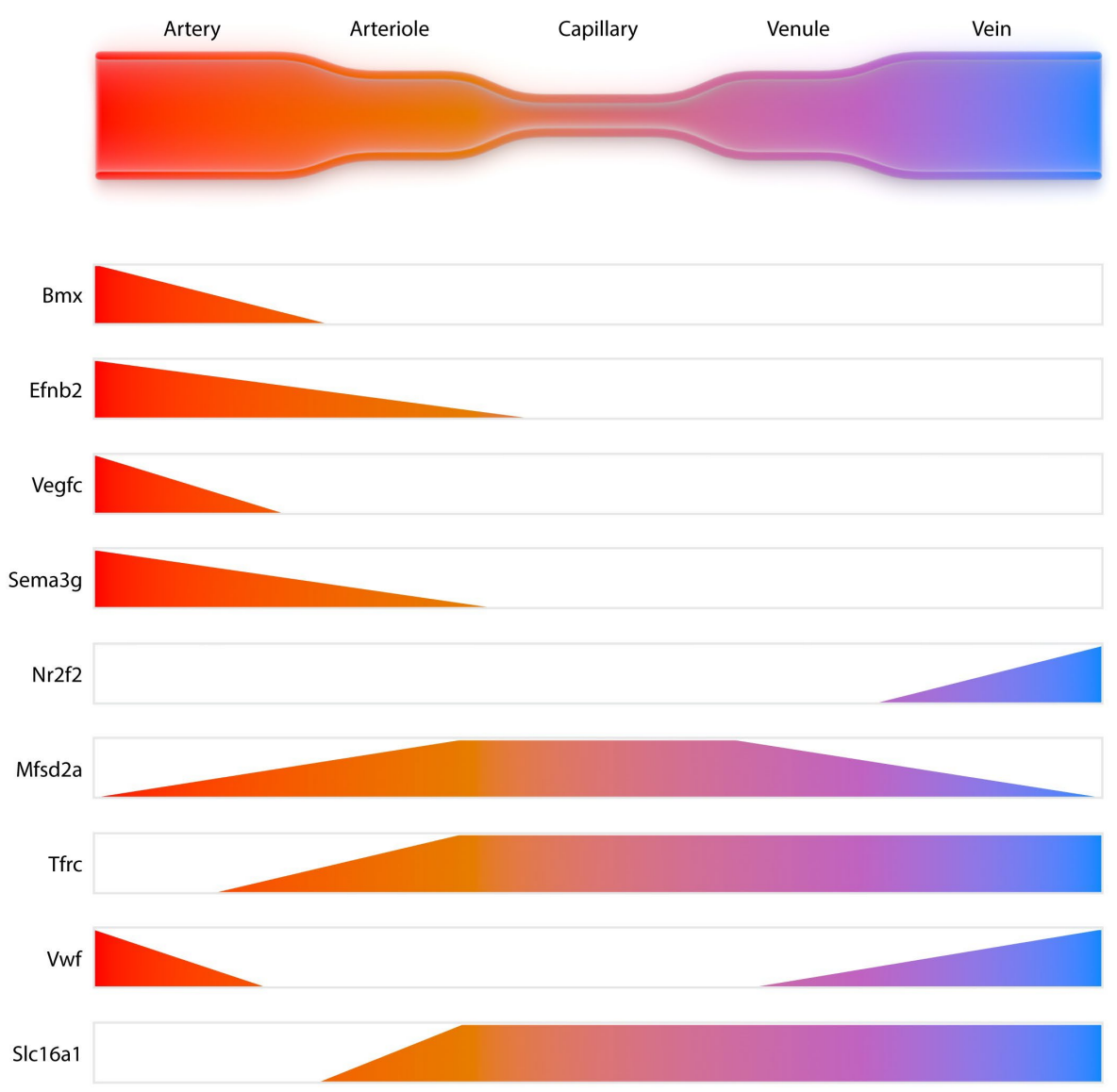

Figure 3. Zonation of endothelial cells in the brain. Gene expression profiles differ along the anatomical axis of the vasculature and thus influence the functional profile of the endothelial cells.

In contrast to the endothelial cells, mural cells (SMC and pericytes) do not present a clear zonated pattern in the brain. While pericytes appear to be largely uniform in their transcriptome and mostly lack expression of genes required for contractility, SMC differ between arteries and veins. Indeed, SMCs on arteries stand out by the strong presence of gene programs required for contractility, while SMCs on veins are more similar to pericytes. Similar zonated patterns were also found in lung vasculature, although strong organotypicity of endothelial cells and pericytes was found. ${ }^{46}$ Furthermore, Vanlandewijck et al. also described the presence of 'fibroblast-like' cells that sit outside of the smooth muscle cell layer, but under the astrocyte end-feet, of the larger arteries and veins. These cells have previously also been referred to as Vascular Leptomeningeal Cells (VLMC's)..$^{53}$ Dobnikar et al provided further support and detail of SMCs heterogeneity using SCS on 
healthy mouse vessels, and was able to show that a small portion of SMCs are positive for stem cell marker Sca-1. ${ }^{54}$ They also showed that this specific subset of SMCs is upregulated during disease. Even more recently, Kalluri et al. and Gu et al. were able to describe vascular heterogeneity in both the healthy murine aorta and adventitia using an unbiased methods starting from all vascular cells. ${ }^{55,56}$ Kalluri isolated total aortic medial and intimal cells without FACS preselection, compared two different enzymatic digestion mixes, sequenced over 6000 cells with low and high sequencing depth (17,000 and 145,000 reads/cell respectively) to define a complete aortic atlas. ${ }^{55}$ Eleven major cell populations were identified with both read depths and enzyme mixes, including SMCs, fibroblasts, monocytes and endothelial cells (EC). They emphasized three phenotypically distinct endothelial cell subsets, revealing different functional aspects. ${ }^{55}$ The major EC subset was enriched for canonical EC markers, however the other two would have been missed by pre-sorting for canonical markers. Differential genes for the second largest subset were involved in angiogenesis, lipid handling, and it was enriched in a tip cell gene signature. The third subset were marked as lymphatic ECs, which together with the large number of fibroblasts, raises the question if the dissection of adventitia from aorta was sufficient, as both cells are mainly thought to reside in the adventitia. However as in total $33 \%$ of all cells appear to be fibroblasts this contradicts the possibility of a small contamination. Validation of tissue RNA or protein localization of these cell types and subsets would add greatly to the biological insight, which is where most current reports are still lacking. However, this report adds considerable insight into the healthy murine cell atlas and shows that cell number is more important for discovery of new subsets than sequencing depth.

The second recent report by Gu et al. made use of SCS to sketch an atlas of all cell types in the adventitia from $\sim 2000$ total cells from healthy and $\sim 3000$ cells from atherosclerotic mice. Gu et al. was able to obtain 15 different cell clusters, including T-cells, B-cells, natural killer cells, monocytes, macrophages and two clusters which they classify as non-immune cells. Despite the relatively small number of non-immune cells ( 800$)$, they showed four different mesenchymal clusters, all linked to specific markers and functional aspects, specifically one cluster was linked to immune cells activation ${ }^{56}$.

Recently, the liver vasculature was investigated with SCS by Halpern et al. using paired-cell sequencing, a method where endothelial/hepatocyte cell pairs are deliberately selected. ${ }^{45}$ Thus, spatial information can be obtained from the zonated profile of the hepatocyte ${ }^{52}$ and endothelial specific gene signatures can be found by subtracting the hepatocyte transcriptome. Although the work has provided interesting insights in liver vascular heterogeneity, the authors also recognize that the dependence on specific paired cells (hepatocytes) is limiting in capturing the complete complexity of the liver vasculature. Further profiling of single cells of the entire vasculature of the liver is warranted.

In order to unify the efforts to create organism-wide single cell atlases, international consortia are formed. Most prominently are the Tabula Muris Consortium and the Human 


\section{4 | Heterogeneity and plasticity in atherosclerosis}

Cell Atlas Consortium, aiming to profile all cell types of the mouse and the human, respectively. In large, organ-wide single cell datasets, the vasculature is also represented, but often the lack of specific focus impedes a molecular characterization of the vasculature for several reasons. First, the separation of endothelial cells from pericytes often requires special dissociation protocols, since they are embedded within the same basement membrane. ${ }^{57}$ As most organ-wide single cell atlas projects are not specifically aiming for the dissociation of these two cell types, an artificial endothelial/pericyte hybrid is commonly described as a cell type of its own. ${ }^{37,58}$ Secondly, the vasculature is often underrepresented or insufficiently subclustered into separate cells, leading to annotation of all vascular cells as "endothelium". ${ }^{59,60}$ For these reasons, specific SCS profiling of vascular beds of healthy, adult organs and the body's main large arteries and veins (aorta, carotid artery, vena cava) is still paramount in establishing a molecular definition of vascular cell types across organs.

\section{Diseased vasculature}

While heterogeneity and zonation are already evident from recent SCS studies using healthy vasculature, heterogeneity is greatly amplified when looking at different disease models. Two reports emerging at the same time focused on immune cell heterogeneity in atherosclerosis. ${ }^{61,62}$ Cochain et al. dived into the immune aspect of atherosclerosis, using mice on a LDL receptor knock-out (LDLR-/-) background for SCS. ${ }^{61} \mathrm{CD} 45$ positive cells from healthy and atherosclerotic tissue were used to investigate immune cell heterogeneity. In total, 13 clusters were found with distinct gene expression patterns, of which 3 clusters were only present in atherosclerotic tissue. These findings clearly show again the cellular adaptability within disease progression, emphasizing the importance of cellular heterogeneity and plasticity in the vasculature. SCS enabled them to find a new gene, triggering receptor expressed on myeloid cells 2 (TREM2), to be highly expressed on a subset of atherosclerotic macrophages, which had not been described before. This subset is involved in lipid metabolism, regulation of cholesterol efflux and oxidative stress, and was previously linked to osteoclasts and disease-associated microglia. Winkels et al. showed a diversity of 11 different clusters of leukocytes based on unsupervised clustering and validate these clusters by using a secondary technique, mass spectrometry cytometry of time of flight (CyTOF). ${ }^{62}$ Even though these papers are leading in the field of immune cell heterogeneity in context of diseased vasculature, there are still some limitations to these studies. Both papers only make use of CD45 positive cells, eliminating the option to look at their communication with other cells within the same tissue. Furthermore, the amount of cells used for analysis could be greatly enhanced.

Aforementioned papers were the first to use SCS as a new technique to investigate immune cell heterogeneity, however, heterogeneity and plasticity of other cells in diseased vasculature have already been described by others over the last years. Hao et al. already proposed SMC heterogeneity in vascular disease back in 2003. ${ }^{63}$ With regards to arterial calcification, location seems to be key in the genetic and functional properties of the 136 
different SMCs. ${ }^{64}$ In atherosclerosis, Chappell et al show that a small subset of very plastic SMCS proliferate extensively, which results in accumulation of SMCs that can gain macrophage markers, like CD107b (MAC3). The given is not only true for atherosclerotic SMCs, but also for those involved in vascular injury. ${ }^{65}$

In recent years, mesenchymal progenitor cells, a plastic and thus heterogeneous cell type by nature, have been getting more and more attention in vascular disease. These cells are thought to originate from the adventitia which is a progenitor niche, according to Majesky et al. ${ }^{26}$ This is supported by earlier data by $\mathrm{Hu}$ et al. who showed clusters of cells in the adventitia of aortic roots, positive for stem cell markers like Sca-1, CD34 and c-Kit. ${ }^{27}$ In addition to the detection of these cells, they also demonstrate their ability to differentiate into SMCs upon PDGF-BB stimulation. Further support for the relevance of arterial progenitor cells, stems from a report showing that VEGF stimulation of CD34+ isolated cells in vitro pushes them to an endothelial cell type with the ability to form small capillaries. ${ }^{66}$ These progenitor cells are not only important in maintaining normal vessel composition, but also play a crucial role in vascular disease. The earlier mentioned study by Hu et al. showed that the transformation of Sca-1+ cells to SMCs is also happening in a murine vein graft in vivo. ${ }^{27}$ When combined with the hyperlipidemic, apolipoprotein E knockout (ApoE-/-) atherosclerosis mouse model, they observed that $20 \%$ of SMCs were Sca-1+ and thus of progenitor origin. These findings are supported by multiple groups who also described the transition of adventitial progenitor cells to SMCs and endothelial cells in the neointima, depending on the stimulus. ${ }^{67-69}$ Furthermore, these mesenchymal cells can generate myofibroblasts and therefore play a role in organ fibrosis, which is not only restricted to the vasculature of large arteries, but also in kidneys, lungs or liver. ${ }^{25}$ The aforementioned SCS study by Gu et al mapping cells in the adventitia of healthy and hypercholesterolemic ApoE/- mice now confirms heterogeneity of adventitial mesenchyme. ${ }^{56}$ However, the relative contribution and function of the observed four mesenchymal clusters was not adapting drastically to the diseased situation. Possibly, the low number of cells ( $\sim 800$ ) prevented full assessment of changes. Interestingly, cross-communication of an inflammatory mesenchymal subset was observed with activated macrophages in the diseased setting. The unbiased approach use to map all cells allowed this important new biological insight ${ }^{56}$. Likewise, the whole aortic medial and intimal cell atlas resulting from the study by Kalluri et al. was derived from an unbiased approach. Here, the observed three EC subsets were conserved upon a high cholesterol diet, while induction of genes involved in collagen turnover suggested the presence of EndMt. Further the relative presence of the main subset was enhanced in diseased, while the opposite was true for the lipid/angiogenic EC subset. This seems rather contradictory to the current knowledge of angiogenic induction upon true hypercholesterolemic disease-settings in double deficient ApoE-/- - LDLr-/- mice. ${ }^{70}$ However the current study involved diet fed wildtype C57BI6 mice representing possibly very early EC dysfunction, not an atherosclerosis model with overt hypercholesterolemia and plaque 


\section{4 | Heterogeneity and plasticity in atherosclerosis}

development. It is therefore very interesting to compare the EC subsets in the - yet unavailable- total cell atlas of atherosclerotic plaques.

All these data together already stress the importance of heterogeneity and/or plasticity of ECs, SMCs, mesenchymal cells and immune cells in the vasculature and how this can affect vascular disease progression. Published SCS data on large, diseased arteries is currently limited to murine studies, only on atherosclerosis, with no data yet available on endothelial cells in atherosclerotic models or on other large artery pathologies, such as pulmonary hypertension, and aneurysms. However, further support for disease-driven amplification of heterogeneity can be gained from a non-cardiovascular model, i.e. hyperpermeable tumor microvasculature. Zhao et al. used human xenografts implanted in mice and detected tumor heterogeneity in endothelial and mesenchymal cells, linked to Notch signaling. ${ }^{71}$ Also Lambrechts et al. made use of SCS in a lung cancer model in mice, separating stromal cells into 52 different subsets with their own gene signature. ${ }^{72}$ Bian et al. even combined single cell transcriptome data with methylome and mutation data of human colorectal cancer samples, broadening the genetic fingerprint all the more so. ${ }^{73}$ Data from tumor microvasculature again confirms the heterogeneity in the vasculature and how single cell sequencing gave more insight in processes involved, e.g. the methylome.

To summarize, these data show the complexity of murine vasculature and how SCS enables us look at different cell types and their gene expression patterns on a deeper level than ever before. This could impact the identification of cell types and new subtypes, since SCS gives more depth to expression patterns belonging to different cell types and their subpopulations. However, few studies go beyond description of the subsets, and it is yet to be resolved if there are actual implications for functional heterogeneity. Further investigation of functional heterogeneity and cell-cell interactions in human atherosclerotic tissue can elucidate processes involved in disease and how the compares between physiology and pathology.

\section{Biological implications}

Thus far, the first groups confirmed the basal atherosclerotic plaque immune cell compositions and have described subtype heterogeneity thereof, and uncovered a potential new macrophage subtype, while SCS of ECs in healthy brain and lung vasculature revealed EC zonation, and arterial- and venous-specific SMC types. ${ }^{45,46,61,62}$ Broadly, we see biological implications related to cell type identity and the pathogenesis of disease.

The introduction of SCS has challenged the classical definition of cell types, which was determined by morphology, tissue location and a few cell identity markers. Bulk transcriptomics and fluorescent reporter mice have already changed this simplified view, and uncovered new subtypes and trans differentiated cells. This distinction between heterogeneity and/or plasticity of cells is often ambiguous and open to errors. In the past, these errors have been made due to lack of high resolution microscopy in 3 dimensions, the lack of specific cell identity markers used for CRE reporters, and the analysis of population 138 
averages, obscuring individual differences and subpopulations. SCS has the potential to clearly distinguish between heterogeneity and plasticity. Grouping cells with similar transcriptomes will identify complete gene signatures of cell identity, validating classical identity markers and uncovering new ones. Clustering tools will enable dissecting major cell types with very distinct cell identity marker signatures, from subtypes whose signatures differ within the boundaries of a cell type signature. Although SCS may simplify the distinction, ambiguity may still exist when there is no real end-stage identity, such as in a dynamic and reversible process like EndMT. Hopefully, detailed pseudotime bioinformatics analysis of the temporal changes in the transcriptome in a controlled experimental setting, may further resolve these issues.

Upon consensus of cell type and subtype identity signatures, and the functional implications thereof, there may arise opportunities for improved resolution of disease. While general anti-inflammation therapy in humans has shown proof-of-concept, it only prevented the relative risk of clinical events by $15 \%{ }^{2}$ Speculating about the potential future advances this insight from SCS could bring the field, raises the possibility of new, subtype selective imaging targets and/or adaptable regulation of cell and subtypes. Adapting therapy to selectively inhibit immune cell subtypes with a detrimental function, or to trigger the conversion into a cell subtype with a more beneficial function could in theory be more effective to prevent clinical events. In future, the adaptable regulation of cell types and subtypes, potentially even in a personalized manner, is expected to have a durable effect on improving life expectancy, quality of life, and avoiding unnecessary treatments. Nevertheless, development and delivery of such subtype-specific inhibitors or reprogramming agents are far more clear and many hurdles need to be taken.

\section{Future technical improvements}

Although clearly important new insights are gained from latest SCS reports, several improvements can be made, both on the technological level, as well as bioinformatics. Here we will discuss limitations and solutions to incomplete genome coverage, number of replicates and how to deal with stoichiometry, low throughput, loss of spatial information and cellular interaction, the need for fresh material, as well as highlight new technologies and analysis tools.

One major limitation of the current technologies is that not the entire transcriptome of individual cells can be mapped and thus, every single cell transcriptome is but a stochastic sample of the pool of mRNA present in that cell In addition, only highly expressed noncoding RNAs can now be identified. However, the sensitivity of the methods is continuously improving allowing the detection of more and more genes in every individual cell, as well as non-coding RNAs. Recently, an optimized version of SCRB-Seq was developed called mcSCRB-Seq ${ }^{74}$ (molecular crowding single-cell RNA Barcoding and sequencing) using the molecular crowding agent PEG (polyethylenglycol), which increases the efficiency of the RT 


\section{4 | Heterogeneity and plasticity in atherosclerosis}

(reverse transcription) reaction in a concentration dependent manner. Thus, this protocol is at the moment the most sensitive plate-based single cell RNA-Seq protocol (benchmarked using ERCCS). In addition, 10X genomics recently released a new version of the $3^{\prime}$ single RNA-Seq assay with a higher capture capacity of polyadenlylated RNA, thus leading to more detected genes per cell.

The matter of biological replicates is also important to consider. Many published studies use a single sample, or a single pool of samples for the assessment of heterogeneity in an organ or disease condition. Although a single sample is already very informative in exploring cellular heterogeneity, it is often dangerous to extrapolate the result into a definite 'atlas'. Taking into account current pricing of SCS, the trade-off between using more replicates, comparing different models or including more time-points is often hard to decide upon, and is very specific to the experimental question. ${ }^{75,76}$

It is obvious that more replicates would increase the robustness of the data, yet one has to take batch effects into account that occur with every RNA-Seq reaction. To minimize batch effects, different experimental groups should ideally not be on separate days. With the use of barcoded oligos, different samples can be pooled in one reaction for droplet based assays, like $10 \mathrm{X}$ genomics, thus reducing batch effects. Sometimes, this may not be applicable due to low number of cells, but bioinformatic tools exist that allow the correction for batch effects. ${ }^{77}$

High-throughput analysis of multiple "omics" on single cell level will likely provide new biological insights into tissue heterogeneity and disease development. Single-cell RNA sequencing has evolved to a high-throughput technology with the development of technological advances like combinatorial indexing or droplet based technologies, which reduced costs and increased throughput to over 100.000 cells that can be analyzed in one experiment. ${ }^{38,39,78,79}$ Indeed, early studies may have been hindered by low cell numbers studied, obscuring the identification of rare (sub)populations. Higher throughput of cells would allow inclusion of all cells in an organ, and with appropriate bioinformatics their interaction could then also be mapped. This could for instance be achieved by studying receptor-ligand interaction pairs as described by Skelly et al. in the mouse heart. ${ }^{80}$ In addition to cell-cell interactions, also spatial information can now be retrieved. Most singlecell analysis experiments start with the dissociation of single cells from tissues, so that spatial information is lost. One solution to regain spatial information has been demonstrated by Halpern et. al. who used a panel of zonated landmark genes with smFISH to remap the single cell transcriptomes of mouse liver cells to the zonation profile..$^{52}$ Other approaches are osmFISH or huluFISH. ${ }^{81,82}$ Techniques for direct in-situ transcriptomics have also been described (e.g. MERFISH, STARmap). ${ }^{83,84}$

The majority of studies report on murine material as proof of principle, due to need for fresh, homogeneous samples, necessitating fewer biological replicates. To speed up human discovery, the use of frozen, bio-archived material would make large sets of previously collected, frozen patient material available. The current use of fresh material for droplet- 
based technology, hinders the step to large scale collection of human samples, usually presenting one by one. In addition to larger heterogeneity compared to animal models, this adds potential batch effects and might obscure disease-related transcriptional changes. Although some reports claim that transcriptomics are comparable between fresh and frozen samples ${ }^{85}$, recently also isolation protocols and studies of single nuclei have been evolved that allow the analysis of bio-archived tissues. ${ }^{86}$ These isolation protocols also reduce the isolation bias that comes with tissue dissociation protocols resulting in better isolation of some cell types compared to others. Furthermore nuclear isolation might minimize transcriptional changes during the isolation process since the full isolation can be carried out at $4^{\circ} \mathrm{C}$, as no enzymatic digestion is needed. ${ }^{87}$

The aforementioned technological advancements can overcome some of the current limitations. We will briefly highlight other developments allowing, i.e. simultaneous quantifications of protein levels, multiplexing of samples, and sequencing of the active transcriptome. The addition of oligonucleotide based barcoded antibodies to the single cell suspension has added protein expression abundance on the cell surfaces to the sequencing data called Cite-Seq. ${ }^{88}$ This technique was also developed to combine more sample in one reaction e.g. on the $10 X$ Chromium to reduce batch effects and study more cells called cellhashing. Another approach to multiplex several samples from different individuals in one single cell experiment by using genetic variation of individuals has been recently described. ${ }^{89}$ Further developments to study intracellular proteins or phosphoproteins are being developed..$^{90}$ Another exciting technology is the mapping of open chromatin regions in single cells. ${ }^{91}$ Using combinatorial indexing techniques or commercially kits, single ATAC sequencing has now become available for high throughput analysis. ${ }^{92}$ The additional DNA accessibility information in detected cell populations combined with mRNA-expression data from regular scRNA-seq will certainly help to identify novel cell populations and also validate the mRNA expression data on whether a detected population is truly distinct from the other cell-populations. An additional level has been recently added to this using FANS (fluorescent associated nuclei sorting) and single nuclear (sn)ATAC. ${ }^{92,93}$ snATAC allows the discovery of unique enhancer regions and regulatory logic in distinct cell types but, due to the nature of the data, does not allow the same accuracy of unsupervised clustering as with scRNA-Seq data. Analysis of both datasets scRNA-seq and scATAC-seq complements each other and allows among other things the identification of rare cell clusters. ${ }^{94}$

In addition, bioinformatics tools are also evolving to accommodate current limitations. Much can be gained from in-depth bioinformatics such as pseudo-time trajectory analysis to study cellular trans-differentiation in detail. New exciting computational tools that allow pseudo-time analysis in single cell data have been developed and refined. ${ }^{95,96}$ As further techniques are being developed, bioinformatical integration of multi-omics datasets of single cell analysis represents a major challenge. ${ }^{97}$ 


\section{4 | Heterogeneity and plasticity in atherosclerosis}

\section{Conclusions}

This review emphasizes the importance of cell heterogeneity and plasticity in healthy vasculature and how this relates to atherosclerosis development and progression. We discussed single cell sequencing (SCS) as a very useful technique in further investigating cell heterogeneity and plasticity. SCS has given the opportunity to link gene expression patterns to classical cell types and their subpopulations, but also how these patterns vary upon different environmental stimuli, challenging the plasticity of these cells. The depth in which SCS can offer genetic insight is dependent on the method chosen by researchers. Where Smart-Seq2 offers researchers the possibility to investigate expression of full-length RNA in cells, it limits the number processed cells per batch to 384. On the other hand, Drop-seq and in situ barcoding enable researchers to use larger quantities of cells or even complete embryos, but with lower gene recovery per cell. Development of new bioinformatic analysis tools is emerging and is allowing researchers analyze more information, such as RNA splicing or zonation. The latter seems of great importance regarding cell heterogeneity, which is proven in multiple organs in healthy state like liver, lung and the brain as shown for the latter by Vanlandewijck et al. ${ }^{46}$ Heterogeneity and plasticity of endothelial cells, SMCs, immune cells and mesenchymal cells has shown to be present in healthy vasculature, but is even more amplified in diseased vasculature. Current studies highlight this by using SCS in studying changes in cell populations and gene expression patterns in atherosclerotic mouse models. However, these studies are still only limited to murine models, since only fresh material can be used. Nowadays more advanced methods, such as Single Nucleus RNAseq, are broadening the field with the use of frozen tissue and thus also adding possibility of using human biopsies from tissue banks, expanding single cell knowledge across species. This insight could help to identify novel therapeutic targets and pave the way towards urgently needed novel targeted therapeutics for the vast and growing patient population suffering from cardiovascular disease. However, latest and future advancements in technology and bio-informatics should be implemented to drive the insight from SCS data from mere description of existing and new subpopulations towards a full, in-depth insight into functional and spatial heterogeneity in vivo and cell-cell communication in healthy and diseased vasculature. 


\section{Funding}

This work has been supported by the Netherlands Scientific Organization [NWO Vidi 91718364 to J.C.S], the German Society of Internal Medicine (DGIM) as part of the clinician scientist program [C.K.], German Research Foundation [SFB TRR219 to R.K.], The Swedish Science Council [2015-00550 to M.V. and C.B.], the Swedish Cancer Society [150735 to M.V. and C.B.], the Knut and Alice Wallenberg Foundation [2015:0030 to M.V. and C.B.] and the Leducq Foundation [14CVD02 to M.V. and C.B.].

\section{Conflict of interest}

The authors declare no conflict of interest. 


\section{4 | Heterogeneity and plasticity in atherosclerosis}

\section{References}

1. Herrington W, Lacey B, Sherliker P, et al. Epidemiology of Atherosclerosis and the Potential to Reduce the Global Burden of Atherothrombotic Disease. Circ Res. 2016;118:535-46.

2. Ridker PM, Everett BM, Thuren T, et al. Antiinflammatory Therapy with Canakinumab for Atherosclerotic Disease. N Engl J Med. 2017;377:1119-1131.

3. Ridker PM, MacFadyen JG, Everett BM, et al. Relationship of C-reactive protein reduction to cardiovascular event reduction following treatment with canakinumab: a secondary analysis from the CANTOS randomised controlled trial. Lancet. 2018;391:319328.

4. Nicholls SJ, Puri R, Anderson T, et al. Effect of Evolocumab on Progression of Coronary Disease in Statin-Treated Patients: The GLAGOV Randomized Clinical Trial. JAMA. 2016;316:2373-2384.

5. Tang DG. Understanding cancer stem cell heterogeneity and plasticity. Cell Res. 2012;22:457-72.

6. Loeffler M and Roeder I. Tissue stem cells: definition, plasticity, heterogeneity, selforganization and models--a conceptual approach. Cells Tissues Organs. 2002;171:8-26.

7. Insull W, Jr. The pathology of atherosclerosis: plaque development and plaque responses to medical treatment. Am J Med. 2009;122:S3-S14.

8. Weber $\mathrm{C}$ and Noels $\mathrm{H}$. Atherosclerosis: current pathogenesis and therapeutic options. Nat Med. 2011;17:1410-22.

9. Linton MF, Babaev VR, Huang J, et al. Macrophage Apoptosis and Efferocytosis in the Pathogenesis of Atherosclerosis. Circ J. 2016;80:2259-2268.

10. Gonzalez L and Trigatti BL. Macrophage Apoptosis and Necrotic Core Development in Atherosclerosis: A Rapidly Advancing Field with Clinical Relevance to Imaging and Therapy. Can J Cardiol. 2017;33:303-312.

11. Martinet W, Schrijvers DM and De Meyer GR. Necrotic cell death in atherosclerosis. Basic Res Cardiol. 2011;106:749-60.

12. Aird WC. Endothelial cell heterogeneity and atherosclerosis. Curr Atheroscler Rep. 2006;8:69-75.

13. Doran AC, Meller $\mathrm{N}$ and McNamara CA. Role of smooth muscle cells in the initiation and early progression of atherosclerosis. Arterioscler Thromb Vasc Biol. 2008;28:812-9.

14. Feil S, Fehrenbacher B, Lukowski R, et al. Transdifferentiation of vascular smooth muscle cells to macrophage-like cells during atherogenesis. Circ Res. 2014;115:662-7.

15. Rong JX, Shapiro M, Trogan E, et al. Transdifferentiation of mouse aortic smooth muscle cells to a macrophage-like state after cholesterol loading. Proc Natl Acad Sci U S A. 2003;100:13531-6.

16. Shankman LS, Gomez D, Cherepanova OA, et al. KLF4-dependent phenotypic modulation of smooth muscle cells has a key role in atherosclerotic plaque pathogenesis. Nat Med. 2015;21:628-37.

144 
17. Allahverdian S, Chaabane C, Boukais K, et al. Smooth muscle cell fate and plasticity in atherosclerosis. Cardiovasc Res. 2018;114:540-550.

18. Bennett MR, Sinha S and Owens GK. Vascular Smooth Muscle Cells in Atherosclerosis. Circ Res. 2016;118:692-702.

19. Wilson HM. Macrophages heterogeneity in atherosclerosis - implications for therapy. J Cell Mol Med. 2010;14:2055-65.

20. Nagenborg J, Goossens P, Biessen EAL, et al. Heterogeneity of atherosclerotic plaque macrophage origin, phenotype and functions: Implications for treatment. Eur J Pharmacol. 2017;816:14-24.

21. Xue J, Schmidt SV, Sander J, et al. Transcriptome-based network analysis reveals a spectrum model of human macrophage activation. Immunity. 2014;40:274-88.

22. Johnson JL and Newby AC. Macrophage heterogeneity in atherosclerotic plaques. Curr Opin Lipidol. 2009;20:370-8.

23. Tse K, Tse H, Sidney J, et al. T cells in atherosclerosis. Int Immunol. 2013;25:615-22.

24. Tabas I and Lichtman AH. Monocyte-Macrophages and T Cells in Atherosclerosis. Immunity. 2017;47:621-634.

25. El Agha E, Kramann R, Schneider RK, et al. Mesenchymal Stem Cells in Fibrotic Disease. Cell Stem Cell. 2017;21:166-177.

26. Majesky MW, Dong XR, Hoglund V, et al. The adventitia: a progenitor cell niche for the vessel wall. Cells Tissues Organs. 2012;195:73-81.

27. Hu Y, Zhang Z, Torsney E, et al. Abundant progenitor cells in the adventitia contribute to atherosclerosis of vein grafts in ApoE-deficient mice. J Clin Invest. 2004;113:1258-65.

28. Kramann R, Goettsch C, Wongboonsin J, et al. Adventitial MSC-like Cells Are Progenitors of Vascular Smooth Muscle Cells and Drive Vascular Calcification in Chronic Kidney Disease. Cell Stem Cell. 2016;19:628-642.

29. Evrard SM, Lecce L, Michelis KC, et al. Endothelial to mesenchymal transition is common in atherosclerotic lesions and is associated with plaque instability. Nat Commun. 2016;7:11853.

30. Souilhol C, Harmsen MC, Evans PC, et al. Endothelial-mesenchymal transition in atherosclerosis. Cardiovasc Res. 2018;114:565-577.

31. Tang F, Barbacioru C, Wang Y, et al. mRNA-Seq whole-transcriptome analysis of a single cell. Nat Methods. 2009;6:377-82.

32. Ziegenhain C, Vieth B, Parekh S, et al. Comparative Analysis of Single-Cell RNA Sequencing Methods. Mol Cell. 2017;65:631-643 e4.

33. Picelli S, Bjorklund AK, Faridani OR, et al. Smart-seq2 for sensitive full-length transcriptome profiling in single cells. Nat Methods. 2013;10:1096-8.

34. Picelli S, Faridani OR, Bjorklund AK, et al. Full-length RNA-seq from single cells using Smart-seq2. Nat Protoc. 2014;9:171-81. 


\section{4 | Heterogeneity and plasticity in atherosclerosis}

35. Islam S, Zeisel A, Joost $S$, et al. Quantitative single-cell RNA-seq with unique molecular identifiers. Nat Methods. 2014;11:163-6.

36. Macosko EZ, Basu A, Satija R, et al. Highly Parallel Genome-wide Expression Profiling of Individual Cells Using Nanoliter Droplets. Cell. 2015;161:1202-1214.

37. Han X, Wang R, Zhou Y, et al. Mapping the Mouse Cell Atlas by Microwell-Seq. Cell. 2018;172:1091-1107 e17.

38. Cao J, Packer JS, Ramani V, et al. Comprehensive single-cell transcriptional profiling of a multicellular organism. Science. 2017;357:661-667.

39. Rosenberg $A B$, Roco CM, Muscat RA, et al. Single-cell profiling of the developing mouse brain and spinal cord with split-pool barcoding. Science. 2018;360:176-182.

40. Cao J, Spielmann M, Qiu X, et al. The single-cell transcriptional landscape of mammalian organogenesis. Nature. 2019;566:496-502.

41. van der Maaten L HG. Visualizing Data using t-SNE. Journal of Machine Learning Research. 2008;9:2579-2605.

42. Wattenberg M VF, Johnson I. How to use t-SNE effectively. Distill. 2016.

43. Becht E, Mclnnes L, Healy J, et al. Dimensionality reduction for visualizing singlecell data using UMAP. Nat Biotechnol. 2018.

44. La Manno G, Soldatov R, Zeisel A, et al. RNA velocity of single cells. Nature. 2018;560:494-498.

45. Halpern KB, Shenhav R, Massalha $H$, et al. Paired-cell sequencing enables spatial gene expression mapping of liver endothelial cells. Nat Biotechnol. 2018;36:962-970.

46. Vanlandewijck M, He L, Mae MA, et al. A molecular atlas of cell types and zonation in the brain vasculature. Nature. 2018;554:475-480.

47. Tsafrir D, Tsafrir I, Ein-Dor L, et al. Sorting points into neighborhoods (SPIN): data analysis and visualization by ordering distance matrices. Bioinformatics. 2005;21:2301-8.

48. Zeisel A, Munoz-Manchado AB, Codeluppi S, et al. Brain structure. Cell types in the mouse cortex and hippocampus revealed by single-cell RNA-seq. Science. 2015;347:113842.

49. Kiselev VY, Andrews TS and Hemberg M. Challenges in unsupervised clustering of single-cell RNA-seq data. Nat Rev Genet. 2019.

50. Augustin HG and Koh GY. Organotypic vasculature: From descriptive heterogeneity to functional pathophysiology. Science. 2017;357.

51. Jungermann $\mathrm{K}$ and Katz N. Functional hepatocellular heterogeneity. Hepatology. 1982;2:385-95.

52. Halpern KB, Shenhav R, Matcovitch-Natan O, et al. Single-cell spatial reconstruction reveals global division of labour in the mammalian liver. Nature. 2017;542:352-356.

53. Marques S, Zeisel A, Codeluppi S, et al. Oligodendrocyte heterogeneity in the mouse juvenile and adult central nervous system. Science. 2016;352:1326-1329. 
54. Dobnikar L, Taylor AL, Chappell J, et al. Disease-relevant transcriptional signatures identified in individual smooth muscle cells from healthy mouse vessels. Nat Commun. 2018;9:4567.

55. Kalluri AS, Vellarikkal SK, Edelman ER, et al. Single Cell Analysis of the Normal Mouse Aorta Reveals Functionally Distinct Endothelial Cell Populations. Circulation. 2019.

56. Gu W, Ni Z, Tan YQ, et al. Adventitial Cell Atlas of wt (Wild Type) and ApoE (Apolipoprotein E)-Deficient Mice Defined by Single-Cell RNA Sequencing. Arterioscler Thromb Vasc Biol. 2019;39:1055-1071.

57. Armulik A, Genove $G$ and Betsholtz C. Pericytes: developmental, physiological, and pathological perspectives, problems, and promises. Dev Cell. 2011;21:193-215.

58. Zeisel A, Hochgerner H, Lonnerberg P, et al. Molecular Architecture of the Mouse Nervous System. Cell. 2018;174:999-1014 e22.

59. Sohni A, Tan K, Song HW, et al. The Neonatal and Adult Human Testis Defined at the Single-Cell Level. Cell Rep. 2019;26:1501-1517 e4.

60. Tabula Muris Consortium. Single-cell transcriptomics of 20 mouse organs creates a Tabula Muris. Nature. 2018;562:367-372.

61. Cochain C, Vafadarnejad E, Arampatzi P, et al. Single-Cell RNA-Seq Reveals the Transcriptional Landscape and Heterogeneity of Aortic Macrophages in Murine Atherosclerosis. Circ Res. 2018;122:1661-1674.

62. Winkels $H$, Ehinger $E$, Vassallo $M$, et al. Atlas of the Immune Cell Repertoire in Mouse Atherosclerosis Defined by Single-Cell RNA-Sequencing and Mass Cytometry. Circ Res. 2018;122:1675-1688.

63. Hao H, Gabbiani $G$ and Bochaton-Piallat ML. Arterial smooth muscle cell heterogeneity: implications for atherosclerosis and restenosis development. Arterioscler Thromb Vasc Biol. 2003;23:1510-20.

64. Espitia O, Chatelais $\mathrm{M}$, Steenman $\mathrm{M}$, et al. Implication of molecular vascular smooth muscle cell heterogeneity among arterial beds in arterial calcification. PLoS One. 2018;13:e0191976.

65. Chappell J, Harman JL, Narasimhan VM, et al. Extensive Proliferation of a Subset of Differentiated, yet Plastic, Medial Vascular Smooth Muscle Cells Contributes to Neointimal Formation in Mouse Injury and Atherosclerosis Models. Circ Res. 2016;119:1313-1323.

66. Pasquinelli G, Tazzari PL, Vaselli C, et al. Thoracic aortas from multiorgan donors are suitable for obtaining resident angiogenic mesenchymal stromal cells. Stem Cells. 2007;25:1627-34.

67. Chen $\mathrm{Y}$, Wong MM, Campagnolo $\mathrm{P}$, et al. Adventitial stem cells in vein grafts display multilineage potential that contributes to neointimal formation. Arterioscler Thromb Vasc Biol. 2013;33:1844-51. 


\section{4 | Heterogeneity and plasticity in atherosclerosis}

68. Tsai TN, Kirton JP, Campagnolo $P$, et al. Contribution of stem cells to neointimal formation of decellularized vessel grafts in a novel mouse model. Am J Pathol. 2012;181:362-73.

69. Kokkinopoulos I, Wong MM, Potter CMF, et al. Adventitial SCA-1(+) Progenitor Cell Gene Sequencing Reveals the Mechanisms of Cell Migration in Response to Hyperlipidemia. Stem Cell Reports. 2017;9:681-696.

70. Kampschulte M, Stockl C, Langheinrich AC, et al. Western diet in ApoE-LDLR double-deficient mouse model of atherosclerosis leads to hepatic steatosis, fibrosis, and tumorigenesis. Lab Invest. 2014;94:1273-82.

71. Zhao Q, Eichten A, Parveen A, et al. Single-Cell Transcriptome Analyses Reveal Endothelial Cell Heterogeneity in Tumors and Changes following Antiangiogenic Treatment. Cancer Res. 2018;78:2370-2382.

72. Lambrechts D, Wauters E, Boeckx B, et al. Phenotype molding of stromal cells in the lung tumor microenvironment. Nat Med. 2018;24:1277-1289.

73. Bian S, Hou Y, Zhou X, et al. Single-cell multiomics sequencing and analyses of human colorectal cancer. Science. 2018;362:1060-1063.

74. Bagnoli JW, Ziegenhain C, Janjic A, et al. Sensitive and powerful single-cell RNA sequencing using mcSCRB-seq. Nat Commun. 2018;9:2937.

75. Kleyman M SE, Nicola T, Espinoza C, Chhabra D, Hagood J.S., Kaminski N, Ambalavanan N, Bar-Jospeh Z. Selecting the most appropriate time points to profile in highthroughput studies. eLife. 2017.

76. Sefer E KM, Bar-Joseph Z. Tradeoffs between Dense and Replicate Sampling Strategies for High-Throughput Time Series Experiments. Cell Systems. 2016;3:35-42.

77. Haghverdi L, Lun ATL, Morgan MD, et al. Batch effects in single-cell RNA-sequencing data are corrected by matching mutual nearest neighbors. Nat Biotechnol. 2018;36:421427.

78. Wang $\mathrm{Y}$ and Navin NE. Advances and applications of single-cell sequencing technologies. Mol Cell. 2015;58:598-609.

79. Chen X TS, Meyer KB. From tissues to cell types and back: Single-cell gene expression analysis of tissue architecture. Annual Review of Biomedical Data Science. 2018;1:29-51.

80. Skelly DA, Squiers GT, McLellan MA, et al. Single-Cell Transcriptional Profiling Reveals Cellular Diversity and Intercommunication in the Mouse Heart. Cell Rep. 2018;22:600-610.

81. Codeluppi S, Borm LE, Zeisel A, et al. Spatial organization of the somatosensory cortex revealed by osmFISH. Nat Methods. 2018;15:932-935.

82. Cheng YS ZY, Hartmann K, Zou P, Bekki G, Alter H, Liu HK. Autonomous Combinatorial Color Barcoding For Multiplexing Single Molecule RNA Visualization. 2017.

83. Chen $\mathrm{KH}$, Boettiger AN, Moffitt JR, et al. RNA imaging. Spatially resolved, highly multiplexed RNA profiling in single cells. Science. 2015;348:aaa6090. 
84. Wang $X$, Allen WE, Wright MA, et al. Three-dimensional intact-tissue sequencing of single-cell transcriptional states. Science. 2018;361.

85. Wu H, Kirita Y, Donnelly EL, et al. Advantages of Single-Nucleus over Single-Cell RNA Sequencing of Adult Kidney: Rare Cell Types and Novel Cell States Revealed in Fibrosis. Journal of the American Society of Nephrology : JASN. 2019;30:23-32.

86. Habib N, Avraham-Davidi I, Basu A, et al. Massively parallel single-nucleus RNA-seq with DroNc-seq. Nat Methods. 2017;14:955-958.

87. Nawijn M CO, Vieira Brage F, Berg M, Brouwer S, Kar G, Teichmann S, Van den Berge, M. Novel cell types and altered cell states in asthma revealed by single-cell RNA sequencing of airway wall biopsies. European Respiratory Journal. 2018;52.

88. Stoeckius M, Hafemeister C, Stephenson W, et al. Simultaneous epitope and transcriptome measurement in single cells. Nat Methods. 2017;14:865-868.

89. Kang HM, Subramaniam M, Targ S, et al. Multiplexed droplet single-cell RNAsequencing using natural genetic variation. Nat Biotechnol. 2018;36:89-94.

90. Gerlach JP, van Buggenum JAG, Tanis SEJ, et al. Combined quantification of intracellular (phospho-)proteins and transcriptomics from fixed single cells. Sci Rep. 2019;9:1469.

91. Cao J CD, Ramani V, Aghamirzaie D, Pliner H, Hill AJ, Daza RM, McFaline-Figueroa JL, Packer JS, Christiansen L, Steemers FJ, Adey AC, Trapnell C, Shendure J. Joint profiling of chromatin accessibility and gene expression in thousands of single cells. Science. 2018;361:1380-1385.

92. Cusanovich DA, Daza R, Adey A, et al. Multiplex single cell profiling of chromatin accessibility by combinatorial cellular indexing. Science. 2015;348:910-4.

93. Chen X, Miragaia RJ, Natarajan KN, et al. A rapid and robust method for single cell chromatin accessibility profiling. Nat Commun. 2018;9:5345.

94. Stuart T BA, Hoffman P, Hafemeister C, Papalexi E, Mauck III WM, Stoeckius M, Smibert P, Satija R Comprehensive integration of single cell data. BioRxiv. 2018.

95. Qiu X, Hill A, Packer J, et al. Single-cell mRNA quantification and differential analysis with Census. Nat Methods. 2017;14:309-315.

96. Trapnell C, Cacchiarelli D, Grimsby J, et al. The dynamics and regulators of cell fate decisions are revealed by pseudotemporal ordering of single cells. Nat Biotechnol. 2014;32:381-386.

97. Barkas N PV, Nikolaeva D, Lozinsky Y, Demharter S, Khodosevich K, Kharchenko PV. Wiring together large single-cell RNA-seq sample collections BioRxiv 460246. 2018. 



\section{Chapter 5}

\section{Deficiency of myeloid PHD proteins aggravates atherogenesis via macrophage apoptosis and paracrine fibrotic signaling}

van Kuijk K†, Demandt JAF†, Perales-Patón J†, Theelen TL, Kuppe C, Marsch E, de Bruijn J, Jin H, Gijbels MJ, Matic L, Mees BME, Reutelingsperger CPM, Hedin U, Biessen EAL, Carmeliet P, Baker AH, Kramann RK, Schurgers LJ, Saez-Rodriguez J, Sluimer JC

†Authors contributed equally

In revision Cardiovascular Research 



\section{Abstract}

Atherosclerotic plaque hypoxia is detrimental for macrophage function. Prolyl hydroxylases (PHDs) initiate cellular responses to hypoxia, possibly influencing macrophage function in plaque hypoxia. Thus, we aimed to elucidate the role of myeloid PHDs in atherosclerosis. Myeloid specific PHD knock-out (PHDKO) mice were obtained via bone marrow transplantation (PHD1KO, PHD3KO) or conditional knockdown through lysozyme M-driven Cre recombinase (PHD2cKO). Mice were fed high cholesterol diet for 6-12 weeks to induce atherosclerosis. Aortic root plaque size was augmented 2.6-fold in PHD2cKO, and 1.4-fold in PHD3KO, but was unchanged PHD1KO mice. Macrophage apoptosis was promoted in PHD2CKO and PHD3KO mice in vitro and in vivo via the HIF1 $\alpha / B N I P 3$ axis. Bulk and single cell RNA data of PHD2cKO bone-marrow-derived macrophages (BMDM) and plaque macrophages, respectively, confirmed these findings and were validated by siRNA silencing. Human plaque BNIP3 mRNA associated with carotid plaque necrotic core content, suggesting similar effects. Further, PHD2cKO plaques displayed enhanced fibrosis, independent of macrophage function. PHD2cKO BMDMs enhanced fibroblast collagen secretion in a paracrine manner and in silico analysis of macrophage-fibroblast communication predicted SPP1 signaling as regulator, confirmed by enhanced SPP1 protein in vivo. Enhanced SPP1 expression upon PHD2cKO was specifically observed in foamy plaque macrophages expressing "triggering receptor expressed on myeloid cells-2" (TREM2) in our single cell RNA dataset, but not in neutrophils. This confirmed aggravated fibrotic signaling in vitro as well as in vivo.

Myeloid PHD2cKO and PHD3KO enhanced plaque growth, macrophage apoptosis, while PHD2cKO macrophages activated paracrine collagen secretion by fibroblasts in vitro, likely via SPP1. This was in line with enhanced collagen accumulation and SPP1 expression in plaques and by TREM2-foamy plaque macrophages in vivo. 


\section{5 | Myeloid PHD deficiency in atherosclerosis}

\section{Introduction}

Atherosclerosis is driven by macrophages, which comprise the major myeloid subset of the plaque. ${ }^{1,2}$ Macrophages attempt to clear cholesterol and cellular debris accumulated in the intimal wall. However, their function is inhibited as plaque macrophages are hypoxic as a result of their high metabolic demand. ${ }^{3,4}$ Restoring murine plaque oxygenation can decrease necrotic plaque content by improving macrophage function. ${ }^{3}$ This indicates that plaque hypoxia is an active participant, rather than an epiphenomenon in atherogenesis.

Three prolyl hydroxylases, (PHD) 1, 2 and 3, are of fundamental importance in hypoxic signaling. ${ }^{5,6}$ PHDs use oxygen to hydroxylate the transcription factors hypoxia inducible factor (HIF) $1 \alpha$ and $2 \alpha$, marking their degradation. Together, PHDs regulate the activity of HIF1 $\alpha$ and HIF $2 \alpha$, and downstream mechanisms in order to alleviate detrimental hypoxic effects. Each PHD has a different intra-cellular localization and affinity for HIF1 $\alpha$ and HIF2 $\alpha$. HIF1 $\alpha$ and HIF $2 \alpha$ are also involved in macrophage inflammatory pathways, in which they exert both unique and opposing functions. ${ }^{7}$ The effect of HIF2 $\alpha$ deficiency in atherogenesis is not studied, while the role of HIF1 $\alpha$ is controversial. ${ }^{8,9}$ Thus, studying the role of their upstream regulators is important to fully elucidate hypoxic signaling in macrophage function. In addition, PHDs are shown to have HIF independent effects. ${ }^{10}$ Systemic protective effects of whole-body PHD1 and 2 deficiency, and pan-PHD inhibitors on mouse and human cholesterol metabolism and atherogenesis were attributed to stromal cells. ${ }^{11,12}$ However, the detailed mechanistic role for PHDs in plaque macrophage function remains elusive. Thus, we studied the effects of PHD protein signaling in myeloid cells on plaque development and phenotype. 


\section{Methods}

\section{Animals}

All mouse experiments were approved by the regulatory authority of the Maastricht University Medical Centre and performed in compliance with the Dutch governmental guidelines and directive 2010/63/EU of the European Parliament on the protection of animals used for scientific purposes. Whole-body PHD1KO and PHD3KO mice, ${ }^{13,}{ }^{14}$ PHD2 conditional knock-out mice (PHD2cKO) ${ }^{15}$ and LysMCre transgenics, ${ }^{16}$ were previously described. All PHD lines were crossed to low density lipoprotein receptor knock-out (LDLRKO) mice, obtained from an in-house breeding colony, originating from Charles River (Wilmington, MA, USA) and refreshed every 10 generations to avoid genetic drift. Male LDLRKO mice were used as control in all experiments involving PHD1KO and PHD3KO. LysMCre LDLRKO mice (hereafter referred to as PHD2 WT) served as control in all experiments with PHD2cKO mice. An overview of all mouse models is shown in Supplemental (S) Figure S1. All mice were crossed back on LDLRKO C57BI6/J background at least nine times. Animals were housed in the laboratory animal facility of Maastricht University under standard conditions. Food and water were provided ad libitum during the entire experiment. All animals were housed in individually ventilated cages (GM500, Techniplast) in groups of up to 5 animals per cage, with bedding (corncob, Technilab-BMI) and cage enrichment. Cages were changed weekly, reducing handling of the mice to one handling per week during non-intervention periods.

\section{Atherosclerosis models}

For bone marrow transplantations, male LDLRKO recipients were placed on antibiotic water, containing neomycin (100mg/L; Gibco, Carlsbad, CA, USA) and polymyxin B sulfate (60.000U/L; Gibco) for 7 weeks in total while being fed a chow diet. After one week of antibiotic water, bone marrow isolated from PHD1KO-LDLRKO and PHD3KO-LDLRKO mice and matched LDLRKO controls (hereafter referred to as PHD1KO, PHD3KO and WT respectively), and transplanted $\left(1 \times 10^{7}\right.$ cells/mouse) into lethally irradiated LDLRKO recipients ( $2 \times 6 \mathrm{~Gy}, \mathrm{PHD} 1 \mathrm{n}=20$ control vs. $20 \mathrm{KO}$, PHD3 $\mathrm{n}=18$ control vs. $16 \mathrm{KO}$ ). Mice were left to recover for 6 weeks on chow diet and subsequently placed on HCD ad libitum $(0.25 \%$ cholesterol, SDS 824171) for 8 weeks. PHD2cKO mice and respective controls ( $n=20$ per group) were fed a HCD ad libitum (0.25\% cholesterol, SDS 824171) for 6 weeks, 12 or 20 weeks.

\section{Atherosclerosis quantification and immunohistochemistry}

Mice were euthanized with a pentobarbital overdose $(100 \mathrm{mg} / \mathrm{kg}$ i.p.) and blood was withdrawn via the right ventricle for flow cytometry, absolute white and red blood cell counts (Coulter Ac.T diff, Beckman Coulter) and total cholesterol analysis. Mice were 


\section{5 | Myeloid PHD deficiency in atherosclerosis}

perfused via the left cardiac ventricle with PBS containing sodium nitroprusside $(0.1 \mathrm{mg} / \mathrm{ml}$; Sigma-Aldrich, Seelze, Germany). Aortic arch, root and organs were subsequently excised and fixed in $1 \%$ paraformaldehyde overnight and paraffin-embedded.

Aortic roots and arches were serially sectioned $(4 \mu \mathrm{m})$ and stained with hematoxylin and eosin (H\&E, Sigma) for plaque area and lipid core content quantification. Five consecutive $\mathrm{H} \& \mathrm{E}$ sections at $20 \mu \mathrm{m}$ intervals were analyzed blindly using computerized morphometry (Leica QWin V3, Cambridge, UK) and the sum of the three valves averaged per mouse. Necrotic core was defined as a-cellular and a-nuclear plaque area containing cholesterol clefts, and shown as the percentage necrotic content of the total plaque area. Sections within this $100 \mu \mathrm{m}$ interval were used for remaining immunohistochemical stainings. Antigen retrieval was performed at pH 6 (Dako REAL target retrieval, Dako) (for MAC3, aSMA, and collagen type I), $\mathrm{pH} 9$ (tris-EDTA, made in-house) (for platelet derived growth factor receptor beta (PDGFRB)) or trypsin digestion (for CD31). Mouse atherosclerotic plaques were characterized for macrophage content (MAC3+ area/plaque area, BD Cat. No. 553322), smooth muscle cell content ( $\alpha \mathrm{SMA}+$ area/ plaque area, $\alpha \mathrm{SMA}$ Sigma F3777), PDGFR $\beta$ content (PDGFR $\beta+$ area/ plaque area (C-terminus) (ab32570, clone Y92), and total collagen content (picrosirius red area/plaque area, analyzed with polarized light microscopy, Polyscience 09400). Additionally, adventitial microvessel density (CD31+ microvessels/adventitial area, BD Cat. No. 550274), HIF1 $\alpha$ (Novus Biologicals, NB100-449), Ki67 (Abcam ab15580), and SPP1 (Abcam ab8448) were stained. Specific antigen-antibodies were visualized using appropriate secondary antibodies and diaminobenzamidin (DAB), unless specified otherwise. Fibrous cap thickness was measured in Sirius red stained sections of the brachiocephalic artery (at least three continuous sections) and aortic root (two sections per plaque), where necrotic core size was largest. In the section with largest necrotic core cap thickness was determined at the thinnest part, as the area between the outer edge of the cap and the necrotic core boundary.

In situ apoptosis was quantified on single cell level with TdT-mediated dUTP-X nick end labeling (TUNEL) technology (apoptotic cells $/ \mathrm{mm}^{2}$ plaque area; Roche, 11684795910). FITCdUTP and anti-FITC-HRP labeled antibody binding were visualized using AEC positive substrate chromogen (Dako, K3461). Pictures were converted to pseudo fluorescent images using standard the deconvoluting option (hematoxylin and DAB) in FIJI software, and reassigning hematoxylin and $D A B$ spectra to a blue and red pseudo fluorescent color, respectively.

\section{Flow cytometry and blood variables}

Cells isolated from whole blood were analyzed using flow cytometry ( $n=10$ mice per group). Blood was subjected to erythrocyte lysis. Following specific antibodies were used to detect

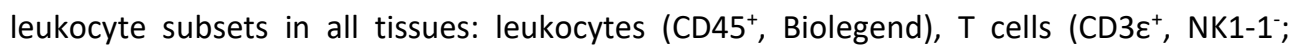


Miltenyi, eBioscience, resp.), B cells (B220+; BD), NK cells (NK1-1 $1^{+}$), granulocytes $\left(C D 11 b^{\text {high }}\right.$ Ly6G ${ }^{\text {high }} ; B D$, eBioscience, resp.) and monocytes (CD11b ${ }^{\text {high }}$ Ly6Glow Ly6C high/intermediate/low; Miltenyi). Data were acquired using a FACS Canto II and analyzed with FACS diva software (BD).

For blood variable analysis, whole blood was diluted 1:10 in Hepes buffer, pH 7.45 (10mM Hepes, $136 \mathrm{mM} \mathrm{NaCl}, 2.7 \mathrm{mM} \mathrm{KCl}, 2 \mathrm{mM} \mathrm{MgCl} 2,0.1 \%$ glucose, $0.1 \% \mathrm{BSA}$ ) and subsequently measured on the XP3000 Sysmex analyzer (Sysmex, Chuo-ku kobe, Japan).

\section{Human tissue collection}

Human plaque tissue collections were used: Maastricht Pathology Tissue Collection (MPTC), ${ }^{38}$ and Biobank of Karolinska Endarterectomies (BiKE) for analysis of protein and mRNA levels using immunohistochemistry, in situ hybridization (ISH), microarrays and western blot analysis. Tissue collection was in line with the Dutch Code for Proper Secondary use of Human Tissue, that is normally considered waste material. This code (https://www.federa.org/codes-conduct) entails an opt-out arrangement and hence tissues were not used in case of objection. The applicability of this code for this study was approved by the Maastricht University hospital (MUMC) local Medical Ethical Committees. Human studies of BiKE are approved by the Ethical Committee of Stockholm and follow the guidelines of the Declaration of Helsinki. All included patients have given their written informed consent.

MPTC samples of human carotid autopsy samples ( $n=38$, mean age 72 years, $64 \%$ men), were used for immunohistochemistry and ISH. Sample represented the following stages of atherosclerosis ( $n=8-10$ per group): intimal thickening, pathological intimal thickening, thick fibrous cap (stable) atheroma, and plaque with intraplaque hemorrhage. ${ }^{38}$

In the BiKE cohort, symptomatic and asymptomatic patients $(n=127)$ undergoing carotid endarterectomy (CEA) were consecutively enrolled in the study and plaques collected for histology and RNA lysates. Transcriptome was analyzed by Affymetrix microarray (Dataset available from Gene Expression Omnibus with accession nr. GSE125771) and interrogated for target genes. Demographics, experimental procedures and array analysis of the BiKE study cohort have been extensively described previously. ${ }^{17,18}$

\section{Human plaque immunohistochemistry and multispectral imaging}

PHD1, 2 and 3 protein expression was assessed in human carotid autopsy samples. PHD1 expression was analyzed by fluorescent immunohistochemistry. After antigen retrieval (target retrieval Dako), slides were incubated overnight with rabbit anti-human PHD1 (1:100 Novus Biologicals NB100-310), followed by biotin-conjugated donkey-anti-rabbit, streptavidin-horseradish peroxidase (HRP) and Cy3 labelled tyramide signal amplification reagent (Perkin Elmer). Nuclei were visualized with DAPI. Following antigen retrieval, PHD2 


\section{5 | Myeloid PHD deficiency in atherosclerosis}

(Novus Biologicals NB100-2219) and CD68 (Dako, M0814) were analyzed by non-fluorescent immunohistochemistry, followed by multispectral imaging to convert into pseudofluorescent images. Multispectral imaging (MSI) was performed to analyze human PHD1 and 2 expression, and PHD-CD68 co-localization. Spectral images were taken between 420$720 \mathrm{~nm}$ (10 nm interval) at a 10x (human) magnification using a Nuance spectral imaging system (Perkin Elmer/Caliper Life Sciences, Hopkinton, MA, USA) mounted on a Leica DM4000 B LED microscope, in case of immunofluorescence by using filters for N21 and A. Single stained sections, one chromogen/fluoro-chrome or counter stain only (DAB, Vector Red, Vector Blue, Dyelight 594, hematoxylin, DAPI), were used to create a spectral library. Computational decomposition of the individual image components was performed using the spectral library and NuanceTM 3.0.2 software. Pseudo-colors were assigned to unmixed images and composite images showing co-localization were generated with the Nuance 3.0 software.

\section{PHD3 In Situ hybridization}

As accurate, reliable PHD3 antibodies are not available at this moment in time, we here use in situ hybridization to locate PHD3 expression in human plaques. PHD3 mRNA expression was determined by in situ hybridization in FFPE, advanced human plaques derived from autopsy, as a specific PHD3 antibody is not available. All solutions used were treated with DEPC and autoclaved before use to eliminate RNAse activity. Sections were deparaffinized, rehydrated and treated with proteinase $\mathrm{K}$ (Invitrogen, 25530-049) to remove endogenous tissue nucleases. PHD3 mRNA was targeted (target sequence (TACATGGTGGGATCCTGCGGATATTTCCAGAGGGGAAATCA

TTCATAGCAGATGTGGAGCCCATTTTTGACAGACTCCTGTTCTTCTGGTCAGATCGTAGGAACCCA CACGAAGTGCAGCCCTCTTACGCAACCAG) by a double-DIG labeled nucleotide probe (TGGTTGCGTAAGAGGGCT) in microRNA ISH Buffer (Exiqon). Hybridization was performed at $55^{\circ} \mathrm{C}$ overnight. After washing off excessive probe with PBS the DIG-labeled probe was targeted by anti-DIG-AP (Roche, ref. 11093274910). Positive signal was visualized with NBT/BCIP AP detection tablets (ref. 11697471001). Cells were counterstained with Fast Red (ScyTek Laboratories, NFS500). Images were converted to pseudo-fluorescence in Fiji.

\section{Cell culture}

Bone marrow was isolated, and cells were cultured for seven days in RPMI-1640 (Gibco with Glutamax, 2g/L glucose) supplemented with $10 \%$ FCS, 100U/ml Penicillin-Streptomycin, and 15\% L929-conditioned medium to generate bone marrow-derived macrophages (BMDM). Macrophage conditioned medium was obtained by culturing differentiated BMDM for $24 \mathrm{~h}$ in DMEM GlutaMAX (Gibco 31966) supplemented with 2\% FCS, 100U/ml PenicillinStreptomycin, and 15\% L929-conditioned medium in either normal oxygen conditions or hypoxia $\left(0.1 \% \mathrm{O}_{2}\right)$. 
Primary vascular smooth muscle cells (SMC) were isolated by enzymatic digestion ( $4 \mathrm{~h}$ at $37^{\circ} \mathrm{C}$ in DMEM with $3 \mathrm{mg} / \mathrm{ml}$ collagenase (Sigma, Zwijndrecht, the Netherlands) and 1 $\mathrm{mg} / \mathrm{ml}$ elastase (Sigma, Zwijndrecht, the Netherlands) from aortas of 5-10 C57/Bl6 mice (after removal of endothelium and adventitia) and cultured in DMEM (Gibco, 31966047) supplemented with $10 \%$ FCS and 100U/ml Penicillin-Streptomycin. NIH/3T3 were cultured in DMEM (Gibco, 31966047) supplemented with 10\% FCS and 100u/ml PenicillinStreptomycin. Before experiments with macrophage conditioned medium, SMCs and $\mathrm{NIH} / 3 \mathrm{T3}$ were starved for $24-48$ hours respectively using DMEM supplemented with $0.1 \%$ FCS.

For gene intervention, cells were incubated with transfection agent Viromer BLUE (Lipocalyx, VB-01LB-01) together with siRNA for HIF1 (5'-GUCACCACAGGACAGUACA-3'), BNIP3(5'-ACCUUCUGAUGAAGAUUUGGAUC-3') and scramble control (5'GCUUAACCCGUAUUGCCUA-3') in a concentration of $25 \mathrm{nM}$ for 8 hours.

\section{Proliferation assay}

Proliferation of SMC and 3T3 fibroblasts in response to macrophage conditioned medium (see above) was measured using CellTiter-glo luminescent cell viability assay (Promega, G7570) to determine ATP content of cells according to manufacturer's protocol. Primary SMCs were seeded in a 96 wells plate and allowed to attach for 24 hours. After starvation for $24 \mathrm{~h}$ in DMEM containing 0.5\% FCS, BMDM-conditioned medium was added to the cells and incubated for 72 hours. Luminescence was measured on a luminometer (Victor3, PerkinElmer) and proliferation calculated as difference on ATP between T0 and T72hrs.

Proliferation of PHD2 WT and cKO BMDMs was also measured on an ACEA xCELLigence (Roche). BMDMs (8x103 cells) were seeded on a gold electrode implemented in a 96 wells plate. Impedance was measured hourly and used to quantify proliferation (slope of impedance increment over time) using RCTA software (version 1.2, Roche).

\section{Migration assay}

Migration stimulation in primary murine SMC by conditioned macrophage medium of WT and PHD2cKO was measured on an ACEA xCELLigence (Roche). SMC were starved in DMEM containing $0.5 \%$ FCS for 24 hours. Upper chambers of ACEA CIM 16 plates (ACEA, 20131122) were coated with $10 \mathrm{ug} / \mathrm{ml}$ collagen $\mathrm{G}$ (Biochrome, L7213) for one hour per side prior to start of the experiment. Subsequently, lower chambers were equilibrated for the respective conditioned mediums and controls. BMDM-conditioned medium contained a final concentration of $1 \%$ FCS and $15 \%$ LCM. SMC ( $4 \times 10^{4}$ cells) were then added to the upper chamber and migration was monitored for $24 \mathrm{~h}$ (hourly measurements), using the slope of the impedance increment over time. 


\section{5 | Myeloid PHD deficiency in atherosclerosis}

\section{Apoptosis}

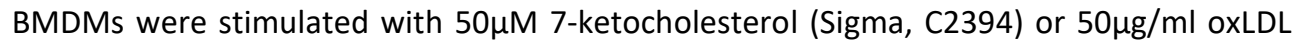
(Isolated as described elsewhere) ${ }^{19}$ for 24 hours to induce apoptosis. After stimulation, nuclei were stained with Hoechst $(15 \mu \mathrm{g} / \mathrm{ml})$ and apoptotic cells with fluorescently labeled AnnexinxA5-FP488 (produced by Prof. Reutelingsperger, Maastricht) for 15 min. Samples were analyzed using a high-throughput, fluorescent reporter system, coupled to automated microscopy (BD Pathway 855 High Content Bioimager). Data was processed with Attovision and BD Diva software.

\section{Efferocytosis}

For efferocytosis analysis in vitro, Jurkat T cells were labeled with calcein-AM $(1 \mu \mathrm{g} / \mathrm{ml}$ Invitrogen) prior to induction of apoptosis by UV irradiation (15 $\min 254 \mathrm{~nm}$, UVS-26, 6W bulb $0.02 \mathrm{~J} / \mathrm{s} / \mathrm{cm}_{2}$ ) and added 3:1 to BMDMs. Macrophages were exposed to $21 \%$ (normoxia) or $0.2 \% \mathrm{O}_{2}$ (hypoxia) during 45 minutes of efferocytosis (Invivo2 1000, Ruskinn technology LTD, Pencoed, UK). After thorough washing, macrophages were dissociated and analyzed using flow cytometry for the percentage of calcein/jurkat ${ }^{+}$macrophages.

\section{Intracellular collagen content}

Intracellular collagen content was measured using CNA35-FITC (Kindly provided by prof. Reutelingsperger, Biochemistry department Maastricht) shown to bind to collagen type I, III and IV. ${ }^{20}$ SMCs and 3T3s were starved in DMEM containing $0.1 \%$ FCS for 24 or 48 hours, respectively. SMCs and 3T3s were subsequently treated with conditioned medium of either PHD2 WT or conditional knock-out macrophages with or without a collagen producing stimulus (TGF- $\beta 1$ ) $(5 \mathrm{ng} / \mathrm{ml}$, Biolegend, 763102) for 72 hours. Cells were fixed in $2 \%$ PFA for 15 min and permeabilized using $0.1 \%$ Triton X-100 in PBS for 15 min. Subsequently, cells were stained for internal collagen content with CNA35-FITC $(1 \mu \mathrm{M})$ and nuclei were stained with Hoechst $(15 \mu \mathrm{g} / \mathrm{ml})$. Samples were analyzed using the BD Pathway 855 High Content Bioimager. Data was processed with Attovision and BD Diva software.

\section{Collagen secretion}

After serum starvation, SMCs and 3T3s were treated with conditioned medium of either WT or PHD2cKO macrophages for 72 hours. Culture medium of SMCs and 3T3s was collected after 72 hours and analyzed using Sircol soluble collagen assay as described by the manufacturer (Biocolor, S1000). In comparable subsequent experiments, TGF- $\beta 1(5 \mathrm{ng} / \mathrm{ml})$ was added to the conditioned medium, or proteins were heat-inactivated $\left(30 \mathrm{~min}, 85^{\circ} \mathrm{C}\right.$ ) prior to addition to $3 \mathrm{~T} 3$ fibroblasts. 


\section{Matrix metalloprotease activity assay}

The functional activity of matrix metalloproteinase (MMPs) was determined using OmniMMP ${ }^{\mathrm{Tm}}$ fluorogenic substrate (Enzo Life Science, BML-P126-0001). The Mca-Pro-LeuGly-Leu-Dpa-Ala-Arg-NH2 substrate incorporates a quencher (N-3-(2,4-dinitrophenyl)-La, $\beta$-diaminopropionyl) and a fluorescent side (7-methoxycoumarin-4-yl)acetyl). Upon MMP cleavage of the quencher fluorescence can be detected. A total of $0.14 * 106$ BMDM (lysed in PBS containing $1 \%$ Trition-X100) was incubated with $180 \mathrm{mM} \mathrm{CaCl}_{2} 65 \mu \mathrm{M}$ OmniMMP substrate at $37^{\circ} \mathrm{C}$ and the fluorescence was detected at an interval of two minutes on a Spectromax (Ex 328nm, Em 393nm, Molecular Devices SPECTRAmax M2). The slope of fluorescence intensity increase was subsequently analyzed.

\section{Western blot}

Whole cell BMDM protein lysate was isolated using RIPA buffer supplemented with protease inhibitors (Roche, 11873580001) and protein concentration determined by BCA assay.

Pre-cast gels (ExpressPlus PAGE gel 8 - 16\%, genscript, M81612) were used to separate 2550 proteins, and transferred to a nitrocellulose membrane. Primary antibodies directed against HIF1 $\alpha$ (Novus Biologicals, NB100-499), HIF2 $\alpha$ (Novus Biologicals, NB100-122), and $\beta$-Actin (Abcam, ab8227), were followed by appropriate HRP-labeled secondary antibody incubation. Signal was developed using SuperSignal West Femto Maximum Sensitivity Substrate (Thermo Fisher scientific, 34095) and visualized using a digital scanner. Band density was quantified with ImageJ, and normalized for total proteins by $\beta$-actin as loading control.

\section{Real time quantitative PCR}

In vitro experiments for gene expression analysis were performed in quadruplicate and repeated twice. Total RNA was isolated by Qiazol (Qiagen, 79306) and transcribed using iSCRIPT (Biorad, 1708891). qPCR analyses were performed with 10 ng cDNA using SYBR green (Biorad) and gene specific primer sets (Eurogentec, Liege, Belgium, Table S1). Two housekeeping genes (cyclophilin, 18S rRNA) were used to normalize differences in mRNA levels between samples.

\section{RNA Sequencing of cultured cells}

For RNA sequencing cells in vitro, RNA was isolated from triplicates of WT and PHD2cKO BMDMs 24 hour after seeding, and from triplicate fibroblasts after 72 hours exposure to WT or PHD2cKO conditioned medium. Bioanalyzer confirmed intact RNA (RNA Integrity number 10 ) for sequencing of $10 \mu \mathrm{g}$ RNA, on the NextSeq 500 system using v 2.5 chemistry, 
at $15 \mathrm{M}$ single reads per sample by the c(Core Facility Genomics of the Medical Faculty Münster.

The gene-level expression of a total of 32,544 genes were quantified, 14,285 genes were retrieved for downstream analysis, 18,259 genes were discarded as lowly expressed genes.

\section{Bioinformatics analysis of bulk RNA sequencing data}

Gene-level expression was quantified using Kallisto with the mouse genome (Mus Musculus GRCm38 assembly). ${ }^{21}$ Principal component analysis was used for exploratory data analysis using the prcomp function in $\mathrm{R}$ (stats package, $\mathrm{R}$ 3.6.1 version). The limma $\mathrm{R}$ package (v3.40.6) was used to test for differential expression between conditions using the empirical Bayes method after voom transformation. ${ }^{22}$ Lowly and non-expressed genes in the experiment were discarded from the analysis using the filterByExpr function (limma) to reduce potential false positives from the multiple testing. Single-sample transcription factor activities were estimated using DoRothEA mouse regulons with $A, B$ and $C$ confidence classes. $^{23,21}$ Similar to differential gene expression analysis, the empirical Bayes method (limma) was also used to test for differential transcription factor activities using the TF activities (normalized enrichment scores) estimated by VIPER method (v.1.18.1). Genes and Transcription factor activities differentially dysregulated with FDR $<0.05$ were considered significant. Pre-ranked Gene-Set Enrichment Analysis (GSEA) was performed using fgsea $R$ package (v1.10.1) on the transcriptome-wide ranking of differential expression by the moderated t-statistics with the hallmark gene set collection from MSigDB and mouse gene sets from MatrisomeDB. ${ }^{24,}{ }^{25}$ Human genes from the hallmark gene set collection were transformed to their orthologs in mouse using the biomaRt service from EnsEMBL. Gene sets with FDR $<0.05$ were considered significantly enriched in the condition. Pathway analysis was performed using PROGENy with the mouse model of pathway footprints of 100 genes $^{20,21}$, and 10,000 gene permutations of the ranking to build a null distribution for statistical estimations of significance. NicheNet ${ }^{27}$ was used for the ligand-receptor analysis of stimulated pro-fibrotic fibroblasts by BMDM PHD2cKO using the differentially overexpressed ligands from BMDMs PHD2cKO ( $p$-value $<0.05$ ), and those target genes that were detected by the leading edge analysis in the significantly enriched MatrisomeDB gene sets from the pre-ranked GSEA in pro-fibrotic fibroblasts.

\section{Single cell RNA sequencing of murine plaques}

PHD2 WT and PHD2 cKO mice ( $n=11$ and 9, respectively) were euthanized with a pentobarbital overdose ( $100 \mathrm{mg} / \mathrm{kg}$ i.p.) after 20 weeks of HCD and blood was withdrawn via the vena cava, followed by PBS perfusion via the left ventricle. The aortic root was subsequently excised and fixed in $4 \%$ paraformaldehyde overnight and paraffin-embedded. 
Aortic arches of either genotype were pooled, sliced in pieces using a scalpel, and enzymatically digested for 15 minutes at $37^{\circ} \mathrm{C}$ using collagenase $B(0.00284 \mathrm{~g} / \mathrm{ml}$, Sigma $110088807001)$, pronase $(0.01 \mathrm{~g} / \mathrm{ml}$, Sigma 10165921001$)$ and DNAse $(0.1 \mathrm{mg} / \mathrm{ml}$, Roche 11284932001). Tissue was filtered through a $70 \mu \mathrm{M}$ strainer and subjected to red blood cell lysis (8.4g NH4CL + 0.84g NaHCO3 in 1 liter H2O, pH 7.2-7.4). All single, DAPI-negative living, cells were sorted on FACS Aria III for SCS. Cell count and viability of sorted cells was confirmed by trypan blue staining, and a total of 12.000 DAPI- plaque cells were loaded on a chromium single cell controller using V2 reagent kit (10X Genomics) to create cDNA sequencing libraries per manufacturers protocol. In short, in reaction vesicles (gel beads in emulsion, GEMs), cells were lysed and barcoded oligonucleotides reverse transcribed before clean-up and cDNA amplification. The Chromium Single-Cell 3' Library Kit was then used to generate indexed sequencing libraries. Sequencing was performed on a Novaseq 6000 system (Illumina) (Table S2).

\section{Bioinformatics analysis of single-cell RNA-sequencing data}

Raw sequencing data (FastQ files) were processed (alignment and gene-level expression quantification) using the CellRanger pipeline (10x Genomics, version 3.1) with the mouse genome (mm10 assembly). Seurat R package (v.3.1.0) was used to perform a standard analysis. ${ }^{26}$ Quality control diagnostics were applied on library sizes, percentage of mitochondrial genes and gene detection coverage of the single cells. Cells with a gene coverage between 500 and 4,000 of genes expressed, and less than $7.5 \%$ of mitochondrial gene expression were retrieved to avoid bad quality cells, such as doublets and dead cells in downstream analysis. After data normalization using log transformation and applying a 10,000 scaling factor, the 2,000 most highly variable genes were selected using the variance stabilizing transformation method implemented in Seurat for each sample. The first twenty principal components from Principal Component Analysis (PCA) applied on this selection of genes were used to find anchors for sample integration of the two conditions PHD2cKO and WT pooled mice, and integrated using Canonical Correlation Analysis implemented in Seurat. ${ }^{26}$ Myeloid leukocytes were identified following two rounds of unsupervised clustering. First, major clusters of cells expressing canonical myeloid leukocytes markers were selected. For this, PCA was applied on the scaled batch-corrected data to extract twenty-five principal components for the unsupervised clustering. Graph-based unsupervised clustering was performed using Shared-Nearest Neighbour algorithm. Louvain method was used to find clusters at resolution 0.1. Cluster of cells with positive expression of PTPRC, Lyz2, and CD68 expression, and absence of other vascular cell markers (CD3, CD19, MYH11, PECAM1) were detected as potential myeloid leukocytes. Second, this large and heterogeneous population of cells was selected for another iteration of unsupervised clustering to find more clusters with a higher resolution (at resolution 0.5 ), following the same workflow as described before. Distinct clusters were annotated based on the markers 


\section{5 | Myeloid PHD deficiency in atherosclerosis}

reported from Zernecke et al 2020, the top 10 marker genes for macrophages, monocytes, dendritic cells and top 10 over-expressed genes for Neutrophils as compared to the rest of cells.51. The main classes of myeloid leukocytes were identified among clusters, including cavity, IFNIC, inflammatory, Trem2-foamy and resident macrophages, mature-DC, moDC, monocytes and neutrophils. In addition, a cluster of proliferating cells was detected using the CellCycleScoring function from Seurat with the ortholog genes in mouse of the cell cycling genes, and another small cluster remained as not assigned (n.a.) due to the nonspecific expression of cell-type markers. Uniform Manifold Approximation and Projection (UMAP) was used to reduce the dimensional space of the twenty five principal components to an embedding of two dimensions for visualization purposes with standard parameters in the Seurat package. Pathway and transcription factor activities were estimated using PROGENy (https://saezlab.github.io/progeny/) and DoRothEA (https://saezlab.github.io/dorothea/) in single-cell data as previously described (pathway footprints of 100 genes and TF regulons of $A, B$ and $C$ confidence classes, respectively). ${ }^{26}$ The enrichment of the PHD2cKO signature expression in single-cell basis was calculated using AUCell R package (v1.6.1) using the top 50 most up-regulated genes in BMDM upon PHD2cKO for the PHD2 functional stratification of plaque-resident macrophages, prior discarding the main molecular players Bnip3 and Spp1 as potentially being defined as part of the signature. Correlations analysis between Hif1a activity, hypoxia response and BMDM PHD2cKO expression signature was performed using Pearson correlation. For differential gene expression analysis, the two clusters of proliferating and n.a. cells were leave out, and the two clusters of resident macrophages found in the unsupervised clustering were merged as a single population of cells. Differential gene expression was performed using Wilcoxon Rank Sum test. P-values from the Wilcoxon Rank Sum tests were adjusted for multiple testing using Bonferroni method for genome-wide multiple testing between two groups, and using FDR method for 2-group individual gene testing among cell types. Differentially expressed genes with adjusted p-values below 0.05 were considered statistically significant. $R$ effect sizes from Wilcoxon Rank Sum test were calculated as $Z$ divided by square root of the total observations. $R$ effect size ranges from -1 to +1 . Positive sign from $r$ effect size relates to up-regulation in PHD2cKO (group 1) as compared to WT condition (group 2). The greater the absolute $r$ value is, the larger the effect size is. $R$ effect size could be interpreted as small ( $r$ between 0.10 and 0.30 ), medium ( $r$ between 0.30 and $0.50)$ and large ( $r>0.50)$.

\section{Data and code availability}

Reproducible code for the transcriptomics analysis is accessible at the GitHub repository: https://github.com/saezlab/Myeloid PHD2 atherogenesis. Raw sequencing data and processed matrices of gene expression from RNA-seq and single-cell RNAseq have been deposited in Gene Expression Omnibus with the accession number: GSE150090. 


\section{Statistical analysis}

All data are presented as mean+SEM, with ${ }^{*}$ p-value $<0.5, * * p$-value $<0.01, * * * p$ value $<0.0001$. All variables were analyzed using independent sample tests and were tested for normal distribution using Shapiro-Wilk normality test. Variables with two groups were compared with Student's t-test or Mann-Whitney rank-sum test. In case of more than two groups, variables were analyzed using one-way ANOVA followed by Bonferroni's Multiple Comparison Test or Kruskal-Wallis rank-sum test, followed by Dunn's post-hoc testing. Correlation analysis was performed using Spearman bivariate correlation analysis (IBM SPSS statistics 22). 


\section{5 | Myeloid PHD deficiency in atherosclerosis}

\section{Results}

\section{PHD1, 2 and 3 in human plaque correlates with plaque inflammation}

Protein (PHD1, 2) and mRNA (PHD1, 2, 3) expression was studied in serial sections of atherosclerotic tissue of human carotid arteries to establish cell-type and plaque stage expression patterns of each isoform. All PHDs were expressed in healthy arteries, and throughout different stages of atherosclerosis (Figure 1A-C). PHD1 was expressed in both CD68 positive and negative regions in plaque and media (Figure 1A), while PHD2 and 3 colocalized predominantly with regions that were CD68-positive in serial sections (Figure 1AB). In line with the importance of inflammation and macrophage function for plaque stability, this suggested PHDs could impact plaque stability. Moreover, we studied expression in the large human cohort BiKE with both healthy and atherosclerotic carotid arteries. Similar to protein expression, mRNA expression patterns of the three isoforms differed in plaques in general, but also when plaques were stratified according to patient symptoms (Figure 1C). The link with inflammation and hypoxic signaling was confirmed by the significant correlations between PHD mRNA and CD68, and HIF1 $\alpha$ and HIF2 $\alpha$ in samples of BiKE cohort subjects (Supplementary (S) Table S3). Thus, expression of PHD proteins in CD68-rich regions in human plaque tissue suggests that PHDs may modulate atherosclerosis via macrophages. 
A
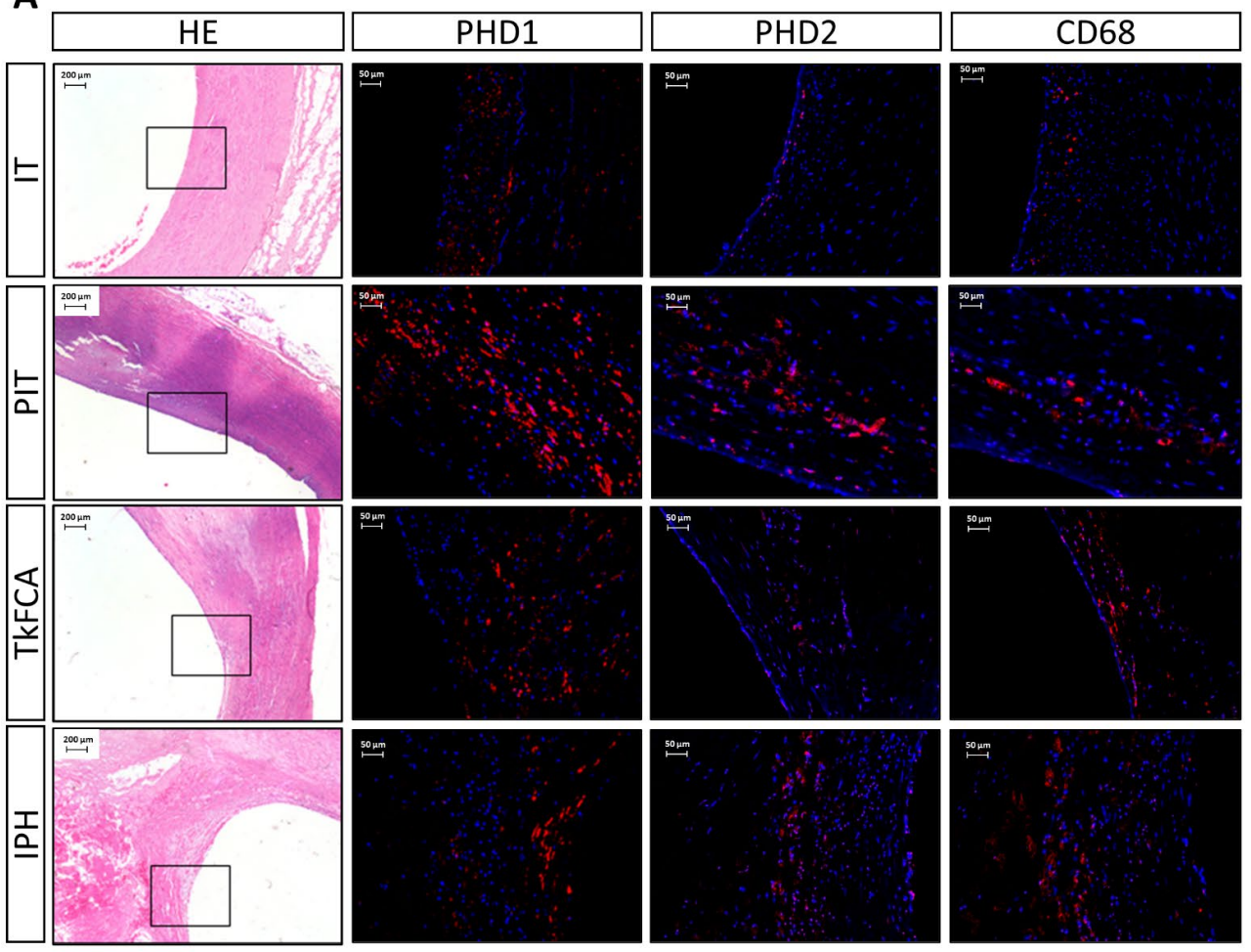

B

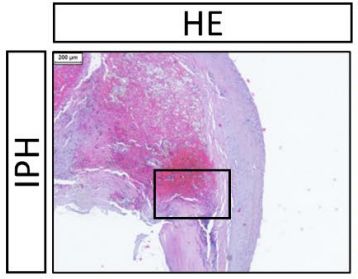

C

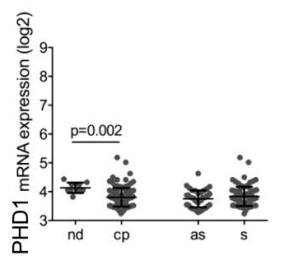

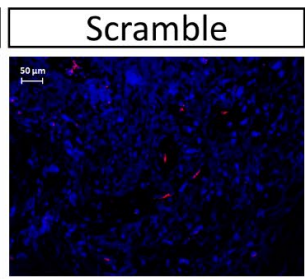
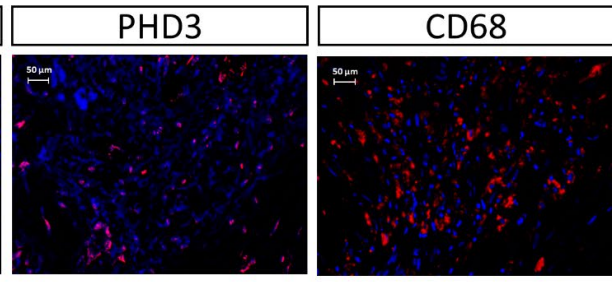

D

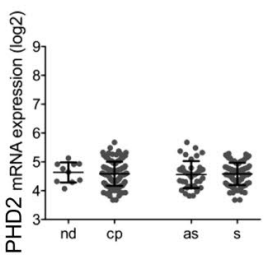

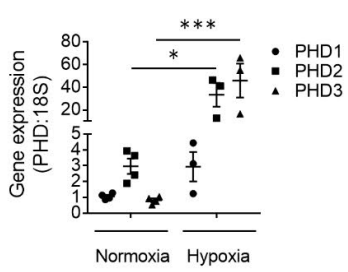

Figure 1. PHDs correlated with in human plaque inflammation (A) Representative pictures of hematoxylin/eosin (HE), PHD1, PHD2 and CD68 in adjacent human carotid plaque sections from different stages(Intimal thickening (IT), Pathological intimal thickening (PIT), Thick fibrous cap atheroma (TKFCA) and Intraplaque hemorrhage (IPH)), (B) Representative pictures of HE, Scramble and PHD3 in situ hybridization, and CD68 immunoreactivity in IPH human carotid plaque, (C) PHD 


\section{5 | Myeloid PHD deficiency in atherosclerosis}

mRNA expression in microarrays of non-diseased arteries ( $n d, n=10)$ and carotid plaques (cp, $n=127$ ) from the BiKE cohort. Carotid plaques were further stratified as asymptomatic (as, $n=40$ ) or symptomatic patients ( $s, n=87$ ), (D) PHD isoforms mRNA expression in bone marrow derived macrophages (BMDMs) measured by quantitative PCR ( $n=3-4$ replicates). Expression relative to $18 \mathrm{~S}$. Statistical analyses were performed using two-way ANOVA, with Bonferroni post-hoc test (C-D). All results show mean $\pm \mathrm{SEM}$. ${ }^{*} \mathrm{P}<0.05,{ }^{* * *} \mathrm{P}<0.001$.

\section{Myeloid PHD1, 2 and 3 deficiency leads to different plaque phenotypes}

The different expression patterns of PHDs in human plaques, and murine BMDMs (Figure 1D), warranted investigation of each isoform in atherogenesis. As only PHD2 is embryonically lethal, PHD2 LysMCre conditional knock-out (KO) mice on a low-density lipoprotein receptor (LDLR) KO background (PHD2cKO) and LysMCre-LDLRKO (WT) were fed a high-cholesterol diet to study atherogenesis. Transplantations of LDLR-KO (WT), PHD1KOLDLR-KO, and PHD3KO-LDLR-KO bone marrow into LDLRKO recipient mice ensured knockout in myeloid cells (Supplementary (S) Figure S2A-C). Both PHD2cKO and PHD3KO led to enhanced plaque size in the aortic root, while PHD3KO additionally showed increased necrotic content (Figure 2A-B). Interestingly, plaques in PHD1KO mice were similar to control, despite a small decrease in plasma cholesterol (Figure S2D). This is surprising, given that whole body PHD1KO and PHD2 hypomorphous mice showed an atheroprotective metabolic phenotype, and smaller plaques compared to control mice. ${ }^{11,12}$ However, PHD1 expression was less pronounced in murine and human macrophages compared to stromal cells, and to macrophage PHD2 and PHD3 (Figure 1). This may explain a preferential stromal, not myeloid, role for PHD1, in line with its role in extrahepatic cholesterol metabolism. ${ }^{11}$ Importantly, no systemic changes occurred in PHD2 and PHD3 deficient mice (Figures S2EF, S3A-C). Similar numbers of circulating neutrophils in all knock-out models and neutrophil presence in PHD2CKO and PHD3KO plaques (Figures S3A.6, B.6, C.6, D-E) suggesting that local vascular effects of macrophages mainly drive their effect on atherogenesis. 


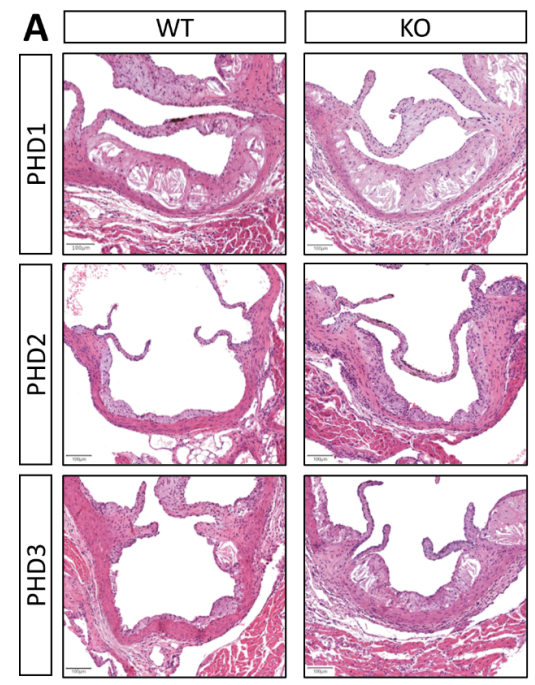

B
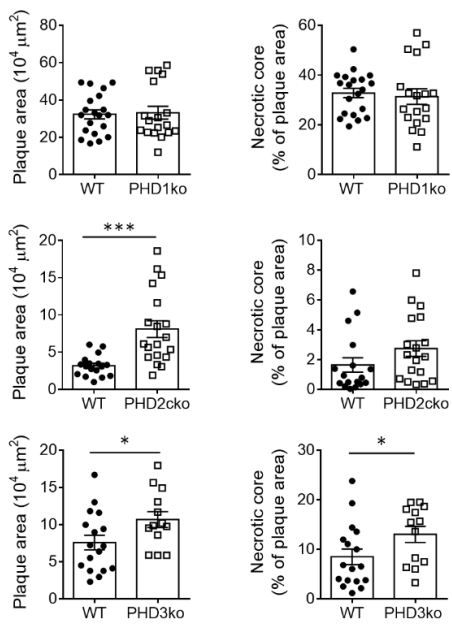

C

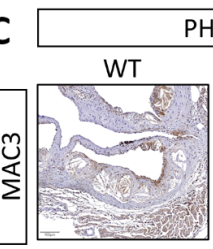
PHD1
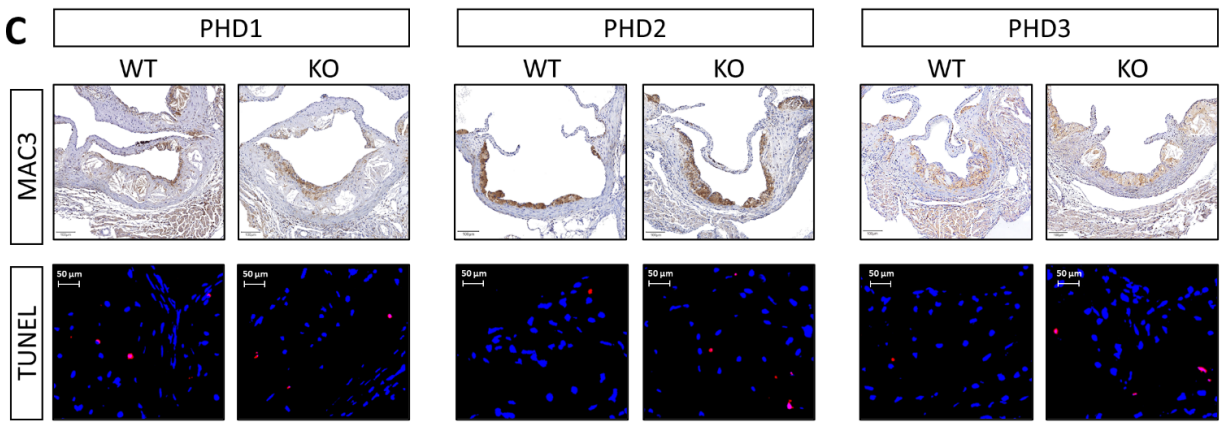

D
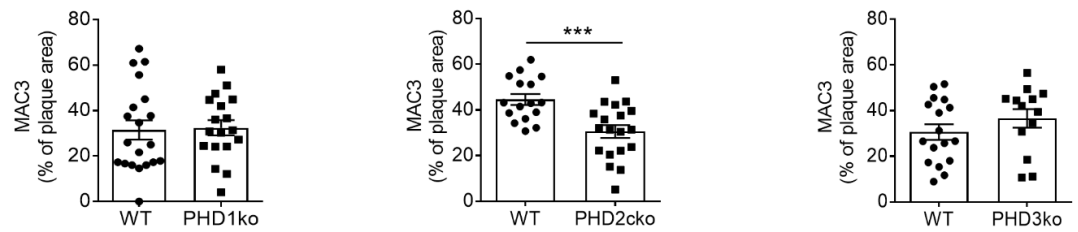

\section{$\mathbf{E}$}
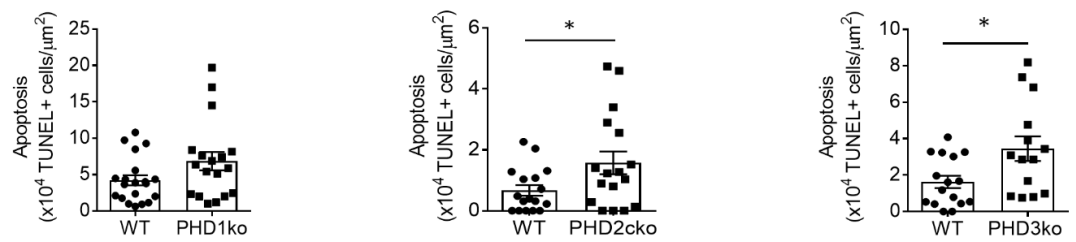

Figure 2. Myeloid PHD2 and PHD3 deficiency aggravated plaque apoptosis (A) Representative HE pictures of aortic root (AR) lesions in PHD knock-outs (KO) and corresponding controls (WT) after 6-8 weeks high-cholesterol diet. (B) Quantification of plaque size and necrotic core size. (C) Representative pictures of MAC3 (brown) and TUNEL (red) staining in ARs of all PHD KO models and corresponding quantification (D,E, respectively). Statistical analyses were performed using a student 


\section{5 | Myeloid PHD deficiency in atherosclerosis}

t-test (B, D, E). All results show mean \pm SEM of $14-20$ mice per group. ${ }^{*} \mathrm{P}<0.05,{ }^{* * *} \mathrm{P}<0.001$. Scale bars $100 \mu \mathrm{m}$.

\section{Myeloid PHD2 and PHD3 deficiency increased macrophage apoptosis}

Local plaque effects in our myeloid PHD knock-outs involved reduced macrophage content in PHD2cKO plaques and larger necrotic cores in PHD3KO plaques, but not PHD1KO plaques (Figure 2C-D). Reduced plaque macrophages and larger necrotic core can both result from increased macrophage apoptosis. Indeed, TUNEL staining revealed enhanced macrophage apoptosis in PHD2cKO and PHD3KO plaques (Figure 2C-E). In line, PHD2cKO and PHD3KO BMDMs, but not PHD1KO BMDMs, showed enhanced 7-ketocholesterol induced annexin Vbased apoptosis in vitro. (Figure 3A-D). PHD2cKO BMDM responded similarly to oxLDL (Figure S4A-B). Uptake of apoptotic cells in vitro was similar between PHD2cKO, PHD3KO, and control cells (Figure S4C-D), suggesting apoptosis alone is underlying enhanced density of apoptotic cells.

\section{Apoptosis in vitro was enhanced via HIF1 $\alpha$ and BNIP3}

Transcriptome-wide mRNA profiling of PHD2cKO BMDMs was performed to study underlying mechanisms (Figures 3E, S5). Pathway analysis using PROGENy ${ }^{27}$, and transcription factor analysis using DoRothEA ${ }^{23}$ in PHD2cKO BMDMs supported hypoxia response activation and HIF1 $\alpha$ transcription factor activity. Hallmarks of cell division, and glycolysis were also upregulated, while inflammatory signaling was diminished (Figures $3 \mathrm{E}$, S5B-D, Tables S4-S5). In contrast, in vitro PHD2cKO showed upregulated expression of both pro- and anti-inflammatory genes (Figure S5E). Interestingly, expression of the proapoptotic HIF target gene BCL2/adenovirus E1B $19 \mathrm{kDa}$ protein-interacting protein 3 (BNIP3) was up-regulated (1.8-fold-change, FDR<0.05; Figures 3E, S5C). Hypoxia culture of control BMDMs, mimicking PHD deficiency, led to increased apoptosis to comparable levels as in PHD-deficient BMDMs. PHD2cKO and PHD3KO BMDM did not show further augmentation of apoptosis upon hypoxia (Figure 3A-D). Increased HIF1 $\alpha$, but not HIF2 $\alpha$ protein levels in PHD2cKO and PHD3KO BMDM and plaques (Figures 3F-G, S6A) was accompanied by enhanced BNIP3 mRNA expression in normoxic and hypoxic PHD2cKO and PHD3KO BMDMs (Figure 3H-J). The causal involvement of HIF1 $\alpha$, and BNIP3 in macrophage apoptosis was confirmed by silencing of HIF1 $\alpha$, or BNIP3 in PHD2cKO and PHD3KO BMDMs, significantly decreasing apoptosis compared to scramble siRNA-treated control cells (Figures 3J-K, S6Bc). 
Myeloid PHD deficiency in atherosclerosis | 5
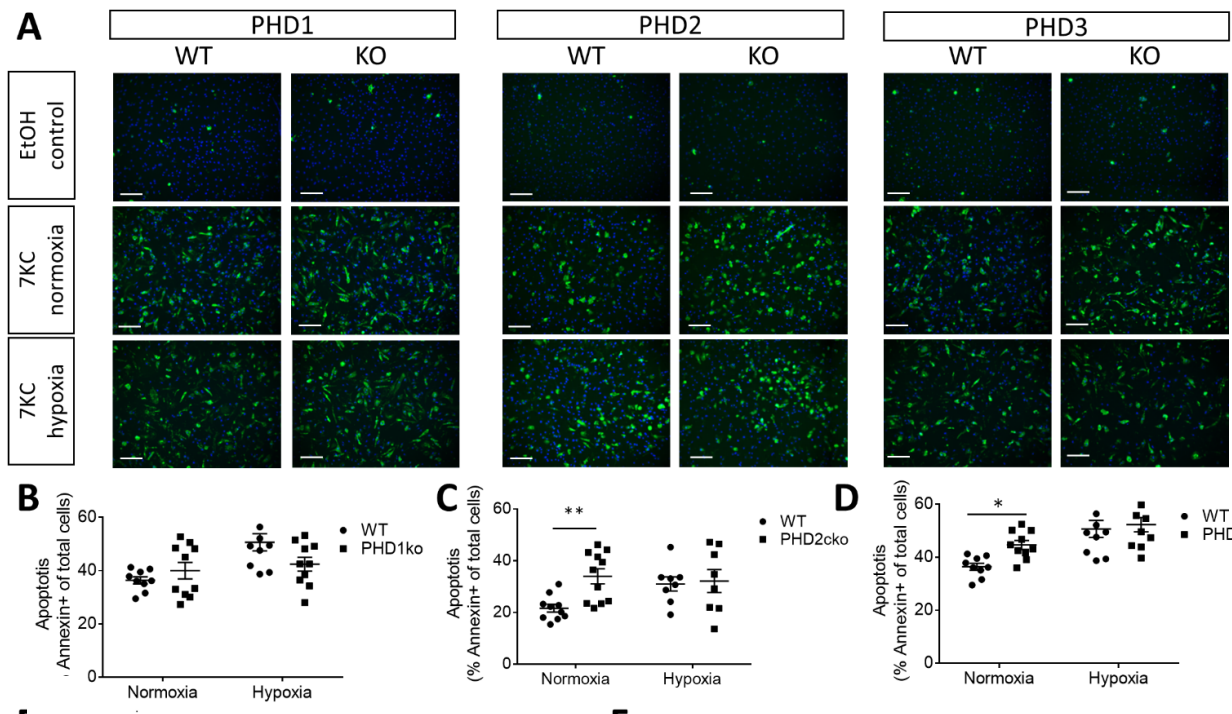

D
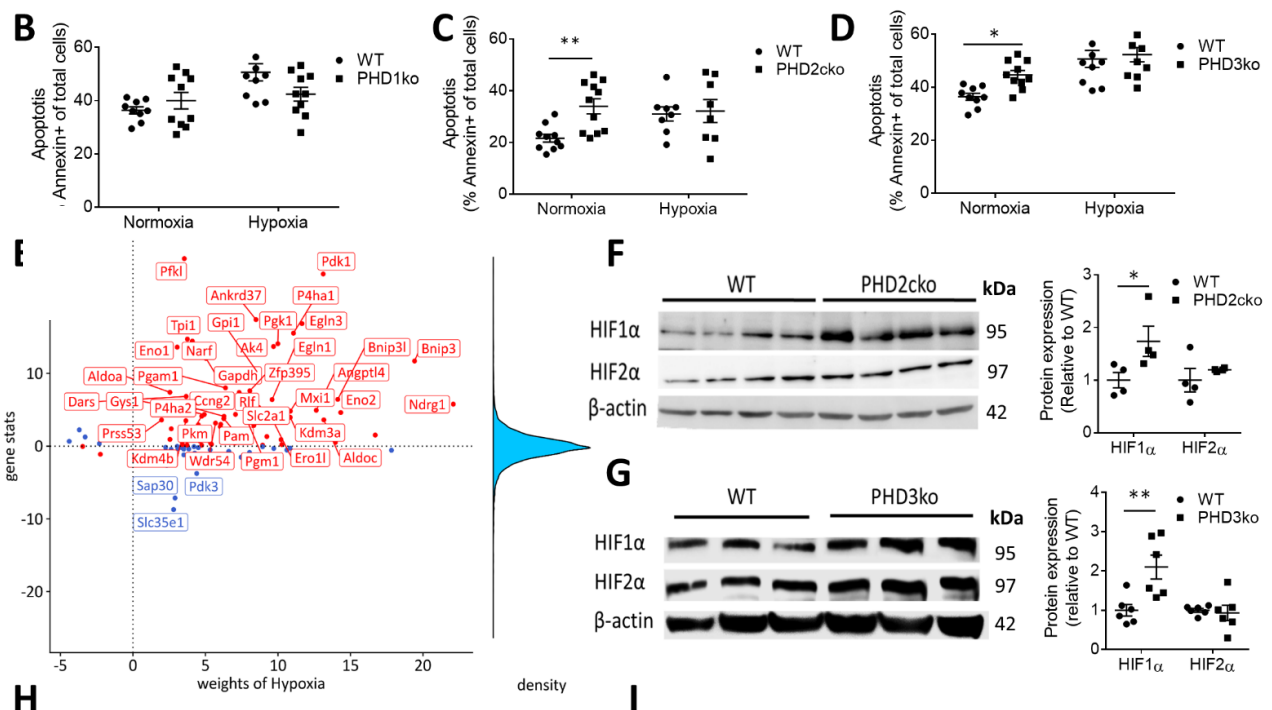

$\mathrm{H}$
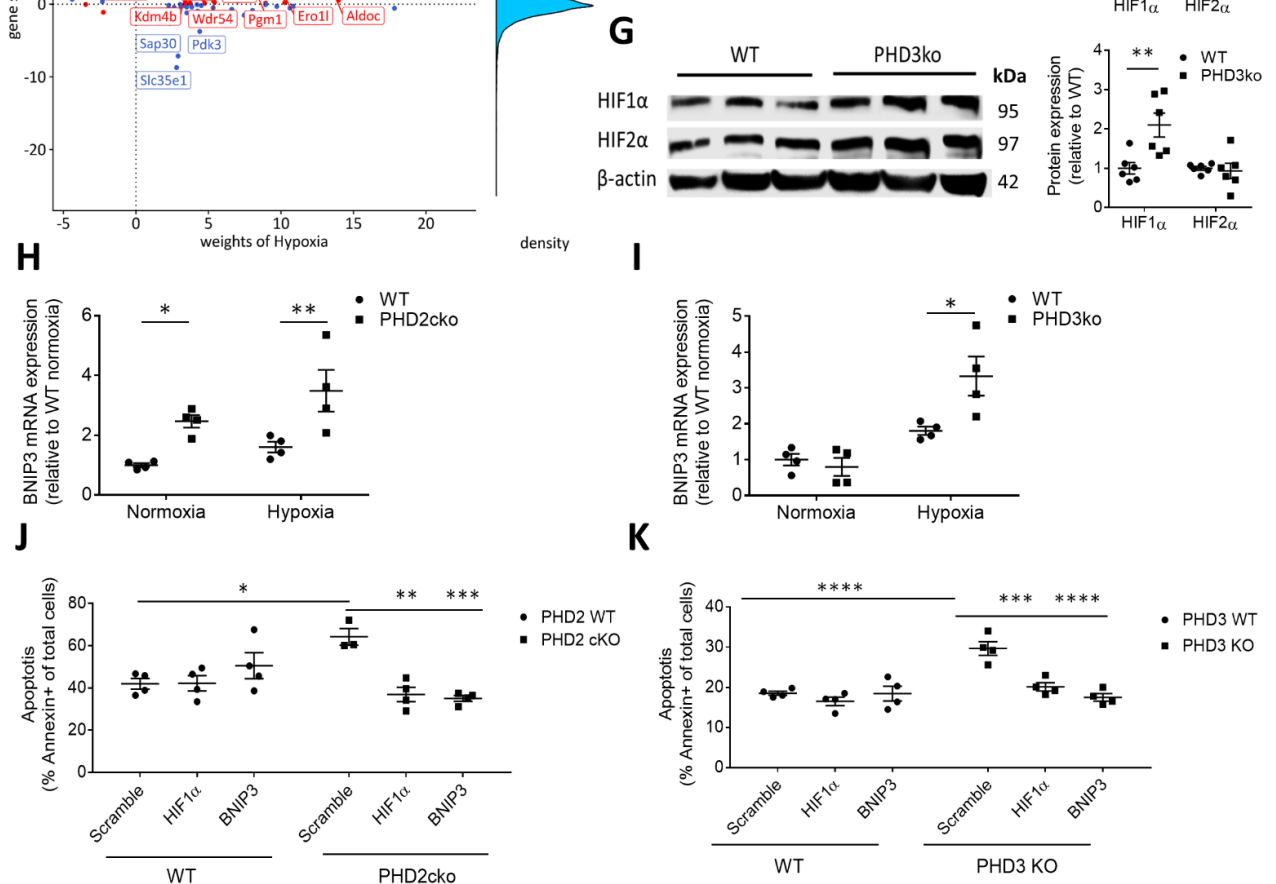

Figure 3. PHD2cKO and PHD3KO enhanced BMDM apoptosis via a HIF1/BNIP3-axis (A) Representative pictures Annexin-A5 (green) stained BMDMs after ethanol (EtOH) or 7-ketocholesterol (7KC) stimulation in normoxia or hypoxia. Nuclei in blue. Bar is $20 \mu \mathrm{m}$. Graphs show 4-11 experiments, 


\section{5 | Myeloid PHD deficiency in atherosclerosis}

which consist of 3-6 biological replicates per experiment (B, C, D) Corresponding quantification of percentage apoptotic cells per isoform. (E) PROGENy footprint of hypoxia response in PHD2cKO macrophages. Top $10 \%$ most dysregulated genes are shown. Red/Blue: positive/negative contribution to activity. (F) Western blot and quantification of HIF1 $\alpha$ and HIF2 $\alpha$ expression in PHD2cKO, (G) PHD3KO and respective control BMDM, normalized for $\beta$-actin. Full blots can be found in Figure S7. (H) BNIP3 mRNA expression in PHD2cKO and (I) PHD3KO BMDM under normoxic and hypoxic conditions. (J) Apoptosis quantification of PHD2CKO,(K) and PHD3KO BMDMs treated with either scramble, HIF1 $\alpha$ or BNIP3 siRNA and 7KC. Experiments show 4-6 biological replicates. Statistical analyses were performed using a two-way ANOVA with Bonferroni post-hoc test (B-D, F-K). All results show mean \pm SEM. ${ }^{*} \mathrm{P}<0.05,{ }^{* * P}<0.01,{ }^{* * *} \mathrm{P}<0.001$.

\section{Single cell sequencing confirmed upregulated HIF1 $\alpha / B N I P 3$ in PHD2cKO plaque macrophages in vivo}

As transcriptional effects of cell-type conditional silencing with partial knock-down can be diluted by other cell types in "bulk" RNA sequencing, single cell RNA sequencing of plaque macrophages was essential to distill the transcriptome of plaque macrophages with PHD2cKO (Figure 4A). The single-cell map shows the landscape of myeloid leukocytes described previously in atherosclerotic plaques (Figure $4 \mathbf{B}$ ) ${ }^{28}$. These myeloid cells were Lyz2-positive, but negative for SMC and endothelial cell markers (Figure 4C). Inflammatory, resident-like, "triggering receptor expressed on myeloid cells-2" (TREM2)-foamy, interferon-inducible cell (IFNIC) and cavity macrophages were distinctly observed and represented in both genotypes (Figure 4D-E, Figure S8A). PHD2cKO plaque macrophages were identified based on the PHD2CKO gene signature, derived from the in vitro transcriptome of PHD2cKO BMDMs. Myeloid cells derived from PHD2cKO plaques presented with a greater PHD2cKO gene signature expression as compared to their matched WT cells (Figure 4F). Next, PHD2cKO cells with signature expression above the 3rd quartile (Q3) from PHD2cKO plaque macrophages (Figure 4F) were compared to WT plaque macrophages below Q3 for differential gene and pathway analysis. This confirmed hypoxia/HIF signaling upon PHD2cKO (Figure 4G), using PROGENy and DoRothEA methods on single cells ${ }^{29}$, and BNIP3 overexpression in PHD2cKO plaque macrophages in vivo (Figure 4H-I). BNIP3 expression was increased across all macrophage subsets. In contrast, BNIP3 was not differentially expressed in PHD2cKO plaque neutrophils with high expression of the PHD2cKO signature (Figure S8B). Further translational impact of this potential new role for BNIP3 in plaque apoptosis was demonstrated by the correlation of BNIP3 mRNA derived from microarrays of whole-mount human carotid plaques with plaque necrotic core (Figure 4J). Together, the PHD2/3-HIF1 $\alpha$-BNIP3 axis likely explains enhanced plaque apoptosis and the ensuing reduction in plaque macrophages in PHD2cKO plaques. 


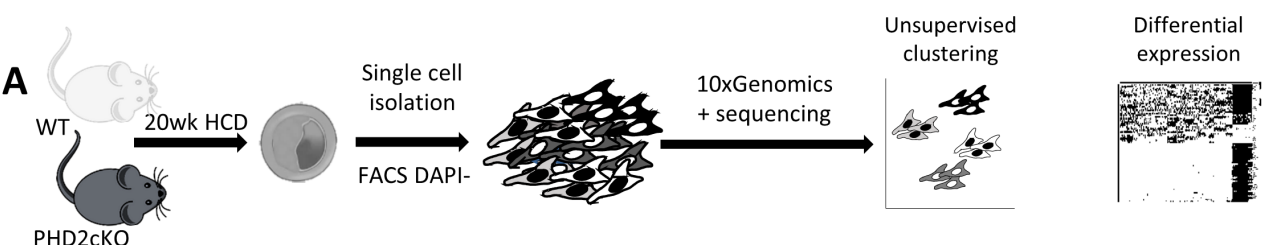

B $_{10}$

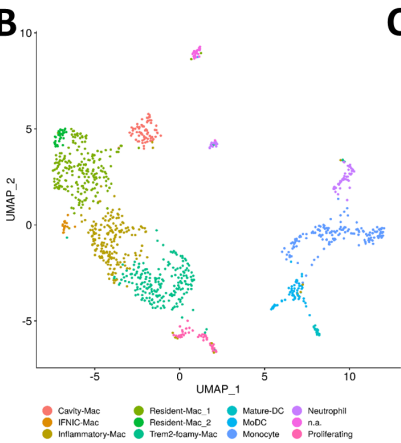

$\mathbf{E}$

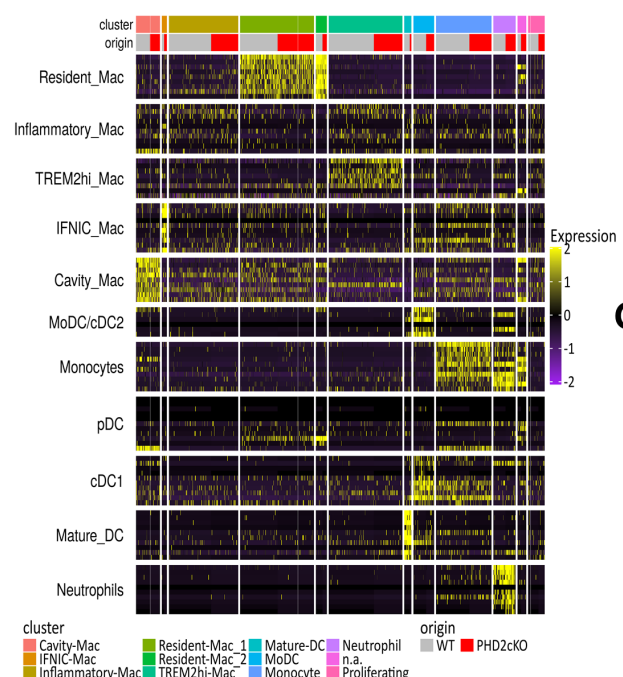

C

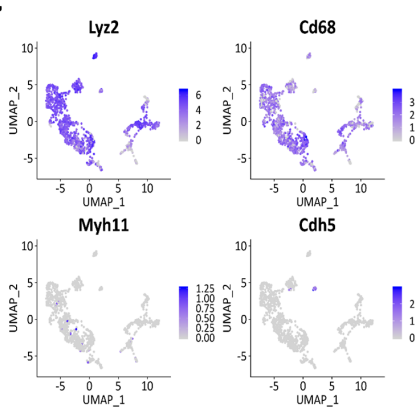

D
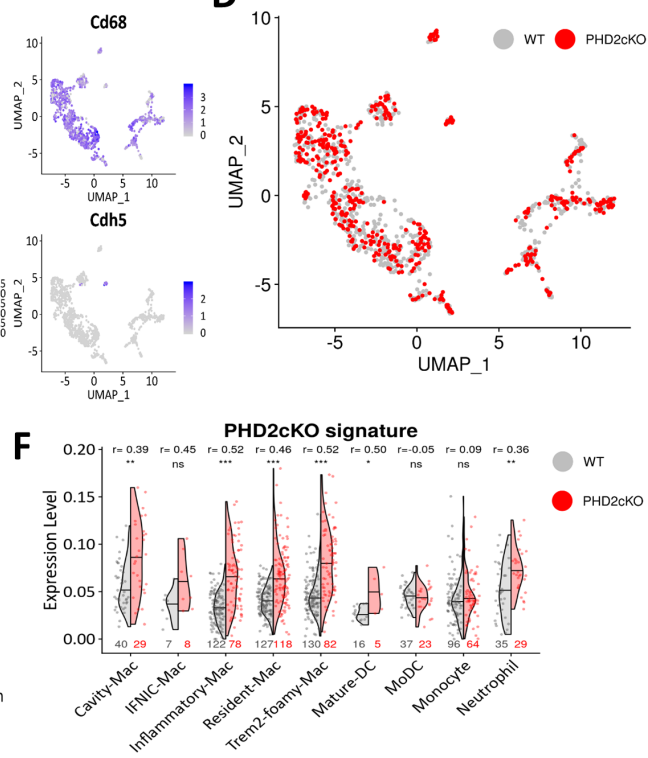

G
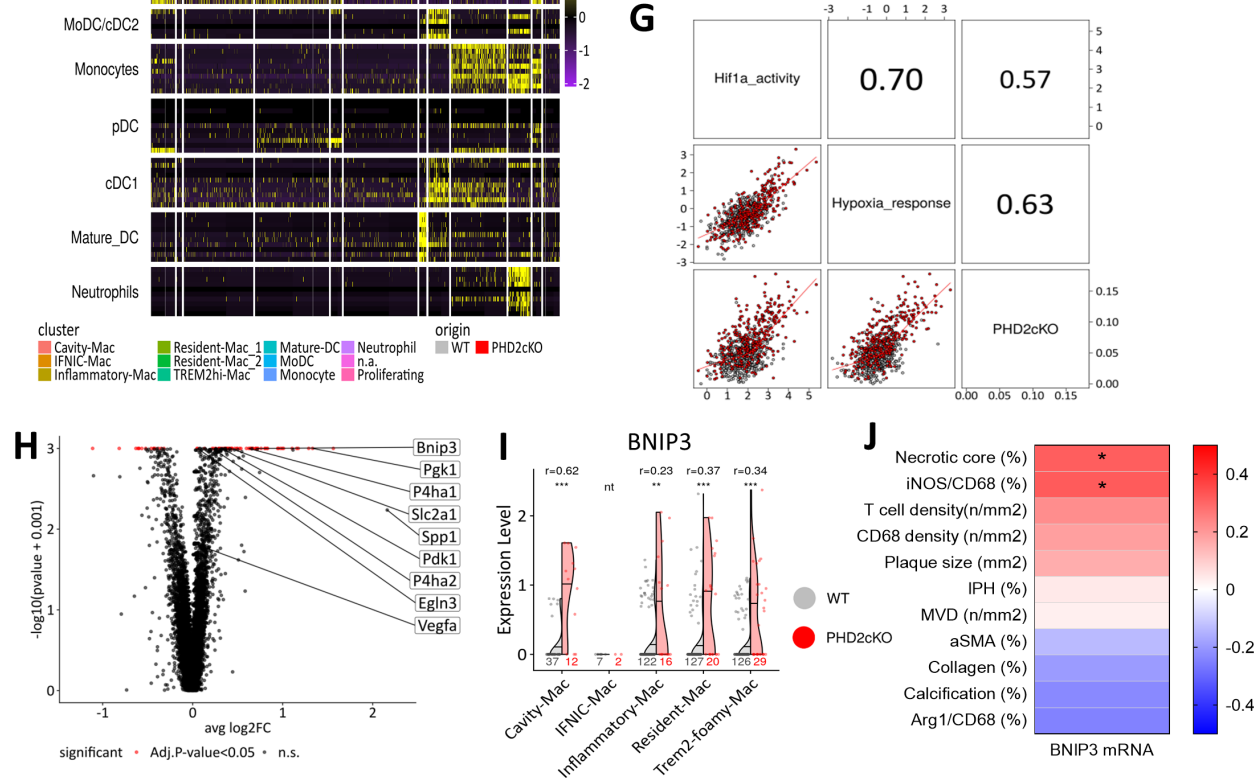

Figure 4. Single cell sequencing of PHD2cKO plaques supported hypoxia signaling and BNIP3 upregulation in vivo (A) Schematic overview single cell sequencing setup, (B) Uniform Manifold 


\section{5 | Myeloid PHD deficiency in atherosclerosis}

Approximation and Project (UMAP) plot of the myeloid leukocytes ( $n=1119)$. Unsupervised clustering resulted in 12 clusters, whose identities were assigned based on cell-specific markers as shown in (E). Each dot represents a single cell, grouped together based on similarities in transcriptome. (C) Same UMAP plot as in (B) showing the absolute expression of canonical markers for myeloid (Lyz2 and CD68), and non-myeloid lineages (Myh11 and Cdh5). (D) Same UMAP plot as in (B) color by biological condition of origin. (E) Heatmap of the top 10 markers from Zernecke et al (2020) of each myeloid leukocyte class across cells. Columns are grouped by the resulting 12 clusters from the unsupervised clustering. Columns are single cells. Rows are marker genes. Gene-level expression was scaled across cells. (F) Violin plots with group $50^{\text {th }}$ quartile (horizontal line) split by condition showing differences in the expression of the BMDM PHD2CKO expression signature among myeloid leukocytes. Each dot represents a cell. ${ }^{* * *} p<0.001,{ }^{* *} p<0.01,{ }^{*} p<0.05$ adjusted $p$-values (FDR method) and $r$ effect sizes from Wilcoxon test. Sample sizes are indicated at the bottom of each violin group. (G) Correlation matrix showing pair-wise Pearson correlation coefficients between PHD2cKO signature and hypoxia and HIF1 $\alpha$ activity scores at a single-cell basis. WT and PHD2cKO cells in grey and red respectively. (H) Volcano plot depicting differentially expressed genes between PHD2cKO and WT plaque macrophages with high ( $>3 Q, n=79$ cells) and low $(<Q 3, n=419$ cells) expression of the BMDM PHD2cKO expression signature, respectively. DEGs with adj. p-value $<0.05$ (Bonferroni method) in red. (I) Violin plots split with group median (horizontal line) by condition showing differences in the Bnip3 expression between PHD2cKO and WT plaque macrophages with high and low expression of the BMDM PHD2cKO expression signature, respectively. Each dot represents a cell. ${ }^{* * *} p<0.001,{ }^{*} p<0.01,{ }^{*} p<0.05$ adjusted $p$-values (FDR method) and $r$ effect sizes from Wilcoxon test. Sample sizes are indicated at the bottom of each violin group. IFNIC-Mac was not tested (nt) due to low sample size $(n<5)$. (J) Heatmap showing FDR adjusted Pearson correlation of BNIP3 mRNA in human carotid plaque segments ( $n=22$ patients) with plaque traits in adjacent sections. ${ }^{*}$ FDR adjusted $p$-value $<0.05$.

\section{PHD2cKO, but not PHD1/3KO, promoted a pro-fibrotic plaque phenotype}

Despite increased apoptosis, larger plaques were observed. In PHD2cKO mice, this was mostly attributed to a 3-fold higher collagen content (Figure 5A), while neither PHD1KO nor PHD3KO mice showed enhanced collagen content (Figure S9). Plaque size and collagen accumulation of PHD2cKO mice were equally enhanced in advanced plaques in the aortic root, and the brachiocephalic artery in a second experiment with mice fed a highcholesterol diet for 12 weeks (Figure S10A-D). In addition to overall collagen accumulation, cap-thickness was increased (Figure 5B). Of note, micro-vessel density was not altered in the adventitial tissue of the aortic root and brachiocephalic artery of PHD2cKO animals, although PHD2 was reported to be the main PHD isoform driving atherogenesis. ${ }^{30}$ Additionally, no plaque micro-vessels were observed in WT or PHD2cKO mice, as expected in mice (Figure S10E-F).

\section{Paracrine signaling by PHD2cKO macrophages enhanced fibroblast collagen secretion}

Collagen secretion by BMDMs themselves was undetectable (figure S10G). Additionally, matrix metalloproteinase activity, and proliferation of PHD2cKO BMDM were unchanged, as measured by two independent assays (Figure 5C-D, data not shown). This hinted towards 
paracrine effects of myeloid PHD2cKO on density or function of collagen-producing cells. Although the current dogma suggests that smooth muscle cells (SMC) are the likely source of plaque collagen production, in vitro and in vivo SMC migration, proliferation, and collagen production remained similar (Figure 5E-I, S11A). Although, the area of aSMApositive mesenchymal cells, i.e. SMCs and fibroblast-like cells, in the plaque was increased in early plaques of PHD2cKO mice, this difference was not maintained at all timepoints and vascular beds (Figure S11B-C). Together these data surprisingly suggested that SMC function and density were not altered by paracrine signaling of PHD2cKO macrophages.

Next, we studied fibroblasts as source of plaque collagen accumulation, since three recent reports indicated fibroblasts involvement in atherosclerosis. ${ }^{31-33}$ Indeed, PHD2cKOconditioned medium induced an almost 3-fold enhanced collagen secretion by 3T3 fibroblasts in vitro, while intracellular collagen content remained unchanged (Figure 5J-K). Fibroblast density seemed unaffected, as mesenchymal marker platelet-derived growth factor receptor $\beta$ (PDGFR $\beta$ ) content of WT and PHD2cKO plaques was similar (Figure S11D), as was fibroblast proliferation and myogenic transition in conditioned medium in vitro (Data not shown; Figure S11E). Thus, changes in fibroblast function in response to PHD2cKO macrophages seemed a likely cause for increased collagen. 

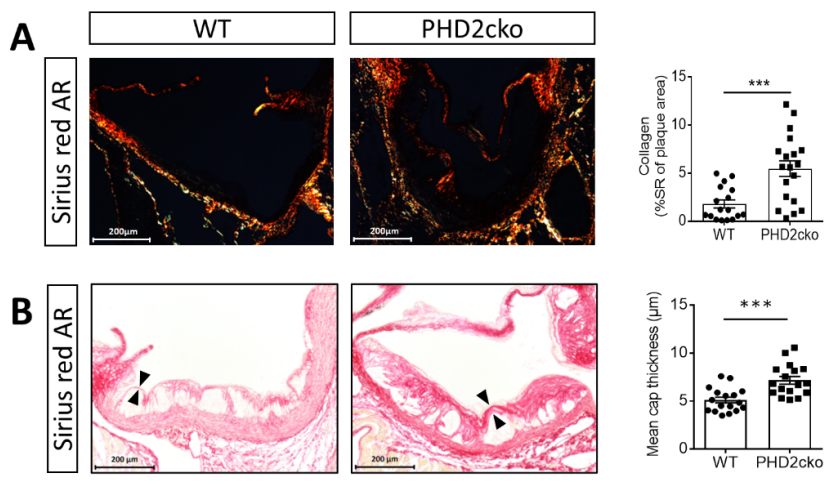

C

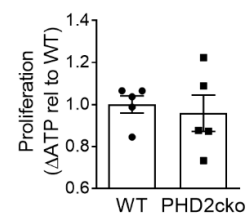

$\mathbf{E}$

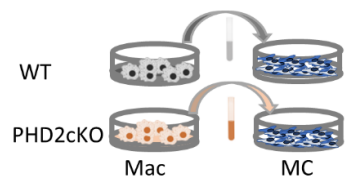

D
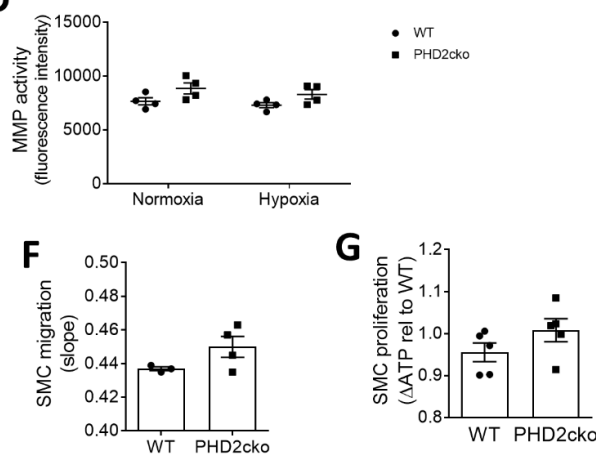
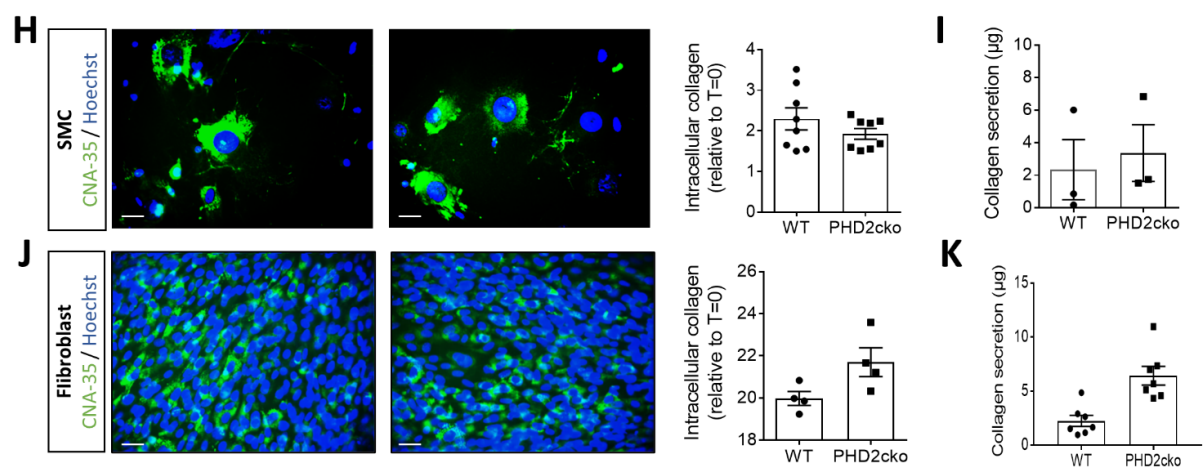

Figure 5. Myeloid PHD2 deficiency triggered plaque fibrosis by enhancing fibroblast collagen secretion via PHD2cKO BMDM paracrine signaling (A) Representative microphotographs of Sirius red collagen content in WT and PHD2cKO mice after 6 weeks HCD. (B) Fibrous cap thickness (arrows) in PHD2cKO and WT mice after 12 weeks HCD. (C) BMDM proliferation (ATP accumulation over 72 hours). (D) MMP activity in WT and PHD2cKO macrophages in normoxia and hypoxia. (E) Schematic overview of conditioned medium transfer from BMDMs to mesenchymal cells (MC). (F) SMC migration after conditioned medium transfer. (G) SMC proliferation (ATP accumulation) 72 hours after conditioned medium transfer. $(\mathbf{H})$ Representative pictures and quantification of internal collagen (CNA-35, green) in SMCs conditioned-medium transfer. Bar is $20 \mu \mathrm{m}$. (I) SMC collagen secretion after conditioned medium transfer. (J) Representative pictures and quantification of internal collagen in 
3 T3 fibroblasts measured by CNA35 (green) after $72 \mathrm{~h}$ conditioned medium transfer. Bar is $20 \mu \mathrm{m}$. (K) 3T3 fibroblast collagen secretion after conditioned medium transfer. In vitro experiments were done with 3-5 technical replicates, and 3-6 biological replicates. In vivo studies include 14-20 mice per group. Statistical analyses were performed using a student t-test (A-C, F-K) or two-way ANOVA, with Bonferroni post-hoc test (D). All results show mean \pm SEM. ${ }^{*} P<0.05,{ }^{* * P}<0.01,{ }^{* * * P}<0.001$. Scale bar $200 \mu \mathrm{m}$.

Mechanistically, the collagen accumulation was hypoxia-dependent, as hypoxia increased collagen production by fibroblasts treated with WT-conditioned medium to similar levels as exposure to PHD2cKO-conditioned medium, without further enhancing collagen production PHD2cKO-conditioned medium treated in fibroblasts (Figure 6A). To further decipher the molecular mechanism, the transcriptome of fibroblasts incubated in vitro with WT or $\mathrm{PDH} 2 \mathrm{cKO}$ medium was interrogated (Figure S12). In line with in vivo and in vitro collagen accumulation, gene-set enrichment analysis showed upregulation of collagen genes (Figure 6B, Table S6), while pathway analysis using PROGENy ${ }^{29}$ showed that the well-known profibrotic Transcription Growth factor beta (TGFß) signaling was down-regulated (Table S7). In line, addition of TGF $\beta$ to WT-conditioned medium did not mimic the enhanced collagen secretion by PHD2cKO-conditioned medium, consistent with similar TGF $\beta$ gene expression by WT and PHD2cKO macrophages (Figure S12C-D).

Nevertheless, a secreted protein seemed responsible, as heat-inactivation of proteins in the conditioned medium ablated collagen accumulation in response to PHD2cKO-conditioned medium (Figure $6 \mathrm{C}$ ). Thus, transcriptomics were analyzed to delineate macrophagefibroblast communication in vitro using Nichenet. ${ }^{34}$ Nichenet identified ligands expressed by macrophages, best predicting the observed changes in signaling response genes expressed by fibroblasts (Figure 6D). The most likely candidate was secreted phosphoprotein 1 (SPP1), also known as osteopontin, which has a well-known correlation with CV events and fibrosis. ${ }^{35-38}$ Indeed, plaque macrophages in single cell analysis also showed a trend to increased SPP1 mRNA expression, which was most pronounced in TREM2-foamy macrophages (Figure 6E-F). Importantly, PHD2cKO plaques showed increased SPP1 protein expression in vivo, which was overlapping but not restricted to MAC3 positive areas (Figure $6 \mathbf{6}-\mathbf{H}$ ). Of note, SPP1 was not detected as differentially expressed genes in plaque neutrophils upon PHD2cKO perturbation (Figure S8B). These results underline that fibroblasts are a major target for paracrine pro-fibrotic signaling mostly by PHD2cKO macrophages, possibly mediated by SPP1 derived from TREM2-foamy macrophages. 
5 | Myeloid PHD deficiency in atherosclerosis
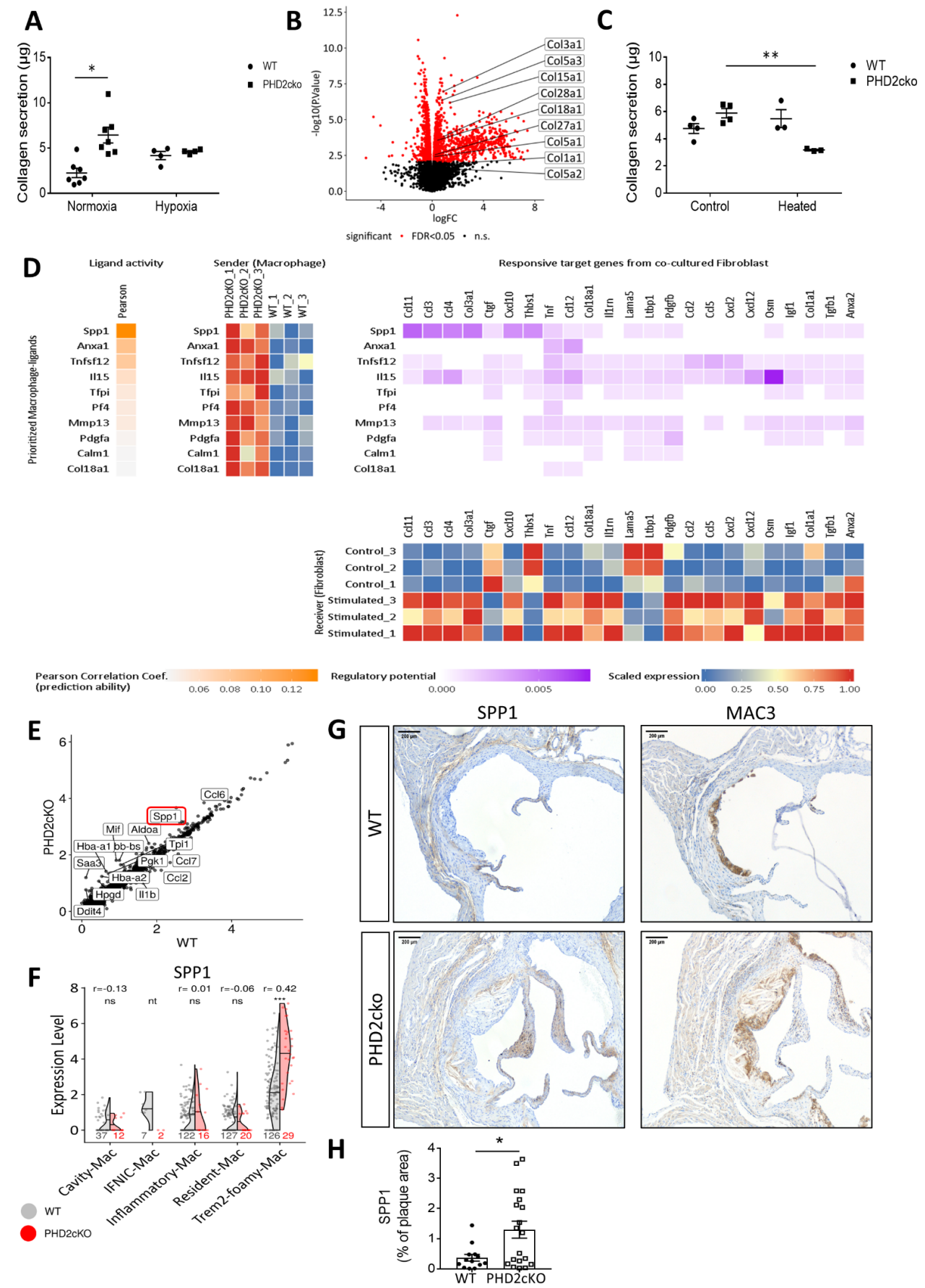

Figure 6. PHD2cKO macrophage-fibroblast communication via SPP1 enhanced collagen accumulation (A) Collagen secretion by 3 T3 fibroblasts after incubation with conditioned medium of normoxic or hypoxic WT and PHD2cko BMDMs. (B) Volcano plot highlighting collagen genes 
upregulated in $3 \mathrm{~T} 3$ fibroblasts exposed to PH2cko-conditioned medium versus WT. (C) Collagen secretion of $3 \mathrm{~T} 3$ fibroblasts upon heat-inactivated conditioned medium exposure from WT- and PHD2cko BMDMs compared to control condition, (D) Nichenet sender-receiver analysis of macrophage-fibroblast communication. BMDM-derived genes (yellow) are ranked by predictive probability of 3 T3 fibroblast transcriptomic changes. Fibroblast genes (purple) are ranked by correlation to the predicted BMDM trigger gene. Heatmaps of gene expression in WT and PHD2cKO BMDM, and WT and PHD2cKO exposed fibroblasts, confirmed differential expression of predicted targets. (E) Scatterplot of log fold change expression between PHD2cKO and Wt macrophages, highlighting SPP1 as one of the most up-regulated genes. (F) Violin plots with group $50^{\text {th }}$ quartile (horizontal line) split by genotype showing differences in the Spp1 expression between PHD2cko (orange) and WT (grey) plaque macrophages with high ( $>$ Q3) and low expression (<Q3) of the BMDMderived PHD2cKO signature, respectively. Each dot represents a cell. ${ }^{* * *} \mathrm{p}<0.001,{ }^{* *} \mathrm{p}<0.01,{ }^{*} \mathrm{p}<0.05$ adjusted $p$-values (FDR method) and $r$ effect sizes from Wilcoxon test. Sample sizes are indicated at the bottom of each violin group. IFNIC-Mac was not tested $(n t)$ due to low sample size $(n<5)$. (G) Representative images of SPP1 and MAC3 immunoreactivity in WT and PDH2cKO ARs after 6 weeks of HCD. (H) Quantification of SPP1 immunoreactivity in WT and PHD2cKO ARs. In vitro experiments done with 3-5 technical replicates, and repeated 3-6 times. In vivo studies include 16-20 mice per group. Statistical analyses were performed using a two-way ANOVA, with Bonferroni post-hoc test (A, C) or a student t-test $(\mathbf{H})$. All results show mean $\pm \mathrm{SEM}$. ${ }^{*} \mathrm{P}<0.05,{ }^{* * * \mathrm{P}<0.001 .}$ 


\section{5 | Myeloid PHD deficiency in atherosclerosis}

\section{Discussion}

In this study, the effect of PHD-dependent pathways on myeloid hypoxic signaling in the atherosclerotic plaque was assessed. Our study indicates that myeloid deficiency of PHD2 and PHD3, but not PHD1, increased plaque size and altered plaque phenotype via intracellular and/or paracrine signaling. Intracellular signaling in PHD2cKO and PHD3KO increased macrophage apoptosis in a HIF1 $\alpha$ /BNIP3 axis dependent manner, an effect observed across macrophage subsets. Interestingly, our studies unveiled paracrine signaling of PHD2cKO, TREM2-foamy macrophages to fibroblasts, inducing the latter towards a profibrotic phenotype.

The pro-apoptotic effect of PHD2cKO in macrophages seemed dependent on the lipid milieu, as PHD2cKO BMDM on a normolipidemic background did not show changes in apoptosis upon incubation with non-lipid apoptotic stimuli. ${ }^{39}$ Nevertheless, a microarray of these PHD2cKO BMDM also showed upregulated BNIP3 expression, ${ }^{39}$ in line with our findings. Although the relationship between HIF signaling and BNIP3 activation is known from carcinogenesis, the role of BNIP3 in the context of atherosclerosis has not been described before. Here we show expression of BNIP3 mRNA associated with human plaque necrosis and pro-inflammatory macrophages. Future studies are warranted to determine if BNIP3 deficiency blocks atherogenesis, and if inhibition is a viable new therapeutic option.

Despite increased apoptosis, plaques in PHD3KO and PHD2cKO mice were not smaller, as one might expect in early lesions and intact clearance of apoptotic cells. ${ }^{40}$ Additionally, in PHD3KO mice macrophage surface area was even larger (data not shown), while content (\% surface area) was unchanged. This may be explained by increased macrophage adhesion and/or migration, as we observed similar proliferation, lipid uptake, and -efflux in vitro (data not shown), and no increased size of other plaque components. Indeed, HIF1 has been shown to induce monocyte adhesion upon acute hypoxia ${ }^{41}$. Macrophage migration upon PHD3KO was shown unaffected by Beneke et al. ${ }^{42}$ Nevertheless, enhanced necrotic core content was in line with stimulation of apoptosis.

In contrast, myeloid PHD2 deficiency enhanced plaque fibrosis may readily explain increased plaque size. Unlike recent papers supporting transition of macrophages into a collagen-producing, fibroblast-like subtype, ${ }^{43,44}$ we uncovered a paracrine, pro-fibrotic effect on fibroblasts. The prevailing dogma is that SMCs are the dominant source of matrix production in atherosclerosis, yet we did not observe any changes in SMC function upon PHD2cKO conditioned medium transfer. Much less is known about the function of fibroblasts in atherosclerosis. Two recent studies pointed at the contribution of fibroblastlike cells to matrix production in atherosclerosis, ${ }^{31,32}$ while only in 2019 single cell sequencing described two fibroblast types and an intermediate modulated SMC in human and murine atherosclerosis. ${ }^{33}$ Here, we show evidence for paracrine signaling of 
macrophages to fibroblasts likely mediated by SPP1. SPP1 is a matricellular protein expressed by most vascular cells, and its function depends on disease state and duration. ${ }^{36}$ This likely explains divergent data of reduced collagen in smaller plaques of atherogenic, normoglycemic SPP1KO mice, contrasting with enhanced vascular calcification in advanced stages of diabetic mice. ${ }^{38,45}$ Despite these ambiguous data on its mode of action, a strong body of experimental and epidemiological evidence showed that both circulating and plaque levels of SPP1 are associated with plaque inflammation, instability and cardiovascular events, ${ }^{35}$ suggesting that combined effects of PHD2cKO on macrophage apoptosis and fibroblasts may aggravate atherosclerosis. Interestingly, SPP1 is known for its anti-apoptotic properties in numerous cell types. ${ }^{46,47}$ One could speculate that antiapoptotic macrophage signaling via SPP1 would lead to paracrine, pro-fibrotic signaling. Interestingly, pro-apoptotic BNIP3 mRNA was enhanced across all macrophage subsets, while SPP1 upregulation was most pronounced in TREM2-foamy macrophages. This suggests a new function of TREM2-foamy macrophages, in addition to their putative role related to lipid uptake, lysosomal digestion and lower inflammatory profile. ${ }^{28}$ Interestingly, TREM2-foamy plaque macrophages express high levels of MMPs, suggesting matrix degradation. Yet, our data suggest a pro-fibrotic potential of TREM2-foamy plaque macrophages, in line with fibrosis-association of TREM2+ liver macrophages. ${ }^{48}$ Our findings underline the functional relevance of biological insights derived from studying cell type subsets with single cell sequencing.

To give further perspective of our findings on the impact of hypoxia, and HIF-dependent and HIF-independent effects of PHDs on atherogenesis, we will briefly discuss fibrosis, apoptosis and cholesterol hemostasis. In general, hypoxia and transient HIF activation are known to drive fibrosis and tissue repair, however, chronic hypoxia can also lead to excessive scar formation in lung and liver. ${ }^{49,50}$ In the context of atherosclerosis we show here that PHD2cKO and exacerbated hypoxic signaling also leads to excessive fibrosis in plaques. Chronic application of environmental hypoxia has also been linked to aggravated plaque development in ApoE ko mice, but fibrosis was not measured.$^{51}$ Reversal of hypoxia by admission of high oxygen gas, carbogen, in Ldlr ko mice did not affect collagen accumulation or induction of apoptosis, but improved efferocytosis and necrotic core formation. ${ }^{3}$ Moreover, two studies reporting on HIF1 $\alpha$-ko bone marrow transplantations show either no effect on atherogenesis, or ameliorated plaque size, necrosis and apoptosis, while fibrosis and macrophage content was not changed. ${ }^{8,52}$ This underscores divergence of cell-specific and systemic effects, and the possibility of HIF-independent effects of PHDs. More cell-specific effects of hypoxia can be seen in regards to apoptosis. In neutrophils of normocholesterolemic mice, hypoxia led to a decrease of pro-apoptotic proteins, while in macrophages hypoxia induced apoptosis, and HIF1 deficiency reduced apoptosis. ${ }^{8}$ Strikingly both effects are achieved via NFKB signaling. ${ }^{53,54}$ Lastly, PHD1 and 2 whole-body KO mice showed strong amelioration of hypercholesterolemia, while the effect of PHD3 stromal cell 


\section{5 | Myeloid PHD deficiency in atherosclerosis}

is not yet known. ${ }^{11,12}$ As PHD1, 2 and 3 myeloid deficiency did not alter cholesterol levels, this suggests that stromal cells are responsible for PHD-dependent effects on cholesterol biology. Indeed, intestine and liver were proposed to govern these responses in PHD1KO and PHD2KO mice respectively, although organ-specific PHDKO would be needed to fully prove this. At the very least, the current study shows that PHD1 myeloid cells do not affect vascular biology in hyperlipidemic conditions. Together, this all emphasizes the enormous complexity of cell-type specific, temporal, and lipidemia-related reactions to low oxygen tensions and HIF signaling.

The presence of both destabilizing, pro-apoptotic versus stabilizing, pro-fibrotic effects in our PHD2cko model depict the complex role of hypoxia and/or HIF signaling in plaque development. It can be argued that larger plaques are never a good feature. However, one could claim that fibrosis significantly increases plaque stability. When extrapolating these findings to humans, that would mean decreased possibility of plaque rupture and thus clinical manifestations. Currently, various PHD inhibitors are approved for treatment of anemia upon CKD. PHD inhibition in mice and man unexpectedly lowered cholesterol, as was shown by our group and others. ${ }^{12,55,56}$ Notably, most inhibitors show PHD2 selectivity, ${ }^{57,58}$ and treatment of up to four years is potentially long enough to affect plaque phenotype. Our current study shows development of both beneficial, and detrimental plaque traits related to PHD2 inhibition. These aspects should be taken into account when prescribing PHD inhibitors to CKD patients, who are already at risk for CVD development.

The current comparison between PHD isoforms is limited as we made use of two different models to assess the effects of PHD knock-down (KD) in myeloid cells: cell type conditional LysMcre-mediated knockdown of PHD2, and bone marrow transplantations of PHD1 and PHD3. Both models are however well-established models to assess myeloid specific KD effects. Irradiation could have influenced smooth muscle cell contribution to plaque formation and fibrosis, as suggested by Newman et al. ${ }^{59}$ Thus, we cannot exclude that plaque composition could have been influenced in our PHD1 and PHD3 knock-out models, abolishing or enhancing possible fibrotic effects. A second limitation of our models is that PHDs were silenced in both macrophages and neutrophils, while our current study mainly focused on macrophages. However, neutrophil numbers were unchanged both in the blood and in our single cell RNA seq dataset of PHD2 WT and cKO plaques, and similar neutrophil content was observed in PHD3KO plaques. In addition, neither BNIP3 nor SPP1 were significantly upregulated in neutrophils of PHD2cKO plaques analyzed by single cell sequencing. Hence, this supports that macrophages are predominantly responsible for the observed plaque phenotypes.

To conclude, we show that myeloid PHD isoforms have diverging roles in atherosclerosis. Myeloid PHD2CKO and PDH3KO mediate this via an exaggerated HIF-BNIP3 apoptotic 
Myeloid PHD deficiency in atherosclerosis | 5 pathway across all plaque macrophage subsets, while SPP1 in the subset of TREM2-foamy macrophages likely enhance pro-fibrotic paracrine signaling to fibroblasts. 


\section{5 | Myeloid PHD deficiency in atherosclerosis}

\section{Acknowledgements}

The authors would like to thank A. Jaminon, R. van Gorp, N. Decker, and J. Cosemans, the core facility Genomics of the Medical Faculty Münster, A. Dugourd for the technical support in the pathway analysis, and the core facilities for flow cytometry (Flow Cytometry Facility) in Aachen and Maastricht for their technical assistance.

\section{Author contributions}

KvK, JAFD, TLT and JCS conceived and designed the study. KvK, JAFD, TLT, EM, JdB and HJ performed experiments and/or analyzed data. JPP and JSR analyzed bioinformatically the data. CK and RK provided the $10 X$ Genomics single cell V2 reagents kit and fluidics machine. PC provided the PHD mouse models. MG performed pathological analysis on murine plaques. Human BiKE cohort design and acquisition was done by LM and UH, MaasHPS cohort design and acquisition was done by BMEM, JCS and EALB. CPMR and LS provided AnXA5-FP488 for apoptosis detection in vitro. JAFD, KvK and JCS wrote the main manuscript text. KvK, JAFD, JPP, TLT and JCS prepared the figures. All authors reviewed and approved the manuscript.

\section{Funding sources}

This work was supported by: VENI and VIDI fellowship of the Dutch Organization for scientific research (to JCS 016.116.017, 0.16.186.364), a dr. Dekker senior postdoc fellowship of the Dutch Heart Foundation (to JCS, 2016T060), a Fondation Leducq transatlantic network of excellence (Autophagy in CVD to JCS) and two CARIM PhD fellowships (to TLT 2010, and HS BAFTA for 'talented future PhD candidates' to JAFD, 2018), and the JRC for Computational Biomedicine (JSR), which is partially funded by Bayer AG.

\section{Disclosures}

None. 


\section{References}

1. Winkels $\mathrm{H}$, Ehinger $\mathrm{E}$, Vassallo $\mathrm{M}$, et al. Atlas of the Immune Cell Repertoire in Mouse Atherosclerosis Defined by Single-Cell RNA-Sequencing and Mass Cytometry. Circ Res. 2018;122:1675-1688.

2. Cochain C, Vafadarnejad E, Arampatzi P, et al. Single-Cell RNA-Seq Reveals the Transcriptional Landscape and Heterogeneity of Aortic Macrophages in Murine Atherosclerosis. Circ Res. 2018;122:1661-1674.

3. Marsch E, Theelen TL, Demandt JA, et al. Reversal of hypoxia in murine atherosclerosis prevents necrotic core expansion by enhancing efferocytosis. Arterioscler Thromb Vasc Biol. 2014;34:2545-53.

4. Sluimer JC, Gasc JM, van Wanroij JL, et al. Hypoxia, hypoxia-inducible transcription factor, and macrophages in human atherosclerotic plaques are correlated with intraplaque angiogenesis. J Am Coll Cardiol. 2008;51:1258-65.

5. Kaelin WG, Jr. and Ratcliffe PJ. Oxygen sensing by metazoans: the central role of the HIF hydroxylase pathway. Mol Cell. 2008;30:393-402.

6. Bishop T and Ratcliffe PJ. HIF hydroxylase pathways in cardiovascular physiology and medicine. Circ Res. 2015;117:65-79.

7. Imtiyaz $\mathrm{HZ}$ and Simon MC. Hypoxia-inducible factors as essential regulators of inflammation. Curr Top Microbiol Immunol. 2010;345:105-20.

8. Aarup A, Pedersen TX, Junker N, et al. Hypoxia-Inducible Factor-1alpha Expression in Macrophages Promotes Development of Atherosclerosis. Arterioscler Thromb Vasc Biol. 2016;36:1782-90.

9. Ben-Shoshan J, Afek A, Maysel-Auslender S, et al. HIF-1alpha overexpression and experimental murine atherosclerosis. Arterioscler Thromb Vasc Biol. 2009;29:665-70.

10. Zheng $X$, Zhai $B$, Koivunen $P$, et al. Prolyl hydroxylation by EgIN2 destabilizes FOXO3a by blocking its interaction with the USP9x deubiquitinase. Genes Dev. 2014;28:1429-44.

11. Marsch E, Demandt JA, Theelen TL, et al. Deficiency of the oxygen sensor prolyl hydroxylase 1 attenuates hypercholesterolaemia, atherosclerosis, and hyperglycaemia. Eur Heart J. 2016;37:2993-2997.

12. Rahtu-Korpela L, Maatta J, Dimova EY, et al. Hypoxia-Inducible Factor-Prolyl 4Hydroxylase-2 Inhibition Protects Against Development of Atherosclerosis. Arterioscler Thromb Vasc Biol. 2016.

13. Aragones J, Schneider M, Van Geyte K, et al. Deficiency or inhibition of oxygen sensor Phd1 induces hypoxia tolerance by reprogramming basal metabolism. Nat Genet. 2008;40:170-80.

14. Bishop T, Gallagher D, Pascual A, et al. Abnormal sympathoadrenal development and systemic hypotension in PHD3-/- mice. Mol Cell Biol. 2008;28:3386-400.

15. Takeda Y, Costa S, Delamarre E, et al. Macrophage skewing by Phd2 haplodeficiency prevents ischaemia by inducing arteriogenesis. Nature. 2011;479:122-6.

16. Clausen BE, Burkhardt C, Reith $\mathrm{W}$, et al. Conditional gene targeting in macrophages and granulocytes using LysMcre mice. Transgenic Res. 1999;8:265-77.

17. Matic LP, Jesus Iglesias $M$, Vesterlund $M$, et al. Novel Multiomics Profiling of Human Carotid Atherosclerotic Plaques and Plasma Reveals Biliverdin Reductase B as a Marker of Intraplaque Hemorrhage. JACC Basic Trans/ Sci. 2018;3:464-480. 


\section{5 | Myeloid PHD deficiency in atherosclerosis}

18. Perisic L, Aldi S, Sun Y, et al. Gene expression signatures, pathways and networks in carotid atherosclerosis. J Intern Med. 2016;279:293-308.

19. Redgrave TG, Roberts DC and West CE. Separation of plasma lipoproteins by density-gradient ultracentrifugation. Anal Biochem. 1975;65:42-9.

20. Aper SJ, van Spreeuwel AC, van Turnhout MC, et al. Colorful protein-based fluorescent probes for collagen imaging. PLoS One. 2014;9:e114983.

21. Bray NL, Pimentel $H$, Melsted $P$, et al. Near-optimal probabilistic RNA-seq quantification. Nat Biotechnol. 2016;34:525-7.

22. Ritchie ME, Phipson B, Wu D, et al. limma powers differential expression analyses for RNA-sequencing and microarray studies. Nucleic Acids Res. 2015;43:e47.

23. Garcia-Alonso L, Holland $\mathrm{CH}$, Ibrahim MM, et al. Benchmark and integration of resources for the estimation of human transcription factor activities. Genome Res. 2019;29:1363-1375.

24. Liberzon A, Subramanian A, Pinchback R, et al. Molecular signatures database (MSigDB) 3.0. Bioinformatics. 2011;27:1739-40.

25. Naba A, Clauser KR, Ding $H$, et al. The extracellular matrix: Tools and insights for the "omics" era. Matrix Biol. 2016;49:10-24.

26. Stuart T, Butler A, Hoffman P, et al. Comprehensive Integration of Single-Cell Data. Cell. 2019;177:1888-1902 e21.

27. Holland $\mathrm{CH}$, Szalai B and Saez-Rodriguez J. Transfer of regulatory knowledge from human to mouse for functional genomics analysis. Biochim Biophys Acta Gene Regul Mech. 2020;1863:194431.

28. Zernecke A, Winkels $\mathrm{H}$, Cochain $\mathrm{C}$, et al. Meta-Analysis of Leukocyte Diversity in Atherosclerotic Mouse Aortas. Circ Res. 2020;127:402-426.

29. Holland $\mathrm{CH}$, Tanevski J, Perales-Paton J, et al. Robustness and applicability of transcription factor and pathway analysis tools on single-cell RNA-seq data. Genome Biol. 2020;21:36.

30. Takeda K, Cowan A and Fong GH. Essential role for prolyl hydroxylase domain protein 2 in oxygen homeostasis of the adult vascular system. Circulation. 2007;116:774-81. 31. Evrard SM, Lecce L, Michelis KC, et al. Endothelial to mesenchymal transition is common in atherosclerotic lesions and is associated with plaque instability. Nat Commun. 2016;7:11853.

32. Kramann R, Goettsch C, Wongboonsin J, et al. Adventitial MSC-like Cells Are Progenitors of Vascular Smooth Muscle Cells and Drive Vascular Calcification in Chronic Kidney Disease. Cell Stem Cell. 2016;19:628-642.

33. Wirka RC, Wagh D, Paik DT, et al. Atheroprotective roles of smooth muscle cell phenotypic modulation and the TCF21 disease gene as revealed by single-cell analysis. Nat Med. 2019.

34. Browaeys R, Saelens $\mathrm{W}$ and Saeys $\mathrm{Y}$. NicheNet: modeling intercellular communication by linking ligands to target genes. Nat Methods. 2020;17:159-162.

35. Wolak T. Osteopontin - a multi-modal marker and mediator in atherosclerotic vascular disease. Atherosclerosis. 2014;236:327-37.

36. Lok ZSY and Lyle AN. Osteopontin in Vascular Disease. Arterioscler Thromb Vasc Biol. 2019;39:613-622. 
37. Kramerova I, Kumagai-Cresse C, Ermolova N, et al. Spp1 (osteopontin) promotes TGFbeta processing in fibroblasts of dystrophin deficient muscles through matrix metalloproteinases. Hum Mol Genet. 2019.

38. Strom A, Franzen A, Wangnerud C, et al. Altered vascular remodeling in osteopontin-deficient atherosclerotic mice. J Vasc Res. 2004;41:314-22.

39. Guentsch A, Beneke A, Swain L, et al. PHD2 Is a Regulator for Glycolytic Reprogramming in Macrophages. Mol Cell Biol. 2017;37.

40. Seimon T and Tabas I. Mechanisms and consequences of macrophage apoptosis in atherosclerosis. J Lipid Res. 2009;50 Suppl:S382-7.

41. Winning S, Splettstoesser F, Fandrey J, et al. Acute hypoxia induces HIFindependent monocyte adhesion to endothelial cells through increased intercellular adhesion molecule-1 expression: the role of hypoxic inhibition of prolyl hydroxylase activity for the induction of NF-kappa B. J Immunol. 2010;185:1786-93.

42. Beneke A, Guentsch A, Hillemann A, et al. Loss of PHD3 in myeloid cells dampens the inflammatory response and fibrosis after hind-limb ischemia. Cell Death Dis. 2017;8:e2976.

43. Simoes FC, Cahill TJ, Kenyon A, et al. Macrophages directly contribute collagen to scar formation during zebrafish heart regeneration and mouse heart repair. Nat Commun. 2020;11:600.

44. Haider N, Bosca L, Zandbergen HR, et al. Transition of Macrophages to FibroblastLike Cells in Healing Myocardial Infarction. J Am Coll Cardiol. 2019;74:3124-3135.

45. Shao JS, Sierra OL, Cohen R, et al. Vascular calcification and aortic fibrosis: a bifunctional role for osteopontin in diabetic arteriosclerosis. Arterioscler Thromb Vasc Biol. 2011;31:1821-33.

46. lida T, Wagatsuma K, Hirayama D, et al. Is Osteopontin a Friend or Foe of Cell Apoptosis in Inflammatory Gastrointestinal and Liver Diseases? Int J Mol Sci. 2017;19.

47. Wang JB, Zhang Z, Li JN, et al. SPP1 promotes Schwann cell proliferation and survival through PKCalpha by binding with CD44 and alphavbeta3 after peripheral nerve injury. Cell Biosci. 2020;10:98.

48. Ramachandran P, Dobie R, Wilson-Kanamori JR, et al. Resolving the fibrotic niche of human liver cirrhosis at single-cell level. Nature. 2019;575:512-518.

49. Lee JW, Ko J, Ju C, et al. Hypoxia signaling in human diseases and therapeutic targets. Exp Mol Med. 2019;51:1-13.

50. Botusan IR, Sunkari VG, Savu O, et al. Stabilization of HIF-1alpha is critical to improve wound healing in diabetic mice. Proc Natl Acad Sci U S A. 2008;105:19426-31.

51. Nakano D, Hayashi T, Tazawa N, et al. Chronic hypoxia accelerates the progression of atherosclerosis in apolipoprotein E-knockout mice. Hypertens Res. 2005;28:837-45.

52. Chaudhari SM, Sluimer JC, Koch M, et al. Deficiency of HIF1alpha in AntigenPresenting Cells Aggravates Atherosclerosis and Type 1 T-Helper Cell Responses in Mice. Arterioscler Thromb Vasc Biol. 2015;35:2316-25.

53. Walmsley SR, Print C, Farahi N, et al. Hypoxia-induced neutrophil survival is mediated by HIF-1alpha-dependent NF-kappaB activity. J Exp Med. 2005;201:105-15.

54. Fong CC, Zhang Q, Shi YF, et al. Effect of hypoxia on RAW264.7 macrophages apoptosis and signaling. Toxicology. 2007;235:52-61. 


\section{5 | Myeloid PHD deficiency in atherosclerosis}

55. Holdstock L, Meadowcroft AM, Maier R, et al. Four-Week Studies of Oral HypoxiaInducible Factor-Prolyl Hydroxylase Inhibitor GSK1278863 for Treatment of Anemia. J Am Soc Nephrol. 2016;27:1234-44.

56. Provenzano $\mathrm{R}$, Besarab A, Sun $\mathrm{CH}$, et al. Oral Hypoxia-Inducible Factor Prolyl Hydroxylase Inhibitor Roxadustat (FG-4592) for the Treatment of Anemia in Patients with CKD. Clin J Am Soc Nephrol. 2016;11:982-91.

57. Chen N, Hao C, Peng X, et al. Roxadustat for Anemia in Patients with Kidney Disease Not Receiving Dialysis. N Engl J Med. 2019;381:1001-1010.

58. Chen N, Hao C, Liu BC, et al. Roxadustat Treatment for Anemia in Patients Undergoing Long-Term Dialysis. N Engl J Med. 2019;381:1011-1022.

59. Newman AA, Baylis RA, Hess DL, et al. Irradiation abolishes smooth muscle investment into vascular lesions in specific vascular beds. JCI Insight. 2018;3. 


\section{Supplemental data}
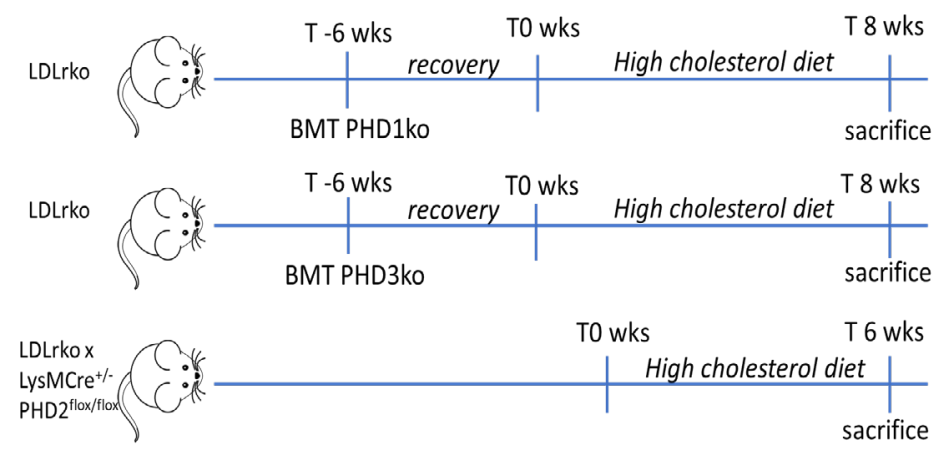

Supplemental Figure S1. Overview of mouse models used for myeloid PHD1,2 and 3 (c)KO 
A

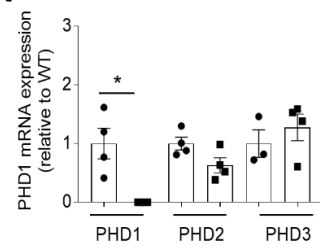

B

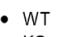

- WT
KO

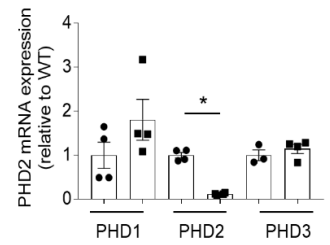

C

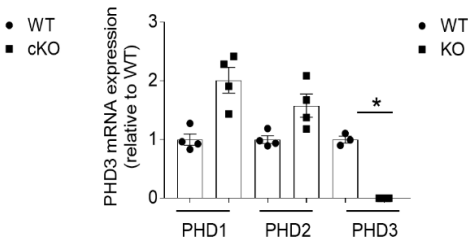

D
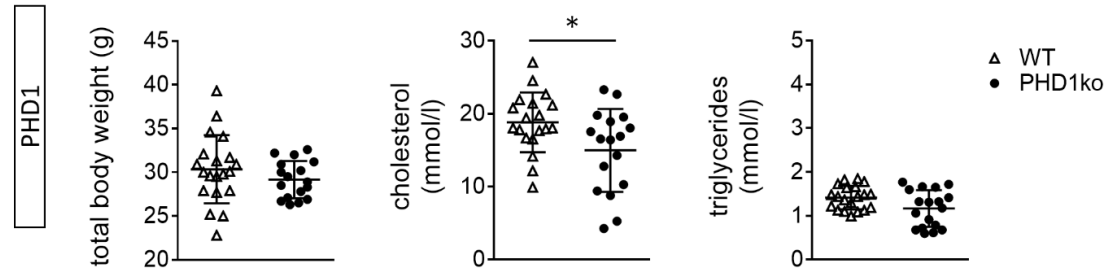

$\mathbf{E}$
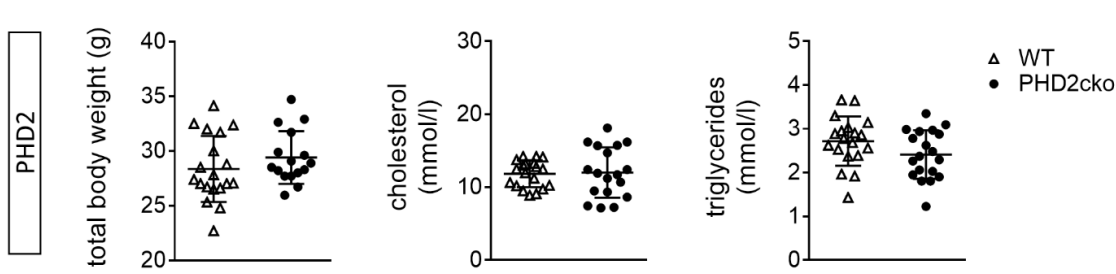

$\mathbf{F}$
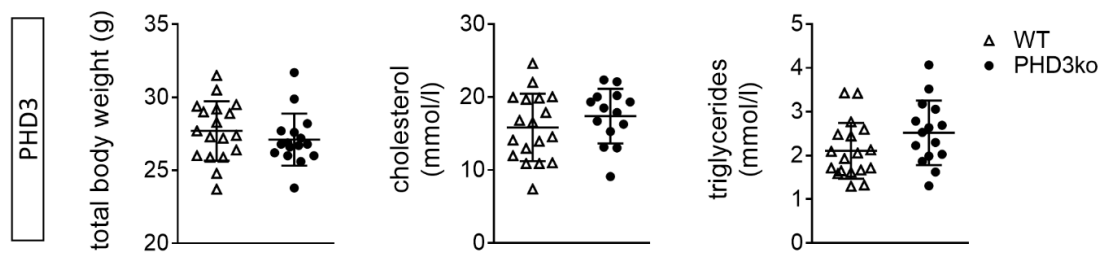

Supplemental Figure S2. Knockdown confirmation and circulating lipids in PHD1KO, PHD2cKO and PHD3KO mice (A) Expression of PHD1, (B) PHD2, and (C) PHD3 mRNA in PHD1KO, PHD2cKO and PHD3KO BMDM compared to respective controls. (D) Total body weight, circulating cholesterol and triglycerides of PHD1KO, (E) PHD2cKO and (F) PHD3KO mice and respective controls ( $n=16-20$ per group). Statistical analyses were performed using a two-way ANOVA, with Bonferroni post-hoc test $(A-C)$ or a student t-test (D-F). All results show mean \pm SEM. ${ }^{*} \mathrm{P}<0.05$. 
A
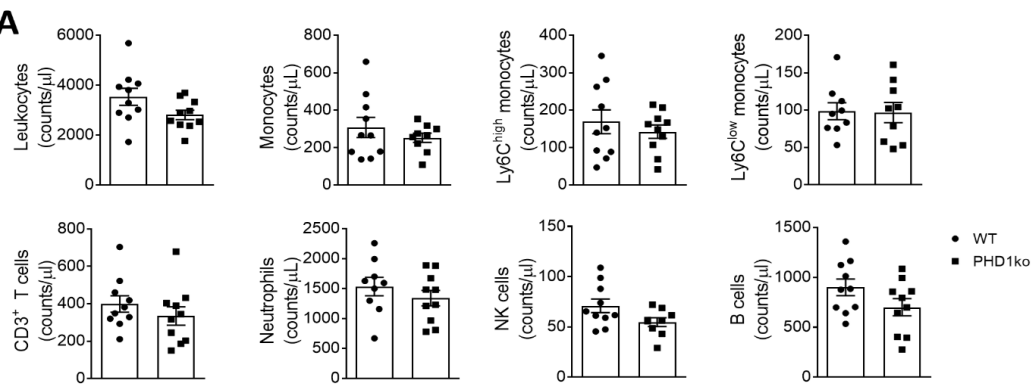

B
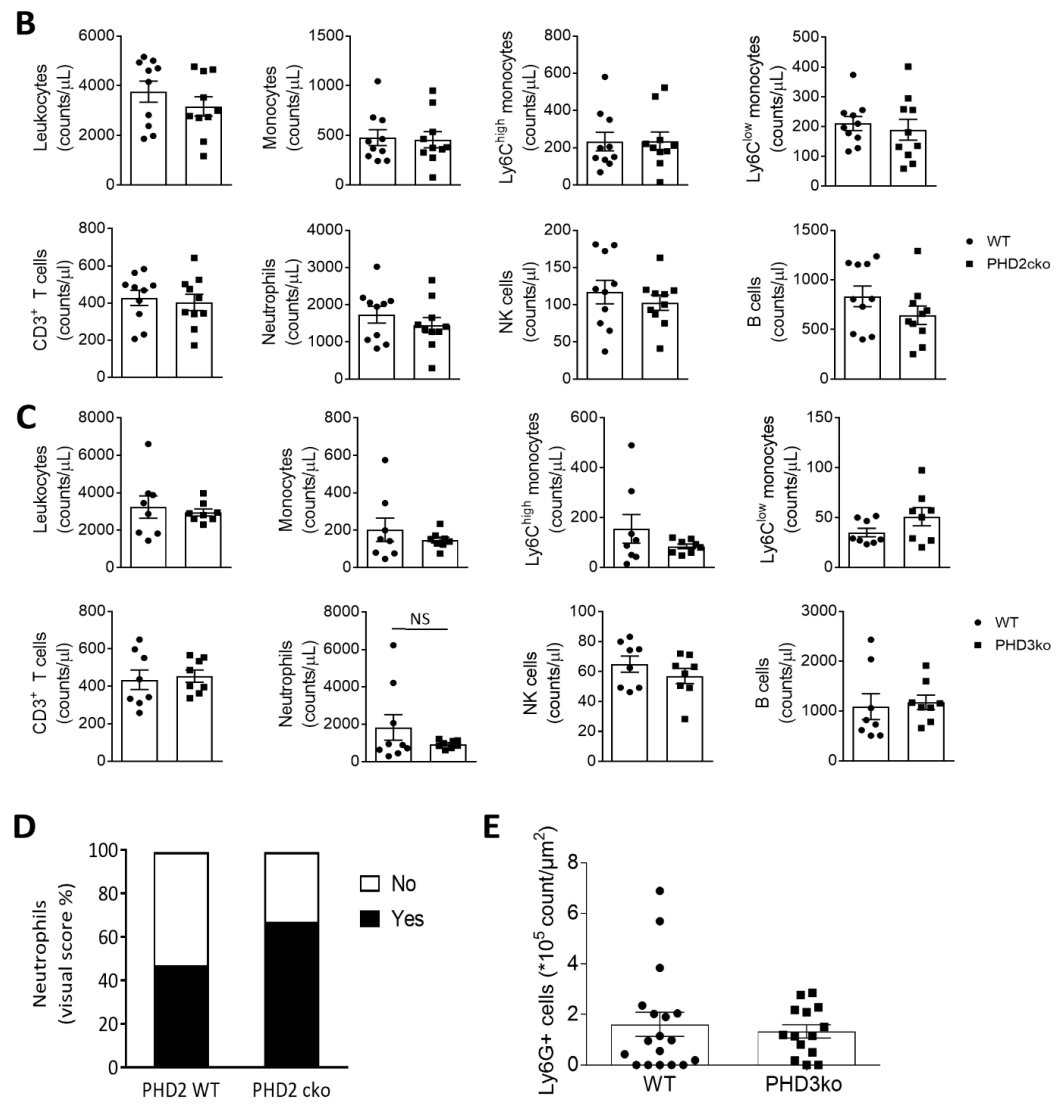

Supplemental Figure S3. No change in circulating leukocyte subsets in myeloid PHD knock-out mice and respective controls (A) Circulating leukocyte subsets of PHD1KO, (B) PHD2cKO, and (C) PHD3KO mice (black bars) and respective controls (white bars, $\mathrm{n}=20$ per group for PHD1KO and PHD2cKO, $\mathrm{n}=16-18$ per group for PHD3KO). (D) Neutrophils presence scored as present (Yes) vs. not present (No) by animal pathologist MG. (E) Quantification of Ly6G positive neutrophils in PHD3ko and corresponding WT plaques, $\mathrm{N}=18$ per group. Statistical analyses were performed using a student $\mathrm{t}$ test $(\mathbf{A}-\mathbf{C}, \mathbf{E})$. All results show mean \pm SEM. 
5 | Myeloid PHD deficiency in atherosclerosis

A

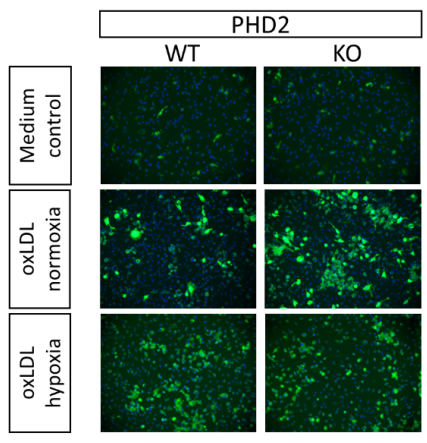

C

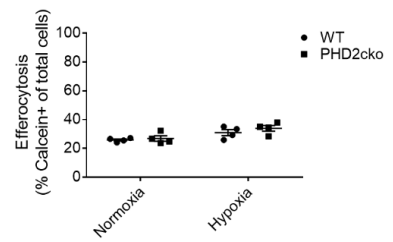

B
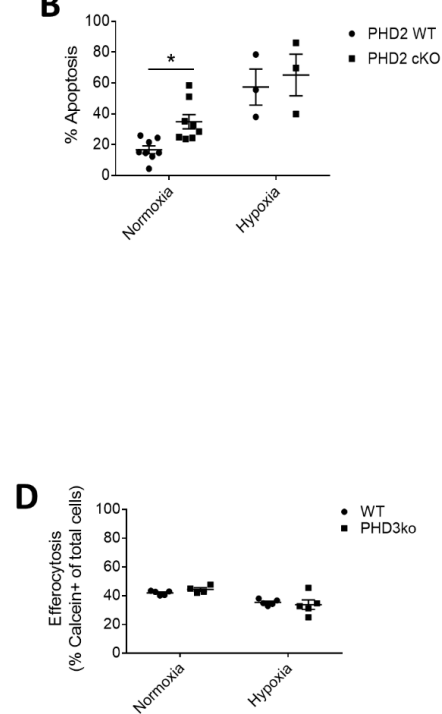

Supplemental Figure S4. Unchanged efferocytosis in PHD2cKO and PHD3KO BMDMs (A) Representative images and (B) quantifications of in vitro WT and PHD2cKO BMDM apoptosis induced by oxLDL in normoxic and hypoxic conditions. (C-D) In vitro BMDM efferocytosis under normoxic and hypoxic conditions. Expressed as the percentage of Calcein positive BMDMs. Statistical analyses were performed using a two-way ANOVA, with Bonferroni post-hoc test (B-D).All results show mean \pm SEM. 
A

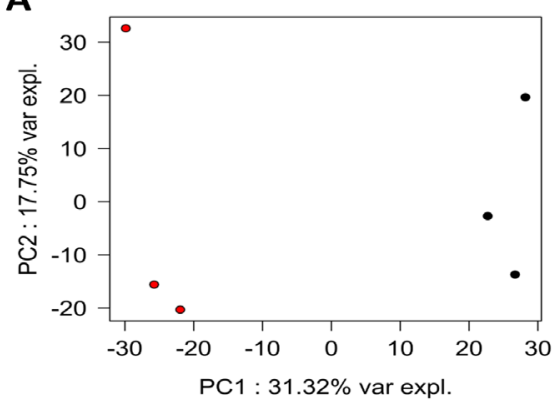

C

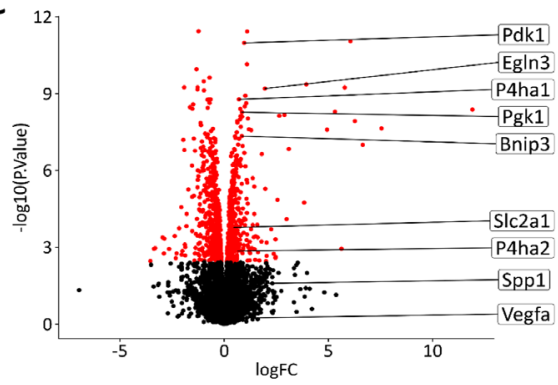

significant - FDR<0.05 - n.s.

$\mathbf{E}$
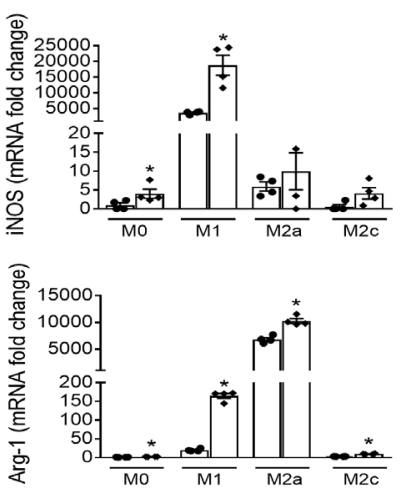

B

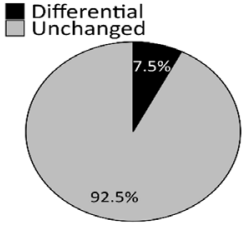

Total 14285 genes

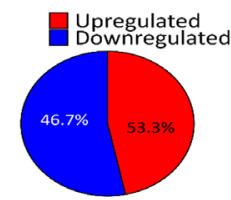

$7.5 \%$ of the total genes

D
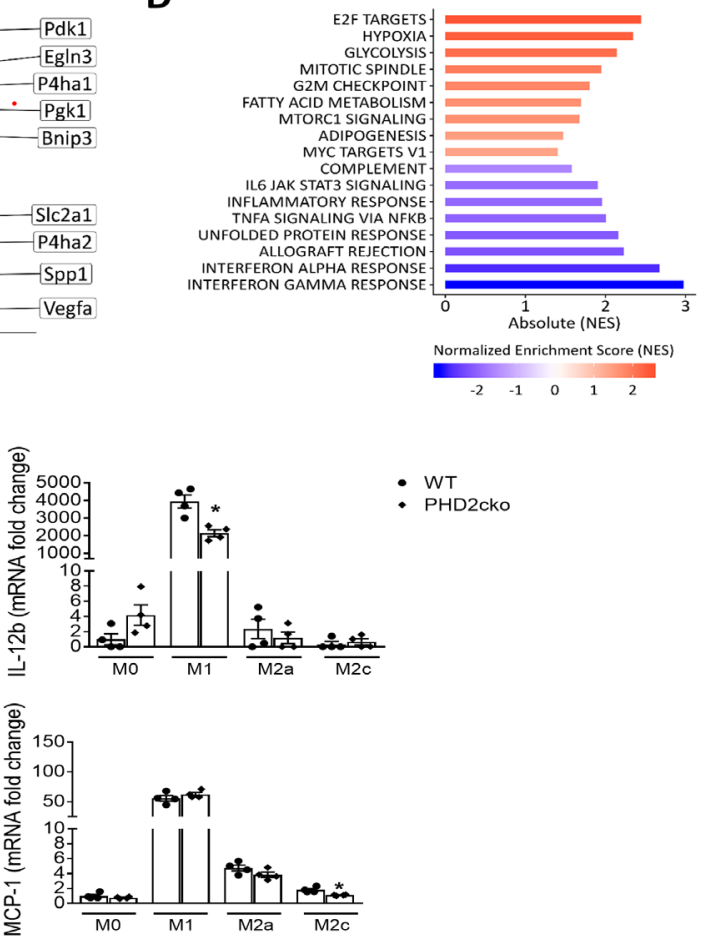

Supplemental Figure S5. Transcriptomic analysis of PHD2cKO and WT BMDMs in vitro (A) Principal component analysis depicting separation of samples depending on genotype. Black: WT. Red: PHD2CKO. (B) Pie chart showing number of differentially up and down regulated genes in BMDM. (C) Volcano plot showing significant differentially expressed genes between WT and PHD2cKO BMDMs in red, with genes relevant for hypoxia signaling and apoptosis marked. Triplicate samples for RNAseq in each group. (D) Barplot showing the Normalized Enrichment Scores (NES) for the statistically significant hallmark gene sets enriched in BMDM PHD2cKO as compared to WT from the pre-ranked GSEA. (E) Expression of macrophage polarization genes in PHD2cko and corresponding WT bone marrow derived macrophages measured by qRT-PCR. All polarization stimuli were given for 24 hours. $\mathrm{M} 1$ received IFNY $(100 \mathrm{u} / \mathrm{ml})$ and LPS $(10 \mathrm{ng} / \mathrm{ml}), \mathrm{M} 2 \mathrm{~A}$ received IL-4 $(20 \mathrm{ng} / \mathrm{ml})$ and $\mathrm{M} 2 \mathrm{C}$ received IL-10 $(10 \mathrm{ng} / \mathrm{ml})$. Statistical analyses were performed using a two-way ANOVA, with Bonferroni post-hoc test (E). All results show mean $\pm S E M$. ${ }^{*} P<0.05$. 


\section{5 | Myeloid PHD deficiency in atherosclerosis}
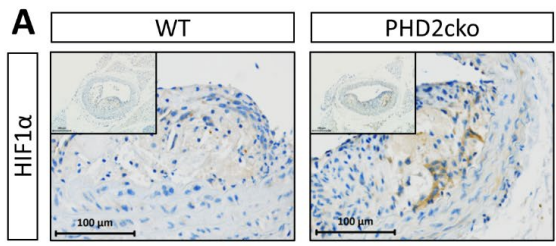

B

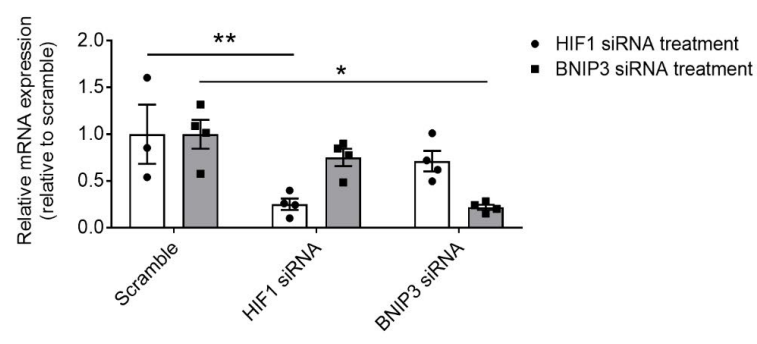

C
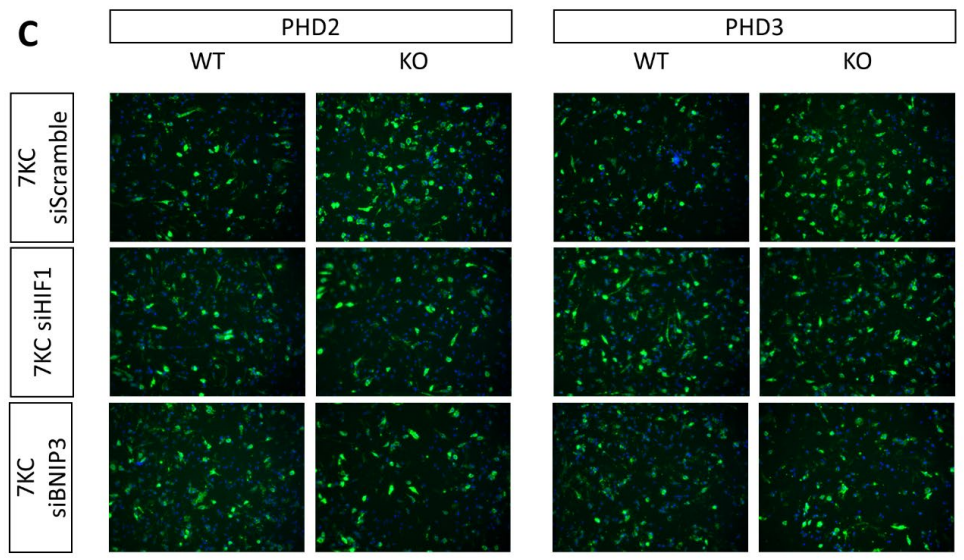

Supplemental Figure S6. HIF1 $\alpha$ and BNIP3 expression and silencing (A) Representative microphotograph of HIF1 stained plaque in murine WT and PHD2cKO brachiocephalic artery. Scalebar $100 \mu \mathrm{m}$. (B) Expression of HIF1 $\alpha$ (white bars) and BNIP3 mRNA (black bars) upon silencing with respective siRNA on y-axis compared to scramble siRNA. (C) Representative microphotographs of in vitro apoptosis measured by ANX5-FP488 induced by 7KC in control, PHD2cKO and PHD3KO after transfection with scramble (SCR), HIF1 $\alpha$, and BNIP3 siRNA. Statistical analyses were performed using a two-way ANOVA, with Bonferroni post-hoc test (B). All results show mean \pm SEM. 

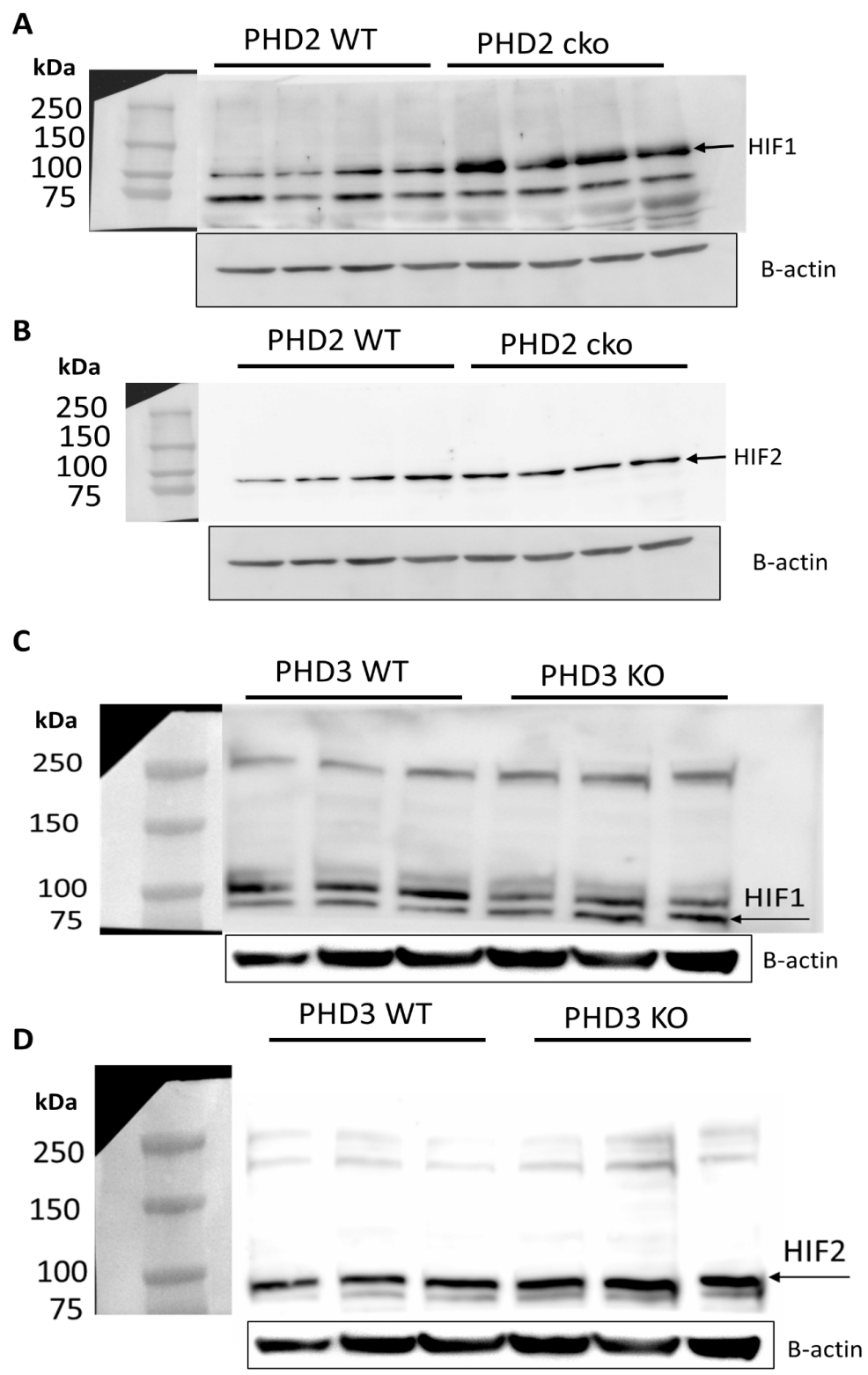

Supplementary Figure S7. Full western blots for figures 3F-G. PHD2 WT vs cko protein expression of HIF1 (A) and HIF2 (B). PHD3 WT vs. ko protein expression of HIF1 (C) and HIF2 (D). All with corresponding b-actin control. Blots were cut at 75kDa. 

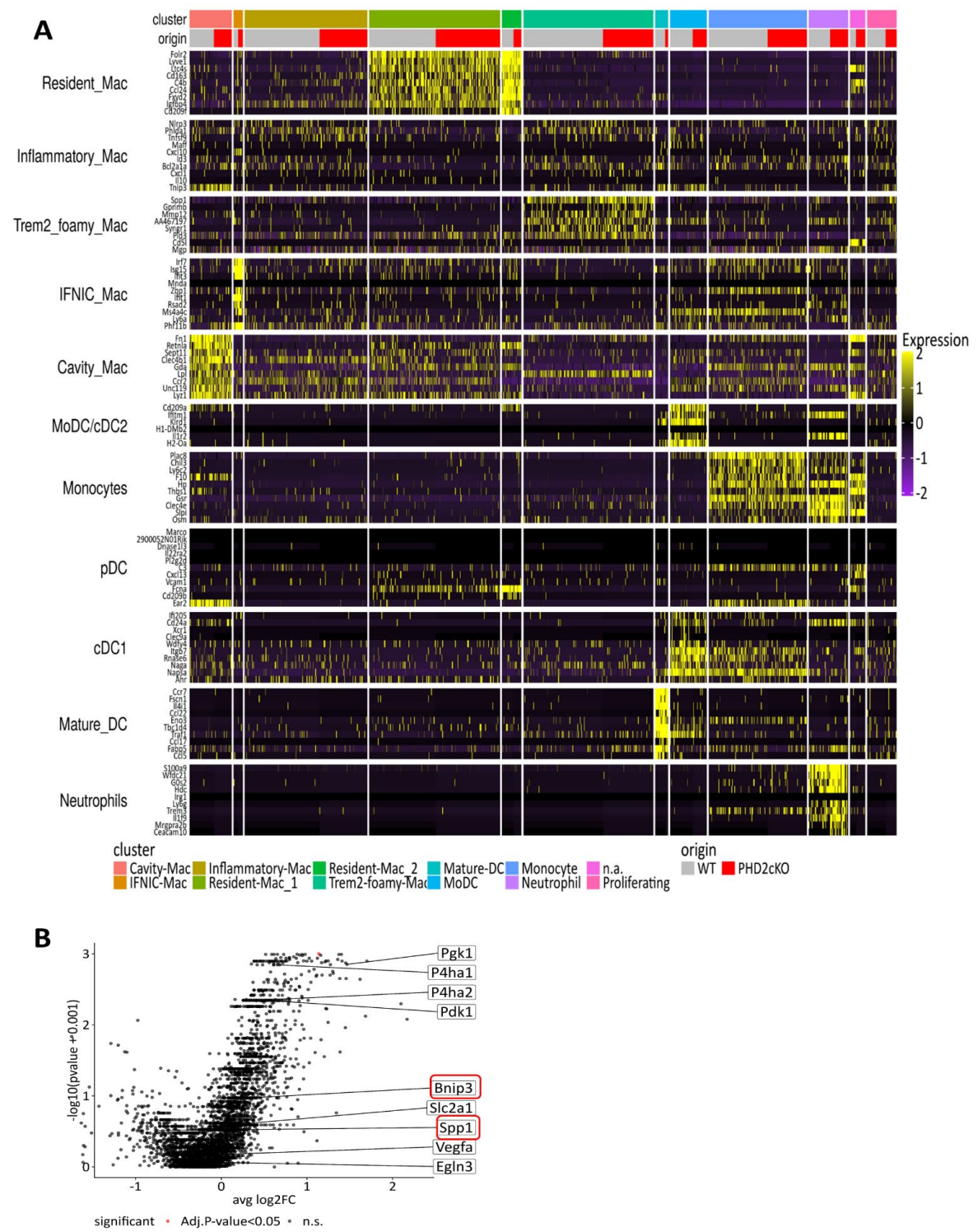

Supplementary Figure S8. Cell annotation of myeloid leukocytes in single cell sequencing dataset from PHD2 WT and cko plaques. (A) Heatmap depicting cell type annotation based on the top 10 markers reported from Zernecke et al. ${ }^{28}$. Rows are genes. Columns are cells. Columns were grouped by the outcome from unsupervised clustering (12 clusters). Gene-level expression was scaled across cells. (B) Volcano plot depicting differentially expressed genes in high PHD2cko signature neutrophils ( $n=7$ cells) vs. low PHD2cko signature neutrophils ( $n=30$ cells). 
A
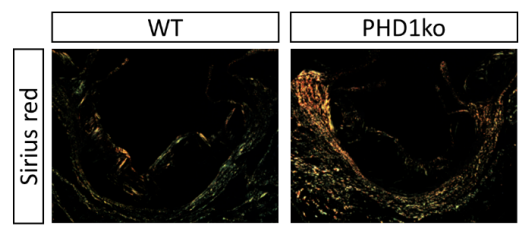

C

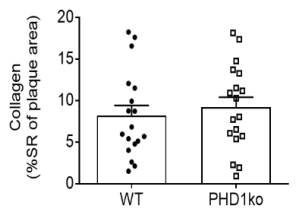

B
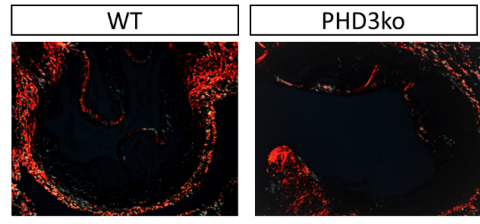

D

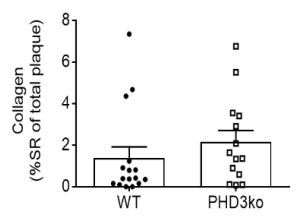

Supplemental Figure S9. Myeloid PHD1KO and PHD3KO did not affect plaque collagen (A) Representative microphotographs of Sirius red stained aortic root sections of PHD1KO, and (B) PHD3KO mice and respective controls. (C) Quantification of Sirius red positive area of PHD1KO and (D) PHD3KO mice and their controls in 16-20 mice per group. Statistical analyses were performed using a student t-test $(\mathbf{C}-\mathbf{D})$. All results show mean \pm SEM. 
A
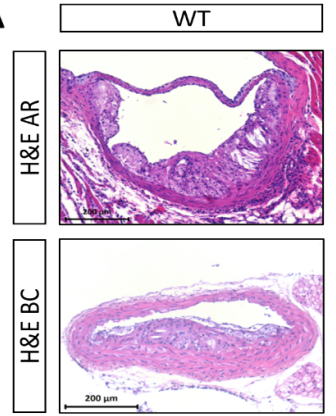

C
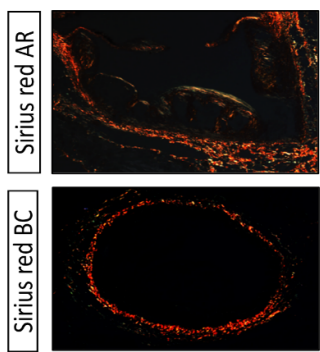

E
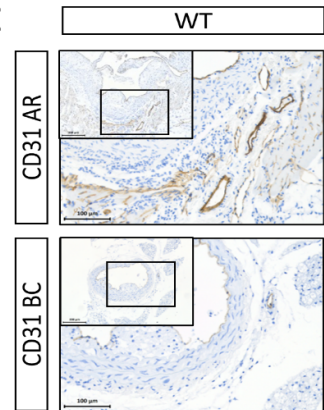

G

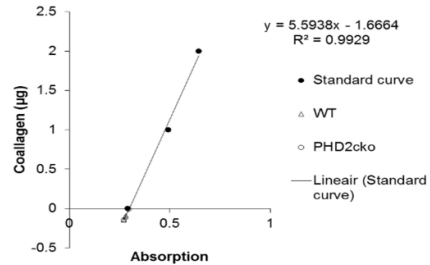

B
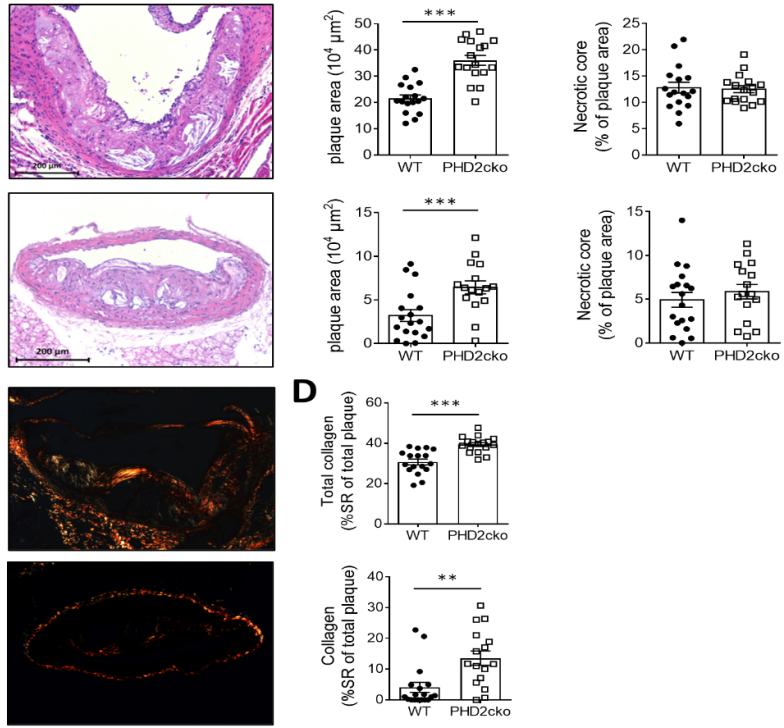

$\mathbf{F}$
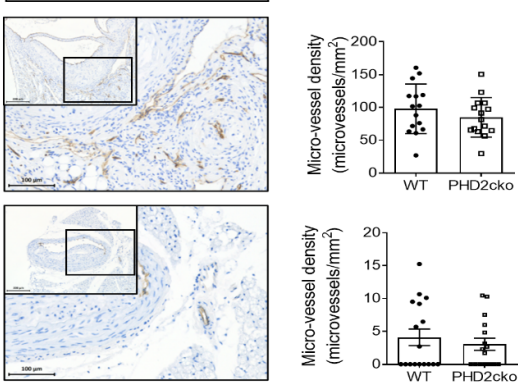

Supplemental Figure S10. Increased plaque size in PHD2cKO mice is preserved in 12 weeks HCD diet fed mice, and two vascular beds (A) Representative microphotographs of aortic root lesions stained with HE in PHD2cKO and WT control mice fed a 12-week high-cholesterol diet. (B) Quantification of plaque size and necrotic content. (C) Sirius red stained aortic root and brachiocephalic (BC) artery sections of WT and PHD2cKO mice fed a 12-week HCD. (E) Representative microphotographs of CD31 stained aortic root and brachiocephalic sections of PHD2cKO and WT control mice. (F) Quantification of micro-vessel density in adventitia of aortic root and brachiocephalic artery. All groups contain 1620 mice. Statistical analyses were performed using a student t-test (B, D, F). All results show mean \pm SEM. ${ }^{* * *} \mathrm{P}<0.001$. Scale bar HE $200 \mu \mathrm{m}, \mathrm{CD} 31100 \mu \mathrm{m}$. (G) Absorption and collagen of BMDM supernatant and standard curve samples. 

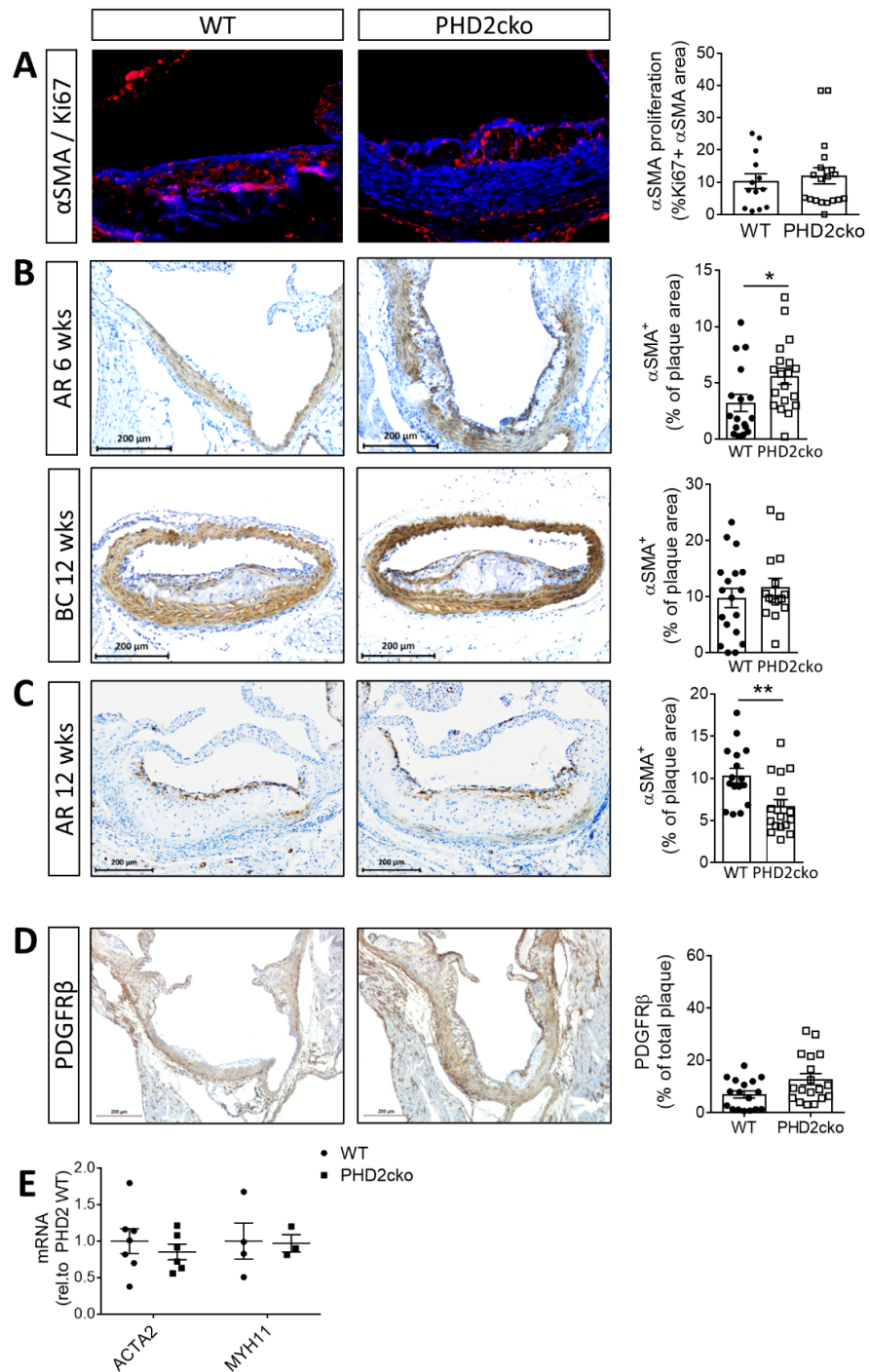

Supplemental Figure S11. Plaque content of $\alpha$ SMA in 6 and 12 week HCD diet fed PHD2cKO mice, and two vascular beds (A) Pseudo-fluorescent image of $\alpha \mathrm{SMA} / \mathrm{Ki67}$ (red/blue, respectively) staining of mesenchymal (MC) proliferation in the aortic root plaque. Magnification 400x. (B) Representative microphotographs and quantification of plaque aSMA content in PHD2cKO and WT control mice of aortic roots fed a 6-week high-cholesterol diet, and (C). In brachiocephalic and aortic root of mice fed a 12-week high-cholesterol diet. All groups contain 16-20 mice. (D) Representative microphotographs and quantification of PDGFR (brown precipitate, DAB) in aortic root of mice fed a six-week highcholesterol diet. Scalebar $100 \mu \mathrm{m}$. (E) mRNA expression of myogenic genes in 3T3 fibroblasts incubated in PHD2cKO BMDM conditioned medium for 72 hours. Statistical analyses were performed using a student t-test $(A-D)$ or two-way ANOVA with Bonferroni post-hoc test $(E)$. All results show mean \pm SEM. ${ }^{*} \mathrm{P}<0.05 * * \mathrm{P}<0.01$. Scale bar $200 \mu \mathrm{m}$. 


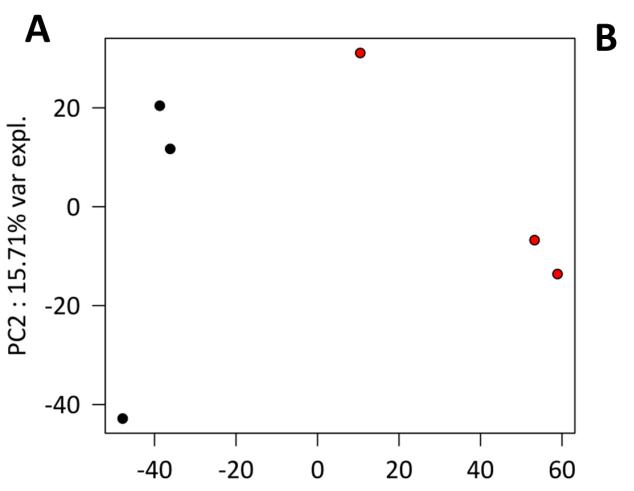

B

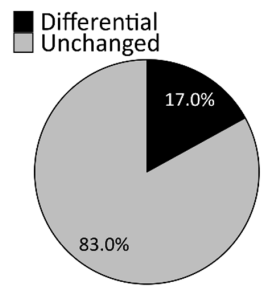

Total 14285 genes

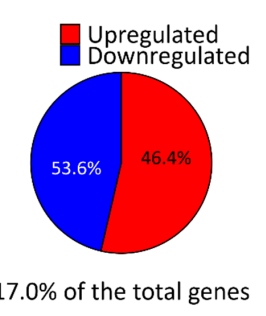

PC1 : 50.42\% var expl.
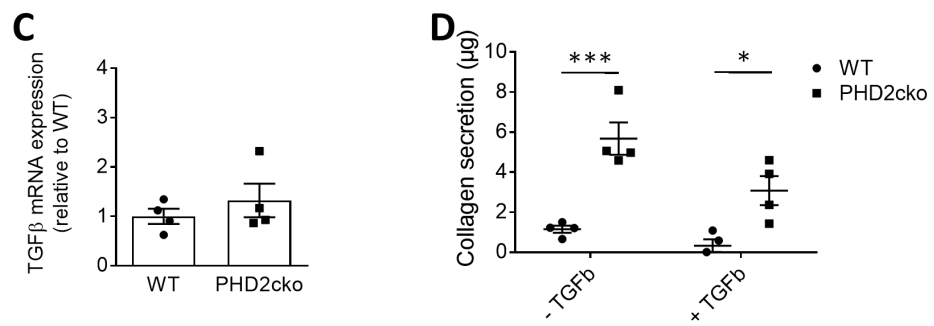

Supplemental Figure S12. Transcriptomic analysis of fibroblasts incubated in medium conditioned by PHD2cKO and WT BMDM and in vitro stimulation fibroblasts with TGF- $\boldsymbol{\beta}$ (A)Principal component analysis depicting separation of samples depending on exposure. Black: WT-conditioned medium, Red: PHD2cKO-conditioned medium. (B) Pie chart showing number of up and down regulated genes in fibroblasts. Triplicate samples for RNAseq in each group. (C) In vitro mRNA expression of TGFB1 by WT and PHDCKO BMDM was similar. (D) Addition of TGFB to fibroblasts did not increase collagen secretion of fibroblasts incubated with WT-conditioned medium to the same level as PHD2cKOcontioned medium. Statistical analyses were performed using a student t-test (C) or two-way ANOVA with Bonferroni post-hoc test (D). All results show mean \pm SEM. ${ }^{*} \mathrm{P}<0.05 * * * \mathrm{P}<0.001$. 


\section{Myeloid PHD deficiency in atherosclerosis | 5}

Supplemental Table S1. Primer sequences used for qRT-PCR

\begin{tabular}{|c|c|c|}
\hline Gene & Forward primer (5'-3') & Reverse primer (5'-3') \\
\hline $18 S$ & GTAACCCGTTGAACCCCATT & CCATCCAATCGGTAGTAGCG \\
\hline Cyclo & CAAATGCTGGACCAAACACAA & AGTTAGCATGCCAGAGTCTCGTT \\
\hline PHD1 & CATCAATGGGCGCACCA & GATTGTCAACATGCCTCACGTAC \\
\hline PHD2 & TAAACGGCCGAACGAAAGC & GGGTTATCAACGTGACGGACA \\
\hline PHD3 & CTATGTCAAGGAGCGGTCCAA & GTCCACATGGCGAACATAACC \\
\hline BNIP3 & CCTGTCGCAGTTGGGTTC & GAAGTGCAGTTCTACCCAGGAG \\
\hline HIF1 $\alpha$ & TGCTCATCAGTTGCCACTTC & CCATCTGTGCCTTCATCTCA \\
\hline HIF2 $\alpha$ & ACCCCGAGGAGCTACTTGGA & GATACCACCTGCCCCTTGGT \\
\hline TGF $\beta$ & GCCCTTCCTGCTCCTCATG & CCGCACACAGCAGTTCTTCTC \\
\hline ACTA2 & GACTACTGCCGAGCGTGAG & ATAGGTGGTTTCGTGGATGC \\
\hline MYH11 & TGGACACCATGTCAGGGAAA & ATGGACACAAGTGCTAAGCAGTCT \\
\hline
\end{tabular}


5 | Myeloid PHD deficiency in atherosclerosis

Supplemental Table S2. Single Cell Sequencing report

\begin{tabular}{|c|c|c|c|c|c|c|c|}
\hline & Sequencing & \multicolumn{2}{|c|}{ Mapping* } & \multicolumn{3}{|c|}{ Cells } & Sequencing \\
\hline Sample & $\begin{array}{c}\text { Total raw } \\
\text { reads }\end{array}$ & Genome & Exons & Cell cnt & $\begin{array}{c}\text { Reads/c } \\
\text { ell\# }\end{array}$ & $\begin{array}{c}\text { Genes/c } \\
\text { ellt }\end{array}$ & Sat. curve \\
\hline WT & $148 \mathrm{M}$ & $89.90 \%$ & $73.60 \%$ & 2,087 & 70,848 & 1,978 & $84 \%$ (OK) \\
\hline PHD2cKO & $85 \mathrm{M}$ & $87.60 \%$ & $71.20 \%$ & 1,444 & 58,954 & 1,595 & $84 \%$ (OK) \\
\hline
\end{tabular}

*, percentage of reads mapped confidently; cnt, count; \#, mean; † median; Sat. saturation 


\section{Myeloid PHD deficiency in atherosclerosis | 5}

Supplemental Table 3. Pearson correlation of PHD3 mRNA expression with CD68, HIF1 and HIF2 mRNA expression in plaques of BiKE cohort subjects $(n=127)$

\begin{tabular}{|c|c|c|c|c|c|c|c|}
\hline \multicolumn{3}{|c|}{ PHD1 } & Correlation & p value & Correlation & p value \\
\hline & Correlation & p-value & & & & & \\
\hline CD68 & -0.17 & 0.064 & & 0.42 & $<0.001$ & 0.64 & $<0.001$ \\
\hline HIF1 & -0.28 & 0.002 & 0.44 & $<0.001$ & 0.43 & $<0.001$ \\
\hline HIF2 & 0.19 & 0.032 & -0.18 & 0.047 & -0.28 & 0.002 \\
\hline
\end{tabular}


5 | Myeloid PHD deficiency in atherosclerosis

Supplemental Table 4. PROGENy pathway analysis of differentially expressed genes between WT and PDH2cKO BMDM

\begin{tabular}{|c|c|c|}
\hline & Activity & P-value \\
\hline HYPOXIA & 14.85 & $<1.0 * 10^{-4}$ \\
\hline MAPK & 1.84 & $6.6 * 10^{-2}$ \\
\hline TGFB & 0.82 & $4.1 * 10^{-1}$ \\
\hline EGFR & 0.72 & $4.7 * 10^{-1}$ \\
\hline P53 & -0.96 & $3.4 * 10^{-1}$ \\
\hline TRAIL & -1.27 & $2.0 * 10^{-1}$ \\
\hline ESTROGEN & -1.30 & $1.9 * 10^{-1}$ \\
\hline WNT & -1.31 & $1.9 * 10^{-1}$ \\
\hline ANDROGEN & -1.84 & $6.6 * 10^{-2}$ \\
\hline VEGF & -2.39 & $1.7 * 10^{-2}$ \\
\hline PI3K & -3.14 & $1.7 * 10^{-3}$ \\
\hline TNFA & -3.74 & $1.8 * 10^{-4}$ \\
\hline NFKB & -6.22 & $<1.0 * 10^{-4}$ \\
\hline JAK.STAT & -11.07 & $<1.0 * 10^{-4}$ \\
\hline
\end{tabular}




\section{Myeloid PHD deficiency in atherosclerosis | 5}

Supplemental Table 5. DoRothEA Transcription Factor analysis between WT and PDH2CKO BMDM

\begin{tabular}{|c|c|c|c|c|c|c|}
\hline & $\log F C$ & AveExpr & $t$ & P-Value & adj.PVal & B \\
\hline RFX5 & -0.48 & -0.94 & -6.329 & 0.0001 & 0.0051 & 2.220 \\
\hline FOXM1 & 0.30 & 1.75 & 6.186 & 0.0001 & 0.0051 & 2.024 \\
\hline HIF1A & 0.51 & 2.82 & 6.096 & 0.0001 & 0.0051 & 1.899 \\
\hline RFXANK & -0.35 & -0.84 & -6.009 & 0.0001 & 0.0051 & 1.776 \\
\hline RFXAP & -0.35 & -0.84 & -6.009 & 0.0001 & 0.0051 & 1.776 \\
\hline BCL3 & -0.21 & 4.60 & -4.845 & 0.0005 & 0.0247 & 0.033 \\
\hline IRF1 & -0.61 & -0.96 & -4.702 & 0.0007 & 0.0266 & -0.196 \\
\hline GL/2 & 0.29 & -0.78 & 4.333 & 0.0012 & 0.0426 & -0.798 \\
\hline
\end{tabular}

Only differentially active transcription factors are shown (FDR < 0.05). LogFC: estimate of the log2fold-change Transcription factor activity corresponding to the effect or contrast. AveExpr: average log2-expression. t: moderated t-statistic. P-Value: raw p-value. adj.PVal, adjusted p-value (FDR). B: log-odds that the gene is differentially expressed 


\section{5 | Myeloid PHD deficiency in atherosclerosis}

Supplemental Table 6. GSEA pathway analysis of pre-ranked matrisome pathways, differentially expressed between fibroblasts incubated in WT- or PDH2cKO-BMDM conditioned medium

\begin{tabular}{|c|c|c|c|c|c|c|}
\hline Pathway & p-val & p-adj & ES & NES & Size & LEADING EDGE \\
\hline Collagens & 0.026 & 0.043 & 0.47 & 1.55 & 31 & $\begin{array}{l}\text { COL3A1, COL5A3, COL15A1, } \\
\text { COL28A1, COL18A1, COL27A1, } \\
\text { COL5A1, COL1A1, COL5A2 }\end{array}$ \\
\hline $\begin{array}{l}\text { ECM } \\
\text { Glycoproteins }\end{array}$ & 0.029 & 0.043 & -0.33 & -1.43 & 115 & $\begin{array}{l}\text { TINAGL1, FBLN5, WISP2, CYR61, } \\
\text { SMOC2, THBS1, NOV, IGFBP4, } \\
\text { BMPER, MFGE8, MGP, NPNT, } \\
\text { LTBP1, EMILIN2, CTGF, LAMA5, } \\
\text { SMOC1, THBS2, AEBP1, SLIT2, } \\
\text { IGFBP6, PCOLCE }\end{array}$ \\
\hline ECM Regulators & 0.234 & 0.281 & -0.26 & -1.12 & 139 & $\begin{array}{l}\text { SERPINE2, ADAMTSL5, LOX, } \\
\text { CD109, MMP2, TIMP2, } \\
\text { ADAMTS1, SERPINA1A, MMP14, } \\
\text { SERPINE1, ADAMTS5, TIMP4, } \\
\text { ITIH4, SERPINB1B, SERPINB5, } \\
\text { PLOD3, MASP1, ADAMTS16, } \\
\text { HYAL2, HYAL1, PAPPA2, AGT, } \\
\text { SERPINB9, SERPINC1, SERPINB8, } \\
\text { OGFOD1, ADAM23, P4HTM, } \\
\text { SULF2, MMP11, ADAM11, } \\
\text { KAZALD1, CST3, OGFOD2 }\end{array}$ \\
\hline $\begin{array}{l}\text { ECM-affiliated } \\
\text { Proteins }\end{array}$ & 0.008 & 0.023 & 0.38 & 1.57 & 92 & $\begin{array}{l}\text { PLXNC1, SEMA7A, SDC3, } \\
\text { CLEC4A1, PLXDC1, CLEC12A, } \\
\text { CLEC5A, SEMA4D, CLEC4D, } \\
\text { CLEC7A, SEMA3C, CLEC4E, C1QA, } \\
\text { CLEC4N, OVGP1, C1QC, } \\
\text { CLEC4A2, C1QB, CLEC4A3, } \\
\text { PLXNA2, ANXA2, SEMA3A, } \\
\text { PLXND1, CLEC2H, SEMA4A, } \\
\text { FCNA, C1QTNF6, ANXA6, } \\
\text { CLEC10A, CLEC9A, SEMA4C }\end{array}$ \\
\hline
\end{tabular}


Myeloid PHD deficiency in atherosclerosis | 5

\begin{tabular}{|c|c|c|c|c|c|c|}
\hline Proteoglycans & 0.372 & 0.372 & -0.35 & -1.05 & 22 & $\begin{array}{l}\text { PODNL1, OGN, HAPLN4, ASPN, } \\
\text { BGN, BCAN }\end{array}$ \\
\hline Secreted Factors & 0.002 & 0.011 & 0.36 & 1.59 & 160 & $\begin{array}{l}\text { IGF1, ANGPTL4, CXCL14, CCL3, } \\
\text { IGF2, CX3CL1, PDGFB, TGFB1, } \\
\text { CCL2, TNF, CCL4, CCL12, CCL11, } \\
\text { IL16, CCL5, EBI3, CXCL10, IL1RN, } \\
\text { S100A1, PF4, CCL9, CCL7, CXCL2, } \\
\text { CCL6, CXCL12, TNFSF14, CCL17, } \\
\text { FSTL1, FGF13, FGF11, TNFSF13, } \\
\text { S100A8, S100A13, S100A16, } \\
\text { CLCF1, OSM }\end{array}$ \\
\hline
\end{tabular}

ECM: extracellular matrix; $p$-adj, FDR-adjusted p-value, ES, enrichment score, NES, enrichment score normalized to mean enrichment of random samples of the same size 
5 | Myeloid PHD deficiency in atherosclerosis

Supplemental Table 7. Progeny pathway analysis of differentially expressed genes of fibroblasts incubated in WT- or PDH2cKO-BMDM conditioned medium

\begin{tabular}{|c|c|c|}
\hline & Activity & P-value \\
\hline JAK.STAT & 6.60 & $4.0 * 10^{-11}$ \\
\hline TNFA & 4.78 & $1.8 * 10^{-6}$ \\
\hline NFKB & 4.37 & $1.2 * 10^{-5}$ \\
\hline MAPK & 4.28 & $1.9 * 10^{-5}$ \\
\hline Hypoxia & 3.90 & $9.7 * 10^{-5}$ \\
\hline EGFR & 1.53 & $1.3^{*} 10^{-1}$ \\
\hline PI3K & 0.97 & $3.3 * 10^{-1}$ \\
\hline WNT & 0.65 & $5.1 * 10^{-1}$ \\
\hline TRAIL & -0.03 & $9.7 * 10^{-1}$ \\
\hline Androgen & -0.27 & $7.9 * 10^{-1}$ \\
\hline Estrogen & -0.79 & $4.3 * 10^{-1}$ \\
\hline VEGF & -1.58 & $1.1 * 10^{-1}$ \\
\hline P53 & -2.26 & $2.4 * 10^{-2}$ \\
\hline TGFB & -2.91 & $3.6 * 10^{-3}$ \\
\hline
\end{tabular}


Myeloid PHD deficiency in atherosclerosis I 5 



\section{Chapter 6}

Fibroblasts in atherosclerosis: heterogeneous and plastic participants

Tillie RJHA, van Kuijk K, Sluimer JC

Curr Opin Lipidol. 2020 Oct;31(5):273-278 



\section{Abstract}

Fibroblasts are very heterogeneous and plastic cells in the vasculature. A growing interest in fibroblasts in healthy and atherosclerotic vasculature is observed, next to macrophages, endothelial cells (ECs) and smooth muscle cells (SMCs). In this review, we discuss fibroblast presence, heterogeneity, origin and plasticity in health and atherosclerosis based on latest literature. With help of single cell sequencing (SCS) techniques, we have gained more insight into presence and functions of fibroblasts in atherosclerosis. Next to SMCs, fibroblasts are extracellular matrix (ECM)-producing cells abundant in the vasculature and involved in atherogenesis. Fibroblasts encompass a heterogeneous population and SCS data reveal several fibroblast clusters in healthy and atherosclerotic tissue with varying gene expression and function. Moreover, recent findings indicate interesting similarities between adventitial stem and/or progenitor cells and fibroblasts. Also, communication with inflammatory cells opens up a new therapeutic avenue. Because of their highly plastic and heterogeneous nature, modulating fibroblast cell function and communication in the atherosclerotic vessel might be useful in battling atherosclerosis from within the plaque. 


\section{6 | Fibroblasts in atherosclerosis}

\section{Introduction}

Atherosclerosis and its clinical manifestations, e.g. myocardial infarction and stroke, are currently still the leading causes of death worldwide. ${ }^{1}$ Atherosclerosis is characterized by lipid accumulation in the sub-endothelial space, intimal inflammation, SMC migration from the media to the outside of the newly formed plaque and ultimately plaque rupture. ${ }^{2}$ Different cell types, including ECs, macrophages and SMCs, play prominent roles in this lifelong process. $^{3-5}$ However, recent evidence suggests that an additional cell type, the fibroblast, is an important player in matrix production in atherosclerosis. Traditionally, fibroblasts are thought to arise from mesenchymal stem cells (MSCs) and are thus part of the mesenchymal cell category, also including pericytes and SMCs. In arterial injury, adventitial fibroblasts differentiate into activated fibroblasts (myofibroblasts) with de novo alpha-smooth muscle actin ( $\alpha$-SMA) expression in response to pro-inflammatory cytokines, matrix remodeling and transforming growth factor beta (TGF- $\beta$ ) signaling. Myofibroblasts have been implicated in ECM production, pro-inflammatory cytokine and matrix metalloproteinase (MMP) secretion and leukocyte recruitment. ${ }^{6-8}$ However, these traditional views are being overturned by new insights and the advent of single cell sequencing (SCS), which will be discussed in this review.

In fact, the ability to acquire stem cell properties by upregulating markers such as Stem cell antigen-1 (Sca-1) enables fibroblasts to be plastic and adaptable in numerous environmental situations. ${ }^{9}, 10$ Due to this heterogeneity and plasticity, currently used markers seem insufficient in unique identification of fibroblasts and/or covering the whole fibroblast population. Here, SCS will aid to find markers unique to fibroblasts. Indeed, SCS of healthy mouse brain confirmed the traditional marker platelet-derived growth factor alpha (Pdgfro) and yielded three new markers, decorin, lumican and Mmp2. ${ }^{11}$ However, both lumican and decorin have been associated with other cell types involved in the advent of atherosclerosis. ${ }^{12,13}$ This may suggest disease- and/or organ-specificity of markers to identify fibroblasts. The lack of a one-size-fits-all marker makes investigating their role in atherosclerosis development challenging. In this review, we aim to elucidate the functional role of fibroblasts in healthy and atherosclerotic vasculature by discussing fibroblast presence, heterogeneity, origin and plasticity.

\section{Fibroblasts in healthy vasculature}

The arterial wall consists of three layers. The inner intima is composed of an EC monolayer. The middle medial layer consists of SMCs embedded in ECM. Lastly, the adventitia is the outer layer and is traditionally thought to harbor mesenchymal cells, i.e. fibroblasts, pericytes and SMCs, connective tissue, unmyelinated nerve fibers, resident leukocytes, small blood vessels with ECs surrounded by mesenchymal cells, and several progenitor cells. ${ }^{8}$ Multiple studies have shown the fibroblast's potential to extensively participate in 
organ homeostasis and repair mechanisms in response to stress. ${ }^{14-16}$ The emergence of SCS has provided researchers the opportunity to study vascular cells in more depth. This technique has improved fibroblast annotation and revealed different subsets in multiple organs. Kalluri et al. used abovementioned technique to investigate all three layers of the healthy murine aorta. ${ }^{17}$ The authors showed that SMCs comprise the largest cell population in the murine aorta ( $\sim 0 \%)$, but surprisingly, also showed that fibroblasts make up for roughly $33 \%$ of aortic cells. ${ }^{17}$ These fibroblasts consist of two subpopulations, with a phenotypic gradient rather than a rigid split between them. These fibroblasts are probably derived from the adventitia, although the authors removed perivascular fat - possibly including the adventitia. As their arterial wall location was not validated by immunohistochemistry or in situ hybridization, a possible medial location for one or both subpopulations is yet to be confirmed. Furthermore, their function, embryonic origin, cellular progeny and fate are yet unknown. Gu et al. studied the adventitia of healthy murine aorta and shed more light on their function. They uncovered four mesenchymal populations, whose differential gene expression suggests functions in ECM organization, immune regulation and bone formation. ${ }^{18}$ These data suggest fibroblast heterogeneity, already present in a healthy steady-state.

\section{Fibroblasts in atherosclerosis}

The classical dogma in atherogenesis entails migration of medial SMCs to the newly formed plaque, producing ECM components for fibrous cap formation. ${ }^{2}$ This dogma has recently been challenged, as several groups have reported the presence of fibroblast-like cells in human atherosclerotic lesions. ${ }^{12,} 19$ Also, adventitial fibroblast-like cells have been functionally implicated in plaque ECM production. ${ }^{19,20}$ Using $\mathrm{ApoE}^{-/}$mice on a Western diet superimposed with chronic kidney disease, Kramann et al. showed that a subset of adventitial MSC-like cells, expressing GLI family zinc finger 1 (Gli1), Sca1 and PDGFR $\beta$, migrated into the media and neointima. Gli1+ cells contributed to calcification by differentiation into osteoblast-like cells. ${ }^{20}$ In contrast, Evrard et al. reported decreased collagen and increased MMP expression in another subset of endothelial-derived, fibroblast-like cells expressing fibroblast activation protein (Fap) or fibroblast-specific protein 1 (Fsp1, S100a4 gene) in atherosclerosis, indicating a role in matrix degradation. ${ }^{19}$ In 2019, a key paper by Wirka et al. employed SCS to assess cellular composition in atherosclerotic plaques from human coronary artery and mouse aorta, and identified two fibroblast clusters. ${ }^{12}$ Interestingly, Gli1, Fap or Fsp1 were not among the top 100 differential genes in the two murine or human fibroblast subsets defined by Wirka, complicating the interpretation of the above reference studies and strongly suggesting heterogeneity. Together, these studies suggest that fibroblast clusters identified in healthy and diseased tissue differ in functionality, possibly due to different origin and/or differentiation fate. 


\section{6 | Fibroblasts in atherosclerosis}

\section{Fibroblast plasticity, heterogeneity and origin in atherosclerosis}

As described above, varying numbers of fibroblast clusters with corresponding differential gene sets have been identified in healthy and atherosclerotic tissue. Additionally, studies in other organs have shown that new fibroblast clusters can arise as a consequence of disease, further supporting plasticity and heterogeneity. ${ }^{15}$ Heterogeneity makes it very difficult to identify the entire fibroblast population and a resulting lack of specific markers complicates fibroblast research. Common fibroblast markers, such as FAP, FSP1 and lumican are not specifically expressed by fibroblast-like cells only, and/or are not expressed by all fibroblasts. ${ }^{21-25}$ Fibroblast heterogeneity may be a result of their various origins and enormous plasticity, all enhanced as a result of adaptation to disease. Here, we describe evidence to support that fibroblasts in atherosclerosis may also originate from SMCs and/or ECs (Figure 1). Also, we discuss adventitial stem and/or progenitor cells as a source of fibroblasts or possibly a subset of fibroblasts.

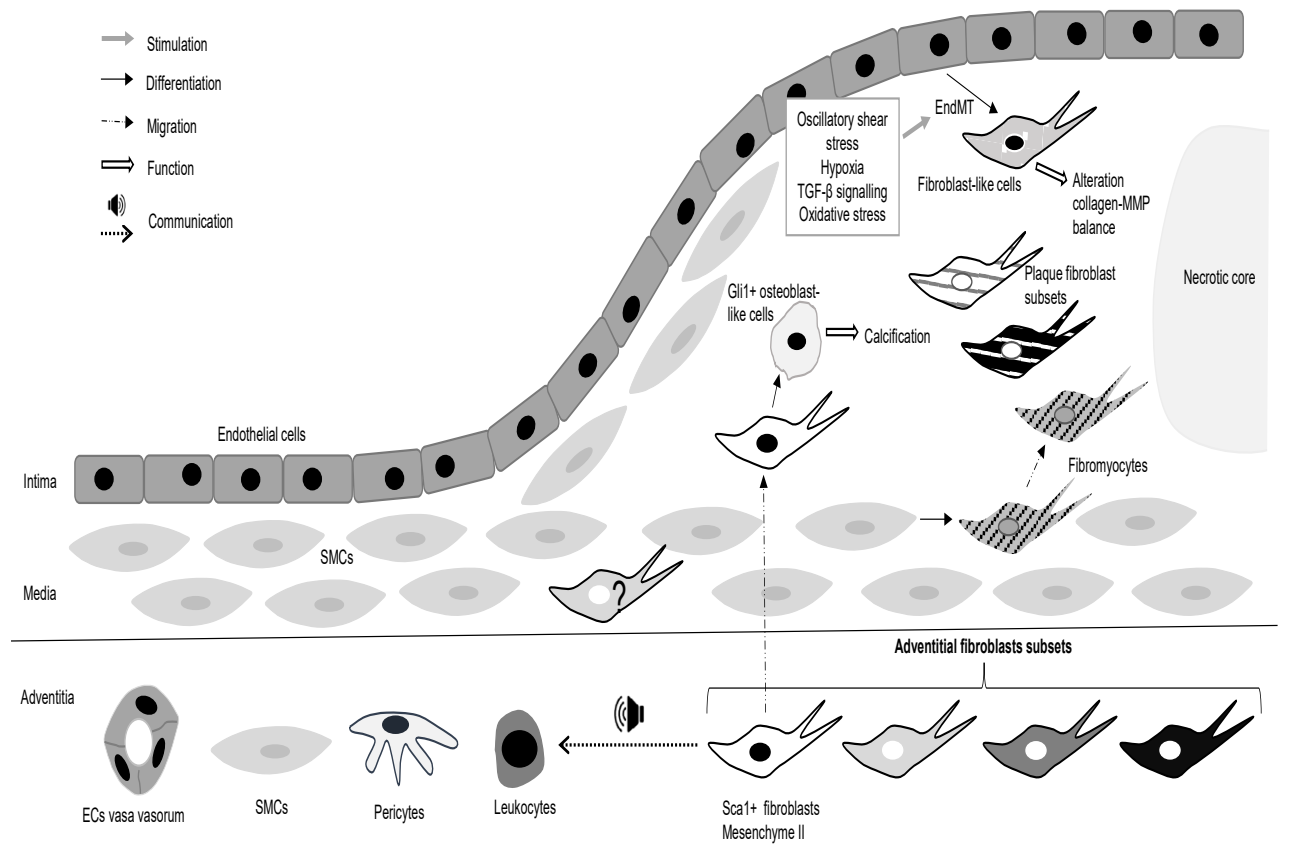

Figure 1. Presence and origin of fibroblasts in atherosclerosis and their suggested contributions. Four adventitial fibroblasts subsets have been discovered using single cell sequencing (SCS). Sca1+ fibroblasts may contribute to atherosclerosis by migrating into the neointima. Gli1+, Sca1+ adventitial stem/progenitor cells have been shown to differentiate into osteoblast-like cells and hereby contribute to plaque calcification. Two fibroblast subsets have been identified with SCS, while prior studies showed that fibroblast(-like) cells in atherosclerosis can originate from medial smooth muscle cells (SMCs), called fibromyocytes, and from endothelial cells (ECS) through endothelial-to- 
mesenchymal transition (EndMT). It is unclear whether these are similar to or distinct from the two fibroblast subsets discovered by SCS. ? indicates possible medial localization of fibroblasts.

\section{SMC origin}

Wirka et al. studied SMC differentiation and their contribution to atherosclerosis in vivo combining SCS and a fluorescent myosin heavy chain 11 (Myh11) reporter strain for SMCs on an $\mathrm{ApoE}^{-/}$background. In contrast to prevailing concepts of myofibroblast development from fibroblasts, they reported SMC differentiation into fibroblast-like, "fibromyocyte" cells upon high-fat diet (HFD). ${ }^{12}$ In addition to two fibroblast and two SMC clusters in $\mathrm{ApoE}^{-/-}$mice on chow, a Myh11+ SMC cluster appeared and expanded with HFD feeding. This modulated SMC cluster showed decreased expression of SMC differentiation markers, and a clear transcriptional shift towards genes expressed by the fibroblast clusters, later confirmed in human coronary arteries. ${ }^{12}$ Nevertheless, the cells were transcriptionally distinct from fibroblasts and displayed the Myh11-reporter. These data highlighted the benefits of fluorescent fate tracking and lead one to wonder whether these fibroblast-like cells have reached the end stage of their dedifferentiation or will dedifferentiate further into actual fibroblasts. Another question is, does the differentiation also occur the other way around? Comparison between the modulated SMC cluster and a myofibroblast population could be interesting to avoid off-target effects in future cell-specific targeting.

\section{Endothelial origin}

Another possible fibroblast source are ECs, which can undergo endothelial to mesenchymal transitioning (EndMT). A review by Kovacic et al. emphasized the functional importance of EndMT in both healthy and diseased vasculature. ${ }^{26}$ EndMT results in downregulation of endothelial-associated genes, such as cluster of differentiation (CD)31 or VE-cadherin, and upregulation of mesenchymal genes, such as $\alpha$-SMA and FAP. These cells genetically present as mesenchymal cells and can execute mesenchymal functions like ECM production. ${ }^{26}$ Evrard et al. specifically showed that fibroblasts can arise through EndMT in atherosclerosis. ${ }^{19}$ Using a tamoxifen-inducible endothelial lineage-tracking system in ApoE' I- mice, they observed one-third of plaque cells positive for Fap were endothelial-derived after 8 weeks of HFD. The population expanded to nearly $50 \%$ in advanced atherosclerotic plaques. ${ }^{19}$ They showed that EndMT is stimulated in vitro by severe hypoxia, TGF- $\beta$ signaling and oxidative stress, factors that are ubiquitous in atherosclerosis. ${ }^{19}$ Oscillatory shear stress has also been identified as EndMT inducer in atherosclerosis. ${ }^{27}$ Importantly, Evrard et al. uncovered a relationship between the extent of EndMT and an unstable plaque phenotype in humans. ${ }^{19}$ Notably, the data should be interpreted with slight caution, as the markers used to identify fibroblasts are not unique. ${ }^{22,23}$ Current SCS publications have not explicitly reported on EndMT, either because it was unstudied or possibly due to lack of sufficient cells to model transitions. However, the reported two fibroblast human subsets could include EndMT-derived cells. The top 100 differential genes do not include endothelial 


\section{6 | Fibroblasts in atherosclerosis}

markers, yet this does not exclude low marker expression. ${ }^{12}$ Hence, SCS using EC reporter strains are yet to fully confirm these findings. In addition, the functional differences between both EndMT- and SMC-derived fibroblast(-like) cells and their exact contribution to atherosclerosis remain to be elucidated.

\section{Adventitial stem and/or progenitor cells}

Fibroblasts have been suggested to originate from a pool of adventitial stem and/or progenitor cells. However, the identity of these cells is a point of discussion, as fibroblasts also have the ability to re-acquire stem cell properties by upregulating markers such as Sca1. ${ }^{9,10,20,28}$ Additionally, MSCs and fibroblasts are morphologically similar and expression of MSC surface markers, such as CD105, CD73 and CD90, has been observed on fibroblasts. Vice versa, MSC expression of common fibroblast markers, i.e. vimentin and fibroblast surface protein (FSP) has been reported. ${ }^{9}$ Similar to MSCs, fibroblasts seem capable of differentiation into adipogenic, osteoblastogenic and chondrogenic lineages. ${ }^{9}$ These insights might suggest that adventitial MSCs and Sca-1+ progenitor cells, previously identified and studied by many groups, are in fact fibroblasts. Indeed, a recent paper by $\mathrm{Ni}$ et al. shows that $10 \%$ of c-Kit+ cells was positive for the fibroblast marker Pdgfro in healthy C57BI6 aorta. ${ }^{29}$ Their findings were confirmed using an inducible Cre model, labeling c-Kit+ cells with TdTomato, showing $20 \%$ overlap between Pdgfra and c-Kit. ${ }^{29}$ Moreover, Tang et al. also reported that $40 \%$ of adventitial Sca-1+ cells with progenitor properties coexpressed Pdgfra. ${ }^{25,} 30$ These Sca-1+/Pdgfr $\alpha+$, progenitor-like cells generated new medial SMCs after severe artery injury. ${ }^{30}$ Similar to other recent studies that assessed vasculature cell populations by SCS, Gu et al. did not annotate mesenchymal clusters in adventitia of $\mathrm{ApoE}^{-/-}$and WT aortas as stem or progenitor cells. ${ }^{18}$ Yet, one of the four identified mesenchyme clusters showed high Sca-1+ expression, indicating stem cell properties of this cluster. ${ }^{18}$ The distinction between true adventitial stem and/or progenitor cells and fibroblasts may thus be smaller than previously assumed, and expression of Sca1+ indicative of fibroblast plasticity.

Together, these data suggest that fibroblasts show an even greater plasticity than previously thought. Cell transitioning of fibroblasts into other cell types and vice versa seems common and extensive in atherosclerosis. Whether all currently identified adventitial stem and/or progenitor cells are really adventitial fibroblasts and vice versa is an important remaining question to be resolved using reliable fibroblast reporter models. Based on this concept, another question is whether the fibroblast is an end stage cell or merely a collection of heterogeneous "in between" cells, actively transitioning between different cell types, or a combination of the two. It would be interesting to study if the acquisition of stem cell-like properties by fibroblasts occurs through dedifferentiation. Assessing the differentiation capacity of the distinct fibroblast clusters into other cell types could also shine some light on this discussion. 


\section{Fibroblast cell-cell communication and its therapeutic potential}

In addition to heterogeneity and function of fibroblasts in the natural development of atherosclerosis, these cells could possibly be used as a new therapeutic approach based on their effect on surrounding inflammatory cells. A pro-inflammatory role of mesenchyme clusters through increased intercellular communication with inflammatory macrophages has been computationally predicted in $\mathrm{ApoE}^{-/-}$adventitia by Gu et al. ${ }^{18} \mathrm{~A}$ recent paper by Mahdavi Gorabi et al. also reviewed the possibility of using MSCs as treatment for atherosclerosis by modulating inflammation. ${ }^{31}$ Multiple studies discussed in this review showed a marked anti-inflammatory effect in murine atherosclerosis by decreased proinflammatory cytokines and NFKB signaling after bone marrow MSC administration. MSC therapy has been studied in clinical trials for diseases such as heart disease, cancer and peripheral artery disease, but not atherosclerosis. It is considered a promising future treatment option, but at the same time its safety and efficacy are questioned. Knowledge regarding precise in vivo mechanisms of action is still lacking and inconsistent results are observed due to cellular heterogeneity of MSCs and a lack of specific markers. ${ }^{32}$ Donor characteristics, culture conditions, method and location of delivery and host receptibility are all factors that can influence MSC therapy efficacy and efficiency. ${ }^{31-33}$ Moreover, risks of malignant transformation and pro-tumorigenic effects of MSCs have been reported. Thus, extensive additional research into improving efficiency and efficacy of MSC therapy is required before considering this a new therapy option.

\section{Conclusions and future research}

This review shows that in contrast to the assumptions the classical dogmas contain, next to SMCs, fibroblasts are ECM-producing cells abundant in the vasculature and involved in atherosclerosis. Fibroblasts comprise a very heterogeneous population due to different cellular origins and an extensive repertoire of possible cell transitions. The origin and fate of fibroblasts in atherosclerotic plaques remains to be elucidated. Due to their heterogeneity, there is a lack of specific markers that encompass the entire population making it difficult to study fibroblast (sub)populations in atherosclerosis. Recent comparisons between fibroblasts and adventitial stem and/or progenitor cells indicate similarities between these cells. Moreover, recent SCS data did not identify any adventitial stem and/or progenitor cell clusters, supporting fibroblast identity of these cells. SCS data did identify multiple fibroblast clusters with differential gene expression and functionality per cluster in healthy and atherosclerotic tissue. Further research into subpopulations of fibroblasts and their different functions is needed to identify specific markers per subpopulation and to determine the contribution of each subpopulation to atherosclerosis. The emergence of SCS provides opportunities to find answers to the remaining questions in an unbiased way. In the future, modulating fibroblast cell communication in atherosclerotic vessels could be useful in battling atherosclerosis from within the plaque. 


\section{6 | Fibroblasts in atherosclerosis}

\section{Key points}

- Fibroblasts are ECM-producing cells abundant in the vasculature and involved in atherogenesis

- Fibroblasts encompass a very heterogeneous population as indicated by SCS data revealing several fibroblast clusters in healthy and atherosclerotic tissue with varying gene expression and function

- Fibroblast identity has been proposed for adventitial progenitor and/or stem cells and should be further investigated.

\section{Acknowledgements}

None

\section{Financial support and sponsorship}

Financial support is received from the Netherlands Scientific Organization (NWO VIDI 91718364 to J.C. Sluimer), and the Dutch Heart Foundation (Dr. Dekker fellowship 2016T060 to JC Sluimer).

\section{Conflicts of interest}

None 


\section{References}

1. Herrington W, Lacey B, Sherliker P, et al. Epidemiology of Atherosclerosis and the Potential to Reduce the Global Burden of Atherothrombotic Disease. Circ Res. 2016;118:535-46.

2. Libby P, Buring JE, Badimon L, et al. Atherosclerosis. Nat Rev Dis Primers. 2019;5:56.

3. Basatemur $\mathrm{GL}$, Jorgensen $\mathrm{HF}$, Clarke $\mathrm{MCH}$, et al. Vascular smooth muscle cells in atherosclerosis. Nat Rev Cardiol. 2019;16:727-744.

4. Li M, Qian M, Kyler K, et al. Endothelial-Vascular Smooth Muscle Cells Interactions in Atherosclerosis. Front Cardiovasc Med. 2018;5:151.

5. Moore KJ, Koplev S, Fisher EA, et al. Macrophage Trafficking, Inflammatory Resolution, and Genomics in Atherosclerosis: JACC Macrophage in CVD Series (Part 2). J Am Coll Cardiol. 2018;72:2181-2197.

6. Shi Y, O'Brien JE, Jr., Fard A, et al. Transforming growth factor-beta 1 expression and myofibroblast formation during arterial repair. Arterioscler Thromb Vasc Biol. 1996;16:1298-305.

7. Sartore S, Chiavegato A, Faggin E, et al. Contribution of adventitial fibroblasts to neointima formation and vascular remodeling: from innocent bystander to active participant. Circ Res. 2001;89:1111-21.

8. Singh $\mathrm{S}$ and Torzewski M. Fibroblasts and Their Pathological Functions in the Fibrosis of Aortic Valve Sclerosis and Atherosclerosis. Biomolecules. 2019;9.

9. Soundararajan $\mathrm{M}$ and Kannan S. Fibroblasts and mesenchymal stem cells: Two sides of the same coin? J Cell Physiol. 2018;233:9099-9109.

10. Ichim TE, O'Heeron $P$ and Kesari S. Fibroblasts as a practical alternative to mesenchymal stem cells. J Transl Med. 2018;16:212.

11. Vanlandewijck M, He L, Mae MA, et al. A molecular atlas of cell types and zonation in the brain vasculature. Nature. 2018;554:475-480.

12. Wirka RC, Wagh D, Paik DT, et al. Atheroprotective roles of smooth muscle cell phenotypic modulation and the TCF21 disease gene as revealed by single-cell analysis. Nat Med. 2019;25:1280-1289.

13. Do NN, Willenborg S, Eckes B, et al. Myeloid Cell-Restricted STAT3 Signaling Controls a Cell-Autonomous Antifibrotic Repair Program. J Immunol. 2018;201:663-674.

14. Guerrero-Juarez CF, Dedhia PH, Jin S, et al. Single-cell analysis reveals fibroblast heterogeneity and myeloid-derived adipocyte progenitors in murine skin wounds. Nat Commun. 2019;10:650.

15. Xie T, Wang Y, Deng N, et al. Single-Cell Deconvolution of Fibroblast Heterogeneity in Mouse Pulmonary Fibrosis. Cell Rep. 2018;22:3625-3640.

16. Gladka MM, Molenaar B, de Ruiter H, et al. Single-Cell Sequencing of the Healthy and Diseased Heart Reveals Cytoskeleton-Associated Protein 4 as a New Modulator of Fibroblasts Activation. Circulation. 2018;138:166-180. 


\section{6 | Fibroblasts in atherosclerosis}

17. Kalluri AS, Vellarikkal SK, Edelman ER, et al. Single-Cell Analysis of the Normal Mouse Aorta Reveals Functionally Distinct Endothelial Cell Populations. Circulation. 2019;140:147-163.

18. Gu W, Ni Z, Tan YQ, et al. Adventitial Cell Atlas of wt (Wild Type) and ApoE (Apolipoprotein E)-Deficient Mice Defined by Single-Cell RNA Sequencing. Arterioscler Thromb Vasc Biol. 2019;39:1055-1071.

19. Evrard SM, Lecce L, Michelis KC, et al. Endothelial to mesenchymal transition is common in atherosclerotic lesions and is associated with plaque instability. Nat Commun. 2016;7:11853.

20. Kramann R, Goettsch C, Wongboonsin J, et al. Adventitial MSC-like Cells Are Progenitors of Vascular Smooth Muscle Cells and Drive Vascular Calcification in Chronic Kidney Disease. Cell Stem Cell. 2016;19:628-642.

21. Dobnikar L, Taylor AL, Chappell J, et al. Disease-relevant transcriptional signatures identified in individual smooth muscle cells from healthy mouse vessels. Nat Commun. 2018;9:4567.

22. Osterreicher $\mathrm{CH}$, Penz-Osterreicher $\mathrm{M}$, Grivennikov $\mathrm{SI}$, et al. Fibroblast-specific protein 1 identifies an inflammatory subpopulation of macrophages in the liver. Proc Natl Acad Sci U S A. 2011;108:308-13.

23. Kahounova Z, Kurfurstova D, Bouchal J, et al. The fibroblast surface markers FAP, anti-fibroblast, and FSP are expressed by cells of epithelial origin and may be altered during epithelial-to-mesenchymal transition. Cytometry A. 2018;93:941-951.

24. Lu YP, Ishiwata T, Kawahara K, et al. Expression of lumican in human colorectal cancer cells. Pathol Int. 2002;52:519-26.

25. Kuwabara JT and Tallquist MD. Tracking Adventitial Fibroblast Contribution to Disease: A Review of Current Methods to Identify Resident Fibroblasts. Arterioscler Thromb Vasc Biol. 2017;37:1598-1607.

26. Kovacic JC, Dimmeler S, Harvey RP, et al. Endothelial to Mesenchymal Transition in Cardiovascular Disease: JACC State-of-the-Art Review. J Am Coll Cardiol. 2019;73:190-209.

27. Lai B, Li Z, He M, et al. Atheroprone flow enhances the endothelial-to-mesenchymal transition. Am J Physiol Heart Circ Physiol. 2018;315:H1293-H1303.

28. $\mathrm{Yu} B$, Chen $\mathrm{Q}$, Le Bras $\mathrm{A}$, et al. Vascular Stem/Progenitor Cell Migration and Differentiation in Atherosclerosis. Antioxid Redox Signal. 2018;29:219-235.

29. Ni Z, Deng J, Potter CMF, et al. Recipient c-Kit Lineage Cells Repopulate Smooth Muscle Cells of Transplant Arteriosclerosis in Mouse Models. Circ Res. 2019;125:223-241.

30. Tang J, Wang H, Huang X, et al. Arterial Sca1(+) Vascular Stem Cells Generate De Novo Smooth Muscle for Artery Repair and Regeneration. Cell Stem Cell. 2020;26:81-96 e4. 31. Mahdavi Gorabi A, Banach M, Reiner Z, et al. The Role of Mesenchymal Stem Cells in Atherosclerosis: Prospects for Therapy via the Modulation of Inflammatory Milieu. J Clin Med. 2019;8. 
32. Lukomska B, Stanaszek L, Zuba-Surma E, et al. Challenges and Controversies in Human Mesenchymal Stem Cell Therapy. Stem Cells Int. 2019;2019:9628536.

33. Musial-Wysocka A, Kot M and Majka M. The Pros and Cons of Mesenchymal Stem Cell-Based Therapies. Cell Transplant. 2019;28:801-812. 



\section{Chapter 7}

Single cell sequencing of fibroblasts reveals transcriptional heterogeneity in murine

\section{vasculature}

van Kuijk K, McCracken IR, Tillie RJHA, Wichers Schreur R, Taylor RS, Dobie R, Temmerman L, Ramachamdran P, Wijnands E, Noels H, Owens GK, Jin H, WilsonKanamori JR, Mees BME, Biessen EAL, Henderson NC, Baker AH, Sluimer JC

In preparation 


\section{Chapter 8}

\section{General discussion}



Atherosclerotic plaque development is characterized by numerous systemic and microenvironmental changes, which constantly challenge all cell types involved. In this thesis, we aimed to investigate cellular responses to intra- and extracellular challenges, e.g. hypoxia, $\mathrm{pH}$ and lipids, on macrophage and fibroblast function involved in atherosclerosis and how this relates to plaque development. Moreover, we sought to gain more insight in adventitial mesenchymal cell heterogeneity. The main findings of this thesis are the following:

1. Chaperone mediated autophagy (CMA) is atheroprotective (Chapter 2). A murine knock-out (KO) model of lysosomal membrane protein LAMP-2A, with diminished CMA, shows aggravated atherosclerosis upon high cholesterol diet administration, while overexpression of human LAMP-2A in mice demonstrates opposite effects. This can be, among other causes, attributed to increased blood lipid levels, insulin resistance and macrophage skewing to a more pro-inflammatory phenotype in KO mice.

2. Macrophage $\mathrm{pH}$ and spare respiration capacity is altered by carbonic anhydrase IX (CAIX) deficiency, but apoptosis and lipid uptake remain unchanged. However, CAIX and its soluble form sCAIX do not predict cardiovascular clinical outcome (Chapter 3).

3. Myeloid prolyl hydroxylase domain protein (PHD) deficiency exhibits isoformspecific effects on atherosclerosis. Knock-out of PHD2 and 3, but not PHD1, shows an increase in macrophage apoptosis both in vitro and in vivo, which is mediated via Hif1 and BNIP3 (Chapter 5). Only myeloid PHD2 deficiency stimulates profibrotic paracrine signaling between macrophages and fibroblasts, resulting in collagen-rich plaques (Chapter 5).

4. Single cell sequencing analysis of adventitial fibroblasts shows transcriptional heterogeneity, already present in healthy adventitia. A core population of fibroblasts gives rise to three transcriptionally different branches, linked to different functions such as growth factor signaling, vascular development and immune regulation (Chapter 7 ).

In this discussion, we will evaluate cellular responses to intra- and extracellular challenges relevant to atherosclerosis development. Throughout this thesis it has become evident that numerous environmental stimuli, e.g. lipids, oxygen and $\mathrm{pH}$, differently influence cellular responses, such as autophagy, lipid handling or apoptosis (Chapter 2, 3 \& 5). Moreover, these stimuli can influence communication between macrophages, smooth muscle cells and fibroblasts and thus plaque dynamics. First, I will discuss the influence of interventions in activity of CMA on atherosclerosis development (Chapter 2). Thereafter, I will highlight the 


\section{8| General discussion}

role of both hypoxia and $\mathrm{pH}$, via PHDs and CAIX respectively, on atherosclerosis (Chapter 3 and 5). Lastly, I will go into the role of fibroblasts in the vasculature and discuss their unexpected, heterogenous properties in murine healthy vasculature (Chapter 7). As all processes, studied in this dissertation, influence atherosclerosis in a different way, I will also discuss how the individual findings impact treatment options available. 


\section{Autophagy in murine and human atherosclerosis}

Cells in the developing plaque can protect themselves against apoptosis by recycling their intracellular content. This process is known as autophagy and has three forms: macroautophagy, micro-autophagy and chaperone-mediated autophagy (CMA). ${ }^{1}$ The latter is extensively discussed in chapter 2 . CMA makes use of a chaperone in the cytosol, heat shock cognate protein 70 (HSC70), which recognize proteins designated for CMA by their KFERQmotif. HSC70 translocates these proteins to the lysosome, where it binds to lysosomeassociated membrane protein type $2 \mathrm{~A}$ (LAMP-2A) leading to lysosomal degradation. ${ }^{2}$ In the liver, it has already been shown that defective CMA in hepatocytes alters their cellular metabolism and leads to hepatosteatosis. ${ }^{3}$ This effect is caused by accumulation of key enzymes involved in glucose and lipid handling, which are normally degraded via CMA. However, major changes in protein homeostasis were not observed. This was drastically changed upon aging, as aged hepatic LAMP-2A KO mice were not able to compensate for the loss in CMA activity anymore. ${ }^{4}$ This resulted in worsened hepatic function and higher susceptibility to lipid induced stress. As CMA is seemingly very important in cellular homeostasis and can be triggered by processes such as oxidative stress or hypoxia, we investigated the role of CMA in atherosclerosis (chapter 2). Abovementioned stressors are abundantly present in the developing plaque and thus could disrupt normal CMA functioning.

Autophagy can be triggered via numerous stressors, including hypoxia, inflammation and lipids. ${ }^{5}$ In chapter $\mathbf{2}$, we show that LAMP-2A KO led to increased plaque size and dramatically elevated blood lipid levels. As normal lipid metabolism is diminished by impaired CMA in the liver, this could lead to the elevated blood lipid levels. This was shown with Oil Red $O$ staining, pinpointing lipid droplets in the liver. ${ }^{3}$ Moreover, we were able to show that smooth muscle cell (SMC) transdifferentiation upon lipid loading was exaggerated in LAMP2A KO mice. Transdifferentiation of SMCs into a macrophage-like cell was proposed to contribute to plaque foam cell content and is linked to decreased plaque stability. ${ }^{6-8}$ In macrophages, the LAMP-2A KO led to skewing into a more pro-inflammatory phenotype with increased inducible nitric oxide synthase (iNOS) and Cyxlo-oxygenase 2 (COX-2) signaling. The induction of iNOS in macrophages has been linked to impaired migratory capacity and is typical for pro-inflammatory M1 macrophages., 10 Moreover, COX-2 has been linked to oxLDL laden macrophages (Mox) and seems important in the initiation of atherosclerotic plaque formation in low-density lipoprotein receptor (LDLR) KO mice. ${ }^{11,12}$ Thus, overall it seems that CMA is normally involved in athero-protective mechanisms.

When analyzing different plaque stages in murine atherosclerosis for L2A expression, its expression decreased upon disease progression (chapter 2). This might be a start of a marked decrease in $\mathrm{CMA}$, as shown upon aging. ${ }^{13}$ Moreover, high dietary lipid intake also negatively influences CMA activity. ${ }^{14}$ Interestingly, LAMP-2A expression was higher in 


\section{8 | General discussion}

human plaques with more advanced disease in asymptomatic patients (chapter 2). This discrepancy could be due to a protecting mechanism that takes place in atherosclerosis, where CMA is upregulated in attempt to rescue plaque phenotype. However, this was a cross-sectional study, and only a longitudinal study with non-invasive imaging of CMA could provide definite insight how vascular CMA changes over time. In contrast to these data in asymptomatic patients, we were able to show significantly lower plaque LAMP-2A protein expression and CMA activity, via expression of its target cathepsin $D$, in patients who subsequently endured a secondary cardiac event compared to patients, who experienced only one event. This effect was however gender specific, as it was only observed in female patients. A similar CMA gender specific effect was also observed in a model of gastric cancer, where LAMP-2A was used as a prognostic marker. ${ }^{15}$ The authors found that the prognostic value of LAMP-2A was restricted to female patients in two independent cohorts. The exact mechanism of this effect remains to be elucidated, but the researchers hint towards a connection between estrogen and LAMP-2A, in terms of CMA activity moderation. A possible explanation for the decreased CMA activity and LAMP-2A expression in relation to a secondary event could be the influence of a lipid-rich dietary lifestyle. Dietary lipids have been shown to influence CMA activity by initiating degradation of LAMP-2A. ${ }^{14}$ This was attributed to the effect of high dietary lipid intake on lysosomal membrane lipid composition and therefore instability of the receptor in the lysosomal membrane. As LAMP-2A is the rate-limiting factor in CMA, this directly affects its activity.

In chapter 2, to mimic a therapeutic setting, we also made use of a human LAMP-2A knockin model to enhance CMA. This model was able to normalize sequelae of hypercholesterolemia. Mice showed an opposite phenotype compared to the LAMP-2A KO mice, including decreased plaque size, decreased necrotic core and improved metabolic profile. Upregulation of CMA could therefore be an interesting target in putting atherosclerosis progression to a halt. There are already some CMA activators being tested in clinical practice, including retinoid acid receptor (RAR) derivatives. Admission of these derivatives in vitro led to an increase in CMA activity, while macro-autophagy was left unharmed. ${ }^{16}$ Another inducer of CMA is endogenous humanin and can protect cells from oxidative stress or starvation. In vivo administration of a single dose of humanin in rats already doubled CMA activity, as measured by lysosomal target uptake. ${ }^{17}$ However, one must be aware that these results mostly comprise of in vitro and limited in vivo data, and further research of capability of these compounds in the in vivo context of atherosclerosis is needed. As our knowledge about the role of CMA in atherosclerosis is limited, future studies concerning CMA activation could be of great interest. Future studies could include administration of CMA activators in models of murine atherosclerosis. These could be given at the start of the study, to prevent atherosclerosis, but another angle, which is probably more interesting for the translation towards human studies, could include administration when plaque is already present. This could provide information about the ability of CMA 
activators to inhibit plaque progression or if they even could induce plaque regression. However, it is still important to note that administration of CMA activators could influence whole body CMA activity rather than local activity. Overactivity of CMA has been linked to pathologies as cancers. ${ }^{15}$ Local administration is therefore needed to omit pathological abnormalities in other tissues. 


\section{8 | General discussion}

\section{Carbonic anhydrase IX and its soluble form sCAIX as biomarkers in CVD}

In atherosclerosis, acidic environment in the growing intima has been proposed as an important factor in inflammation and lipid handling. ${ }^{18}$ Moreover, low plaque $\mathrm{pH}$ has been linked to plaque vulnerability. ${ }^{19}$ Acidic environment and hypoxia often go hand in hand, as hypoxia leads to cellular switching from oxidative phosphorylation to glycolysis. ${ }^{18}$ This switch is mostly observed in immune cells and to be more precise, mostly in macrophages. However, this switch to glycolysis is also observed in normoxic conditions and is known as the Warburg effect. ${ }^{20}$ Carbonic anhydrases are well known for their function in cellular $\mathrm{pH}$ regulation. They facilitate the conversion from $\mathrm{CO}_{2}$ and $\mathrm{H}_{2} \mathrm{O}$ to $\mathrm{HCO}_{3} .^{-21}$ Carbonic anhydrase IX (CAIX) is a subtype, which is hypoxia-inducible and thus is a bridge between $\mathrm{pH}$ regulation and hypoxia. In the cancer field, CAIX is already widely used as a prognostic marker for disease progression, both as blood marker, and as noninvasive PET imaging probe..$^{22-25}$ Moreover, recent studies have been proposing CAIX targeting as treatment in numerous cancers, as CAIX is seemingly involved in radiation resistance. ${ }^{26,27}$ CAIX inhibitors could therefore increase response to radiotherapy and chemotherapy.

In chapter 3, we evaluated the role of CAIX in CVD. Firstly, we were able to show CAIX protein expression in human plaques and its expression co-localized to CD68+ macrophages. As expected, CAIX positive areas were also co-localizing to hypoxic areas, indicated by pimonidazole. Pimonidazole, which binds to hypoxic, but viable, cells, was injected before carotid endarterectomy. ${ }^{28}$ On mRNA level, we showed significant correlations between CAIX and lipid cores, and pro-inflammatory iNOS+ macrophages. The latter may not be surprising as hypoxia thrives macrophage polarization towards a more pro-inflammatory subset. ${ }^{29,30}$

Switching to a murine KO model of CAIX enabled us to evaluate, in depth, the role of CAIX in macrophages in vitro. CAIX deficiency altered $\mathrm{pH}$ and lactate levels compared to wildtype macrophages, as expected. Moreover, cellular metabolism was altered accordingly with an observed decrease in oxidative phosphorylation capacity. As described above, it is known that this alteration encourages macrophages to polarize towards a pro-inflammatory state, however, we were not able to confirm this M1-skewing (chapter 3). CAIX deficiency alone is therefore probably not sufficient as a pro-inflammatory stimulus, and there might be redundancy via other anhydrase, and/or $\mathrm{pH}$ regulators, like $\mathrm{Na}^{+}-\mathrm{H}^{+}$exchanger $1 .{ }^{31}$ Despite significant correlation between CAIX mRNA and lipid cores in human plaques, we were unable to find an effect on lipid uptake and apoptosis in vitro (chapter 3 ). The latter might seem surprising as the role of CAIX in tumor cell apoptosis is well established. ${ }^{32,} 33$ It is important to know that these studies investigated mainly the effect of CAIX inhibition in relation to radiosensitivity or chemosensitivity, but direct cellular effects remain to be elucidated. 
Nevertheless, our in vitro findings were confirmed in human atherosclerotic plaque mRNA analysis, where CAIX mRNA failed to correlate to genes involved in apoptosis (chapter $\mathbf{3}$ ). This immediately highlights a limitation of our study. As correlations were made in total plaque mRNA or bulk RNA, this involves all cell types present in the plaque. In our in vitro study we only focus on macrophages, whose contribution to CAIX mRNA levels may be blunted by other vascular cells. Indeed, we observed expression of CAIX in smooth muscle cells and fibroblasts. More importantly, CAIX expression in pro-inflammatory macrophages could merely be a consequence rather than a cause of atherosclerosis. This supports the known limitations of association studies, and calls for an experimental cause-effect study.

To draw firm conclusions on the role of CAIX in atherosclerosis, a mouse study should be conducted. As CAIX KO mice are already available, a logical approach would be to inject these mice with the proprotein convertase subtilisin/kexin type 9 (PCKS9) adeno-associated virus to induce hypercholesterolemia when fed a high cholesterol diet and thus atherosclerosis development. ${ }^{34}$ Parameters as plaque size, lipid content and necrotic core could be assessed in $\mathrm{KO}$ animals compared to wildtypes. Another approach could be a bonemarrow transplantation of CAIX KO myeloid cells into athero-prone LDLR KO, as macrophages are most sensitive to hypoxia and $\mathrm{pH}$ change in the atherosclerotic plaque. Via this method, we could investigate the influence of myeloid CAIX KO cells on plaque development, and contrast it to the contribution of all cells in the whole body KO.

As we know that hypoxia is already abundantly present in early stage human and murine atherosclerosis and is linked to detrimental plaque characteristics, such as intra-plaque hemorrhage and disrupted lipid handling, it would be interesting to visualize plaque hypoxia. ${ }^{35-37}$ Visualization could improve earlier treatment options. Moreover, research by our group has shown that plaque hypoxia in murine atherosclerosis could be reversed by using hyperoxic carbogen gas. ${ }^{38}$ This led to diminished detrimental plaque traits, e.g. necrotic areas. Plaque hypoxia is therefore a possible attractive therapeutic target. Visualization of plaque hypoxia can be performed using radioactive tracers, including glucose analog ${ }^{18} \mathrm{~F}$-fluorodeoxyglucose $\left({ }^{18} \mathrm{~F}\right.$-FDG) or imonidazole analogs $\left[{ }^{18} \mathrm{~F}\right]-\mathrm{EF} 5$ and $\left[{ }^{18} \mathrm{~F}\right] \mathrm{HX} 4 .{ }^{36,39-41}{ }^{18} \mathrm{~F}-\mathrm{FDG}$ could identify hypoxic macrophages that switched their metabolism to a more glycolytic form, increasing their need for glucose. $.^{29}, 39$ The imonidazole analogs are more directed towards direct targeting of hypoxia, confirmed by ex vivo tumor immunohistochemical staining of CAIX. ${ }^{42}$ For the visualization of CAIX are also direct tracers available, which are mainly used in cancer research. ${ }^{43}$ Other than these imaging techniques, it is currently not possible to estimate plaque hypoxia in a non-invasive manner. As CAIX is not only membrane-bound, but also exists in a soluble form (SCAIX) after shedding from the membrane, this could be another approach in visualizing plaque hypoxic burden. The CAIX part on the outer side of the membrane is shed into the bloodstream via metalloprotease A Disintegrin And Metalloptroteinase-17 (ADAM17), which seems a constant, hypoxia- 


\section{8 | General discussion}

independent process. ${ }^{44}$ sCAIX can be detected in blood and urine, and is already used in cancer prognostic testing. In bladder cancer, sCAIX levels in the urine were indicative of urinary tract tumors, even if these were not diagnosed yet. ${ }^{45}$ This was also true for patients with non-small cell lung cancer, where plasma SCAIX levels were able to distinguish between healthy and cancer patients, and correlated to tumor size. ${ }^{46}$ However, there are some discrepancies between studies as not all cancer-related CAIX studies point towards a direct link between SCAIX and tumor CAIX. For instance, patients with oral squamous cell carcinomas only showed a possible trend associated with patient prognosis. ${ }^{24}$

Thus, in chapter 3, we investigated the potential role of sCAIX as a biomarker in CVD. For this purpose, we made use of the Cohort on Diabetes and Atherosclerosis Maastricht (CODAM) cohort. The CODAM cohort followed patients over the period of 7 years, primarily looking at cardiovascular outcome. Unfortunately, we were unable to show an association between plasma sCAIX levels at baseline and cardiovascular outcome during follow-up. However, it must be noted that the CODAM cohort did not include any atherosclerosis parameters other than carotid intima-media thickness and ankle-arm index measurement. Possibly, plaque burden was only mild in these asymptomatic patients and hence sCAIX too low to predict future events. On the other hand, murine plaque hypoxia was already demonstrated in fatty streaks and in human plaque segments with early lesions. ${ }^{35,}{ }^{38}$ As the segments were in fact shoulder regions of advanced plaques in symptomatic patients, it is yet unknow if human early lesions are in fact hypoxic. This would then be in line with low sCAIX levels.

To address this issue, we also investigated the biomarker role of plaque bound CAIX to analyze its local role in advanced, hypoxia-positive atherosclerosis. We used the AtheroExpress cohort for this purpose, which is comprised of symptomatic patients that underwent carotid endarterectomy. ${ }^{47}$ Follow up was conducted, with secondary cardiac events as a primary outcome. However, we were unable to show a correlation between CAIX protein expression and secondary events, in these advanced plaques (chapter $\mathbf{3}$ ). The lack of Pimonidazole injection, and thus precise correlation to plaque hypoxia, in this cohort prevented absolute confirmation of plaque hypoxia, although very likely. ${ }^{35}$

One of the explanations for the observed low CAIX and SCAIX levels, could be related to the chronic nature of plaque hypoxia. It is important to note that plaques already have persistent intra-plaque hypoxia for a quite long period of time. Chronic hypoxia has been shown to downregulate HIF1 $\alpha$ expression and only HIF2 $\alpha$ is still stabilized. ${ }^{48}$ Another possibility could be that the amount of viable cells compared to acellular mass is limited and therefore (s)CAIX is not produced in large quantities. This could influence hypoxiaresponsive genes, such as CAIX, as CAIX has been shown to be mainly regulated via HIF1 $\alpha$ rather than HIF2 $\alpha .{ }^{49} \mathrm{~A}$ study that could be interesting to validate our hypothesis is the Plaque at RISK (PARISK) study, which included roughly 300 symptomatic patients for MRI 
imaging of carotid plaques..$^{50}$ From these patients, full plaque burden is known through MRI. Also, blood was drawn at the start of the study making it possible to check biomarker potential and its relationship to cardiac event development, 2 years after the start of the study. It would of course be most interesting to investigate the relationship between plasma sCAIX levels and plaque hypoxia. Beforementioned tracers would be very suitably for this, however, to date there has not been a large-scale study conducted from this perspective in regards to CVD. In the cancer field, there are already comparable studies conducted. ${ }^{51}$ Patients are injected with ${ }^{18} \mathrm{~F}-\mathrm{FDG}$ and blood is drawn for serum analysis. This approach could also be very appealing in CVD research as this tracer does not only provide information about plaque hypoxia, but also inflammation. ${ }^{52}$

Together, these data do suggest a role for CAIX in macrophage metabolism and $\mathrm{pH}$ regulation. However, we were unable to prove a prognostic role for SCAIX in the CODAM cohort, nor a predicting role for plaque bound CAIX in the AtheroExpress study. Moreover, macrophage characteristics such as apoptosis or lipid handling, linked to detrimental plaque characteristics as necrotic and lipid cores, were unchanged in CAIX KO macrophages. Together, these data imply that CAIX is merely a consequence rather than a causal factor in atherosclerosis. Moreover, it seems that the prognostic value of SCAIX in terms of atherosclerotic plaque burden is not sufficient to be used in the clinic, especially since (s)CAIX is undetectable in the majority of the tested samples. Further studies to investigate the role of CAIX and SCAIX in atherosclerosis would therefore not add value to our understanding of plaque development and progression at this point. 


\section{8 | General discussion}

\section{PHDs: beyond the scope of oxygen sensing}

The impact of hypoxia in atherosclerosis has been extensively discussed in this thesis. Next to pathological effects of hypoxia, it is also essential for cell and tissue homeostasis. Hypoxia, and to be more precise hypoxia inducible factor 1 (HIF1), is required for erythropoiesis, angiogenesis and cellular metabolism. ${ }^{53}$ On the other hand, pathological activation of HIF1 can lead to severe inflammation via NFKB activation ${ }^{54}$, organ fibrosis via extensive ECM production ${ }^{55}$, or cell death, eventually leading to necrotic areas ${ }^{56}$.

Apoptosis can be triggered by a wide variety of stimuli in the growing atherosclerotic plaque. Hypoxia is a known inducer of apoptosis, however, it has also been shown to inhibit apoptosis and even promote cell proliferation. ${ }^{57}$ Hypoxia, or to be more precise, HIF $\alpha$ stabilization is regulated by prolyl hydroxylase domain proteins (PHD 1,2 \& 3). ${ }^{53}$ In case of low oxygen levels, these PHD enzymes are not able to exert their function leading to HIF $\alpha$ stabilization and translocation to the nucleus. Here it binds to the HIF $\beta$ subunit and leads to transcription of genes involved in numerous processes, such as apoptosis.

In chapter 5, we made use of three mouse models, each deficient in one of the three PHD enzymes in myeloid cells to assess their effect on atherosclerosis. We found that plaque size of PHD2 and 3, but not PHD1 deficient mice, were increased upon high cholesterol diet. As PHD2 and 3 also exhibited an altered local macrophage phenotype by decreased macrophage density and increased necrotic core, respectively, we investigated the role of PHD2 and 3 in macrophage apoptosis. Apoptosis can be induced by activation of the B-cell lymphoma 2 (Bcl-2) family. ${ }^{58}$ This family of proteins consists of both pro- and anti-apoptotic proteins, of which activation or inhibition is environment-dependent. Normally, $\mathrm{Bcl}-2$ would inhibit pro-apoptotic proteins $\mathrm{Bcl}$-2-associated $\mathrm{X}$ protein (Bax), Bcl-2 antagonist/killer-1 (Bak) and $\mathrm{Bcl}-2$ nineteen kilodalton interacting protein (BNIP3), and thereby omit cytochrome $\mathrm{C}$ release from the mitochondria. ${ }^{59}$ Hypoxia is one of the main drivers of BNIP3 induction, as it has an hypoxia responsive element (HRE) in its promotor. ${ }^{60}$ This would then lead to increased $\mathrm{Bcl}-2$ inhibition and thus indirect stimulation of apoptosis. In chapter 5, we were indeed able to prove that myeloid deficiency of both PHD2 and 3 led to increased sensibility to apoptosis in macrophages in vitro and that this was mediated via HIF1 and BNIP3 (Figure 1). 

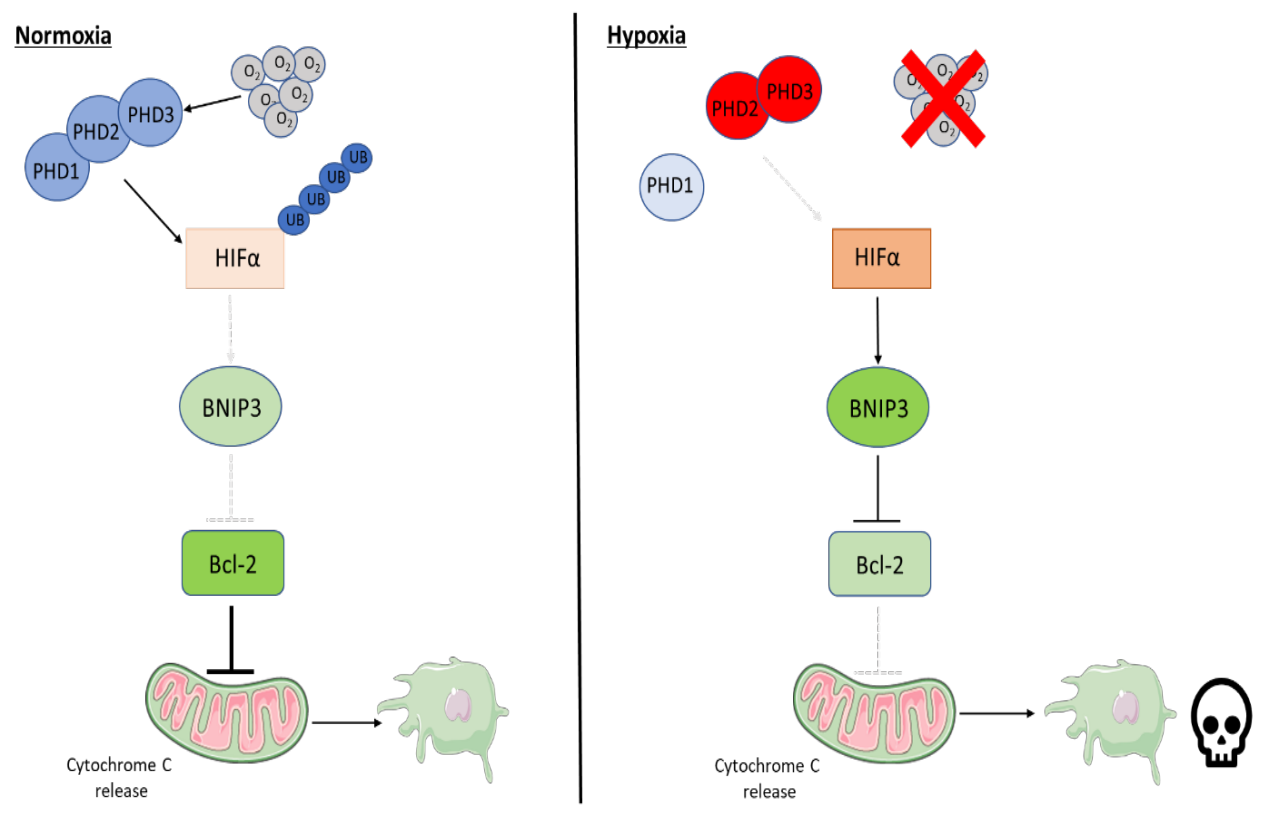

Figure 1. Schematic overview of influence of PHDs on apoptosis.

The link between PHD2 and BNIP3 was also found in another study. ${ }^{61}$ In this study, mice were not crossed to an athero-prone genetic background as was the case for our studies, proving that PHD2 KO alone is already enough to induce BNIP3 expression. However, genetic BNIP3 induction in PHD2 KO alone was not enough to induce macrophage apoptosis in vitro. ${ }^{61}$ In combination with a cholesterol-rich environment, which is evidently the case in atherosclerosis, macrophage apoptosis is stimulated in the KO models. In vitro analysis has shown that stimulation with 7-ketocholesterol increased apoptosis in PHD2 and 3 KO macrophages compared to their wildtype controls (chapter 5 ). These effects were abolished if macrophages of PHD2 and 3 models were cultured under low oxygen conditions, mimicking hypoxia-driven HIF signaling in their respective WT. These findings imply the causative role of hypoxia in apoptosis stimulation. Further confirmation was obtained by using short interference (si)RNAs against both HIF1 $\alpha$ and BNIP3, decreasing cell death in KO macrophages of both PHD2 and 3 (chapter 5). In vivo analysis of intra-plaque apoptosis confirmed increased cell death in PHD2 and 3 KO plaques. However, one must note that these findings include all apoptotic cells and not only macrophages. For this, immunohistochemical analysis should include both a macrophage marker, e.g. CD68, and an apoptosis marker, e.g. TUNEL. Moreover, to support this interaction between BNIP3 and PHD2/3, BNIP3 protein expression could be analyzed in PHD2 and 3 KO plaques. Another validation step for this interaction could be a double myeloid KO mouse model for both PHD2 or PHD3 and BNIP3. An ultimate mouse model could be a triple KO model for PHD2, PHD3 and BNIP3. 


\section{8 | General discussion}

Next to the pro-apoptotic effect of PHD2 and 3 myeloid KO models, we were also able to show a pro-fibrotic effect in PHD2 myeloid KO mice only. In chapter 5, the paracrine communication between macrophages and fibroblasts is highlighted. In vitro stimulation of fibroblasts with conditioned medium from PHD2 KO macrophages stimulated collagen secretion, which was resembled in vivo by fibrotic plaques in PHD2 KO mice. We were able to show paracrine communication between macrophages and fibroblasts, but not SMCs, which was hypoxia-dependent. In silico analysis of both macrophages and fibroblasts highlighted osteopontin (SPP1) as a possible factor, secreted by PHD2 KO macrophages. In the lung, SPP1 has already been linked to fibrosis via an increase of pro-fibrotic genes as collagen type 1 and tissue inhibitor of metalloproteinase 1 (TIMP-1) and decrease of matrix metalloproteinase-1 (MMP-1). ${ }^{62}$ In vivo analysis confirmed an upregulation of SPP1 expression in PHD2 KO plaques, possibly explaining the pro-fibrotic phenotype. In respect to atherosclerosis, SPP1 has been found to aggravate atherosclerosis and KO models had significantly smaller lesions compared to their wildtypes. ${ }^{63}$ To confirm the causal relation between SPP1 and increased plaque size in PHD2 KO plaques, one could study whether PHD2 profibrotic affects are blunted in SPP1 dKO. As SPP1 KO mice already exist, addition of a PHD inhibitor could also elucidate the role of SPP1 in PHD2 KO plaque development. ${ }^{64}$ However, it is important to note that most PHD inhibitors not exclusive, but do have a higher affinity, for PHD2.

Paracrine signaling between immune cells and fibroblasts is new in the field of atherosclerosis, even so is the role of fibroblasts in plaque development. In other processes, e.g. wound healing, communication between fibroblasts and macrophages has already been described. In the dermis, M1 or M2 polarized macrophages differentially affect fibroblasts. M1 macrophages induced a more pro-inflammatory fibroblast with ECM degrading properties, while M2 macrophages showed the induction of an opposite fibroblast phenotype. ${ }^{65}$ Paracrine signaling between immune cells, however, has been described extensively in atherosclerosis, as immune cells are known for secretion of numerous cytokines that affect plaque development and intra-plaque inflammation. ${ }^{66}$ Some of these cytokines, such as transforming growth factor $\beta$ (TGF- $\beta$ ) or platelet derived growth factor B (PDGFB), are known to stimulate mesenchymal cells towards a collagenproducing state. ${ }^{67}$ This was however not the case in our study (Chapter 5). Moreover, in chapter 5 the current dogma of SMCs being the main source of collagen in the plaque ${ }^{68}$ has been challenged, as we provide evidence of fibroblasts being a major contributor here. More attention has been given to fibroblasts, as a recent single cell sequencing study has indicated their presence in plaques. Their transcriptional landscape shares properties with SMCs, and in atherosclerosis it has been shown that SMCs have the ability to become fibroblast-like cells, also known as fibromyocytes. The latter is transcriptionally close to fibroblasts and was found to be modulated via the athero-protective gene TCF21. ${ }^{69}$ Together, these data imply a prominent role for fibroblasts in plaque fibrosis and show that 
myeloid hypoxia, only mediated via PHD2, could influence macrophage-fibroblast communication.

Above stated data on the effects of PHDs in atherogenesis is of great importance, as new PHD inhibitors are developed and tested in clinical trials including chronic kidney disease (CKD) patients. One of the inhibitors, Roxadustat, has already been approved for patient treatment in China. ${ }^{70}$ Most PHD inhibitors show PDH2-selectivity and side effects include pulmonary hypertension and hyperkalemia. ${ }^{71-73}$ Caution should be taken as use of these PHD2 inhibitors could influence vascular fibrosis, as shown in chapter 5. Moreover, endothelial specific KO of PHD2 also led to increased renal fibrosis, accompanied by decreased renal function. ${ }^{74}$ Increased fibrosis is however also linked to a more stable plaque phenotype, as the possibility of plaque rupture is lower. A knock-out model of PHD1 also showed increased hepatic steatosis ${ }^{75}$, however, in our atherosclerosis model we were unable to show a causative role of PHD1 in plaque fibrosis (chapter 5). Our group did show that PHD1 was involved in cholesterol efflux via the intestines and that KO of PHD1 led to decreased blood cholesterol levels. ${ }^{76}$ The variety of effects of PHD inhibition warns researchers about the possible side effects that could occur in CKD patients, which are already at higher risk for atherosclerosis development. ${ }^{77}$ Where PHD1 inhibition seems to be beneficial, PHD2 and 3 inhibition, albeit at immune cell level, seems detrimental for plaque development. Tailored inhibition, in an isotype, disease and cell type specific manner, should be taken into account when designing new PHD inhibitors. 


\section{8 | General discussion}

\section{Fibroblasts: a new key player in the vasculature}

Chapter 5 led us in the direction of fibroblast, an unexpected player in atherosclerosis development. Until recently, fibroblasts were mostly thought to reside in the adventitia. Here, fibroblasts are responsible for extracellular matrix (ECM) production and thus vascular strengthening. ${ }^{78}$ At the time of first observations, no data were available on their presence and function in atherosclerosis. Since then, recent advances using single cell sequencing (SCS) revealed that fibroblasts also inhabit the medial layer, although these findings might be caused by adventitial contamination. ${ }^{79-81}$ Currently used fibroblast markers, such as fibroblast specific protein-1 (FSP-1), collagen type 1 (Col1a1) and fibroblast activation protein (FAP) are not sufficient in distinguishing them from other vascular cells. ${ }^{78}$ Therefore, better and more accurate fibroblast markers are needed to truly investigate their role in the vasculature.

In chapter 7, we investigated the transcriptional landscape of fibroblasts in healthy murine adventitia using SCS. By using the broad mesenchymal marker platelet derived growth factor receptor $\beta$ (Pdgfr $\beta$ ) as a cell selection tool, we were able to isolate both adventitial SMCs and fibroblasts. As good identification markers are scarce, we firstly looked into new markers that could discriminate between SMCs and fibroblasts in healthy vasculature. We were able to find seven markers that were solely expressed in fibroblasts and these markers were validated across three different mouse SCS datasets (chapter 7). We were able to validate six markers on protein level, confirming their adventitial location. One of the markers, Lumican, was also found to be expressed in the media. A recent study by Wirka et al. showed that Lumican is also a marker for modulated SMCs in atherosclerosis, eliminating Lumican as a proper fibroblast marker. ${ }^{69}$ Among our fibroblast markers are some that had already been suggested in literature as fibroblast specific in other organs, e.g. platelet derived growth factor receptor $\alpha$ (Pdgfr $\alpha$ ) in lung. ${ }^{82}$ However, we were able to identify new markers including Di-peptidase 1 (Dpep1) and fibulin-1 (Fbln1). Moreover, we were able to show lack of overlap between SMC marker myosin heavy chain 11 (MYH11) and Pdgfra by using MYH11 reporter mice (chapter 7). These data suggest that Pdgfra is indeed a fibroblast specific marker, both on genetic and protein level, in the healthy adventitia.

However, when analyzing these markers in diseased murine and human vasculature it was shown that none of the markers can still clearly distinguish between fibroblasts and SMCs. Overlap between fibroblast markers Collagen type 14a (COL14A1), Lumican and SPARC Related Modular Calcium Binding 2 (SMOC2) and SMC-marker Transgelin (TgIn) is shown (chapter 7). This overlap illustrates the complexity of using cellular markers in a microenvironment that stimulates cell heterogeneity and plasticity. The cellular phenotype is challenged by continuously changing plaque dynamics. It is also important to note that expression of the various fibroblast markers differs in the atherosclerotic lesion. Markers are not exclusively expressed in the adventitia anymore, but also locate to the intima and 
media. Moreover, not all markers are expressed Where Smoc2 is not expressed in the plaque, Pdgfra, Col14a1 and Lumican are located in the plaque. This stresses that "one marker fits all" is not applicable to fibroblasts. A panel of markers would therefore better cover and identify the fibroblast population. For true confirmation of fibroblast markers, lineage tracing models could be used. By tracing throughout health and disease in the vasculature, the contribution of fibroblasts to atherosclerosis progression, but also their plasticity could be investigated. This would give the opportunity to investigate origin of marker overlap between fibroblasts and SMCs.

Investigating the fibroblast population in our SCS data in more depth, using multiple in silico analysis tools, hinted towards great transcriptional heterogeneity, already in healthy vasculature. These findings might seem surprising in healthy vasculature. However, it could be that this transcriptional diversity or heterogeneity is needed for fibroblast priming for future challenges. In silico analysis showed a core population of fibroblasts and three transcriptionally different trajectories, linked to distinct functions. Functions related to gene expression of the three trajectories included vascular development, immune regulation and growth factor signaling (chapter 7). A concern with SCS is the risk of chance findings in one observation at one timepoint. Therefore, we analyzed another SCS dataset focusing on murine adventitial composition. We could validate these findings, and thus exclude a stochastic effect. ${ }^{83}$ Important to note is the difference in cell numbers, as this study only included about one-third of the amount of cells we were able to include. Increased cell numbers enable the researchers to investigate small subpopulations or, as in our data, transcriptional trajectories that otherwise would have stayed unnoticed.

To investigate the three transcriptional trajectories, markers for each trajectory are needed. In silico analysis provided trajectory-specific markers and transcription factors, driving the transformation of core-fibroblasts into outer population fibroblasts (chapter 7). CD55 is proposed as marker for clusters involved in vascular development, leukemia inhibitory factor receptor ( $\mathrm{Lifr}$ ) for clusters involved in immune regulation and secreted frizzled related protein 1 (sFRP1) for clusters involved in growth factor signaling. CD55 is mostly known for its role in complement activation and lowered expression on immune cells has been linked to coronary artery disease. ${ }^{84,85} \mathrm{~A}$ murine CD55 knock-out model on atheroprone apolipoprotein E (ApoE-/-) background showed marked protection against plaque development. ${ }^{86}$ This went hand in hand with decreased $\alpha$ smooth muscle actin ( $\alpha$ SMA) presence in the plaque, which is not only a SMC marker but also an activated (myo)fibroblast marker. ${ }^{87}$ This possibly then links back to fibroblast presence and collagen production, one of the main functions of myo-fibroblasts.

Lifr can be stimulated via its ligand leukemia inhibitory factor (Lif) and leads to activation of IL-6 signaling. Lif negatively affects atherosclerosis development, but also existing plaque progression, via upregulation of LDLR in the liver and thus lowering blood cholesterol 


\section{8| General discussion}

levels. ${ }^{88}$ Lifr expression is upregulated during vascular injury, but is not affected by Lif administration. During inflammation, fibroblasts can secrete Lif which can act autocrine and upregulate Lifr and IL-6 signaling, via STAT4. ${ }^{89}$ Hereby, fibroblasts can become a more evident cell type in contribution to inflammatory responses.

SFRP1 is mostly known for its antagonistic role in Wnt signaling. ${ }^{90,91}$ In patients experiencing cardiovascular events, systemic SFRP1 levels were markedly higher compared to healthy individuals. ${ }^{92}$ Moreover, decreased Wnt signaling has been linked to increased risk in developing atherosclerosis as it leads to lipid accumulation and increased cholesterol biosynthesis. ${ }^{93}$ In cancer, increased SFRP1 has been shown to correlate to worse patient outcome by inducing TGF- $\beta$ activation, tumor cell proliferation and tumor cell metastasis. ${ }^{94}$, 95 sFRP1 in fibroblasts could be linked to growth factor signaling and thus cellular proliferation, migration and transformation via molecules, such as TGF- $\beta$.

All three markers are known to be expressed by fibroblasts, but are unfortunately not fibroblast specific. This led to the visualization of cells positive for both Pdgfra and trajectory 1 marker, CD55 (chapter 7). Co-localization of Pdgfra and CD55 was shown in the adventitia of both healthy and atherosclerotic murine vasculature. Moreover, double-positivity was also visualized within the plaque implying a role for this trajectory in disease progression. To further analyze the effects of these different fibroblast trajectories on atherosclerosis development, conditional KO mouse models would be required. Pdgfra could be used as a marker to specifically delete one of three trajectory markers in fibroblasts only. This, again, could lead to tailored treatment options where only specific cells are targeted. As mentioned above, ApoE-/- CD55 deficient mice were protected against atherosclerosis. As this model is whole body KO, a fibroblast specific KO could influence plaque development differently. In rheumatoid arthritis, CD55 was also found to be secreted on collagen fibers and prevented inflammatory response. This could led to think that in atherosclerosis, fibroblasts can secrete or enhance their CD55 expression in order to dampen the inflammatory plaque environment. KO models for Lifr and SFRP1 in the context of atherosclerosis are still absent, however, whole body KO for SFRP1 has been shown to induce mesenchymal proliferation in the lung and lead to renal damage through enhanced epithelial-to-mesenchymal transitioning. ${ }^{96,97}$ For Lifr, not much is known in the field of atherosclerosis, but administration of its ligand Lif in a rabbit model for atherosclerosis led to delayed plaque formation. ${ }^{88}$ In an injured artery model in rats, Lifr expression was inversely correlated with Lif infusion. ${ }^{98}$ As Lifr was positively correlated to detrimental plaque traits, such as necrotic core, this could confirm possible negative effects of Lifr on plaques.

Fibroblasts have already been indicated in numerous other organs as plastic cells, being able to adjust their cellular presentation to their microenvironment. Lipid laden lipofibroblasts have already described for years in the lung, and their phenotype can be contributed to 
embryonic expression of transcription factor 21 (Tcf21). ${ }^{99}$ This transcription factor is also involved in the transition of SMCs towards fibroblast-like cells in atherosclerosis. ${ }^{69}$ Other alveolar fibroblast subsets include collagen 13 and 14 (Col13a1 and Col14a1, respectively), involved in ECM production. These subsets increase upon pulmonary fibrosis. ${ }^{82}$ Moreover, fibroblast subsets in arthritis have been shown to induce inflammation and worsen disease outcome. In the context of atherosclerosis, it has been shown that adventitial fibroblasts or mesenchymal progenitor cells can migrate towards the growing plaque and contribute to progression. ${ }^{81,100,101}$ As good distinctive fibroblast markers were lacking, overlap between fibroblasts and mesenchymal stem cells is often the case in previous literature. Our SCS data also shows that most of the adventitial fibroblasts are positive for stem cell markers CD34 and stem cell antigen-1 (Sca-1), confirming their plastic nature (chapter 7). In fact, others have also found that roughly $20 \%$ of stem cell marker c-Kit+ cells are also positive for Pdgfra. ${ }^{102}$ Moreover, around $40 \%$ of adventitial Sca-1+ cells co-express Pdgfra and are capable to produce de-novo SMCs upon vascular injury. ${ }^{103}$ Along that line, Sca-1 has been implied as key factor in thriving neo-intima formation from the adventitial side. ${ }^{101,} 104$ Together these data support fibroblast plasticity, however, to truly investigate their role and contribution to atherosclerosis development a conditional murine knock-out model will be needed, targeting only Pdgfra positive cells. Hereby, their function and role in plaque development could be further elucidated. An ultimate model could include deletion of Pdgfra ${ }^{+} / \mathrm{Sca}-1^{+}$cells to investigate the role of mesenchymal stem cells in atherosclerosis. Moreover, fibroblast-specific KO of one of the three trajectories would be interesting to investigate the role of specific subsets in disease progression. These data could indicate whether there are pro- and anti-angiogenic fibroblasts, differentially affecting atherosclerosis. Analysis of the presence of the three fibroblast subsets in human plaque sections would be needed to translate our findings to humans. Tailored treatment options could include cell specific targeting as future research would point out their pro- or antiangiogenic properties. There is already research conducted using mesenchymal stem cells as treatment in murine atherosclerosis. ${ }^{105,}{ }^{106} \mathrm{~A}$ marked decrease in inflammatory response could be observed. A cautionary note should be taken, as this treatment still needs a lot of improvement before it can be implemented in CVD patients. In murine models, a possible pitfall of treatment with mesenchymal stem cells is tumorgenicity, although this has not been found yet in human studies. ${ }^{105}$ More research is needed to selectively engineer mesenchymal stem cells to be anti-angiogenic and stay at their needed location, the atherosclerotic plaque.

Together, these data show the great transcriptional heterogeneity of fibroblasts. By using SCS, better annotation of fibroblasts has been made possible. Identification of fibroblasts, both in healthy and diseased vasculature, is needed to better understand their contribution to the disease progression. 


\section{8 | General discussion}

\section{Concluding remarks}

In this dissertation, we investigated the role of intra- and extracellular challenges in cellular responses in the context of atherosclerosis. We were able to show diverse effects on macrophage metabolism, inflammatory status and cell death. Moreover, we shed light on a new player in atherosclerosis: the fibroblasts. We extensively discussed the role of fibroblasts in healthy and diseased vasculature. Communication between the two main cell types discussed in this thesis, macrophages and fibroblasts, is also consequently affected by cellular challenges, changing the microenvironment and ultimately plaque phenotype.

This thesis has shed a light on numerous cellular responses to a plethora of intra- and extracellular challenges. In respect to novel treatment options, one could assume that a "one fits all" treatment option is virtually impossible. Nowadays, CKD patients can already receive pan-PHD inhibitors as treatment option. As results from this thesis show that isoform specific PHD inhibition in myeloid cells leads to different systemic and local effects, this should be taken into account when treating CKD patients. These patients are already at increased risk of developing CKD and pan inhibition of all three PHD isoforms could eventually lead to detrimental effects. Current treatment options mostly rely on combatting the symptoms of CVD, however, it would be of great interest to find earlier markers of cardiovascular outcome or to stratify patients with high and low risk of CVE. Unfortunately, we were unable to show predictive value for CVD or CVE of either CAIX or soluble CAIX. Hence CAIX is likely not suited to stratify CKD patients with potentially higher hypoxic burden and susceptibility to disadvantageous effects of PHD inhibitors.

Overall, this thesis shows that numerous intra- and extracellular challenges, such as oxidative stress and excessive lipids, affect cellular responses in a cell-specific manner. Cellcell dynamics are changed accordingly, directly affecting plaque environment and thus atherosclerosis progression. 


\section{References}

1. Hassanpour M, Rahbarghazi R, Nouri M, et al. Role of autophagy in atherosclerosis: foe or friend? J Inflamm (Lond). 2019;16:8.

2. Kaushik $S$ and Cuervo AM. The coming of age of chaperone-mediated autophagy. Nat Rev Mol Cell Biol. 2018;19:365-381.

3. Schneider JL, Suh Y and Cuervo AM. Deficient chaperone-mediated autophagy in liver leads to metabolic dysregulation. Cell Metab. 2014;20:417-32.

4. Schneider JL, Villarroya J, Diaz-Carretero A, et al. Loss of hepatic chaperonemediated autophagy accelerates proteostasis failure in aging. Aging Cell. 2015;14:249-64.

5. Yang X, Li Y, Li Y, et al. Oxidative Stress-Mediated Atherosclerosis: Mechanisms and Therapies. Front Physiol. 2017;8:600.

6. Rong JX, Shapiro M, Trogan E, et al. Transdifferentiation of mouse aortic smooth muscle cells to a macrophage-like state after cholesterol loading. Proc Natl Acad Sci U S A. 2003;100:13531-6.

7. Shankman LS, Gomez D, Cherepanova OA, et al. KLF4-dependent phenotypic modulation of smooth muscle cells has a key role in atherosclerotic plaque pathogenesis. Nat Med. 2015;21:628-37.

8. Wang Y, Dubland JA, Allahverdian S, et al. Smooth Muscle Cells Contribute the Majority of Foam Cells in ApoE (Apolipoprotein E)-Deficient Mouse Atherosclerosis. Arterioscler Thromb Vasc Biol. 2019;39:876-887.

9. Huang $\mathrm{H}$, Koelle $\mathrm{P}$, Fendler $\mathrm{M}$, et al. Induction of inducible nitric oxide synthase (iNOS) expression by oxLDL inhibits macrophage derived foam cell migration. Atherosclerosis. 2014;235:213-22.

10. Xue $Q$, Yan $Y$, Zhang $R$, et al. Regulation of iNOS on Immune Cells and Its Role in Diseases. Int J Mol Sci. 2018;19.

11. Burleigh ME, Babaev VR, Oates JA, et al. Cyclooxygenase-2 promotes early atherosclerotic lesion formation in LDL receptor-deficient mice. Circulation. 2002;105:181623.

12. Parisi L, Gini E, Baci D, et al. Macrophage Polarization in Chronic Inflammatory Diseases: Killers or Builders? J Immunol Res. 2018;2018:8917804.

13. Kiffin $\mathrm{R}$, Kaushik $\mathrm{S}$, Zeng $\mathrm{M}$, et al. Altered dynamics of the lysosomal receptor for chaperone-mediated autophagy with age. J Cell Sci. 2007;120:782-91.

14. Rodriguez-Navarro JA, Kaushik S, Koga $\mathrm{H}$, et al. Inhibitory effect of dietary lipids on chaperone-mediated autophagy. Proc Natl Acad Sci U S A. 2012;109:E705-14.

15. Zhou J, Yang J, Fan X, et al. Chaperone-mediated autophagy regulates proliferation by targeting RND3 in gastric cancer. Autophagy. 2016;12:515-28.

16. Anguiano J, Garner TP, Mahalingam M, et al. Chemical modulation of chaperonemediated autophagy by retinoic acid derivatives. Nat Chem Biol. 2013;9:374-82. 


\section{8 | General discussion}

17. Gong Z, Tasset I, Diaz A, et al. Humanin is an endogenous activator of chaperonemediated autophagy. J Cell Biol. 2018;217:635-647.

18. Oorni K, Rajamaki K, Nguyen SD, et al. Acidification of the intimal fluid: the perfect storm for atherogenesis. J Lipid Res. 2015;56:203-14.

19. Naghavi M, John R, Naguib S, et al. $\mathrm{pH}$ Heterogeneity of human and rabbit atherosclerotic plaques; a new insight into detection of vulnerable plaque. Atherosclerosis. 2002;164:27-35.

20. Palsson-McDermott EM and O'Neill LA. The Warburg effect then and now: from cancer to inflammatory diseases. Bioessays. 2013;35:965-73.

21. Supuran CT. Structure and function of carbonic anhydrases. Biochem J. 2016;473:2023-32.

22. van Kuijk SJ, Yaromina A, Houben R, et al. Prognostic Significance of Carbonic Anhydrase IX Expression in Cancer Patients: A Meta-Analysis. Front Oncol. 2016;6:69.

23. Alves $\mathrm{W}$, Bonatelli $\mathrm{M}$, Dufloth $\mathrm{R}$, et al. CAIX is a predictor of pathological complete response and is associated with higher survival in locally advanced breast cancer submitted to neoadjuvant chemotherapy. BMC Cancer. 2019;19:1173.

24. Eckert AW, Horter S, Bethmann D, et al. Investigation of the Prognostic Role of Carbonic Anhydrase 9 (CAIX) of the Cellular mRNA/Protein Level or Soluble CAIX Protein in Patients with Oral Squamous Cell Carcinoma. Int J Mol Sci. 2019;20.

25. Huizing FJ, Garousi J, Lok J, et al. CAIX-targeting radiotracers for hypoxia imaging in head and neck cancer models. Sci Rep. 2019;9:18898.

26. McDonald PC and Dedhar S. Carbonic anhydrase IX (CAIX) as a mediator of hypoxiainduced stress response in cancer cells. Subcell Biochem. 2014;75:255-69.

27. Ward C, Meehan J, Gray M, et al. Carbonic Anhydrase IX (CAIX), Cancer, and Radiation Responsiveness. Metabolites. 2018;8.

28. Krohn KA, Link JM and Mason RP. Molecular imaging of hypoxia. J Nucl Med. 2008;49 Suppl 2:129S-48S.

29. Fujisaka S, Usui I, Ikutani M, et al. Adipose tissue hypoxia induces inflammatory M1 polarity of macrophages in an HIF-1alpha-dependent and HIF-1alpha-independent manner in obese mice. Diabetologia. 2013;56:1403-12.

30. Marsch E, Sluimer JC and Daemen MJ. Hypoxia in atherosclerosis and inflammation. Curr Opin Lipidol. 2013;24:393-400.

31. Liu CL, Zhang $\mathrm{X}$, Liu J, et al. $\mathrm{Na}(+)-\mathrm{H}(+)$ exchanger 1 determines atherosclerotic lesion acidification and promotes atherogenesis. Nat Commun. 2019;10:3978.

32. Dubois L, Peeters S, Lieuwes NG, et al. Specific inhibition of carbonic anhydrase IX activity enhances the in vivo therapeutic effect of tumor irradiation. Radiother Oncol. 2011;99:424-31.

33. Guttler A, Theuerkorn K, Riemann A, et al. Cellular and radiobiological effects of carbonic anhydrase IX in human breast cancer cells. Oncol Rep. 2019;41:2585-2594. 
34. Bjorklund MM, Hollensen AK, Hagensen MK, et al. Induction of atherosclerosis in mice and hamsters without germline genetic engineering. Circ Res. 2014;114:1684-9.

35. Sluimer JC, Gasc JM, van Wanroij JL, et al. Hypoxia, hypoxia-inducible transcription factor, and macrophages in human atherosclerotic plaques are correlated with intraplaque angiogenesis. J Am Coll Cardiol. 2008;51:1258-65.

36. Silvola JM, Saraste A, Forsback S, et al. Detection of hypoxia by [18F]EF5 in atherosclerotic plaques in mice. Arterioscler Thromb Vasc Biol. 2011;31:1011-5.

37. Parathath S, Mick SL, Feig JE, et al. Hypoxia is present in murine atherosclerotic plaques and has multiple adverse effects on macrophage lipid metabolism. Circ Res. 2011;109:1141-52.

38. Marsch E, Theelen TL, Demandt JA, et al. Reversal of hypoxia in murine atherosclerosis prevents necrotic core expansion by enhancing efferocytosis. Arterioscler Thromb Vasc Biol. 2014;34:2545-53.

39. Folco EJ, Sheikine Y, Rocha VZ, et al. Hypoxia but not inflammation augments glucose uptake in human macrophages: Implications for imaging atherosclerosis with 18fluorine-labeled 2-deoxy-D-glucose positron emission tomography. J Am Coll Cardiol. 2011;58:603-14.

40. van der Valk FM, Sluimer JC, Voo SA, et al. In Vivo Imaging of Hypoxia in Atherosclerotic Plaques in Humans. JACC Cardiovasc Imaging. 2015;8:1340-1.

41. Dubois $L$, Lieuwes NG, Janssen $\mathrm{MH}$, et al. Preclinical evaluation and validation of [18F]HX4, a promising hypoxia marker for PET imaging. Proc Natl Acad Sci U S A. 2011;108:14620-5.

42. Chen L, Zhang Z, Kolb HC, et al. (1)(8)F-HX4 hypoxia imaging with PET/CT in head and neck cancer: a comparison with (1)(8)F-FMISO. Nucl Med Commun. 2012;33:1096-102. 43. Huizing FJ, Hoeben BAW, Franssen GM, et al. Quantitative Imaging of the HypoxiaRelated Marker CAIX in Head and Neck Squamous Cell Carcinoma Xenograft Models. Mol Pharm. 2019;16:701-708.

44. Zatovicova M, Sedlakova O, Svastova E, et al. Ectodomain shedding of the hypoxiainduced carbonic anhydrase IX is a metalloprotease-dependent process regulated by TACE/ADAM17. Br J Cancer. 2005;93:1267-76.

45. HyrsI L, Zavada J, Zavadova Z, et al. Soluble form of carbonic anhydrase IX (CAIX) in transitional cell carcinoma of urinary tract. Neoplasma. 2009;56:298-302.

46. Ilie M, Mazure NM, Hofman V, et al. High levels of carbonic anhydrase IX in tumour tissue and plasma are biomarkers of poor prognostic in patients with non-small cell lung cancer. Br J Cancer. 2010;102:1627-35.

47. Hellings WE, Moll FL, de Kleijn DP, et al. 10-years experience with the AtheroExpress study. Cardiovasc Diagn Ther. 2012;2:63-73.

48. Saxena K and Jolly MK. Acute vs. Chronic vs. Cyclic Hypoxia: Their Differential Dynamics, Molecular Mechanisms, and Effects on Tumor Progression. Biomolecules. 2019;9. 


\section{8 | General discussion}

49. Grabmaier K, MC AdW, Verhaegh GW, et al. Strict regulation of CAIX(G250/MN) by HIF-1alpha in clear cell renal cell carcinoma. Oncogene. 2004;23:5624-31.

50. Truijman MT, Kooi ME, van Dijk AC, et al. Plaque At RISK (PARISK): prospective multicenter study to improve diagnosis of high-risk carotid plaques. Int J Stroke. 2014;9:74754.

51. Yang JH, Maciel RMB, Nakabashi CCD, et al. Clinical utility of 18F-FDG PET/CT in the follow-up of a large cohort of patients with high-risk differentiated thyroid carcinoma. Arch Endocrinol Metab. 2017;61:416-425.

52. Rudd JH, Myers KS, Bansilal S, et al. (18)Fluorodeoxyglucose positron emission tomography imaging of atherosclerotic plaque inflammation is highly reproducible: implications for atherosclerosis therapy trials. J Am Coll Cardiol. 2007;50:892-6.

53. Semenza GL. Regulation of oxygen homeostasis by hypoxia-inducible factor 1 . Physiology (Bethesda). 2009;24:97-106.

54. D'Ignazio L and Rocha S. Hypoxia Induced NF-kappaB. Cells. 2016;5.

55. Darby IA and Hewitson TD. Hypoxia in tissue repair and fibrosis. Cell Tissue Res. 2016;365:553-62.

56. Greijer AE and van der Wall E. The role of hypoxia inducible factor 1 (HIF-1) in hypoxia induced apoptosis. J Clin Pathol. 2004;57:1009-14.

57. Piret JP, Mottet D, Raes M, et al. Is HIF-1alpha a pro- or an anti-apoptotic protein? Biochem Pharmacol. 2002;64:889-92.

58. Youle RJ and Strasser A. The BCL-2 protein family: opposing activities that mediate cell death. Nat Rev Mol Cell Biol. 2008;9:47-59.

59. Brunelle JK and Letai A. Control of mitochondrial apoptosis by the Bcl-2 family. J Cell Sci. 2009;122:437-41.

60. Burton TR and Gibson SB. The role of Bcl-2 family member BNIP3 in cell death and disease: NIPping at the heels of cell death. Cell Death Differ. 2009;16:515-23.

61. Guentsch A, Beneke A, Swain L, et al. PHD2 Is a Regulator for Glycolytic Reprogramming in Macrophages. Mol Cell Biol. 2017;37.

62. Pardo A, Gibson K, Cisneros J, et al. Up-regulation and profibrotic role of osteopontin in human idiopathic pulmonary fibrosis. PLoS Med. 2005;2:e251.

63. Lok ZSY and Lyle AN. Osteopontin in Vascular Disease. Arterioscler Thromb Vasc Biol. 2019;39:613-622.

64. Strom A, Franzen A, Wangnerud C, et al. Altered vascular remodeling in osteopontin-deficient atherosclerotic mice. J Vasc Res. 2004;41:314-22.

65. Ploeger DT, Hosper NA, Schipper M, et al. Cell plasticity in wound healing: paracrine factors of M1/ M2 polarized macrophages influence the phenotypical state of dermal fibroblasts. Cell Commun Signal. 2013;11:29.

66. Tedgui A and Mallat Z. Cytokines in atherosclerosis: pathogenic and regulatory pathways. Physiol Rev. 2006;86:515-81. 
67. Wynn TA and Barron L. Macrophages: master regulators of inflammation and fibrosis. Semin Liver Dis. 2010;30:245-57.

68. Allahverdian S, Chaabane C, Boukais K, et al. Smooth muscle cell fate and plasticity in atherosclerosis. Cardiovasc Res. 2018;114:540-550.

69. Wirka RC, Wagh D, Paik DT, et al. Atheroprotective roles of smooth muscle cell phenotypic modulation and the TCF21 disease gene as revealed by single-cell analysis. Nat Med. 2019;25:1280-1289.

70. Dhillon S. Roxadustat: First Global Approval. Drugs. 2019;79:563-572.

71. Chen N, Hao C, Peng X, et al. Roxadustat for Anemia in Patients with Kidney Disease Not Receiving Dialysis. N Engl J Med. 2019;381:1001-1010.

72. Chen N, Hao C, Liu BC, et al. Roxadustat Treatment for Anemia in Patients Undergoing Long-Term Dialysis. N Engl J Med. 2019;381:1011-1022.

73. Cygulska K, Wejner-Mik P, Plewka M, et al. Roxadustat: another drug that causes pulmonary hypertension? Report of first human case. Pol Arch Intern Med. 2019;129:344345.

74. Wang $\mathrm{S}$, Zeng $\mathrm{H}$, Chen ST, et al. Ablation of endothelial prolyl hydroxylase domain protein-2 promotes renal vascular remodelling and fibrosis in mice. J Cell Mol Med. 2017;21:1967-1978.

75. Thomas A, Belaidi E, Aron-Wisnewsky J, et al. Hypoxia-inducible factor prolyl hydroxylase 1 (PHD1) deficiency promotes hepatic steatosis and liver-specific insulin resistance in mice. Sci Rep. 2016;6:24618.

76. Marsch E, Demandt JA, Theelen TL, et al. Deficiency of the oxygen sensor prolyl hydroxylase 1 attenuates hypercholesterolaemia, atherosclerosis, and hyperglycaemia. Eur Heart J. 2016;37:2993-2997.

77. Valdivielso JM, Rodriguez-Puyol D, Pascual J, et al. Atherosclerosis in Chronic Kidney Disease: More, Less, or Just Different? Arterioscler Thromb Vasc Biol. 2019;39:19381966.

78. Kuwabara JT and Tallquist MD. Tracking Adventitial Fibroblast Contribution to Disease: A Review of Current Methods to Identify Resident Fibroblasts. Arterioscler Thromb Vasc Biol. 2017;37:1598-1607.

79. Kalluri AS, Vellarikkal SK, Edelman ER, et al. Single-Cell Analysis of the Normal Mouse Aorta Reveals Functionally Distinct Endothelial Cell Populations. Circulation. 2019;140:147-163.

80. Evrard SM, Lecce L, Michelis KC, et al. Endothelial to mesenchymal transition is common in atherosclerotic lesions and is associated with plaque instability. Nat Commun. 2016;7:11853.

81. Kramann R, Goettsch C, Wongboonsin J, et al. Adventitial MSC-like Cells Are Progenitors of Vascular Smooth Muscle Cells and Drive Vascular Calcification in Chronic Kidney Disease. Cell Stem Cell. 2016;19:628-642. 


\section{8 | General discussion}

82. Xie T, Wang Y, Deng N, et al. Single-Cell Deconvolution of Fibroblast Heterogeneity in Mouse Pulmonary Fibrosis. Cell Rep. 2018;22:3625-3640.

83. Gu W, Ni Z, Tan YQ, et al. Adventitial Cell Atlas of wt (Wild Type) and ApoE (Apolipoprotein E)-Deficient Mice Defined by Single-Cell RNA Sequencing. Arterioscler Thromb Vasc Biol. 2019;39:1055-1071.

84. Dho SH, Lim JC and Kim LK. Beyond the Role of CD55 as a Complement Component. Immune Netw. 2018;18:e11.

85. Mishra N, Mohata M, Narang R, et al. Altered Expression of Complement Regulatory Proteins CD35, CD46, CD55, and CD59 on Leukocyte Subsets in Individuals Suffering From Coronary Artery Disease. Front Immunol. 2019;10:2072.

86. Lewis RD, Perry MJ, Guschina IA, et al. CD55 deficiency protects against atherosclerosis in ApoE-deficient mice via C3a modulation of lipid metabolism. Am J Pathol. 2011;179:1601-7.

87. Baum J and Duffy HS. Fibroblasts and myofibroblasts: what are we talking about? J Cardiovasc Pharmacol. 2011;57:376-9.

88. Rolfe BE, Stamatiou S, World CJ, et al. Leukaemia inhibitory factor retards the progression of atherosclerosis. Cardiovasc Res. 2003;58:222-30.

89. Nguyen HN, Noss EH, Mizoguchi F, et al. Autocrine Loop Involving IL-6 Family Member LIF, LIF Receptor, and STAT4 Drives Sustained Fibroblast Production of Inflammatory Mediators. Immunity. 2017;46:220-232.

90. Bovolenta P, Esteve P, Ruiz JM, et al. Beyond Wht inhibition: new functions of secreted Frizzled-related proteins in development and disease. J Cell Sci. 2008;121:737-46.

91. Kawano $Y$ and Kypta R. Secreted antagonists of the Wnt signalling pathway. J Cell Sci. 2003;116:2627-34.

92. Ress C, Paulweber M, Goebel G, et al. Circulating Wnt inhibitory factor 1 levels are associated with development of cardiovascular disease. Atherosclerosis. 2018;273:1-7.

93. Boucher P, Matz RL and Terrand J. atherosclerosis: gone with the Wnt? Atherosclerosis. 2020;301:15-22.

94. Qu Y, Ray PS, Li J, et al. High levels of secreted frizzled-related protein 1 correlate with poor prognosis and promote tumourigenesis in gastric cancer. Eur $J$ Cancer. 2013;49:3718-28.

95. Fukuhara K, Kariya $M$, Kita $M$, et al. Secreted frizzled related protein 1 is overexpressed in uterine leiomyomas, associated with a high estrogenic environment and unrelated to proliferative activity. J Clin Endocrinol Metab. 2002;87:1729-36.

96. Foronjy R, Imai K, Shiomi T, et al. The divergent roles of secreted frizzled related protein-1 (SFRP1) in lung morphogenesis and emphysema. Am J Pathol. 2010;177:598-607. 97. Matsuyama M, Nomori A, Nakakuni K, et al. Secreted Frizzled-related protein 1 (Sfrp1) regulates the progression of renal fibrosis in a mouse model of obstructive nephropathy. J Biol Chem. 2014;289:31526-33. 
98. World CJ, Rolfe BE and Campbell JH. Regulation of LIF receptor expression in vascular smooth muscle. Ann N Y Acad Sci. 2001;947:323-8.

99. Park J, Ivey MJ, Deana Y, et al. The Tcf21 lineage constitutes the lung lipofibroblast population. Am J Physiol Lung Cell Mol Physiol. 2019;316:L872-L885.

100. Shi Y, O'Brien JE, Fard A, et al. Adventitial myofibroblasts contribute to neointimal formation in injured porcine coronary arteries. Circulation. 1996;94:1655-64.

101. Hu Y, Zhang Z, Torsney E, et al. Abundant progenitor cells in the adventitia contribute to atherosclerosis of vein grafts in ApoE-deficient mice. J Clin Invest. 2004;113:1258-65.

102. Ni Z, Deng J, Potter CMF, et al. Recipient c-Kit Lineage Cells Repopulate Smooth Muscle Cells of Transplant Arteriosclerosis in Mouse Models. Circ Res. 2019;125:223-241.

103. Tang J, Wang H, Huang X, et al. Arterial Sca1(+) Vascular Stem Cells Generate De Novo Smooth Muscle for Artery Repair and Regeneration. Cell Stem Cell. 2020;26:81-96 e4. 104. Kokkinopoulos I, Wong MM, Potter CMF, et al. Adventitial SCA-1(+) Progenitor Cell Gene Sequencing Reveals the Mechanisms of Cell Migration in Response to Hyperlipidemia. Stem Cell Reports. 2017;9:681-696.

105. Mahdavi Gorabi A, Banach M, Reiner Z, et al. The Role of Mesenchymal Stem Cells in Atherosclerosis: Prospects for Therapy via the Modulation of Inflammatory Milieu. J Clin Med. 2019;8.

106. Musial-Wysocka A, Kot M and Majka M. The Pros and Cons of Mesenchymal Stem Cell-Based Therapies. Cell Transplant. 2019;28:801-812. 



\section{Chapter 9}

\section{Summary}

Samenvatting 



\section{Summary}

Cardiovascular diseases (CVD) are still the leading cause of deaths worldwide. Atherosclerosis is the main driving factor behind CVD, characterized by vascular lipid accumulation, inflammation and collagen deposition. These factors lead to the formation of an atherosclerotic plaque, which can eventually rupture and cause a blood clot. This in turn can cause obstruction of blood flow, followed by myocardial infarction or stroke. During plaque development, there is a balance between plaque stabilizing and destabilizing properties. This balance can be challenged by environmental stressors, such as lipids, and/or hypoxia. Macrophages can take up lipids and will become apoptotic, forming the necrotic core, a destabilizing characteristic. On the other hand, mesenchymal cells can produce extracellular matrix, a stabilizing characteristic. An imbalance in these characteristics could directly influence plaque phenotype and thus the risk of plaque rupture. In this dissertation we examined the influence of intra- and extracellular challenges on cellular responses of macrophages and fibroblasts, such as extracellular matrix production or cell death, in atherosclerosis.

In chapter 2, we investigated the role of chaperone mediated autophagy (CMA) in atherosclerosis. Here we made use of mouse models with either an overexpression or deficiency of the rate limiting enzyme for CMA, lysosome-associated membrane protein $2 \mathrm{~A}$ (LAMP2A). Deficiency of LAMP2A led to more pro-inflammatory immune cells and dedifferentiation of smooth muscle cells, while overexpressed LAMP2A showed an opposite, atheroprotective phenotype. Interestingly, we found that low LAMP2A expression human atherosclerotic plaques was associated with the development of a secondary cardiac event. Therefor we believe that CMA could be a novel target in treating cardiovascular disease.

As plaques are known the be hypoxic, we investigated multiple hypoxia signaling pathways in atherosclerosis (Chapter $\mathbf{3}$ and $\mathbf{5}$ ). Firstly, we discuss a potential role for hypoxia-sensor carbonic anhydrase IX (CAIX) as biomarker for cardiovascular disease in chapter 3. CAIX knock-out in macrophages led to an imbalance in $\mathrm{pH}$ and lactate, which is in line with its function as $\mathrm{pH}$ regulator. However, we were unable to show any additional effects of CAIX deficiency on macrophage phenotype. Detection of CAIX and its soluble form SCAIX in plaques and plasma, respectively, was only possible in a limited number of samples, but did not associate with CVD or cardiovascular events. Hence, these data suggest that (s)CAIX is not a suitable biomarker for CVD.

In chapter 4, we discuss a fast emerging technique in cardiovascular research: single cell sequencing (SCS). Among other subjects, we touch upon cell isolation methods, sequencing method selection, capturing cellular heterogeneity and current pitfalls in regards to SCS. 
The link between hypoxia and atherosclerosis is further explored in chapter $\mathbf{5}$. Here we made use of myeloid prolyl hydroxylase domain proteins (PHDs) deficiency models to investigate their role on atherosclerosis development. PHD2 and PHD3, but not PHD1, knock-outs led to increased plaque size and higher susceptibility of macrophages to cell death. Moreover, we observed extensive fibrosis in plaques from PHD2 deficient mice. Cross-talk between macrophages and fibroblasts triggered the latter cell type to produce excessive amounts of collagen. These data show isoform-specific effects on myeloid cells and atherosclerosis. As PHD inhibitors are currently being prescribed to chronic kidney disease patients, more investigation would be needed to explore harmful effects on the cardiovascular system.

We further introduce fibroblasts and their role in atherosclerosis in chapter $\mathbf{6}$. Fibroblast heterogeneity and, as a consequence of that, the lack of specific cellular markers makes identification difficult. In this chapter we discuss their presence, plasticity, origin and cellcell communication in healthy and atherosclerotic vasculature.

In chapter 7, we evaluated fibroblast heterogeneity in depth using SCS. Firstly, we could successfully distinguish fibroblasts from smooth muscle cells. This led to a fibroblast-specific signature of seven markers. Moreover, we were able to identify distinct fibroblast subsets, already present in healthy vasculature. These subsets were linked to different functions, such as vascular development, immune regulation and growth factor signaling. Each subset showed distinct correlation patterns to beneficial and detrimental plaque traits in human plaques. Therefore we think that these subsets could be of interest in the treatment of atherosclerosis.

Finally, we put all our findings in the broader perspective of atherosclerosis and cell specific responses in chapter $\mathbf{8}$. We discuss the numerous intra- and extracellular challenges touched upon in this dissertation and how they influence atherosclerosis development. Firstly, we discussed the potential beneficial role of CMA stimulation in atherosclerosis. Next, we evaluated whether hypoxia-sensor CAIX could serve as a potential biomarker in CVD. Then in the broader perspective of hypoxia, we discuss the differential role of each PHD isoform in atherosclerosis and more specifically, the role of PHD2 in pro-fibrotic paracrine communication. Lastly, we evaluated the role of fibroblasts in healthy and diseased vasculature and their great heterogeneity as explored by single cell sequencing. 


\section{Samenvatting}

Hart- en vaatziekten zijn nog steeds de meest voorkomende doodsoorzaak wereldwijd. Aderverkalking, de drijfveer achter hart- en vaatziekten, wordt gekarakteriseerd door vasculaire vet accumulatie, inflammatie en extracellulaire matrix productie. Deze factoren leiden tot de vorming van een zogenoemde plaque in de vaatwand. De plaque kan uiteindelijk scheuren en leiden tot de formatie van een bloedpropje, wat vervolgens kan leiden tot een hart- of herseninfarct. Tijdens de ontwikkeling van een plaque is er een balans tussen stabiliserende en destabiliserende factoren. Deze balans kan worden verstoord door omgevingsfactoren, zoals lipiden of hypoxie. Macrofagen kunnen lipiden opnemen, wat celdood kan veroorzaken. Dit leidt vervolgens tot de formatie van een necrotische gebied in de plaque, een destabiliserende factor. Echter mesenchymale cellen kunnen extracellulaire matrix produceren, wat de plaque stabiliseert. Een disbalans in deze karakteristieken kan dus direct het plaque fenotype beïnvloeden en daarmee ook het risico op het scheuren van een plaque. In dit proefschrift hebben we gekeken naar de rol van intra- en extracellulaire stressors op cellulaire responses van macrofagen en fibroblasten, zoals extracellulaire matrix productie of celdood, in aderverkalking.

In hoofdstuk 2 hebben we gekeken naar de rol van chaperon-gemedieerde autofagie (CMA) in aderverkalking. We hebben gebruik gemaakt van muis modellen die ofwel een overexpressie ofwel een deficiëntie hadden van het essentiële CMA enzym, LAMP2A. Defect LAMP2A leidde tot meer pro-inflammatoire immuun cellen en de-differentiatie van gladde spiercellen. Over-expressie van LAMP2A leidde daarentegen tot een tegenovergesteld, beschermend fenotype. Daarbij vonden we dat LAMP2A expressie in humane plaques correleerde aan de ontwikkeling van een tweede manifestatie gerelateerd aan hart- en vaatziekten. Deze bevindingen doen ons geloven dat CMA een nieuw target zou kunnen zijn voor de behandeling van hart- en vaatziekten.

Het is algemeen bekend dat plaques zuurstofarm zijn, ook wel hypoxisch genoemd. In dit proefschrift hebben we meerdere aspecten bekeken met betrekking tot hypoxie (hoofdstuk 3 en 5). Eerst bespreken we een mogelijke rol voor zuurstof-sensor carboanhydrase IX (CAIX) als voorspellende bio marker in hart- en vaatziekten in hoofdstuk 3. CAIX deficiëntie in macrofagen leidde tot een disbalans in $\mathrm{pH}$ en lactaat. Dit is in overeenkomst met de literatuur over de rol van CAIX in $\mathrm{pH}$ regulatie. Detectie van CAIX en de circulerende variant sCAIX in plaques en plasma, respectievelijk, was alleen mogelijk in een klein deel van het patiëntcohort en correleerde niet met hart- en vaatziekten of de manifestatie daarvan. Daarom concludeerden we dat (s)CAIX geen goede bio marker is in hart- en vaatziekten. 


\section{9 | Summary - Samenvatting}

In hoofdstuk 4 bespreken we een nieuwe techniek in het veld van hart- en vaatziekten, single cell sequencing (SCS). In dit hoofdstuk bediscussiëren we onder andere, cel isolatie methodes, het kiezen van de juiste sequencing methode, heterogeniteit en mogelijke valkuilen op het gebied van SCS.

De relatie tussen hypoxie en aderverkalking is nog verder onderzocht in hoofdstuk $\mathbf{5}$. Hier hebben we gebruik gemaakt van drie muismodellen die, enkel in myeloïde cellen, deficiënt zijn voor zuurstofsensoren prolyl hydroxylase domein eiwitten (PHD's). Vervolgens hebben we gekeken naar het effect van deze individuele deficiënties op aderverkalking. PHD2 en PHD3, maar niet PHD1, deficiëntie leidde tot grotere plaques en hogere vatbaarheid voor celdood onder macrofagen. Daarnaast zagen we grote hoeveelheden collageen in plaques van PHD2 deficiënte muizen. Communicatie tussen macrofagen en fibroblasten zorgde ervoor dat fibroblasten meer collageen produceerden. Deze data laten zien dat effecten van PHD's op aderverkalking isovorm-specifiek zijn. Inmiddels worden PHD remmers voorgeschreven aan patiënten met chronisch nierfalen en onze studie wijst uit dat er meer onderzoek gedaan moet worden om nadelige effecten op het hart- en vaatstelsel te voorkomen.

We verdiepen ons meer in de rol van fibroblasten in aderverkalking in hoofdstuk 6. Identificatie van fibroblasten is erg gecompliceerd: door hun heterogeniteit ontbreken goede cellulaire markers. In dit hoofdstuk bespreken we fibroblast aanwezigheid, plasticiteit, oorsprong en communicatie in gezonde en zieke vaten.

In hoofdstuk 7, evalueren we fibroblast heterogeniteit met behulp van SCS. Allereerst laten we zien dat het mogelijk is fibroblasten van gladde spiercellen te onderscheiden middels zeven specifieke markers. Daarnaast hebben we fibroblast subgroepen geïdentificeerd welke al aanwezig zijn in gezonde vaten. Diverse functies, zoals vaatontwikkeling, immuun regulatie en signalering van groeifactoren, konden aan deze subgroepen gelinkt worden. leder subgroep had verschillende correlaties met humane plaque karakteristieken, zoals plaque grootte. Dit laat zien dat deze subgroepen wellicht interessant kunnen zijn bij de behandeling van aderverkalking.

Uiteindelijk hebben we al onze bevindingen gebundeld en in een breder perspectief ten opzichte van aderverkalking gezet in hoofdstuk 8 . We bediscussiëren hier verschillende intra- en extracellulaire stressors en hoe deze aderverkalking zouden kunnen beïnvloeden. Eerst bespreken we de mogelijk positieve rol van CMA in aderverkalking. Daarna evalueren we de capaciteit van zuurstofsensor CAIX als bio marker in hart- en vaatziekten. Daaropvolgend bespreken we de rol van hypoxie in aderverkalking in een bredere context en de rol van elke PHD isovorm hierin. De rol van PHD2 in het vooroorzaken van een fibrotisch plaque fenotype wordt hierin extra uitgelicht. Als laatste zullen we de rol van 308 
Summary - Samenvatting | 9

fibroblasten in gezond en ziek vaatweefsel bespreken en hoe fibroblast heterogeniteit hierin een rol speelt. 

Chapter 10

Impact 

Cardiovascular disease (CVD) is globally still the number one cause of death, with a number as high as 17.9 million deaths in 2017. ${ }^{1}$ The global burden of CVD is not limited to health issues, but also affects economic status. Costs within the European Union for CVD and its clinical manifestations were estimated at $€ 210$ billion a year, in 2015 . $^{2}$ One of the main causes of CVD is atherosclerosis, which is affected by numerous risk factors such as diet, sedentary lifestyle and smoking. In this thesis, we investigated numerous cellular stressors and how they affected atherosclerosis development. In this chapter, we will put these findings in scientific and social perspective.

As the numbers above already state, improvement of CVD treatment is highly necessary. Treatment options nowadays include lipid lowering drugs, blood pressure medication or invasive removal of the atherosclerotic plaques by surgery. By investigating different intraand extracellular challenges in the context of atherosclerosis, we were able to draw conclusions from different environmental states on plaque development. Hypoxia is known to be linked to plaque instability and thus the risk of plaque rupture. ${ }^{3}$ Regulation of hypoxia sensors prolyl hydroxylase domain proteins (PHD) 1, 2 and 3 (Chapter 5) in immune cells led to different outcomes on atherosclerosis. Here, we show that PHD2 and 3 inhibition might negatively affect atherosclerosis as plaque size was increased, as well as cell death and fibrosis in case of PHD2. Chronic kidney disease patients are already receiving pan-PHD inhibitors for treatment of anemia. This could potential harm them, as they are already at risk for CVD. Cell and PHD-specific inhibition could however be of interest as PHD1 inhibition led to decreased cholesterol levels in mice. ${ }^{4}$ Next to PHDs, we also looked into carbonic anhydrase IX (CAIX) which is a $\mathrm{pH}$ regulator and hypoxia related enzyme (Chapter 3 ). CAIX could however not be correlated to cardiovascular outcome, nor did it affect immune cell phenotype in relation to atherosclerosis. Hence, we concluded that CAIX itself would not be of interest as a biomarker for CVD. From these findings regarding hypoxia, we can conclude that more research is needed in tailoring hypoxia targeting and lowering plaque vulnerability.

Chaperone mediated autophagy (CMA), a key process in cell homeostasis, was shown to be protective against atherosclerosis (chapter 2). Modulation of CMA could therefore be a potential new target in atherosclerosis treatment. Activation can be triggered via endogenous activators such as humanin or retinoic acid antagonists. ${ }^{5,6}$ By activating CMA in atherosclerosis, cell homeostasis in the plaque could be improved leading to a more beneficial plaque environment. Our studies only take into account the effect of CMA activation or inhibition before onset of atherosclerosis, but it would of course be more valuable for the clinic to investigate this when plaques are already present. This would resemble the situation of patients in the clinic, as they only see a doctor when symptoms occur.

We also discuss a relatively new player in atherosclerosis, the fibroblast (Chapters 6 \& 7). We show the extent heterogeneity of fibroblasts, which could be of interest when targeting them. As of now, little is known about the contribution of fibroblasts to atherosclerosis. 


\section{0 | Impact}

Therefore, further studies would be needed to investigate their exact role. Fluorescent reporter mice could be a good approach for this, as they nicely visualize presence and origin in health and disease. Another option could be to directly target fibroblasts and initiate their depletion in the vascular wall to investigate their function.

A key scientific method that we have used in this dissertation is single cell RNA sequencing (scRNA-seq). scRNA-seq has been emerging in the field of atherosclerosis as it allows researchers to evaluate cells in depth based on gene expression. It has been essential in identifying small subsets present in the vasculature or to map cellular participants in disease..$^{7-9}$ By using scRNA-seq in this thesis, we were able to visualize fibroblast heterogeneity in healthy adventitia and could show distinct subsets. In the future, scRNAseq could potentially be the golden standard when investigating CVD or any other disease. The amount of genetic data and the ability to investigate cell-cell communication could potentially lead to new discoveries in disease mechanisms and hence also possible treatment options.

The broad data presented in this dissertation shows the importance of numerous cellular responses in atherosclerosis. It gives a plethora of possibilities to target CVD and to improve therapeutics in the future 


\section{References}

1. Roth GA, Johnson C, Abajobir A, Abd-Allah F, Abera SF, Abyu G, Ahmed M, Aksut B, Alam T, Alam K, Alla F, Alvis-Guzman N, Amrock S, Ansari H, Arnlov J, Asayesh H, Atey TM, Avila-Burgos L, Awasthi A, Banerjee A, Barac A, Barnighausen T, Barregard L, Bedi N, Belay Ketema E, Bennett D, Berhe G, Bhutta Z, Bitew S, Carapetis J, Carrero JJ, Malta DC, Castaneda-Orjuela CA, Castillo-Rivas J, Catala-Lopez F, Choi JY, Christensen H, Cirillo M, Cooper L, Jr., Criqui M, Cundiff D, Damasceno A, Dandona L, Dandona R, Davletov K, Dharmaratne S, Dorairaj P, Dubey M, Ehrenkranz R, El Sayed Zaki M, Faraon EJA, Esteghamati A, Farid T, Farvid M, Feigin V, Ding EL, Fowkes G, Gebrehiwot T, Gillum R, Gold A, Gona P, Gupta R, Habtewold TD, Hafezi-Nejad N, Hailu T, Hailu GB, Hankey G, Hassen HY, Abate KH, Havmoeller $R$, Hay SI, Horino $M$, Hotez PJ, Jacobsen $K$, James $S$, Javanbakht $M$, Jeemon $P$, John D, Jonas J, Kalkonde $Y$, Karimkhani C, Kasaeian A, Khader Y, Khan A, Khang YH, Khera S, Khoja AT, Khubchandani J, Kim D, Kolte D, Kosen S, Krohn KJ, Kumar GA, Kwan GF, Lal DK, Larsson A, Linn S, Lopez A, Lotufo PA, El Razek HMA, Malekzadeh R, Mazidi M, Meier T, Meles KG, Mensah G, Meretoja A, Mezgebe H, Miller T, Mirrakhimov E, Mohammed S, Moran AE, Musa KI, Narula J, Neal B, Ngalesoni F, Nguyen G, Obermeyer CM, Owolabi M, Patton G, Pedro J, Qato D, Qorbani M, Rahimi K, Rai RK, Rawaf S, Ribeiro A, Safiri S, Salomon JA, Santos I, Santric Milicevic M, Sartorius B, Schutte A, Sepanlou S, Shaikh MA, Shin MJ, Shishehbor M, Shore H, Silva DAS, Sobngwi E, Stranges S, Swaminathan S, Tabares-Seisdedos R, Tadele Atnafu N, Tesfay F, Thakur JS, Thrift A, Topor-Madry R, Truelsen T, Tyrovolas S, Ukwaja KN, Uthman O, Vasankari T, Vlassov V, Vollset SE, Wakayo T, Watkins D, Weintraub R, Werdecker A, Westerman R, Wiysonge CS, Wolfe C, Workicho A, Xu G, Yano Y, Yip P, Yonemoto N, Younis M, Yu C, Vos T, Naghavi M, Murray C. Global, Regional, and National Burden of Cardiovascular Diseases for 10 Causes, 1990 to 2015. J Am Coll Cardiol 2017;70:1-25.

2. Timmis A, Townsend N, Gale CP, Torbica A, Lettino M, Petersen SE, Mossialos EA, Maggioni AP, Kazakiewicz D, May HT, De Smedt D, Flather M, Zuhlke L, Beltrame JF, Huculeci R, Tavazzi L, Hindricks G, Bax J, Casadei B, Achenbach S, Wright L, Vardas P, European Society of C. European Society of Cardiology: Cardiovascular Disease Statistics 2019. Eur Heart J 2020;41:12-85.

3. Marsch E, Sluimer JC, Daemen MJ. Hypoxia in atherosclerosis and inflammation. Curr Opin Lipidol 2013;24:393-400.

4. Marsch E, Demandt JA, Theelen TL, Tullemans BM, Wouters K, Boon MR, van Dijk TH, Gijbels MJ, Dubois LJ, Meex SJ, Mazzone M, Hung G, Fisher EA, Biessen EA, Daemen MJ, Rensen PC, Carmeliet P, Groen AK, Sluimer JC. Deficiency of the oxygen sensor prolyl hydroxylase 1 attenuates hypercholesterolaemia, atherosclerosis, and hyperglycaemia. Eur Heart J 2016;37:2993-2997.

5. Gong Z, Tasset I, Diaz A, Anguiano J, Tas E, Cui L, Kuliawat R, Liu H, Kuhn B, Cuervo AM, Muzumdar R. Humanin is an endogenous activator of chaperone-mediated autophagy. J Cell Biol 2018;217:635-647.

6. Anguiano J, Garner TP, Mahalingam M, Das BC, Gavathiotis E, Cuervo AM. Chemical modulation of chaperone-mediated autophagy by retinoic acid derivatives. Nat Chem Biol 2013;9:374-382. 


\section{0 | Impact}

7. Winkels $H$, Ehinger $E$, Vassallo $M$, Buscher $K$, Dinh $H Q$, Kobiyama K, Hamers $A A J$, Cochain C, Vafadarnejad E, Saliba AE, Zernecke A, Pramod AB, Ghosh AK, Anto Michel N, Hoppe N, Hilgendorf I, Zirlik A, Hedrick CC, Ley K, Wolf D. Atlas of the Immune Cell Repertoire in Mouse Atherosclerosis Defined by Single-Cell RNASequencing and Mass Cytometry. Circ Res 2018;122:1675-1688.

8. Dobnikar L, Taylor AL, Chappell J, Oldach P, Harman JL, Oerton E, Dzierzak E, Bennett MR, Spivakov M, Jorgensen HF. Disease-relevant transcriptional signatures identified in individual smooth muscle cells from healthy mouse vessels. Nat Commun 2018;9:4567.

9. Fernandez DM, Rahman AH, Fernandez NF, Chudnovskiy A, Amir ED, Amadori L, Khan NS, Wong CK, Shamailova R, Hill CA, Wang Z, Remark R, Li JR, Pina C, Faries C, Awad AJ, Moss N, Bjorkegren JLM, Kim-Schulze S, Gnjatic S, Ma'ayan A, Mocco J, Faries $P$, Merad M, Giannarelli C. Single-cell immune landscape of human atherosclerotic plaques. Nat Med 2019;25:1576-1588. 
Impact | 10 

Appendices

List of abbreviations 



\begin{tabular}{|c|c|}
\hline AAlx & Ankle-arm index \\
\hline ACTA2 & Alpha-actin 2 \\
\hline ADAM17 & A disintegrin and metalloprotease 17 \\
\hline ApoE & Apolipoprotein E \\
\hline Bax & Bcl-2 associated $x$ \\
\hline $\mathrm{Bcl}-2$ & B-cell lymphoma 2 \\
\hline BiKE & Biobank of Karolinska endarterectomies \\
\hline BMDM & Bone marrow derived macrophages \\
\hline BNIP3 & $\mathrm{Bcl}-2$ nineteen kilodalton interacting protein \\
\hline CAIX & Carbonic anhydrase IX \\
\hline CEA & Carotid endarterectomy \\
\hline cIMT & Intima-media thickness of the carotid artery \\
\hline CKD & Chronic kidney disease \\
\hline c-Kit & Stem cell factor receptor \\
\hline cKO & Conditional knock-out \\
\hline Col & Collagen \\
\hline CMA & Chaperone mediated autophagy \\
\hline CODAM & Cohort on diabetes and atherosclerosis Maastricht \\
\hline $\operatorname{cox} 2$ & Cytochrome c oxidase 2 \\
\hline CVD & Cardiovascular disease \\
\hline CVE & Cardiovascular event \\
\hline DAMP & Damage associated molecule pattern \\
\hline DEG & Differentially expressed gene \\
\hline dKO & Double knock-out \\
\hline Dpep1 & Di-peptidase 1 \\
\hline EC & Endothelial cell \\
\hline ECM & Extracellular matrix \\
\hline EndMT & Endothelial-to-mesenchymal transition \\
\hline
\end{tabular}




\section{Appendices | List of abbreviations \\ eNOS Endothelial nitric oxide synthase \\ EMT Epitelial mesenchymal transition \\ EPO Erythropoietin}

FACS Fluorescence assisted cell sorting

Fbln1 Fibulin 1

FAP Fibroblast activation protein

FFPE Formalin-fixed paraffin embedded

FSP-1 Fibroblast-specific protein 1

Gli-1 GLI family zinc finger 1

GLUT1 Glucose transporter 1

GO Gene ontology

HIF Hypoxia inducible factor

HMBG1 High mobility group box protein-1

HRE Hypoxia responsive element

HSC70 Heat shock cognate protein 70

ICAM1 Intercellular adhesion molecule 1

IFN $\quad$ Interferon gamma

IL Interleukin

iNOS inducible nitric oxide synthase

IPA Ingenuity pathway analysis

$\mathrm{IPH} \quad$ Intraplaque hemorrhage

IT Intimal thickening

$\mathrm{KI} \quad$ Knock-in

KLF Krüppel like factor

KO Knock-out

LAMP1 Lysosome-associated membrane protein 1

LAMP2A Lysosome-associated membrane protein type 2A

LDL Low density lipoprotein 
LDLR Low density lipoprotein receptor

LGALS3 Galectin-3

Lifr Leukemia inhibitory factor receptor

LPS Lipopolysaccharide

Lum Lumican

MaasHPS Maastricht human plaque study

MMP Matrix metalloproteinase

MPTC Maastricht pathology tissue collection

MSC Mesenchymal stem cell

MYH11 Myosin heavy chain 11

NFkB Nuclear factor kappa-light-chain-enhancer of activated B cells

NO Nitric oxide

oxLDL oxidized low density lipoprotein

p21 cyclin-dependent kinase inhibitor 1a

p27 cyclin-dependent kinase inhibitor $1 b$

p53 tumor protein 53

PAI-1 Plasminogen activator inhibitor type 1

PCA Principle component analysis

PCSK9 Proprotein convertase subtilisin/kexin type 9

PDGFB Platelet derived growth factor B

Pgdfra Platelet derived growth factor receptor alpha

Pdgfr $\beta \quad$ Platelet derived growth factor receptor beta

PHD Prolyl hydroxylase domain protein

PIT Pathological intimal thickening

PVHL $\quad$ von Hippel-Lindau protiein

qPCR Quantitative polymerase chain reaction

RAR Retinoid acid receptor

RER Respiratory exchange ratio 


\begin{tabular}{|c|c|}
\hline RNAseq & RNA sequencing \\
\hline ROS & Reactive oxygen species \\
\hline sCAIX & Soluble carbonic anhydrase IX \\
\hline Sca-1 & Stem cell antigen 1 \\
\hline SCS & Single cell sequencing \\
\hline sFRP1 & Secreted frizzled related protein 1 \\
\hline SMC & Smooth muscle cell \\
\hline Smoc2 & Sparc related modular calcium binding 2 \\
\hline Spp1 & Osteopontin \\
\hline Tcf-21 & T cell factor 21 \\
\hline TG & Triglycerides \\
\hline TGF $\beta$ & Transforming growth factor beta \\
\hline $\operatorname{Tg} \ln$ & Transgelin \\
\hline TkFCA & Thick fibrous cap atheroma \\
\hline $\mathrm{TNF} \alpha$ & Tumor necrosis factor alpha \\
\hline TREM2 & Triggering receptor expressed on myeloid cells 2 \\
\hline tSNE & T-stochastic neighboring embedding algorithm \\
\hline UMI & Unique molecular identifier \\
\hline UMAP & Uniform manifold approximation and projection \\
\hline UPR & Unfolded protein response \\
\hline VCAM1 & Vascular cell adhesion molecule 1 \\
\hline VEGF & Vascular endothelial growth factor \\
\hline VLDL & Very low density lipoprotein \\
\hline WD & Western diet \\
\hline WT & Wildtype \\
\hline aSMA & alpha smooth muscle actin \\
\hline
\end{tabular}


List of abbreviations | Appendices 

Appendices

Acknowledgements - Dankwoord 

Wauw wat zijn deze 4 jaar voorbij gevlogen en wat was het een avontuur, zowel op academisch als sociaal vlak. Hier wil ik alle mensen die hebben bijgedragen aan mijn avontuur bedanken.

Prof. Dr. Sluimer, Judith, dit hoofdstuk begint natuurlijk bij jou. Toen ik 4 jaar geleden bij je binnen kwam voor een sollicitatiegesprek, wist ik het meteen: dit is een match! Toentertijd werd ik niet aangenomen voor de baan waarvoor ik op gesprek kwam, maar gelukkig kwam er de Dekker beurs en mocht ik alsnog beginnen. De afgelopen 4 jaar heb ik ontzettend veel van je geleerd en motiveerde jij me altijd om het onderste uit de academische kan te halen. Ik ben trots dat je je last-minute alsnog mijn promotor mocht noemen. Ons team heeft al vele namen gehad (Team eggplant, Teamplayers en Team 2020) en ik heb het geluk dat ik nog even van dit team deel uit mag maken. Ik wil je bedanken voor je vertrouwen, de vele koffies, wijntjes, congressen met te gekke feestjes en academische discussies. Als kers op de taart, mochten de teamplayers naar jouw sprookjesbruiloft met Andy komen. Wat een geweldige dag was dat! Kort samengevat (zoals jij dat altijd zo fijn doet): ik had me geen betere supervisor kunnen wensen!

Prof. Dr. Biessen, Erik, dankjewel voor alle discussies die we hadden in onze maandelijkse meetings. Als ik even vast liep met een van mijn projecten, kwam jij vaak met een nieuw puntje waar we nog aan konden denken. Ik blijf nog even bij Pathologie, maar ik hoop dat ik op een dag zo'n super speech mag ontvangen van je!

Prof. Dr. Baker, Andy, thank you for the guidance the last years. Your fresh look on things and reassuring Judith and me that we had plenty of data, always gave me confidence about the data. Many thanks for all your input!

I would like to thank all the members of my assessment committee Prof. Kooi, Prof. Post, Prof. Zernecke, Dr. LaPointe and Dr. Jørgensen for investing their time in reading and assessing my thesis.

Prof. dr. Axel zur Hausen, bedankt dat ik deze 4 jaar bij pathologie heb mogen werken.

Margaux en Jan, mijn paranimfen. Bijna heel mijn PhD avontuur waren jullie aan mijn zijde, wat hebben we't leuk gehad!! Margaux, ma Gougou, what a ride it has been. Van collega's naar vriendinnen naar roomies. Zonder jou was deze PhD een stuk saaier geweest, al die feestjes, congressen (Goat smell in) en ons grootste avontuur: Chicago! Wauw, wat was dat cool. Als het even niet zo ging als we wilden op het lab, gingen we lekker koffie drinken (of alcohol). We steunden elkaar waar nodig en daar heb ik ontzettend veel aan gehad. Lieve Gougou, in jou heb ik een vriendin voor het leven gevonden. Ma copine, merci pour tout, les bonnes et les mauvais moments. Jan, Jannieboy, danke für die letste 4 jahre. We started our PhD almost at the same time and we're still here together. We had so many fun parties and boy, we laughed and drank a lot.. Even though we're currently still discussing whether 


\section{Appendices | Acknowledgements - Dankwoord}

you can freeze bread, I sincerely hope you will be able to answer this question when you get your PhD!! I'm proud of you and us, of what we have accomplished in the last 4 years! Thank you both so, so much!

Lauren, I met you very early in my PhD and soon after that, we formed the triangle with Margaux (also known as Team Triangle or KiLaMa). Thank you for always being there for me and always making it possible to have a glass of wine, which is of course needed at all times. Together with Margaux, we had so many nights at VBML and Basilica; I loved every second of it!! I hope that the KiLaMa keeps on riding for a long time, which now only consists of doctors. Look at us!!

Jasper en Jenny, team eggplant begon met jullie twee erbij. Jasper, we hebben heel wat afgereisd samen: Amsterdam, Biezenmortel, Helsinki, Boston, Maine. Het was een eer om bij jouw verdediging paranimf te mogen zijn en wat deed je het goed! Tegenwoordig zit je ver weg van het zonnige zuiden en ben je terug in je doktersrol. Gelukkig kon ik nog steeds op je rekenen voor advies over de twee hoofdstukken waar we samen aan hebben gewerkt. Dankjewel voor je collegialiteit, leuke feestjes en fijne gesprekken. Jenny, ik weet niet of ik ooit iemand heb ontmoet die zo'n groot zorghart heeft als jij. Altijd stond je voor iedereen klaar, binnen en buiten de pathologie afdeling. Dankjewel daarvoor! Wat hebben we mogen genieten van al je bak- en kookkunsten (ik mis die banoffee pie nog steeds). Ik ben trots op je hoe je je carrière keuzes hebt gemaakt en ik weet $100 \%$ zeker dat je helemaal op je plekje bent tussen de oudjes, dokter Jenny.

Renée, jij bent begonnen als mijn stagiaire en bent gelukkig gebleven in ons team als $\mathrm{PhD}$ student. Jij weet als geen ander dat een PhD gelijk staat aan vallen en opstaan en het mag zeker benoemd worden dat jij je daar, soms met wat achtergrond stress, super doorheen slaat. Je bent een echte aanwinst voor ons team en ik ben blij dat we nog even samen mogen werken! Dankjewel voor de leuke discussies en heel veel succes met jouw PhD, dat gaat natuurlijk helemaal goed komen! Sebastiaan, de nieuwste aanwinst van ons team. Hopelijk ben je op je plek hier in het zonnige zuiden van Nederland. Heel veel succes met je PhD! Olivia, Han, Chang, Taghi, Ruud, Adele, Anke, Daniëlle, Valeria, Laura and Elias thank you all for your scientific and non-scientific conversations. Good luck to you all in finishing your $\mathrm{PhD}$ ! Thomas \& Elke, ook al hebben we niet als échte collega's samen gewerkt, voelde dit af en toe wel zo. Jullie onderzoek staat aan de fundering van mijn thesis, dankjewel hiervoor! Hopelijk komt het tot een mooie publicatie!

De postdocs, Lieve, Suzan en Pieter, ik ben nu officieel overgestapt naar jullie kant! Lieve, dankjewel voor jouw adviezen en leuke gesprekken. De loopjes van kantoor naar koffiemachine en spontane gesprekken onderweg waren altijd ontzettend gezellig. Suzan, we hebben samen een muizenstudie opgezet en hopelijk kunnen we deze ook nog samen gaan starten! Bedankt voor alle discussies over macrofaag kweek en het falen hiervan.. 
Succes in de toekomst! Pieter, als iemand ontzettend veel feitjes weet, ben jij het wel! Ook wij hebben samen een studie mogen doen, met hopelijk een leuke publicatie als gevolg. Dankjewel voor je adviezen en succes in je academische carrière!

Marion, wat heb ik ontzettend met jou kunnen lachen, zowel op de werkvloer als op feestjes (jouw specialiteit). Ons tripje naar Edinburgh was wel een hoogtepunt, waar we allebei compleet gaar waren na een hele dag muiswerk. Bij al mijn muiswerk kon ik op je rekenen en waren we samen een goedlopend treintje. Dankjewel voor het delen van je kennis onder de microscoop en voor alle leuke gesprekken! Marjo, bedankt voor alle input tijdens labmeetings. Ik stelde het altijd zeer op prijs dat je de juiste vragen stelde, waardoor ik mijn eigen data in een nieuw licht zag.

Mat, bedankt voor je ontzettend uitgebreide lab kennis en geduld, als ik weer eens last minute met een bestelling aan kwam die de deur uit moest. Clairy, dankjewel voor de bergen coupes die je voor me hebt gesneden! Jacques, ik kon altijd op jouw rekenen als er kleuringen moesten gebeuren. Wat heb je er ontzettend veel gedaan! Ook met muiswerk, wist ik dat ik altijd bij jou kon aankloppen. Bedankt hiervoor! Gregorio, Erwin, Jack, Dietbert \& Sylvia, dankjewel voor jullie input tijdens labmeetings of vragen die ik had over mijn data. Audrey, dankjewel dat je altijd klaar stond als er iets geregeld moest worden!

Ook wil ik alle mensen van het CPV hartelijk bedanken en in het speciaal Richard, Rik, Clarice, Nicole, Anouk en Mandy. Jullie stonden altijd voor ons klaar, zelfs als er last-minute nog muizen uitgeleverd moesten worden of we vragen hadden. Bedankt hiervoor!

Armand, Rick, Niko, en Leon, jullie staan centraal in de fijne samenwerking tussen biochemie en pathologie. Dankjewel voor al jullie input de afgelopen jaren! Collega's van de kankergroep, Nathalie, Imke \& Laura, dankjewel voor de leuke praatjes, borrels en delen van lab-frustraties over de laatste jaren.

Colleagues from abroad, thank you for all the collaborations. lan, you were the driving force behind all the bioinformatics in our fibroblast manuscript. Thank you for all your help! Francesca, thank you for the nice scientific discussions. It was a lot of fun to work with you and this resulted in a publication in Circulation Research! Monica, Christoph \& Rafael, thank you for all your input in our projects. Your help was greatly appreciated! Javier \& Julio, thank you for sharing your bioinformatic knowledge with us. I'm proud of our manuscript together!

I'mCARIM is ook een deel geweest van mijn PhD avontuur, ik wil alle leden bedanken voor hun input de laatste jaren. Ook wil de leden van de EPC bedanken voor de leuke tijd als CARIM PhD vertegenwoordigster! 


\section{Appendices | Acknowledgements - Dankwoord}

Ik wil graag ook mijn lieve vriendinnen uit Weert bedanken. Fabiënne, mijn ride or die, dankjewel dat je er altijd voor me bent. Ook al zitten we totaal niet in dezelfde sector, we snappen elkaar als het even wat minder gaat. Ik zou me geen betere vriendin kunnen wensen! Sanne, al vriendinnen sinds onze geboorte en wat ben ik blij dat we dat nog steeds zijn! Dankjewel voor je steun al die jaren! Iris \& Maartje, ook wij kennen elkaar al 10 jaar. Ik weet wat ik heb aan onze band en dat we elkaar maar hoeven te bellen en we staan bij elkaar op de stoep. Dankjewel hiervoor! Iris, wij zijn de laatste jaren hechter en hechter geworden. Als het even tegen zat, was jij er voor me. Wat ben ik blij met zo'n lieve vriendin als jij. Vera, dankjewel voor je interesse en steun over de laatste jaren. Jij weet als geen ander hoe lastig een PhD kan zijn. Bedankt voor alle fijne gesprekken. Julia, we go way back! Samen startten we onze eerste stage bij embryologie. Jij bent inmiddels doorgegroeid tot een super dokter met een leuke opleidingsplek. Bedankt voor al die momenten dat je er voor me was en we ons even konden uitlaten over ons drukke academische leven.

Pap \& mam, wat een avontuur was 't de afgelopen jaren. Als 't op t werk even mis ging, waren jullie er altijd voor mij en toonden altijd interesse. Jullie hebbe mij altijd gesteund, in alle beslissingen die ik genomen heb. Verhuizen van Wieërt naar Mestreech en terug naar Wieërt, waar Pim en ik super fijn wonen, mede dankzij jullie! Ik wil jullie vanuit de grond van mijn hart bedanken. Demi, als ik ons moest beschrijven, dan zijn we ying \& yang: compleet verschillend. Toch kan ik altijd op je rekenen, dankjewel hiervoor. Samen met Wouter, ben je nu een echt Belske. Ik wens jullie al 't geluk toe in jullie leuke huisje! Maria \& Peter, dankjewel dat jullie huis een tweede thuis voor mij mocht zijn. Steffie \& Maikel, bij jullie was het altijd gezellig en kon de druk even van de ketel af. Even spelen en knuffelen met Luca en Kyan maakte de dag altijd weer goed! Lieve Oma, wat mis ik jou.. Ik had zo graag gehad dat je bij dit moment kon zijn, ik hoop dat je trots bent!

Last, but definitely not least. Lieve Pim, dankzij jou sta ik waar ik nu sta. Ik kan altijd op je rekenen en heb je onvoorwaardelijke steun. Jij hebt me laten voelen wat echte liefde is, mijn maatje voor het leven. Ik ben trots op jou en hoe we samen in het leven staan. Op nog vele, vele jaren!

Leef minse, bedanktj vur alles!!

\section{Kim}


Acknowledgements - Dankwoord | Appendices 

Appendices

Curriculum Vitae 

Personal Information

$\begin{array}{ll}\text { Name: } & \text { Kim van Kuijk } \\ \text { Date of birth: } & 9^{\text {th }} \text { of June, } 1993 \\ \text { Place of birth: } & \text { Weert } \\ \text { Nationality: } & \text { Dutch }\end{array}$

\section{Education}

Aug. 2016 - Sept. 2020

PhD training in Biomedical Sciences

Department of Pathology, Maastricht University Medical Center

The Netherlands

Sept. 2014 - Jul. 2016

Master Biomedical Sciences (MSc)

Maastricht University

The Netherlands

Sept. 2011 - Jul. 2014

Bachelor Biomedical Sciences (BSc)

Maastricht University

The Netherlands

Research/Work experience

Sept. 2020 - current

Post doc

Department of Pathology, Maastricht University Medical Center

The Netherlands

Fibroblasts in healthy and diseased vasculature

Aug. 2016 - Sept. 2020

PhD training in Biomedical Sciences

Department of Pathology, Maastricht University Medical Center 
The Netherlands

Promotors: Dr. Judith Sluimer, Prof. Erik Biessen

Co-promotor: Prof. Andrew Baker

The effect of intra- and extracellular challenges on cellular responses in atherosclerosis

\section{MSc Senior practical training}

Department of Gynaecology \& Obstetrics, Maastricht University

The Netherlands

Supervisor: Dr. Andrea Romano

Title: Inhibition of HSD17B1 as a novel endocrine treatment in endometrial cancer.

Jan. 2015 - Jul. 2015

\section{MSc Junior practical training}

Department of Toxicology, Maastricht University

The Netherlands

Supervisor: Prof. Roger Godschalk

Title: Does chronic inflammation lead to cancer?

Apr. 2014 - Jul. 2014

BSc Practical training

Department of Anatomy \& Embryology, Maastricht University

The Netherlands

Supervisor: Dr. Ulrike von Rango

Title: Role of VEGFR1 and VEGFR2 in placental vascularization and dysfunction.

Certificates/Courses

November 2018

TMS-VRS D. Permit to work with radioactive open sources 


\section{Curriculum Vitae | Appendices}

October 2018

September 2018

October 2017

October 2017

July 2017

October 2016

January 2016

June 2015

Other activities
Hartstichting Papendal course Cardiac function \& adaptation

Scientific writing course

Teaching certificate Maastricht University

Hartstichting Papendal course Atherosclerosis \&

Thrombosis

Summer school Vasculata Chicago

Hartstichting Papendal course Vascular Biology

Art 9. Permit to work with lab animals

Microscopy certificate
Sept. $2020-$

Jun. 2020 - Current

Dec. 2016 - Jun. 2020

Sept. 2015 -Jul. 2016

Sept. 2015 - Jul. 2016

\section{Teaching}

Course 2.1 Threats \& Defences, Maastricht Univeristy

Reviewer

Cardiovascular Research, Scientific Reports

PhD representative I'mCARIM

Chair, CARIM Maastricht

Member of the Management Team Biomedical

Sciences Master

Biomedical Sciences representative, Maastricht

University

Member of the Student Council Health \& Life Sciences Master Biomedical

Sciences representative, Maastricht University

Conferences/Seminars

Keystone eSymposium

Tissue plasticity
Scientific talk

Single cell sequencing reveals fibroblast heterogeneity in healthy and diseased vasculature 
BAS meeting 2020

Online

IVBM 2020

Online

Gordon Conference 2019

Maine, USA

EAS 2019

Maastricht, Netherlands

ESM-EVBO 2019

Maastricht, Netherlands

DEBS Meeting 2018

Biezenmortel, Netherlands

IVBM 2018

Helsinki, Finland

Vasculata 2017

Chicago, USA

Papendal Hartstichting

Courses 2016-2018

DEBS Meeting 2016
Rapid fire talk

Deficiency of myeloid PHD proteins aggravates atherogenesis via macrophage apoptosis and paracrine fibrotic signaling

Poster presentation

Deficiency of myeloid PHD proteins aggravates atherogenesis via macrophage apoptosis and paracrine fibrotic signaling

Poster presentation

Single cell sequencing of healthy mouse adventitia reveals differentiation tracks of PDGFRb+cells and 6 novel, specific, mesenchymal cell markers

Selected oral presentation

Myeloid PHD2 knockdown drives macrophage apoptosis and paracrine fibroblast/smooth muscle cell collagen secretion leading to atherosclerotic plaque fibrosis

Selected oral presentation

Single cell sequencing reveals heterogeneity of adventitial mesenchymal cells in healthy and diseased mice

Poster presentation

SMC- and pericyte-independent effect of PDGFB lacking its retention motif: leukocytosis, macrophage apoptosis and MMP activity promoted murine atherosclerotic plaque size and fibrosis

Poster presentation

Myeloid knockdown of oxygen sensor prolyl hydroxylase domain protein 2 (PHD2) leads to larger, but more stable plaques in a mouse model for atherosclerosis

Poster presentation

Myeloid knockdown of oxygen sensor prolyl hydroxylase domain protein 2 (PHD2) leads to larger, but more stable plaques in a mouse model for atherosclerosis

Poster presentations

Poster presentation 


\section{Curriculum Vitae | Appendices}

Biezenmortel, Netherlands

Fibroblasts as the overlooked key player in

atherosclerosis

Awards \& Scholarships

Poster prize

IVBM 2020

DEBS meeting 2018

Selected best talk

BAS Meeting 2020

Scholarship

eKeystone Tissue plasticity 2020

\section{Publications}

Deficiency of myeloid PHD proteins aggravates atherogenesis via macrophage apoptosis and paracrine fibrotic signaling.

van Kuijk K†, Demandt JAF†, Perales-Patón J†, Theelen TL, Kuppe C, Marsch E, de Bruijn J, Jin $\mathrm{H}$, Gijbels MJ, Matic L, Mees BME, Reutelingsperger CPM, Hedin U, Biessen EAL,

Carmeliet P, Baker AH, Kramann R, Schurgers LJ, Saez-Rodriguez, Sluimer JC

+ Authors contributed equally.

In revision, Cardiovascular Research

CARMN Loss Regulates Smooth Muscle Cells and Accelerates Atherosclerosis in Mice

Francesca Vacante, Julie Rodor, Mukesh K Lalwani, Amira D Mahmoud, Matthew Bennett, Azzura De Pace, Eileen Miller, Kim van Kuijk, Jenny BG de Bruijn, Marion Gijbels, Thomas Christie Williams, Michael B Clark, Jessica P Scanlon, Amanda C Doran, Rusty Montgomery, David E Newby, Mauro Giacca, Dónal O'Carroll, Patrick WF Hadoke, Laura Denby, Judith C Sluimer, Andrew H Baker

Circ. Res. 2021 Feb 24

The hypoxia-sensor carbonic anhydrase IX affects macrophage metabolism, but is not a biomarker for human cardiovascular disease.

Demandt JAF, Dubois LJ, van Kuijk K, Zat́ovičová M, Jin H, Parkkila S, van der Laan SW, Jelenska L, Mees BME, Cleutjens KBJM, van der Kallen CJH, Schalkwijk CG, van Greevenbroek MMJ, Biessen EAL, Pasterkamp G, Pastoreková S, Stehouwer CDA, Sluimer JC

Sci Rep. 2021 Jan 11;11(1):425 


\section{Appendices | Curriculum Vitae}

Mild hyperlipidemia in mice aggravates platelet responsiveness in thrombus formation and exploration of platelet proteome and lipidome.

van Geffen JP, Swieringa F, van Kuijk K, Tullemans BME, Solari FA, Peng B, Clemetson KJ, Farndale RW, Dubois LJ, Sickmann A, Zahedi RP, Ahrends R, Biessen EAL, Heemskerk JWM,1 Sluimer JC, Kuijpers MJE

Sci Rep. 2020 Dec 8;10(1):21407

Fibroblasts in atherosclerosis: heterogeneous and plastic participants.

Tillie RJH, van Kuijk K, Sluimer JC

Current opinion in Lipidology 202001 Oct 2020, 31(5):273-278

Heterogeneity and plasticity in healthy and atherosclerotic vasculature explored by single cell sequencing.

Van Kuijk K, Kuppe C, Betsholtz C, Vanlandewijck M, Kramann R, Sluimer JC

Cardiovascular Research 2019 Oct 1;115(12):1705-1715

Blocking 17 $\beta$-hydroxysteroid dehydrogenase type 1 in endometrial cancer: a potential novel endocrine therapeutic approach.

Konings GF, Cornel KM, Xanthoulea S, Delvoux B, Skowron MA, Kooreman L, Koskimies P, Krakstad C, Salvesen HB, van Kuijk K, Schrooders YJ, Vooijs M, Groot AJ, Bongers MY, Kruitwagen RF; ENITEC, Romano A.

Journal of Pathology 2018 Feb;244(2):203-214

Atherosclerosis development: lipoproteins and beyond.

van Kuijk K, Sluimer J.C., Baker A.H.

Current opinion in lipidology 2017 Dec;28(6):520-521

Effect of interleukin (IL)-8 on benzo[a]pyrene metabolism and DNA damage in human lung epithelial cells.

Shi Q, Boots AW, Maas L, Veith C, van Kuijk K, Haenen GR, Godschalk RW, Van Schooten FJ.

Toxicology 2017 Apr 15;381:64-74 


\section{Curriculum Vitae | Appendices}

Protective role of chaperone-mediated autophagy against atherosclerosis

Madrigal-Matute J, de Bruijn J, van Kuijk K, Tiano S, Diaz A, Gijbels M, Sander B, Tasset I, Biessen EAL, Martín-Segura A, Bourdenx M, Jin H, Rensen P, Berbée J, Pasterkamp $G$, Sluimer JC, Cuervo AM

In preparation

Fibroblast heterogeneity revealed by single cell sequencing in murine healthy and atherosclerotic arteries

van Kuijk K, McCracken IR, Wichers Schreur R, Taylor RS, Dobie R, Ramachamdran P, Örd $T$, Noels $H$, Jin $H$, Wilson-Kanamori JR, Mees BME, Biessen EAL, Kaikkonen MU, Neil C. Henderson NC, Baker AH, Sluimer JC

In preparation 

